\title{
Gated Bayesian Networks
}

\author{
Marcus Bendtsen
}

UINKÖPING

Linköping University

Department of Computer and Information Science

Division for Database and Information Techniques

SE-581 85 Linköping, Sweden 
(C) Marcus Bendtsen, 2017

ISBN 978-91-7685-525-6

ISSN 0345-7524

URL http://urn.kb.se/resolve?urn=urn:nbn:se:liu:diva-136761

Published articles have been reprinted with permission from the respective copyright holder.

Typeset using $\mathrm{LAT}_{\mathrm{E}} \mathrm{X}$

Printed by LiU-Tryck, Linköping 2017 
Dedicated to my family - past, present and future - near and far. 



\title{
Gated Bayesian Networks
}

\begin{abstract}
Bayesian networks have grown to become a dominant type of model within the domain of probabilistic graphical models. Not only do they empower users with a graphical means for describing the relationships among random variables, but they also allow for (potentially) fewer parameters to estimate, and enable more efficient inference. The random variables and the relationships among them decide the structure of the directed acyclic graph that represents the Bayesian network. It is the stasis over time of these two components that we question in this thesis.

By introducing a new type of probabilistic graphical model, which we call gated Bayesian networks, we allow for the variables that we include in our model, and the relationships among them, to change over time. We introduce algorithms that can learn gated Bayesian networks that use different variables at different times, required due to the process which we are modelling going through distinct phases. We evaluate the efficacy of these algorithms within the domain of algorithmic trading, showing how the learnt gated Bayesian networks can improve upon a passive approach to trading. We also introduce algorithms that detect changes in the relationships among the random variables, allowing us to create a model that consists of several Bayesian networks, thereby revealing changes and the structure by which these changes occur. The resulting models can be used to detect the currently most appropriate Bayesian network, and we show their use in real-world examples from both the domain of sports analytics and finance.
\end{abstract}





\section{Populärvetenskaplig sammanfattning}

En grafisk modell är en beskrivning av hur olika fenomen står i relation till varandra. Ordet grafisk används för att förtydliga att denna beskrivning görs med hjälp av en graf, där fenomen är noder och relationer är bågar. Till exempel kan en grafisk modell beskriva hur olika levnadsvanor påverkar varandra, så som fysisk aktivitet, alkoholkonsumtion, matvanor och rökning. Den grafiska komponenten beskriver antaganden som leder till att beräkningar kring de fenomen som man mäter är genomförbara. Utan antaganden är det inte alltid möjligt att skapa en modell, då det kan kräva stora mängder insamlad data för att specificera modellen och beräkningarna kan ta orimligt lång tid. Felaktiga antaganden leder till att modellen presterar sämre, och idealet inträffar naturligtvis när antaganden man gjort sammanfaller med verkligheten.

Verkligheten är dock ej konstant. Över tid kan det krävas att man byter fokus till andra fenomen än tidigare, eller så ändras relationerna mellan de fenomenen som man inkluderat i sin modell. Om vi då använder en modell som inte har kapaciteten att byta fokus, eller ändra relationer över tid, så riskerar vi att få en underpresterande modell. I denna avhandling introducerar vi en ny grafisk modell som tar i beaktning det faktum att fokus och relationer förändras över tid. Vår nya grafiska modell, som vi kallar gated Bayesian networks, är en förlängning av den populära grafiska modellen Bayesian networks. Modellen kombinerar flera olika Bayesian networks och kan välja mellan dessa för att upprätthålla prestandan.

I denna avhandling introducerar vi algoritmer som tillåter oss att lära gated Bayesian networks från data. Algoritmerna varierar beroende på vad som är målet med inlärningen, samt vilka förutsättningar i form av data och kunskap man har innan själva inlärningen börjar. Till exempel visar vi hur vi kan lära oss modeller som automatiskt sköter aktiehandel på ett sådant sätt att vi minskar riskerna för förluster av det investerade kapitalet. Vi tillämpar även våra inlärningsalgoritmer för att identifiera de förändringar som sker över tid, specifikt när det gäller förändringar i volatilitet av finansiella marknader samt professionella atleters prestation. 



\section{Acknowledgments}

Writing a thesis is an amazing adventure that cannot be fully appreciated until completed. However, the people that are key to your success, and the people who make the adventure a truly enjoyable endeavour, can definitely be appreciated from the start. I have been fortunate to have two fantastic supervisors that have helped me navigate the world of research and academia. To my supervisor Jose M. Peña: I cannot thank you enough for your support, guidance, patience and humour. You have allowed me independence, and I cannot remember a meeting where we did not break out laughing at least once. You have an admirable ability of seeing things for what they are, and I can only hope that some of this clarity has rubbed off on me. To my second supervisor Nahid Shahmehri: I will always be grateful that you were willing to give me a chance to prove myself, and your support has never wavered. You have given me invaluable advice throughout my years at ADIT, and I carry with me many memorable moments from working along your side.

Throughout my years at IDA I have had the chance to work with many inspiring people, and while I wish to thank you all, I must extend a special thank you to Mariam Kamkar and Jalal Maleki. You both played a big part in me staying in academia, as you offered me a teaching position at an early stage and later facilitated my move to Ph.D. studies. You both see possibilities where others would see obstacles. I am also thankful for my colleagues at ADIT, with a special shout-out to Vengatanathan Krishnamoorthi, Zlatan Dragisic, Valentina Ivanova, and Patrick Lambrix for being loyal coffee and lunch mates. Conversing with you has often been the highlight of my day. Also a big thank you to Ulf Kargén and Niklas Carlsson for all their hard work in the courses we have given together. And then there is Dag Sonntag, my $\mathrm{Ph} . \mathrm{D}$. travelling companion and all-round good guy. I have so many wonderful memories from our travels together (often involving food), from the Christmas dinner mirage in Aalborg to incredible food in a restaurant with a somewhat dodgy entrance in Amsterdam. All I can say is that it has been a privilege that I will never forget.

I have been fortunate to not only be part of a great working environment at IDA, but also the LIIR group at IMH. It has been invaluable to work in two different research areas in parallel, exposing me to two very different research cultures. I therefore thank Ulrika Müssener, Kristin Thomas, Nadine 
Karlsson, Catharina Linderoth, Matti Leijon and Jim McCambridge. I look forward to future collaborations with you all.

I want to take this opportunity to extend a profound thank you to my family. To my extraordinary siblings Maria, Emma and Vilhelm: you are a source of rejuvenating energy, and I am looking forward to many more family activities throughout the coming years. It is also remarkable to see the next generation come to life through little Tyra. To my mother Hélène and father Preben: there are no words that can describe the support you both have given me, not only during the last few years, but throughout my life. You have cultivated in me a mindset of optimism, curiosity and gratefulness, all three of which I could never have made it this far without. You are, and have always been, my one fixed point in my otherwise sometimes impulsive lifestyle. I also wish to thank my grandparents, whose love and devotion is constantly strong, and views on life reminds me that age is just a number.

Finally, from the bottom of my heart, I thank the love of my life Evelina Johansson. You are the strongest person I know, and you achieve your goals with great determination. I adore your kindness and positive attitude. Our life together is perfect, and I promise to always be there for you, as you are always there for me.

Marcus Bendtsen

May 2017

Linköping, Sweden 




\section{Contents}

$\begin{array}{lll}1 & \text { Introduction } & 1\end{array}$

1.1 Contributions . . . . . . . . . . . . . . . . 4 4

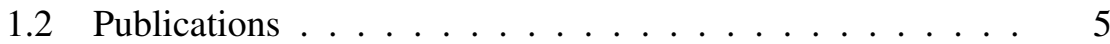

1.3 Disposition . . . . . . . . . . . . . . 6

\begin{tabular}{llll}
\hline 2 & Background & 7
\end{tabular}

$2.1 \quad$ Bayesian networks $\ldots \ldots \ldots \ldots$. . . . . . . . 7

$2.1 .1 \quad$ Reading independencies using d-separation . . . . . 9

2.1 .2 Parameter estimation . . . . . . . . . . . . . . . 11

2.1 .3 Structure learning . . . . . . . . . . . . . . . 13

2.1 .4 Inference $\ldots \ldots \ldots \ldots \ldots$

2.1 .5 Summary . . . . . . . . . . . . . . 20

2.2 Gated Bayesian networks . . . . . . . . . . . . . . . 21

2.2 .1 Structural definitions . . . . . . . . . . . . . . . 22

2.2 .2 Strategy encoding and decisions . . . . . . . . 25

2.2 .3 Execution of a gated Bayesian network . . . . . . . 26

$2.2 .4 \quad$ Execution and modelling examples . . . . . . . . 30

2.3 Related formalisms . . . . . . . . . . . . . . . . . 37

\begin{tabular}{lll}
\hline 2.3 .1 & Influence diagrams and Markov decision processes . $\quad 37$
\end{tabular}

$2.3 .2 \quad$ Hidden Markov models . . . . . . . . . . . . . . . 39

2.3 .3 Context specific independence . . . . . . . . . . . 40

2.3 .4 Other formalisms . . . . . . . . . . . . . . . . 41

2.4 Summary . . . . . . . . . . . . . . . . . 41

$3 \quad$ Learning gated Bayesian networks for algorithmic trading 43

3.1 Introduction to algorithmic trading . . . . . . . . . . . 43

3.1 .1 Evaluating alpha models . . . . . . . . . . . . . 45

3.1 .2 Benchmark . . . . . . . . . . . . . . . . . . . . . 49

3.1 .3 Technical analysis . . . . . . . . . . . . . . . 49

3.2 Template based learning . . . . . . . . . . . . . 52 
3.2.1 Gated Bayesian network template . . . . . . . . . 53

3.2 .2 Multiple simulations . . . . . . . . . . . . . 54

3.2 .3 Algorithm . . . . . . . . . . . . . . . . . . 54

3.3 Learning gated Bayesian networks for trading stocks using template based learning . . . . . . . . . . . . . 56

$3.3 .1 \quad$ Methodology . . . . . . . . . . . . . . 56

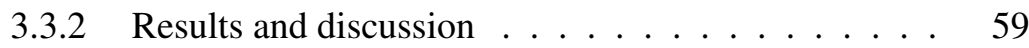

3.3.3 Extended experiments . . . . . . . . . . . . . 64

3.4 Learning using Bayesian optimisation . . . . . . . . . . 65

3.4.1 Using naïve Bayes classifiers within gated Bayesian networks for algorithmic trading . . . . . . . . . 67

3.4 .2 Gaussian processes and Bayesian optimisation . . . 68

3.4 .3 Learning algorithm . . . . . . . . . . . . 71

3.5 Learning gated Bayesian networks for index trading using Bayesian optimisation learning . . . . . . . . . . . . 71

$3.5 .1 \quad$ Methodology . . . . . . . . . . . . . . 72

3.5 .2 Results and discussion . . . . . . . . . . . . 75

3.6 Conclusions and summary $\ldots \ldots \ldots \ldots$

$\begin{array}{|lll|}4 & \text { Detecting regimes using gated Bayesian networks } & 79\end{array}$

$4.1 \quad$ Regime changes and gated Bayesian networks . . . . . . . 80

4.1 .1 Notation . . . . . . . . . . . . . . . 81

4.1 .2 Aim . . . . . . . . . . . . . . 81

4.2 Learning algorithm $\ldots \ldots \ldots$. . . . . . . . . 82

4.2 .1 Identifying regime changes in the data set . . . . . . 83

4.2 .2 Identifying regimes and structure . . . . . . . . . . 87

$4.2 .3 \quad$ Constructing a gated Bayesian network . . . . . . . 90

$4.2 .4 \quad$ Summary of learning algorithm . . . . . . . . . . 92

4.3 Related work . . . . . . . . . . . . . . . . . . . 92

4.4 Synthetic experiments . . . . . . . . . . . . . . . . . . . . . . . . . 94

$4.4 .1 \quad$ Methodology . . . . . . . . . . . . . . . . . . . . 94

4.4 .2 Results and discussion . . . . . . . . . . . . . . . . 97

4.4 .3 Conclusions . . . . . . . . . . . . . . . . . . . . 99

4.5 Regimes in baseball players' career data . . . . . . . . . . . 99

4.5 .1 The game of baseball . . . . . . . . . . . . . . 101

4.5 .2 Setup of experiments . . . . . . . . . . . . . . 105

$4.5 .3 \quad$ Methodology . . . . . . . . . . . . . . . . . . . 106

4.5 .4 Results and discussion . . . . . . . . . . . . . . . . 108

4.5 .5 Summary . . . . . . . . . . . . . . . . . 117

4.6 Volatilty regimes in financial markets $\ldots \ldots \ldots . \ldots 118$ 
$4.6 .1 \quad$ Methodology . . . . . . . . . . . . . . . . . . . 118

4.6 .2 Results and discussion . . . . . . . . . . . 120

4.7 Conclusions and summary . . . . . . . . . . . . . . 127

\begin{tabular}{|lll}
5 & Regime aware learning of Bayesian networks & 129
\end{tabular}

5.1 Regime aware learning algorithm . . . . . . . . . . . . . . . . 129

5.1 .1 Proposing hypotheses . . . . . . . . . . . . . . 131

5.1 .2 Posterior of a model . . . . . . . . . . . . . . . 131

5.1 .3 Merging subsets . . . . . . . . . . . . 133

5.2 Synthetic data experiments $\ldots \ldots \ldots \ldots . \ldots 133$

$5.2 .1 \quad$ Bayesian network structure learning and priors . . . 134

5.2 .2 Sampling . . . . . . . . . . . . . . . . 135

5.2 .3 Methodology . . . . . . . . . . . . . . . . 135

5.2 .4 Results and discussion . . . . . . . . . . . . . . 136

5.3 Revisiting volatility regimes in financial markets . . . . . 136

5.4 Summary . . . . . . . . . . . . . . . . . . . . . 139

$6 \quad$ Modelling regimes using Bayesian network mixtures $\quad 141$

6.1 Related work . . . . . . . . . . . . . . . . . . . . . 142

6.2 Model definition. . . . . . . . . . . . . . . . . . . . . . . 143

6.2 .1 Factorisation . . . . . . . . . . . . . . . 144

6.2 .2 Likelihood . . . . . . . . . . . . . . . . . 145

6.3 Parameter estimation . . . . . . . . . . . . . . . . 146

6.3 .1 Estimating new parameters . . . . . . . . . . . . . 147

6.3 .2 Computing necessary quantities . . . . . . . . . . 147

6.3 .3 Structure learning. . . . . . . . . . . . . . . . . . 149

6.4 Synthetic experiments . . . . . . . . . . . . . . . . . . . . . 149

6.4 .1 Methodology and data generation . . . . . . . . . 149

6.4 .2 Results and discussion . . . . . . . . . . . . . 150

6.5 Stock market trading revisited $\ldots \ldots \ldots \ldots$. . . . . . 151

6.5 .1 Methodology . . . . . . . . . . . . . . 151

6.5 .2 Results and discussion . . . . . . . . . . . . . . . . . 152

6.6 Summary . . . . . . . . . . . . . . . . . . . 153

7 Modelling causal scenarios using gated models 155

7.1 Context specific independence in Bayesian networks . . . . 156

7.2 Context specific independence in causal models . . . . . . . 157

7.3 Gated models . . . . . . . . . . . . . . . . . . . . 159

7.3 .1 Gates . . . . . . . . . . . . . . . . . 160

\begin{tabular}{lll}
\hline 7.3 .2 & Setting a context via conditioning or via intervention 161
\end{tabular} 
$7.3 .3 \quad$ Execution and learning . . . . . . . . . . . . . 161

7.4 Modelling causal scenarios using gated models . . . . . . . 161

7.4.1 Unstable effect and nondeterministic outcome of in-

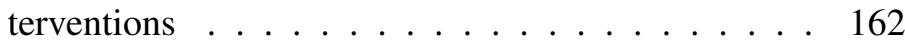

7.4.2 Mechanism dependent outcome of interventions . . 164 7.4 .3 Using gated models to identify causal effects . . . . 165

7.5 Summary . . . . . . . . . . . . . . . . 166

8 Conclusions and future work 169

$8.1 \quad$ Algorithmic trading . . . . . . . . . . . . . . . . . . . . . 169

8.2 Modelling regimes in data $\ldots \ldots \ldots \ldots$

8.3 Causal scenarios and gated models $\ldots \ldots \ldots$. . . . . . 171

\begin{tabular}{ll}
\hline Bibliography & 173
\end{tabular}

A Pseudocode for the regime detection learning algorithm 183

A.1 Identifying regime changes in the data set . . . . . . . . 183

A.2 Merging subsets . . . . . . . . . . . . . . . 185

A.3 Constructing a gated Bayesian network . . . . . . . . . 187

B Detecting regimes using hidden Markov models - experiments on synthetic data 189

B.1 Methodology . . . . . . . . . . . . . . . . . . . . . . 190

B.2 Results and discussion . . . . . . . . . . . . . . . . . 190

C Detecting regimes using hidden Markov models - experiments on synthetic baseball data 195

C.1 Methodology . . . . . . . . . . . . . . . . . 196

C.2 Results and discussion . . . . . . . . . . . . . . . . 196

D Parameter estimation and inference in Bayesian network mixtures 199

D.1 Parameter estimation . . . . . . . . . . . . . . . . . . . . . 199

D.1.1 Initial state distribution . . . . . . . . . . . . . 200

D.1.2 State transition distribution . . . . . . . . . . . . . 202

D.1.3 Observational model distribution . . . . . . . . . . . 206

D.2 Inference . . . . . . . . . . . . . . . . 211 


\section{List of Figures}

2.1 An example of a DAG that represents the independencies among a set of random variables. . . . . . . . . . . . . . . . . 8

2.2 Three constellation of nodes that play an important role in the d-separation criterion. . . . . . . . . . . . . . 10

2.3 A BN which we wish to use for inference purposes. . . . . . . . 16

\begin{tabular}{|lll|}
\hline 2.4 & In (a) the domain graph for the BN in Figure & 2.3 \\
\hline
\end{tabular} triangulated graph. In (c) a join tree constructed from the triangulated graph in (b). . . . . . . . . . . . . . . . . . . . 19

$2.5 \quad$ A junction tree primed to calculate $p\left(X_{3}\right) . \ldots \ldots \ldots$

2.6 Examples of GBNs, in (a) specific random variables are the driving forces behind transitions between the two contained models, and in (b) a utility value connected to the random variables are acting as driving forces. In (c) it is the BNs as a whole that are driving the transitions. . . . . . . . . . . 22

2.7 High-level outline of the execution algorithm. . . . . . . . . . 27

$2.8 \quad$ Pseudocode for the execution algorithm. . . . . . . . . . . . . 30

2.9 Surgery patient monitoring using a GBN. . . . . . . . . . . . 34

2.10 Modelling independence regimes using a GBN. . . . . . . . . . 36

2.11 (a) An example of an ID, (b) an example of a MDP. . . . . . . . 38

2.12 An example of a HMM. . . . . . . . . . . . . . . . . 39

3.1 Components of an algorithmic trading system. . . . . . . . . . 44

3.2 Price of an asset with buy and sell signals overlaid. . . . . . . . 45

3.3 Example of an equity curve with drawdown risks. . . . . . . . . 48

3.4 A trading screen showing the price of an asset (black) together with a MA (red). Below the price the MACD indicator (blue) and RSI (green) are plotted. . . . . . . . . . . . . . . 52

3.5 Example of a GBN template with two BN slots and two gate slots. Each BN slot has a library of pre-defined BN structures, which can be placed in each respective slot, similarly so for gates. 53 
3.6 Multiple simulations used to evaluate the performance of a learning algorithm, using a total of $n=10$ blocks and $k=3$ blocks for

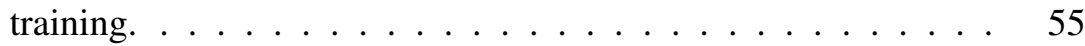

$3.7 \quad$ BNs in GBN template libraries. . . . . . . . . . . . . . . . 57

3.8 Price, signals and equity curve for IBM 2008 (left) and NVDA 2010 (right). The solid equity curve represents the GBN and the dashed equity curve represents $\mathrm{BaH} . . \ldots \ldots \ldots$

\begin{tabular}{|ll|l|l|}
\hline 3.9 & In (a), buy decisions using network 7 from Figure 3.7 & In (b), sell \\
\hline
\end{tabular} decisions using network 5 from Figure 3.7 . . . . . . . . . 63

3.10 GBN using NBCs in the different phases for buy and sell. . . . . 68

3.11 Covariance decreases by distance. . . . . . . . . . . . . . 69

3.12 GBN using NBCs in the different phases for buying and selling both long and short positions. . . . . . . . . . . . 73

$4.1 \quad$ Example proposal distributions for $\beta$ s. . . . . . . . . . . . 87

4.2 Example of merging subsets. . . . . . . . . . . . . . . 88

4.3 Example of a constructed GBN. . . . . . . . . . . . . . 90

4.4 Five regimes represented by BNs. . . . . . . . . . . . . . 95

4.5 Stylised representation of a baseball field. . . . . . . . . . . . 102

4.6 BNs created to generate synthetic career data. . . . . . . . . . . 106

4.7 Transition structures used in synthetic experiments. . . . . . . . 107

$4.8 \quad$ GBN learnt for Nyjer Morgan. . . . . . . . . . . . . . . . . . 113

4.9 Regime subsets with OPS statistic and label for Nyjer Morgan. . 113

4.10 GBN learnt for Kendrys Morales. . . . . . . . . . . . . . . . . . 114

4.11 Regime subsets with OPS statistic and label for Kendrys Morales. 114

4.12 The greatest of the three cases shown is today's true range. . . . 119

4.13 GBN learnt in the MFA experiment. . . . . . . . . . . . . 122

4.14 True range for assets in MFA with identified splits. . . . . . . . 122

4.15 GBN learnt in the ESM experiment. . . . . . . . . . . . . . 123

4.16 True range for assets in ESM with identified splits. . . . . . . . 123

5.1 Effect of constraint on hypothesis generation. . . . . . . . . 131

5.2 Stylistic view of the probabilities of the left and right subset sizes. 133

5.3 BNs in set-c. . . . . . . . . . . . . . . . . . 134

5.4 BNs in set- $v . \ldots \ldots \ldots$

5.5 Cumulative log-likelihood and splits for the MFA data set. . . . 139

5.6 Cumulative log-likelihood and splits for the ESM data set. . . . 139

6.1 Graph representation of the GBN-HMM with three time steps. . 144 


\begin{tabular}{|c|c|}
\hline 1 & In (a), a single graph that does not convey that $X \rightarrow Z$ can be \\
\hline & removed if $X>0$, a gated model representing this extra knowl- \\
\hline & edge is given in (b). When the CSI is dependent on unmodelled \\
\hline & variables, $U_{1}$ and $U_{2}$ in $(\mathrm{c})$, we cannot discern context based on \\
\hline & variables taking specific values. The gated model in (d) uses \\
\hline & threshold gates to decide which model is appropriate. . . . \\
\hline 7.2 & In (a), the effect $p(A \mid d o(T))$ is not identifiable due to confound- \\
\hline & ing between $T$ and $C$. In (b), in the context where $W=$ high, the \\
\hline & effect $p(A \mid d o(T))$ is identifiable. $\ldots \ldots \ldots \ldots$ \\
\hline$\overline{7.3}$ & Example of a gated model. . . . \\
\hline 7.4 & A single causal graph cannot capture the extra knowledge regard- \\
\hline & ing CSIs in the blood pressure example. $\ldots \ldots \ldots \ldots$ \\
\hline 7.5 & The immediate effect of low blood pressure is a move to a phys- \\
\hline & iological stable state, but may lead to transitions back and forth \\
\hline & with a crisis state. $\ldots \ldots \ldots \ldots \ldots \ldots \ldots \ldots$ \\
\hline 7.6 & The immediate effect of low blood pressure is unknown, it may \\
\hline & either lead to the stable or the crisis state. $\ldots \ldots \ldots \ldots \ldots$ \\
\hline 7.7 & In (a), the single graph does not encode the mechanism dependent \\
\hline & context, however in (b) the mechanism used to set the context \\
\hline & is part of the context itself, thus different outcomes are achived \\
\hline & depending on the mechanism used. . . . . . . . . . . . . . . \\
\hline 7.8 & A gated model using ADMGs. The causal effect $p(A \mid$ do $(T))$ \\
\hline & cannot be identified from observational data in $R_{1}$. However, ex- \\
\hline & ploiting certain CSIs the effect is identifiable in the regime model \\
\hline & $\bar{R} R_{2} . \ldots$ \\
\hline
\end{tabular}

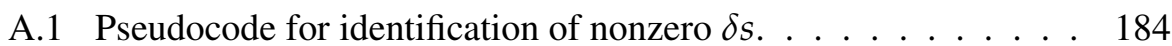

A.2 Pseudocode for regime transition structure learning. . . . . . . . 186

A.3 Pseudocode for construction of a GBN. . . . . . . . . . . . . . 188

B.1 True regimes (black line) versus the maximum probability state given a trained HMM (grey line). The $\mathrm{x}$-axis represents data points and the y-axis the regime. . . . . . . . . . . . . . . 192

B.2 True regimes (black line) versus the maximum probability state given a trained MULTI-HMM (grey line). The x-axis represents data points and the y-axis the regime. . . . . . . . . . 193

C.1 True regimes (black line) versus the maximum probability state given a trained HMM (grey line). The $\mathrm{x}$-axis represents data points and the y-axis the regime. . . . . . . . . . . . . . 197 
C.2 True regimes (black line) versus the maximum probability state given a trained MULTI-HMM (grey line). The $\mathrm{x}$-axis represents data points and the y-axis the regime. $\ldots \ldots \ldots$. . . . . . 197 


\section{List of Tables}

$1.1 \quad$ All possible assignments of four binary random variables. . . . . 2

3.1 Metric values comparing GBN with $\mathrm{BaH}$. . . . . . . . . . 61

3.2 Annual Sharpe ratio for single BN and GBN. . . . . . . . . . 62

3.3 Metric values comparing GBN, GBN with utility and BaH. . . . 66

3.4 Metric values for GBNs and $\mathrm{BaH}$ used for index trading. . . . . 75

4.1 The systems and transition structures under consideration for zero to four splits. . . . . . . . . . . . . . . . . 96

$4.2 \quad$ Actual and learnt splits using synthetic data. . . . . . . . . . . . 97

$4.3 \quad$ Accuracy of learnt GBNs on the test data sets. . . . . . . . . . . 98

4.4 Discretisation of OPS statistics. . . . . . . . . . . . . . . . . . 104

4.5 Location of identified regime transitions during GBN learning using synthetic data. . . . . . . . . . . . . . . . . 109

$4.6 \quad$ Accuracy of learnt GBNs using synthetic data. . . . . . . . . . . 109

4.7 Summary view of the GBNs learnt for a sample of baseball players. 110

4.8 Log marginal likelihood of data subsets given individual BNs (Nyjer Morgan). . . . . . . . . . . . . . . . . 116

4.9 Log marginal likelihood of data subsets given individual BNs (Kendrys Morales). . . . . . . . . . . . . . . . . 116

4.10 Data used in the MFA and ESM experiments. . . . . . . . . . . 120

4.11 Log marginal likelihood of data subsets given individual BNs

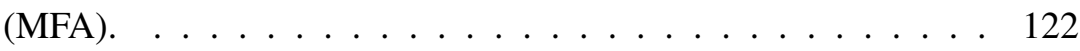

4.12 Log marginal likelihood of data subsets given individual BNs

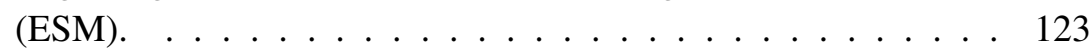

$5.1 \quad$ Results from the exp-c experiment. . . . . . . . . . . . . . . . 137

5.2 Results from the exp-v experiment. . . . . . . . . . . . . . . . 137

6.1 Means of log-likelihoods of held out data, using different predictive powers of the $Z$ variable. . . . . . . . . . . . . 151 
$6.2 \quad$ Annual Sharpe ratio comparison. . . . . . . . . . . . . . . 152 


\section{Chapter 1 Introduction}

Reasoning under uncertainty. According to Mervyn King, former governor of the Bank of England, this incredibly difficult yet everyday task has throughout history been dealt with by humans by utilising coping strategies [1]. For instance, putting away a fixed amount of money each month for retirement, which is not based on a mathematical model taking into consideration the worth of the money in the future, the probability of reaching retirement age, nor the added value the money may give today if spent. But rather, it is a heuristic which seems to work well to cope with the uncertainty of the future. Yet never has the effort been so great to take what we know, what we do not know, and what we wish to know, and create a model which allows us to reason in a formal fashion about uncertainty 1

We may think of a specific type of model as a generic template, for which we need to adjust the model's parameters for it to be useful for the reasoning that we wish to undertake. Such templates come with their own assumptions about the phenomena that we wish to model, and often the performance of the model will be linked to how close these assumptions are to the truth. In a very crude manner, we may summarise the entire field of machine learning as (in an automatic fashion) selecting among and finding the parameters of models, such that we achieve the best performance at reasoning under uncertainty.

Let us assume that we have a set of four random variables $\{X, Y, W, Z\}$. We shall assume that these random variables are binary, that is they can only take values true or false. A straightforward model to build around these variables is to attempt to create a joint distribution over them. That is, we create a table in which we write down every possible assignment to these variables, and for each assignment define a parameter which represents the probability

\footnotetext{
${ }^{1}$ King may argue against relying solely on such endeavours, since we do not know what we do not know.
} 
Table 1.1: All possible assignments of four binary random variables.

\begin{tabular}{|c|c|}
\hline Assignment & Parameter \\
\hline$X=F, Y=F, W=F, Z=F$ & $\theta_{1}$ \\
$X=F, Y=F, W=F, Z=T$ & $\theta_{2}$ \\
$X=F, Y=F, W=T, Z=F$ & $\theta_{3}$ \\
$X=F, Y=F, W=T, Z=T$ & $\theta_{4}$ \\
$X=F, Y=T, W=F, Z=F$ & $\theta_{5}$ \\
$X=F, Y=T, W=F, Z=T$ & $\theta_{6}$ \\
$X=F, Y=T, W=T, Z=F$ & $\theta_{7}$ \\
$X=F, Y=T, W=T, Z=T$ & $\theta_{8}$ \\
$X=T, Y=F, W=F, Z=F$ & $\theta_{9}$ \\
$X=T, Y=F, W=F, Z=T$ & $\theta_{10}$ \\
$X=T, Y=F, W=T, Z=F$ & $\theta_{11}$ \\
$X=T, Y=F, W=T, Z=T$ & $\theta_{12}$ \\
$X=T, Y=T, W=F, Z=F$ & $\theta_{13}$ \\
$X=T, Y=T, W=F, Z=T$ & $\theta_{14}$ \\
$X=T, Y=T, W=T, Z=F$ & $\theta_{15}$ \\
$X=T, Y=T, W=T, Z=T$ & $\theta_{16}$ \\
\hline
\end{tabular}

of the specific assignment. Such a table is offered in Table 1.1, where the $\theta$ s are the parameters of the model that we need to somehow decide upon.

To illustrate why it can be problematic estimating the 16 parameters in Table 1.1, we let the set of random variables $\{X, Y, W, Z\}$ be a representation of a patient visiting a health practitioner. Let $X$ represent if the patient has the flu, $Y$ represent if the patient has a headache, $W$ represent if the patient has a runny nose, and $Z$ represent if the patient has a fever. In order to complete Table 1.1 we must ask the health practitioner for the probabilities of each case, e.g. for $\theta_{6}$ we must ask "what is the probability that a patient does not have the flu, does have a headache, does not have a runny nose, and does have a fever", and furthermore we must also ask the practitioner to separate this case from the one where "the patient does not have the flu, does not have a headache, does have a runny nose, and does have a fever" which $\theta_{4}$ represents. It is clear that most health practitioners would struggle giving good estimates in this task. To circumvent this, we might decide to sit at a clinic and interview patients as they come and go, thereby counting the occurrences of each case. If we sat long enough we might interview enough patients to get good estimates to these 16 parameters.

Some practitioners might object, saying that they are indeed capable of estimating these 16 parameters. However if we up the ante somewhat, adjusting our initial assumption that $X, Y, W$ and $Z$ were all binary, and now assume that they take a value on a five point scale, then we end up with $5^{4}=625$ parameters in our table. Still, given enough data we could estimate the parameters from observations that we have made, however in gen- 
eral modelling the joint distribution directly is not feasible. For instance, in one of our applications in this thesis we would end up with more than 160 000 parameters.

\section{Conditional independence}

To deal with the issue of an increasing number of parameters we incorporate the concept of conditional independence. As before, let $\{X, Y, W, Z\}$ be a set of random variables (the variables may be a representation of a patient, indicators of an economy, atmospheric properties, etc.). Assume now that we were told that if you know the value of $W$, then the probability of $Y$ taking any value is not a function of the value of $X$. We say that $Y$ is conditionally independent of $X$ given $W$, and use the notation $Y \Perp X \mid W$ to express this. We may extend this notation to $Y \Perp X, Z \mid W$ which would mean that $Y$ is independent of both $X$ and $Z$ given that we know the value of $W$. In terms of probability, conditional independence implies that $p(Y \mid W, X, Z)=p(Y \mid W)$ if and only if $Y \Perp X, Z \mid W$.

The implication of the additional knowledge $Y \Perp X, Z \mid W$ on the number of parameters in Table 1.1 is a reduction from 16 parameters to 12. This is because we are now allowed to have one table for $Y$ and $W$ (which would have 4 parameters) and one table for $X, Z, W$ (which would have 8 parameters). While this reduction may not seem very significant in this case, consider again changing the variables from binary to having five states: the reduction is now from 625 parameters to 150 .

\section{Bayesian networks and gated Bayesian networks}

Introduced by Judea Pearl in 1988 [2], Bayesian networks (BNs) are carriers of independence statements and tables with parameters. They are part of a family of models which we call probabilistic graphical models. We shall have more to say about BNs in Chapter2, however it is convenient to think of them now as models which in a graphical manner convey the independencies among the random variables that we wish to model, while at the same time giving us the tables that we need to fill in. Today we have at our disposal algorithms that can learn BNs from data that we have collected, in such a way that we are given both the independencies and the tables pre-filled.

Through the publications upon which this thesis is written, we have proposed and developed a new member of the probabilistic graphical model family. We call this new model a gated Bayesian network (GBN), and it consists of several BNs connected using so called gates. From a high-level view, we 
can say that GBNs allow us to do two things: first, we can have BNs over different random variables, such that we can switch our focus from one set of random variables to another, and secondly we can have BNs over the same random variables but instead switch between different sets of independence statements. It is the gates that connect the BNs which define criteria that decide when we should switch between the different BNs. A typical use-case for the GBN is that of a trader wanting to buy and sell shares of some company. While the trader does not own any shares, he or she will use a set of random variables to reason about the future value of the company. At some point in time an opportune time reveals itself, and the trader buys shares in the company. The trader then switches focus to another set of random variables that are used to reason about potential downturn in the economy, which may affect the revenue of the company, and if there is a substantial risk of such downturn then the trader sells the shares and goes back to focusing on looking for opportunities to buy shares again. The GBN model can be built and tuned to maximise certain outcomes in this scenario, for instance to increase the reward that the trader may reap from buying and selling shares.

\subsection{Contributions}

This thesis is a treatment of a new probabilistic graphical model, and therefore our contributions are focused on the definition, development and evaluation of this new model. We have developed a set of structural definitions that explain how GBNs can be constructed, and have defined an algorithm which can be used to execute the GBN as data is collected and entered into the model. We have identified two major areas where GBNs can be used. First, we have used them to represent a process within which decisions are made, and with this goal in mind we have developed algorithms which can learn GBNs that aim to improve the outcomes of these decisions. Second, we have used them to identify and detect regime changes of some system under observation. For this task we have developed an algorithm which can learn GBNs that represent the structure of these regime changes, while also identifying the appropriate model for each regime. With respect to using GBNs for modelling a decision process, we have shown how we can learn GBNs that actively trade financial assets in such a way that they improve upon a common passive strategy. We have used our regime identification algorithm to show how the model can be used as a tool for coaches and managers of baseball teams to identify changes in players' performance. We have also used the regime identification algorithm to identify volatility regime changes 
in financial markets, specifically showing how changes developed over the most recent global financial crisis.

Apart from our contribution to the development of GBNs, we have also explored other models with similar use cases. We have developed an algorithm that attempts to, in an online fashion, identify the most appropriate BN while we collect data over time, so as to adapt to changes resembling concept drift in the data. We have also extended the popular hidden Markov model with features from the GBN. Finally, we have explored the possibility of using GBNs in a causal inference context, using them to identify regimes within which more causal effects may be identified than would be possible if regimes were ignored.

\subsection{Publications}

This thesis is based on the following publications.

- M. Bendtsen, "Regimes in baseball players' career data," Data Mining and Knowledge Discovery, 2017, accepted.

- M. Bendtsen and J. M. Peña, "Modelling regimes with Bayesian network mixtures," in Proceedings of the Thirtieth Annual Workshop of the Swedish Artificial Intelligence Society, pp. 20-29, 2017.

- J. M. Peña and M. Bendtsen, "Causal effect identification in acyclic directed mixed graphs and gated models," International Journal of Approximate Reasoning, 2017, accepted.

- M. Bendtsen, "Regime aware learning," in Proceedings of the Eighth International Conference on Probabilistic Graphical Models, pp. 1-12, 2016.

- M. Bendtsen and J. M. Peña, "Gated Bayesian networks for algorithmic trading," International Journal of Approximate Reasoning, vol. 69, no. 1, pp. 58-80, 2016.

- M. Bendtsen, "Bayesian optimisation of gated Bayesian networks for algorithmic trading," in Proceedings of the Twelfth Annual Bayesian Modeling Applications Workshop, pp. 2-11, 2015.

- M. Bendtsen and J. M. Peña, "Learning gated Bayesian networks for algorithmic trading," in Proceedings of the Seventh European Workshop on Probabilistic Graphical Models, pp. 49-64, 2014. 
- M. Bendtsen and J. M. Peña, "Gated Bayesian networks," in Proceedings of the Twelfth Scandinavian Conference on Artificial Intelligence, pp. 35-44, 2013.

\subsection{Disposition}

The rest of this thesis is structured as follows. In Chapter 2 we shall begin by giving a brief introduction to BNs, followed by a set of structural definitions that describe how GBNs can be built. We shall also define an algorithm which can be used to execute a GBN, and offer a few examples of GBNs and their execution. We shall end the chapter with a discussion of related formalisms.

In Chapter 3 we will introduce two different algorithms that can be used to learn GBNs to be used to improve upon some outcome metric within a decision making process. We shall specifically use GBNs as part of an algorithmic trading system, and see how our learnt GBNs improve upon a passive strategy. In Chapter 4 we turn our attention to regime changes. We shall propose a learning algorithm that identifies regime changes, but also creates a full GBN that represents the structure of these regime changes, and that can detect changes as data is entered to the model. We shall show how the learnt algorithms perform on both synthetic and real-world data, specifically on baseball players' career data and data from financial markets.

In Chapter 5 we shall put aside GBNs and offer an online algorithm that can be used to learn a sequence of BNs, where the last $\mathrm{BN}$ in the sequence is the one that represents the current regime of the system under observation. We shall see how the algorithm learns sequences that adapt to changes in the data that resembles concept drift. We shall specifically show how volatility regimes in financial markets can be captured. In Chapter 6 we shall extend the popular hidden Markov model with two features that are inspired by our GBN model. The extension is tested on both synthetic and real-world data, showing how the already popular hidden Markov model can be improved upon.

In Chapter7 7 we explore another potential use of GBNs within the domain of causal inference. Here we use GBNs as a language that exposes regimes in such a way that it may be possible to identify more causal effects than would be possible if regimes were ignored. We shall also offer a few examples of how, within a similar vein, GBNs can represent certain phenomena that may arise when interventions are put in place, such as unstable effects or nondeterministic outcomes. Finally, we shall offer a summary of this thesis, and our conclusions, in Chapter 8 . 


\section{Chapter 2 \\ Background}

In this chapter we shall begin with an introduction to BNs in Section 2.1 including central concepts such as d-separation, parameter estimation, structure learning and inference. Thereafter we will introduce and define GBNs in Section 2.2, including their building blocks, how they are executed and several walk-through examples. We will defer the topic of learning GBNs to Chapters 3 and 4 . Before ending this chapter, we shall in Section 2.3 offer an overview of other formalisms that are related to the proposed GBN.

\subsection{Bayesian networks}

We shall in this section offer a high-level introduction to BNs, adapted and synthesised from several excellent sources. For a full treatment we recommend all of these original publications [2, 11, 12, 13].

Given a set of random variables $\mathbf{X}$, a $\mathrm{BN}$ over these variables consists of two components. First, a description of the independencies among $\mathbf{X}$, i.e. statements that express under which conditions variables do not influence each other. This description can be conveniently conveyed using a directed acyclic graph (DAG). The second component of BNs is a factorisation of the full joint distribution over $\mathbf{X}$ which, by utilising the independencies conveyed by the DAG, can be expressed using smaller marginal and conditional distributions.

In Figure 2.1 a DAG is depicted with four nodes and three edges. The nodes $\{W, Y, Q, Z\}$ represent random variables, while edges represent potential direct association between two variables. The absence of an edge, for instance between $W$ and $Q$, represents that $W$ and $Q$ do not directly influence each other, however they may still influence each other via other variables. $Y$ is called a parent of $W$ since there is a edge from $Y$ to $W$, likewise $Z$ is a parent of $Y$, etc. A directed path is a sequence of nodes which follow the 


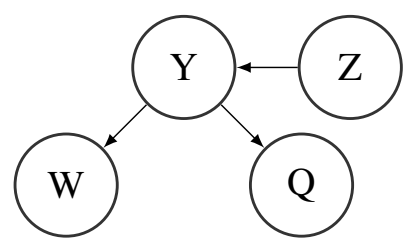

Figure 2.1: An example of a DAG that represents the independencies among a set of random variables.

direction of the edges, e.g. $Z, Y, W$ is a directed path, while $W, Y, Q$ is a path (but not directed). A node's descendants are the nodes to which a directed path can be created from the node itself, e.g. $W$ is a descendant of $Z$. As the name implies, a node's non-descendants are all nodes that are not descendants, e.g. $Y, Z$ and $Q$ are all non-descendants of $W$.

As mentioned, the DAG represents independencies among the random variables, and crucially it communicates that: if we know the values of the parents of a variable, then the variable is independent of all its other nondescendants. For instance, if we were told the value of $Y$, then our belief about the value of $W$ would be independent of the value of $Q$ and $Z$. However, if we did not know the value of $Y$, then our belief about the value of $W$ would change if we changed the values of $Z$ and $Q$.

These independencies lead us to the second component of BNs, an (potentially) economical factorisation of the joint distribution over the random variables. Consider the same example with random variables $\{W, Y, Q, Z\}$. We know by the chain rule of probability that we can break down the joint distribution $p(W, Y, Q, Z)$ into conditional distributions, and that the order in which we do so does not matter, thus we can factorise the joint distribution in several ways, as in Equation 2.1 .

$$
\begin{aligned}
p(W, Y, Q, Z) & =p(W \mid Y, Q, Z) p(Y \mid Q, Z) p(Q \mid Z) p(Z) \\
& =p(Z \mid Q, Y, W) p(Q \mid Y, W) p(Y \mid W) p(W) \\
& =p(W \mid Q, Y, Z) p(Q \mid Y, Z) p(Y \mid Z) p(Z) \\
& =\ldots
\end{aligned}
$$

All of the factorisations in Equation 2.1 are equivalent, however if we decided to look at the third factorisation in detail, we can see that we can use the independencies communicated via the DAG to reduce some of the factors. The DAG communicates that $W$ is independent of $Q$ and $Z$ if we know $Y$, thus we can reduce $p(W \mid Q, Y, Z)$ to $p(W \mid Y)$, likewise $p(Q \mid Y, Z)$ can be reduced to $p(Q \mid Y)$. The final factorisation is therefore 
$p(W \mid Y) p(Q \mid Y) p(Y \mid Z) p(Z)$. As we can see, we have successfully reduced the full joint distributions into a factorisation containing conditional distributions involving only a random variable and its parents (and marginal distributions in the case of parentless nodes). Given a set of random variables $\mathbf{X}$, where $\Pi\left(X_{j}\right)$ represents the parents of random variable $X_{j} \in \mathbf{X}$, we can define the chain rule for Bayesian networks by Equation 2.2 [12, 13]:

$$
p(\mathbf{X})=\prod_{X_{j} \in \mathbf{X}} p\left(X_{j} \mid \Pi\left(X_{j}\right)\right)
$$

Thus, the DAG not only communicates independencies among the random variables, but also immediately tells us which conditional (and marginal) distributions that we must estimate in order to define a full joint distribution. This naturally requires that the independencies that the DAG communicates actually do exist, i.e. that the joint distribution over the random variables actually contains these independencies. If it is the case that a joint distribution $p(\mathbf{X})$ contains the independencies defined via a DAG, and therefore admits the factorisation defined by the DAG, then we say that they are Markov relative.

The conditional independence relationship satisfies several properties which we can use to extract more independence statements. It is for instance decomposable, i.e. if $Q \Perp W, Z \mid Y$ then $Q \Perp Z \mid Y$, and it is symmetric, so if $Q \Perp Z \mid Y$ then $Z \Perp Q \mid Y$. Looking at the DAG in Figure 2.1 we know from before that $Q \Perp W, Z \mid Y$ (i.e. $Q$ is independent of all its non-descendants given its parent $Y$ ), but using the properties of conditional independence we also know that this must imply that $Z \Perp Q \mid Y$. It turns out that using a graphical criterion, which is known as d-separation. 1] we can read off every independence that a DAG entails, and these independencies hold in any distribution that is Markov relative to the DAG.

\subsubsection{Reading independencies using $d$-separation}

Let $\mathbf{X}, \mathbf{Y}$ and $\mathbf{Z}$ be three disjoint sets of random variables. A path between a node $X_{i} \in \mathbf{X}$ and $Y_{j} \in \mathbf{Y}$ is blocked by $\mathbf{Z}$ if and only if either of the following is true:

- Along the path there exists a chain of nodes $A \rightarrow B \rightarrow C$ or a fork $A \leftarrow B \rightarrow C$, and $B$ is in $\mathbf{Z}$.

\footnotetext{
${ }^{1}$ The $\mathrm{d}$ in d-seperation stands for directional [11].
} 


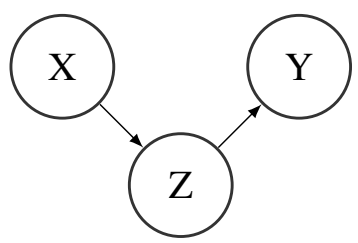

(a)

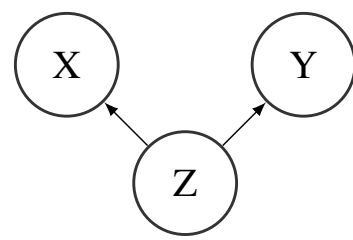

(b)

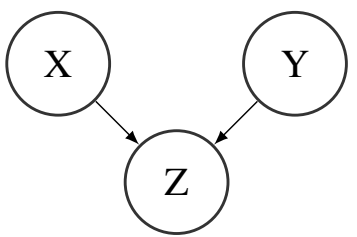

(c)

Figure 2.2: Three constellation of nodes that play an important role in the d-separation criterion.

- Along the path there exists a collider $A \rightarrow B \leftarrow C$, and $B$ is not in $\mathbf{Z}$ and neither is any of $B$ 's descendants.

If $\mathbf{Z}$ blocks every path between a node $X_{i} \in \mathbf{X}$ and $Y_{j} \in \mathbf{Y}$, then $\mathbf{Z}$ dseparates $\mathbf{X}$ and $\mathbf{Y}$. If $\mathbf{X}$ and $\mathbf{Y}$ are $\mathbf{d}$-separated by $\mathbf{Z}$ in a DAG, then $\mathbf{X}$ is independent of $\mathbf{Y}$ given $\mathbf{Z}$ in every probability distribution that is Markov relative to the DAG [11].

The cases for when a path is considered blocked can intuitively be understood by the following examples. Consider the DAG in Figure 2.2a which contains a chain $X \rightarrow Z \rightarrow Y$, and let $X$ represent the current season, $Z$ the temperature, and $Y$ the number of ice creams sold. As long as we do not know the current temperature, then knowing whether or not it is summer may affect our belief about the number of ice creams sold. However, if we were to know the current temperature $(Z)$, then information about the current season $(X)$ would have no effect on the number of ice creams sold $(Y)$. Since this is the only path between $X$ and $Y$ we say that $Z$ d-separates $X$ from $Y$. The DAG in Figure $2.2 \mathrm{~b}$ contains a fork $X \leftarrow Z \rightarrow Y$. Now let $Z$ represent the current weather conditions (sunny or cloudy), $X$ represent the current output from a solar panel, and $Y$ the number of ice creams sold at a local beach. If we do not know if it is sunny or cloudy, i.e. we have no knowledge of $Z$, then knowing that the solar panel $(X)$ is at maximum output capacity tells us something about the number of ice creams sold at the local beach $(Y)$. However, if we already knew the weather conditions, then the information about the solar panel output would not change our belief about the number of ice creams sold.

The final case, depicted in Figure 2.2c contains a collider $X \rightarrow Z \leftarrow Y$. Let $Z$ represent whether or not a baseball field is wet, $X$ represent if it is raining, and $Y$ represent if the sprinkler system is on. Learning anything about whether or not the sprinkler system is on does not change our belief regarding whether or not it is raining, and vice versa. However, if we were 
told that the baseball field is wet, and then told that it is not raining, then we will increase our belief that the sprinkler system is on. Therefore, as long as we do not know the value of $Z$, then $X$ and $Y$ will be d-separated, however if we have information about $Z$ then the path is no longer blocked, and $X$ and $Y$ may influence each other.

A BN is a carrier of certain independence statements, all of which we can read from the DAG using d-separation. These independencies allow us to factorise a joint probability distribution according to Equation 2.2. We shall now turn our attention to how we can estimate the parameters of the resulting conditional and marginal distributions from data, giving us a complete model that we can use for inference.

\subsubsection{Parameter estimation}

In this section we shall account for how we can estimate the parameters of the conditional and marginal distributions that a $\mathrm{BN}$ defines from data. We shall only consider the case where all random variables are discrete and where the data available is complete, i.e. there is no missing data (this will suffice for illustrative purposes). The case where data is missing will be discussed in Chapter 6, and we will encounter continuous variables in Chapter 5

As before we will use a set of random variables $\{W, Y, Q, Z\}$ over which we have defined the BN depicted in Figure 2.1. We shall refer to the BN as $G$. We know that we can use Equation 2.2 to factorise the joint distribution into smaller conditional and marginal distributions (as long as the joint distribution is Markov relative to the DAG). What we are now searching for are the maximum a posteriori parameters $\theta$ given $G$ and some data $\mathcal{D}$. That is, we wish to find the $\theta$ that maximises:

$$
p(\theta \mid \mathcal{D}, G)=\frac{p(\mathcal{D} \mid \theta, G) p(\theta \mid G)}{p(\mathcal{D} \mid G)} \propto p(\mathcal{D} \mid \theta, G) p(\theta \mid G)
$$

If we use the factorisation that the $\mathrm{BN}$ defines, we can rewrite the likelihood $p(\mathcal{D} \mid \theta, G)$ to Equation 2.4. Here we let $d_{i}^{W}$ represent the value that data point $d_{i}$ assigns to random variable $W$ and $\theta_{W}$ the parameters that relate to the conditional distribution over $W$ (dividing the parameters in this fashion is licensed by the independencies described by the $\mathrm{BN}$ ).

$$
\begin{aligned}
& p(\mathcal{D} \mid \theta, G)= \\
& \prod_{d_{i} \in \mathcal{D}} p\left(d_{i}^{W} \mid d_{i}^{Y}, \theta_{W}\right) p\left(d_{i}^{Q} \mid d_{i}^{Y}, \theta_{Q}\right) p\left(d_{i}^{Y} \mid d_{i}^{Z}, \theta_{Y}\right) p\left(d_{i}^{Z} \mid \theta_{Z}\right)
\end{aligned}
$$


If we assume that the parameters of each of the conditional distributions that the $\mathrm{BN}$ defines are independent, referred to as global parameter independence [12], we can go ahead and divide the prior $p(\theta \mid G)$, giving us the posterior in Equation 2.5.

$$
\begin{aligned}
p(\theta \mid \mathcal{D}, G) \propto & p\left(\theta_{W} \mid G\right) \prod_{d_{i} \in \mathcal{D}} p\left(d_{i}^{W} \mid d_{i}^{Y}, \theta_{W}\right) \times \\
& p\left(\theta_{Q} \mid G\right) \prod_{d_{i} \in \mathcal{D}} p\left(d_{i}^{Q} \mid d_{i}^{Y}, \theta_{Q}\right) \times \\
& p\left(\theta_{Y} \mid G\right) \prod_{d_{i} \in \mathcal{D}} p\left(d_{i}^{Y} \mid d_{i}^{Z}, \theta_{Y}\right) \times \\
& p\left(\theta_{Z} \mid G\right) \prod_{d_{i} \in \mathcal{D}} p\left(d_{i}^{Z} \mid \theta_{Z}\right)
\end{aligned}
$$

It should be clear then that by finding the maximum a posteriori parameters of each of the individual conditional distributions separately, we identify the $\theta$ that maximises $p(\theta \mid \mathcal{D}, G)$.

Let us assume that $Z$ represents a coin flip: it has a probability $\psi$ of heads and $1-\psi$ of tails. We say that the coin flips follow a Bernoulli distribution with parameter $\psi$, and that the parameter $\psi$ a priori follows a beta distribution with parameters $\alpha$ and $\beta$ (assuming we want a conjugate prior). We choose $\alpha$ and $\beta$ to represent our prior belief of the distribution of $\psi$, e.g. we may say that $\alpha=1$ and $\beta=1$ so that we are assigning a prior distribution over $\psi$ such that the mean of the distribution is located at 0.5 . The posterior hyperparameters $\alpha^{\prime}$ and $\beta^{\prime}$ can then be calculated by simply adding the number of observations of heads and tails, in our case $\alpha^{\prime}=1+15$ and $\beta^{\prime}=1+35$. The posterior predictive marginal distribution over $Z$ is therefore $p(Z=$ heads $)=\int p(Z=$ heads $\mid \psi) p(\psi) d \psi=16 / 52 \approx 0.31$ and $p(Z=$ tails $)=\int p(Z=$ tails $\mid \psi) p(\psi) d \psi=36 / 52 \approx=0.69$, where the conditional over $Z$ is the Bernoulli distribution and the marginal over $\psi$ is the posterior beta distribution [13, 14]. Thus these are the $\theta_{Z}$ parameters we would use in Equation 2.5 in order to find the maximum a posteriori $\theta$.

If $Z$ has more than two states, i.e. it follows the more general categorical distribution, sometimes called the multinoulli distribution, then we can use essentially the same approach as before. We may use a Dirichlet prior where for each state $i$ we have a prior hyperparameter $\alpha_{i}$ onto which we add the number of occurrences of state $i$ to get the posterior hyperparameter $\alpha_{i}^{\prime}$. The posterior predictive marginal distribution over $Z$ will then be a categorical distribution parameterised with the posterior hyperparameters. 
What is left to consider is the case when a random variable has parents, e.g. $Q$ in our example. Parameterisation follows the same procedure as before, however we have one categorical distribution for each configuration of $Q$ 's parents. For instance, if $Y$ was a binary variable we would have two categorical distributions for $Q$, and we would update our count for the categorical distribution that corresponds to the current value of $Y$. This does however require an assumption about local independence within $p\left(\theta_{Q} \mid G\right)$, that is we assume that we can estimate the parameters for when $Y=0$ independently from when $Y=1$ [12].

So far we have seen how we can, given a DAG, factorise a joint distribution and parameterise the resulting marginal and conditional distributions. However, we have neglected to discuss how we managed to construct the DAG in the first place. In the next section we shall briefly touch upon this topic.

\subsubsection{Structure learning}

We will not attempt to give an overview of all the different structure learning techniques that have been proposed and developed for BNs, but will rather given an example from each of the two main approaches to structure learning. We will first look at constraint based learning, where we assume that we have at our disposal a method for determining if a random variable $A$ is $\mathrm{d}$-separated from a random variable $B$ given a set of random variables $\mathbf{X}$. This could be decided via a data set upon which we might run hypothesis tests. We will in particular look at the parents and children (PC) algorithm. The second approach requires us to have at our disposal a method for scoring a structure given some data. We will look at a greedy thick thinning algorithm which we shall use to increase the marginal likelihood of the data by manipulating the structure. An alternative approach that has become increasingly popular is to, from a set of independence statements, exactly find the structure that matches the independencies [15, 16].

\section{Parents and children algorithm}

Let $n b(A)$ denote the neighbours of a node $A$ in an undirected graph. Assuming that we have some technique for determining if $A \Perp B \mid \mathbf{X}$, as mentioned this may be done with hypothesis tests, then the PC algorithm works as follows [12]: 
1. Begin with a fully connected undirected graph.

2. Set $i=0$ ( $i$ will keep track of the order of the sets that we are considering as separators).

3. While there exists a node with at least $i+1$ neighbours:

- For each node A for which $|n b(A)| \geqslant i+1$ :

- For each node $B \in n b(A)$ :

- For all sets $\mathbf{X}$ such that $\mathbf{X} \subseteq n b(A) \backslash\{B\}$ and $|\mathbf{X}|=i$ :

- If $A \Perp B \mid \mathbf{X}$ then remove the link between $A$ and $B$

- Set $i=i+1$

4. For each constellation $A-C-B$ introduce the collider $A \rightarrow C \leftarrow B$ if there exists an $\mathbf{X}$, among the ones identified in step 3, such that $A \Perp B \mid \mathbf{X}$ and $C \notin \mathbf{X}$ ( $\mathbf{X}$ may be empty).

5. Apply the following rules:

a) For each constellation $A \rightarrow C-B$ introduce $A \rightarrow C \rightarrow B$.

b) For each constellation $A \rightarrow B \rightarrow C$ and $A-C$ introduce $A \rightarrow C$.

c) If rules (a) and (b) cannot be applied, then choose an undirected link and give it an arbitrary direction (avoiding the introduction of cycles or colliders with disconnected parents).

Because of rule (c), the output of the PC algorithm is not a unique DAG. However all different output DAGs will be equivalent, that is they will entail the same independencies, thus we cannot distinguish between them using data over the random variables alone. In fact, if we removed rule (c) we would end up with a graph that is not a DAG, but rather an essential graph. The essential graph represents all DAGs that are equivalent, such that if $A \rightarrow B$ is in the essential graph then all equivalent DAGs also contain this edge.

\section{Greedy thick thinning}

In real-world situations we may not have access to a mechanism to decide if $A \Perp B \mid \mathbf{X}$, or we may not have enough data to run hypothesis tests. An alternative approach to structure learning is to define a score function over different structures, and then aim to find the maximum of this function. Commonly, we care about how well our model represents the data $\mathcal{D}$ that we have 
at our disposal, which can be seen as a sample of the probability distribution that we wish to model. Therefore we wish to find the DAG $G$ with the maximum posterior probability, i.e.:

$$
p(G \mid \mathcal{D})=\frac{p(\mathcal{D} \mid G) p(G)}{p(\mathcal{D})} \propto p(\mathcal{D} \mid G) p(G)
$$

Given a DAG $G$, the term $p(\mathcal{D} \mid G)$ requires us to marginalise the parameters $\theta$ of the BN which it defines. It turns out that if we use Dirichlet priors for each of the categorical distributions within the $\mathrm{BN}$, then we can calculate Equation 2.6 in closed form. Let $\mathbf{X}=\left\{X_{1}, X_{2}, \ldots, X_{i}, \ldots, X_{n}\right\}$ be a set of random variables, and let $\# X_{i}$ represent the number of states that the variable $X_{i}$ can take and \#П $\left(X_{i}\right)$ represent the number of configurations that the parents of variable $X_{i}$ can take. Let $\alpha_{i j k}$ represent the prior hyperparameter that represents the probability of random variable $X_{i}$ taking state $k$ of the Dirichlet prior for parent configuration $j$. Also, we define $\alpha_{i j}=\sum_{k=1}^{\# X_{i}} \alpha_{i j k}$. We can then calculate $p(G \mid \mathcal{D})$ using Equation 2.7 [14], where $\Gamma$ represents the gamma function and $N_{i j k}$ represents the number of times we have seen variable $X_{i}$ take state $k$ while its parents took configuration $j$ (and $N_{i j}=\sum_{k=1}^{\# X_{i}} N_{i j k}$ ).

$$
p(G \mid \mathcal{D})=\prod_{X_{i} \in \mathbf{X}} \prod_{j=1}^{\# \Pi\left(X_{i}\right)} \frac{\Gamma\left(\alpha_{i j}\right)}{\Gamma\left(\alpha_{i j}+N_{i j}\right)} \prod_{k=1}^{\# X_{i}} \frac{\Gamma\left(\alpha_{i j k}+N_{i j k}\right)}{\Gamma\left(\alpha_{i j k}\right)}
$$

If we wish to ensure that equivalent DAGs are given the same posterior probability, then we choose $\alpha_{i j k}=\alpha \frac{1}{\# X_{i} \# \Pi\left(X_{i}\right)}$, where $\alpha$ is a user-defined value that represents the imaginary sample size (i.e. the sample size upon which the prior was decided).

If we could, we would like to exhaustively try every DAG to find the maximum of Equation 2.6. However, this is not always feasible (out of complexity concerns), and therefore a heuristic approach is often taken. We shall look at one such approach, which is known as greedy thick thinning, also described in [14]:

1. Start with an empty graph.

2. Add the directed edge that maximally increases $p(G \mid \mathcal{D})$.

3. Repeat step 2 until no addition increases $p(G \mid \mathcal{D})$.

4. Remove the directed edge that maximally increases $p(G \mid \mathcal{D})$.

5. Repeat step 4 until no removal increases $p(G \mid \mathcal{D})$. 


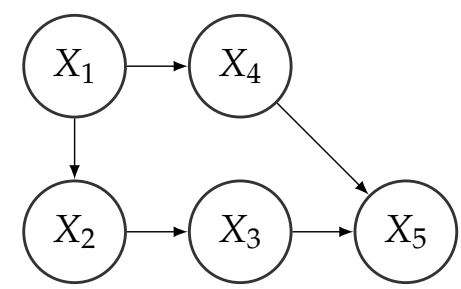

Figure 2.3: A BN which we wish to use for inference purposes.

Since in Equation 2.7 each variable is treated separately, adding or removing an edge only requires us to recompute the change in $p(G \mid \mathcal{D})$ for the resulting child variable.

\subsubsection{Inference}

So far we have only discussed how we may set up a BN, learning a structure and estimating parameters from data. Although the structure itself may be of interest, allowing us to visualise the independencies contained within a distribution, quite often we care about answering probabilistic queries. That is, we wish to compute marginal and conditional distributions given a BN. We shall therefore account for two methods for exact inference, where the outcome of our computations are not approximations. However, we note that there does exist algorithms for approximate inference, which may be necessary as the number of random variables contained in the $\mathrm{BN}$ increases, such as logic sampling, Gibbs sampling and loopy belief propagation [12, 13].

\section{Variable elimination}

Consider the BN depicted in Figure 2.3. We know that we can factorise any joint distribution that is Markov relative to the DAG according to Equation 2.2. For this example we have:

$$
\begin{aligned}
& p\left(X_{1}, X_{2}, X_{3}, X_{4}, X_{5}\right)= \\
& p\left(X_{1}\right) p\left(X_{2} \mid X_{1}\right) p\left(X_{3} \mid X_{2}\right) p\left(X_{4} \mid X_{1}\right) p\left(X_{5} \mid X_{3}, X_{4}\right)
\end{aligned}
$$

In this section we shall use potential notation, such that a distribution that operates over variables $X_{i}, \ldots, X_{j}$ is written $\phi\left(X_{i}, \ldots, X_{j}\right)$, regardless if it is a joint, marginal or conditional distribution. Thus a potential is a function that maps assignments of the variables in its domain to non-negative numbers 
(in our case they map to probabilities). Using this notation we can rewrite Equation 2.8 to Equation 2.9

$$
\begin{aligned}
& p\left(X_{1}, X_{2}, X_{3}, X_{4}, X_{5}\right)= \\
& \phi_{1}\left(X_{1}\right) \phi_{2}\left(X_{2}, X_{1}\right) \phi_{3}\left(X_{3}, X_{2}\right) \phi_{4}\left(X_{4}, X_{1}\right) \phi_{5}\left(X_{5}, X_{3}, X_{4}\right)
\end{aligned}
$$

If we wished to compute the marginal distribution $p\left(X_{3}\right)$ we could do so by multiplying all the potentials in Equation 2.9. and then sum over $X_{1}$, $X_{2}, X_{4}$ and $X_{5}$. However, the multiplication would result in an unnecessarily large number of parameters to sum over (potentially unfeasibly large). Instead, we can use the distributive property and move in the summations rather multiplying over all factors. This is known as variable elimination [12], an example of which is shown in Equation 2.10.

$$
\begin{aligned}
p\left(X_{3}\right)= & \sum_{X_{1}} \phi_{1}\left(X_{1}\right) \sum_{X_{2}} \phi_{2}\left(X_{2}, X_{1}\right) \phi_{3}\left(X_{3}, X_{2}\right) \times \\
& \sum_{X_{4}} \phi_{4}\left(X_{4}, X_{1}\right) \sum_{X_{5}} \phi_{5}\left(X_{5}, X_{3}, X_{4}\right)
\end{aligned}
$$

We begin by computing $\sum_{X_{5}} \phi_{5}\left(X_{5}, X_{3}, X_{4}\right)$. It turns out that this is equal to one, as $\phi_{5}$ is a conditional distribution over $X_{5}$ and summing all entries over $X_{5}$ necessarily adds to one. The same applies for $\sum_{X_{4}} \phi_{4}\left(X_{4}, X_{1}\right)$. We then compute $\phi_{2}^{\prime}\left(X_{1}, X_{3}\right)=\sum_{X_{2}} \phi_{2}\left(X_{2}, X_{1}\right) \phi_{3}\left(X_{3}, X_{2}\right)$, and finally we can compute the marginal $p\left(X_{3}\right)=\sum_{X_{1}} \phi_{1}\left(X_{1}\right) \phi_{2}^{\prime}\left(X_{1}, X_{3}\right)$.

These calculations are subject to the order in which we decided to marginalise the random variables, in this case the elimination order was $X_{5}$, $X_{4}, X_{2}$ and $X_{1}$. While the result of any order will be equal, i.e. the result will always be $p\left(X_{3}\right)$, the number of computations necessary may differ. For instance, consider the elimination order $X_{4}, X_{2}, X_{1}$ and $X_{5}$ shown in Equation 2.11.

$$
\begin{aligned}
p\left(X_{3}\right)= & \sum_{X_{5}} \sum_{X_{1}} \phi_{1}\left(X_{1}\right) \sum_{X_{2}} \phi_{3}\left(X_{3}, X_{2}\right) \phi_{2}\left(X_{2}, X_{1}\right) \times \\
& \sum_{X_{4}} \phi_{4}\left(X_{4}, X_{1}\right) \phi_{5}\left(X_{5}, X_{4}, X_{3}\right)
\end{aligned}
$$

Here we compute $\phi_{4}^{\prime}\left(X_{1}, X_{5}, X_{3}\right)=\sum_{X_{4}} \phi_{4}\left(X_{4}, X_{1}\right) \phi_{5}\left(X_{5}, X_{4}, X_{3}\right)$, and eliminating $X_{2}$ results in $\phi_{2}^{\prime}\left(X_{3}, X_{1}, X_{5}\right)$. Notice that we are now dealing with intermediary potentials over three random variables, while using 
the previous elimination order we only dealt with intermediary potentials over two random variables. Furthermore, we were not able to eliminate $\phi_{5}\left(X_{5}, X_{3}, X_{4}\right)$ completely in the first step. We already know that having potentials with a large domain size can be problematic (since they essentially represent tables of assignments with probabilities). Therefore, the second elimination order that we proposed could potentially require a greater number of computations than would the first (this of course depends on the number of discrete states that the variables can take).

Variable elimination has two major drawbacks: deciding which elimination order is optimal is NP-hard, which would force us to use some heuristic to decide the elimination order, and a different elimination order would have to be decided for each of the marginal distributions that we wish to compute.

To circumvent these problems to some degree, it would be beneficial if we could create a structure that would allow us to compute the marginals for all the random variables in our $\mathrm{BN}$ without having to recompute a new elimination order for each of them. Such a structure can be created using the junction tree algorithm, which we shall account for next.

\section{Junction tree algorithm}

The junction tree algorithm consists of four steps: moralising, triangulating, constructing a join tree and constructing a junction tree. We can then use message passing in the junction tree to calculate the marginals for all the random variables in our BN. In [12] the algorithm is described in much detail, here we shall offer a brief overview of the required steps.

We begin the junction tree algorithm by creating the domain graph for the $\mathrm{BN}$. The domain graph is an undirected graph which represents the domains of the potentials associated with a BN. For the BN in Figure 2.3 we have the domain graph in Figure 2.4a A link connects two nodes in the domain graph if the variables which the nodes represent are present in the domain of any of the potentials. This has the effect of moralising the graph, i.e. unconnected parents become connected (the link $X_{3}-X_{4}$ is present since they are both parents of $X_{5}$ ).

In the next step of the algorithm the graph is triangulated. A triangulated graph is one where each cycle of four or more nodes have an edge that is not part of the cycle but connects two nodes in the cycle. Triangulation can be done in several ways, and depending on which edges are added the number of computations for inference may differ (finding the optimal choice is however also NP-hard). In our running example we add one edge, resulting in the triangulated graph in Figure $2.4 \mathrm{~b}$. 


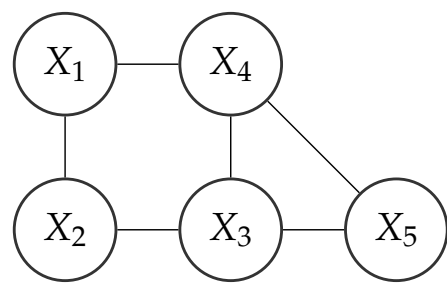

(a)

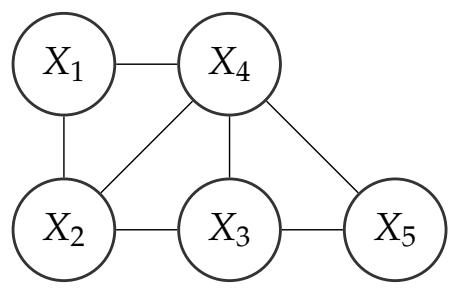

(b)

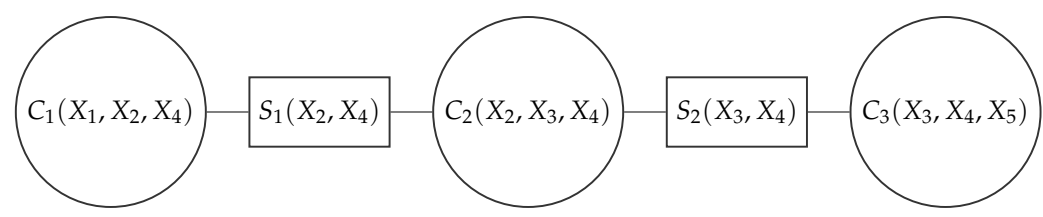

(c)

Figure 2.4: In (a) the domain graph for the $\mathrm{BN}$ in Figure 2.3, and in (b) the triangulated graph. In (c) a join tree constructed from the triangulated graph in (b).

A clique is a maximal complete set of nodes, and it can be shown that the cliques of a triangulated graph can be can be organised into a join tree [12]. A join tree is a structure such that for each pair of cliques $C_{i}$ and $C_{j}$, and each variable $X \in C_{i} \cap C_{j}$, there exists a path between $C_{i}$ and $C_{j}$ such that all nodes along the path contain $X$. The join tree will be central for the required inference propagation, and to make it explicit which information needs to be propagated between nodes, we expand the join tree with separators which contain the intersection of the two cliques that they connect. For our example we end up with the join tree in Figure $2.4 \mathrm{c}$ (note that the construction of a join tree from a triangulated graph is nondeterministic). To come to the final structure, the junction tree, we assign to each clique in the join tree a potential from the BN such that the domain of the potential is contained in the clique.

Previously our goal was to calculate $p\left(X_{3}\right)$, and in order to do so using the junction tree we identify a clique that contains $X_{3}$, and make it the root of our tree. Beginning with the leafs, we then send messages in the direction of the root, marginalising out variables not contained in the separators. This process is illustrated in the junction tree in Figure 2.5. We begin by marginalising out $X_{1}$ from the potentials held in the clique $C_{1}$, resulting in a message $\phi_{1}^{\prime}\left(X_{2}, X_{4}\right)$, and then similarly we create $\phi_{2}^{\prime}\left(X_{3}, X_{4}\right)$ by marginalising out $X_{5}$ from the potentials held in clique $C_{3}$. In clique $C_{2}$ (the root) we collect these messages, allowing us to compute $p\left(X_{3}\right)$ by marginalising out $X_{2}$ and $X_{4}$ from the potential held in $C_{2}\left(\phi_{3}\right)$ and the messages passed. 


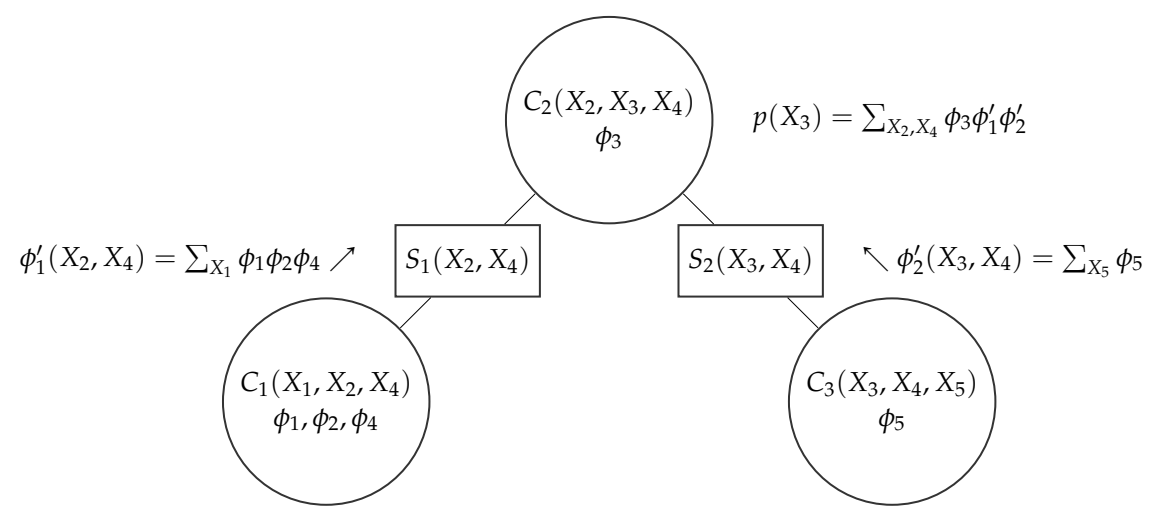

Figure 2.5: A junction tree primed to calculate $p\left(X_{3}\right)$.

If we wanted to calculate the marginals of all random variables, we can continue the message passing the other direction in the junction tree. $C_{2}$ marginalises out $X_{3}$ from the product $\phi_{3} \phi_{2}^{\prime}$ and sends it to $C_{1}$, and in a similar fashion marginalises out $X_{2}$ from the product $\phi_{3} \phi_{1}^{\prime}$ and sends it to $C_{3}$. Now that all message have been passed, calculating any marginal $p\left(X_{i}\right)$ can be done by identifying a clique which contains $X_{i}$ and marginalising out all other variables from the product of the held potentials and messages received. If we also have evidence for any of the random variables, we may introduce new 0 1 potentials in the cliques that contain the random variables (that is, potentials with all probability mass allocated according to the evidence). Once evidence potentials have been added, we can redo the sending and receiving of messages described. When evidence potentials are present we end up with a joint distribution $p\left(X_{i}, e\right)$, where $e$ represents our evidence, and we may normalise it to get the conditional over $X_{i}$, i.e. $p\left(X_{i} \mid e\right)=p\left(X_{i}, e\right) / \sum_{X_{i}} p\left(X_{i}, e\right)$.

\subsubsection{Summary}

We have shown throughout this section how a $\mathrm{BN}$ is a model that allows us to represent a full joint distribution through smaller conditional and marginal distributions. We have seen how we can learn both the structure and the parameters of the $\mathrm{BN}$ from data, and how we can use the final model for inference purposes. We shall in the next section turn our attention to GBNs, where we shall offer both structural and executional definitions. 


\subsection{Gated Bayesian networks}

Despite their popularity and advantages, there are situations where a $\mathrm{BN}$ is not enough. One such case, which we shall explore further in Chapter 3 , is when we wish to model some process that has multiple distinct phases, and for each of the phases we wish to model different random variables. The setting that we shall revisit several times in this thesis is the process of a financial asset trader, for instance buying and selling stock shares, where we want a model that can switch between identifying buying opportunities and then, once such have been found, identifying selling opportunities. The trader can be seen as being in one of two distinct phases: either looking for an opportunity to buy shares and enter the market, or an opportunity to sell shares and exit the market. These two phases can be very different and the variables included in the BNs modelling them are not necessarily the same. The second case that we shall explore in Chapters 4, 5 and 6 concerns situations where the associational relationships amongst the random variables may change over time, resembling concept drift [17], and we wish to identify these changes so that we may have multiple BNs from which one is the most appropriate at any given time. Our final case for using multiple BNs will be touched upon in Chapter7, where we use them to explain certain phenomena that manifests themselves when dealing with interventions, and as an extension allows us to identify more causal effects than would be possible using a single causal model.

Dynamic BNs (DBNs) have traditionally been used to model temporal processes using graphical models, and as their name suggests, they model the dynamics among variables between typically equally spaced time steps. However, processes that entail different models at different phases, and where the transition between phases depend on the observations made, are not easily captured by DBNs, as they assume the same static network at each time step. The need to switch between different BNs in order to model the different phases of a process was the foundation for the GBN model. We shall have reason to revisit DBNs in Chapter 4, where we will consider DBNs where the structure may change between slices (often referred to as non-stationary DBNs).

In this section we shall first introduce the building blocks of GBNs via a set of structural definitions. We shall then turn our attention to how we can make decisions based on the execution of a GBN, and exactly how a GBN is executed. Before discussing some related formalisms we shall offer a few examples that aim to highlight some of the key features of GBNs. 


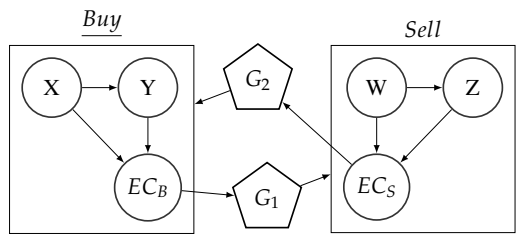

(a)

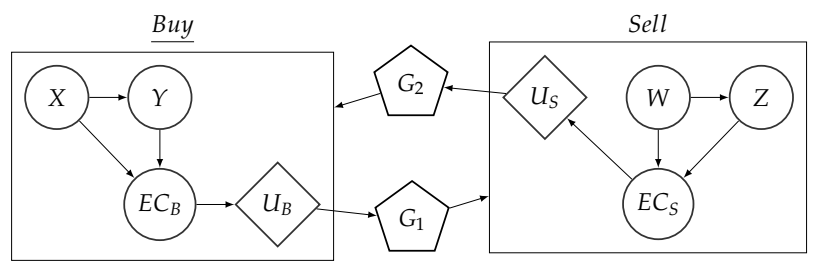

(b)

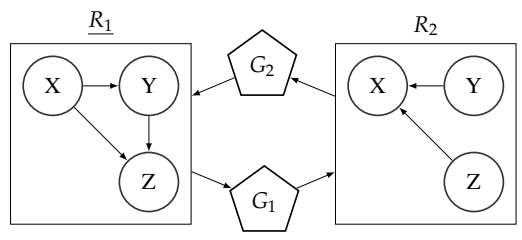

(c)

Figure 2.6: Examples of GBNs, in (a) specific random variables are the driving forces behind transitions between the two contained models, and in (b) a utility value connected to the random variables are acting as driving forces. In (c) it is the BNs as a whole that are driving the transitions.

\subsubsection{Structural definitions}

Supported by the definitions in this section, we will describe the structural semantics of GBNs and how GBNs can be used in a decision making context. In Section 2.2.3 we shall define how a GBN is executed. A GBN models a sequential process, driven by an ordered set of data points, thus it is natural to think of some index that identifies a unique position along the process. We will use $t$ to define a unique time in a temporally ordered set of data points. It is worth mentioning that the data points can be recorded at irregular times, thus the time interval between $t-1$ and $t$ can be different than $t$ and $t+1$. While reading the definitions in this section, it may be helpful to use the example GBNs offered in Figures 2.6a, 2.6b and 2.6c as reference. In Section 2.2.4 we will give three examples of GBNs that clarify and put into context the definitions of this section. 
Definition $1(\mathrm{GBN})$. A GBN consists of a set of gates $\mathcal{G}$, a set of $B N s \mathcal{B}$ and a set of directed edges $\mathcal{E}$ that connect the gates with the BNs. Let $\mathcal{B}^{A}$ be the set of active $B N s$ and $\mathcal{B}^{I}$ the set of inactive BNs. $\mathcal{B}^{A}, \mathcal{G}$ and $\mathcal{E}$ cannot be empty. A BN cannot belong to both $\mathcal{B}^{A}$ and $\mathcal{B}^{I}$ at the same time. Each $B N$ $\mathcal{B}_{i} \in \mathcal{B}$ consists of a set of chance nodes $V\left(\mathcal{B}_{i}\right)$, potentially a set of utility nodes ${ }^{2} U\left(\mathcal{B}_{i}\right)$, and a set of directed edges.

The sets $\mathcal{B}^{A}$ and $\mathcal{B}^{I}$ from Definition 1 may contain different BNs at different times $t$. At any given time $t$, inference is carried out in the BNs in $\mathcal{B}^{A}$, thus they are participating in the current phase of the process and are partially responsible for whether the process stays in the same phase or moves to another phase. Intuitively a new phase starts when $\mathcal{B}^{A}$ changes, otherwise we say that we stay in the same phase. It is within the gates that criteria are defined which decide if a BN should stay active or should be deactivated. When drawing a GBN, all BNs that are active prior to any data points being supplied to the model have their names underscored (i.e. the initial set $\mathcal{B}^{\mathcal{A}}$ ). In Figure 2.6a for instance, Buy is active prior to any data points being supplied.

Definition 2 (Connections). The directed edges $\mathcal{E}$ connect either a node in $V\left(\mathcal{B}_{i}\right)$ or $U\left(\mathcal{B}_{i}\right)$ with a gate in $\mathcal{G}$, or a gate in $\mathcal{G}$ with an entire $B N$ in $\mathcal{B}$. An edge between a node and a gate is always directed away from the node towards the gate. An edge that connects a gate with an entire $B N$ can be directed either way.

Definition 3 (Parent/child). When a node is connected to a gate we consider the BN to which the node belongs to be a parent of the gate. When an entire BN and a gate are connected, the direction of the edge decides the parent/child relationship (the edge is directed towards the child).

In Figure 2.6a the edge from the chance node $E C_{B}$ to the gate $G_{1}$ implies that the BN Buy is a parent of $G_{1}$, while the edge from $G_{1}$ to the BN Sell implies that Sell is a child of $G_{1}$. In Figure 2.6c the edge between $R_{1}$ and $G_{1}$ defines $R_{1}$ as a parent of $G_{1}$, and the edge between $G_{1}$ and $R_{2}$ defines $G_{1}$ as a parent of $R_{2}$.

Definition 2 and Definition 3 also allow for a temporal order semantic to be given to the edges in $\mathcal{E}$. A process moves in the direction of the edges, where the gates define points where certain criteria must be met until the

\footnotetext{
${ }^{2} \mathrm{BNs}$ that are extended with utility and decision nodes are usually known as influence diagrams. We do not adopt the entire framework of influence diagrams, we only use the utility node to map variables' states to real values. Therefore we use the term BN rather than influence diagram.
} 
process can continue. Therefore, it is the data available at time $t$, together with the BNs in $\mathcal{B}^{A}$ and the gates that decide if the process stays in the current phase, or moves into a new phase in $t+1$. How the criteria in the gates are defined and met is explained by the following three definitions.

Definition 4 (Trigger node). A node that is connected with a gate is called a trigger node. All nodes that are connected to a gate make up the gate's trigger nodes. Trigger nodes that belong to active BNs supply values to the gates that they are connected to, specifically:

- For trigger nodes that are chance nodes: the posterior probability of the random variable taking a specific value, given some data.

- For trigger nodes that are utility nodes: the utility values weighted by the joint posterior distribution of the utility node's parents, given some data.

Definition 5 (Trigger network). All parent and child BNs of a gate make up the gate's trigger networks. Trigger networks supply values to gates that are computed by some function $f$, which takes as input a BN and some data.

Definition 6 (Trigger logic). Each gate $\mathcal{G}_{i}$ in $\mathcal{G}$ defines its own trigger logic, denoted as $T L\left(\mathcal{G}_{i}\right)$. The trigger logic is a logical statement regarding the values supplied by the gate's trigger nodes and trigger networks. When the trigger logic of a gate is satisfied, it is said to trigger.

Definitions 4, 5and 6 complete the structural definitions by defining how the criteria for the process to move forward are formed. Exactly how this is executed will be described in Section 2.2.3. However, it should be clear that the BNs that at time $t$ are in $\mathcal{B}^{A}$ are driving the current phase, supplying values to the gates, and when the trigger logic for one or more gates is satisfied, the temporal process moves forward to another phase. To clarify how the trigger logic can be formed, we here give three examples, utilising the GBNs in Figure 2.6a, 2.6b and 2.6c:

- $E C_{B}$ is a trigger node for $G_{1}$ in Figure 2.6a, and assuming that $E C_{B}$ has some state positive, $G_{1}$ could define its trigger logic as: $T L\left(G_{1}\right)$ : $p\left(E C_{B}=\right.$ positive $\left.\mid d_{t}\right)>\tau$, where $d_{t}$ is the data point available at time $t$ and $\tau$ is some threshold.

\footnotetext{
${ }^{3}$ In our applications, a typical choice for $f$ is the likelihood of a BN given some data.
} 
- In the GBN in Figure 2.6b, assume that $E C_{B}$ has six states, and that the utility node $U_{B}$ maps each one of $E C_{B}$ 's states to some real utility value. Now we can use $U_{B}$ as a trigger node for $G_{1}$ rather than $E C_{B}$, and let the trigger logic of $G_{1}$ be a statement of the utility value weighted by the posterior distribution of $E C_{B}$, i.e. $T L\left(G_{1}\right)$ : $\sum_{i=1}^{6} p\left(E C_{B}=i \mid d_{t}\right) U_{B}\left(E C_{B}=i\right)>\tau$.

- $R_{1}$ and $R_{2}$ are trigger networks of $G_{1}$ in Figure 2.6c. Given a function $f\left(\mathcal{B}_{i}, \mathcal{D}\right)$, where $\mathcal{D}$ represents some data set, the trigger logic for $G_{1}$ could be defined as $T L\left(G_{1}\right): f\left(R_{2}, \mathcal{D}\right) / f\left(R_{1}, \mathcal{D}\right)>\tau$. In this case the trigger logic is a comparison between $R_{1}$ 's and $R_{2}$ 's values given $f$ and $\mathcal{D}$, and if the division yields a value greater than some threshold $\tau$, then the gate triggers.

\subsubsection{Strategy encoding and decisions}

GBNs are not strictly decision models; possible decisions, actions and potential outcomes are not made explicit in the model. However, the structural definitions in Section 2.2.1 allow us to view GBNs as encoding a strategy, and this strategy can be mapped to a set of decisions.

In order to clarify this, let $\mathbf{D}$ be the set of every possible data set that can be presented to the GBN. The trigger logic of each gate then maps each set in $\mathbf{D}$ to either true or false, given the current BNs in $\mathcal{B}^{A}$. Specifically, let $\delta_{i}$ be the mappings that the trigger logic of gate $i$ defines, we then have $\delta_{i}: \mathcal{B}^{A} \times \mathbf{D} \rightarrow\{$ true, false $\}$. We can then define the strategy that a GBN encodes as $\Delta=\left\{\delta_{i}, i=1, \ldots, n\right\}$, where $n$ is the number of gates in the GBN. It is then clear that a GBN only encodes a strategy for when to trigger gates. This strategy will be followed as new data is collected and presented to the model (exactly how will be explained in Section 2.2.3).

However, it is possible to map the strategy that a GBN encodes to a set of decisions, e.g. for the GBN in Figure 2.6a we can define which decision to take by the decision function in Equation 2.12. In this example we map each data set $\mathcal{D} \in \mathbf{D}$ that we observe to a decision, given the current active BNs.

$$
\text { Decision } \mid \mathcal{D}=\left\{\begin{array}{l}
\text { Buy if } \delta_{1}\left(\mathcal{B}^{\mathcal{A}}, \mathcal{D}\right) \\
\text { Sell if } \delta_{2}\left(\mathcal{B}^{\mathcal{A}}, \mathcal{D}\right) \\
\text { Do nothing if none of the above apply }
\end{array}\right.
$$

An alternative way of defining this decision function, one that is more manageable from an operational standpoint, is to say that given a set of trig- 
gered gates $\mathcal{T}$, we return a decision depending on which gates that triggered, as in Equation 2.13. Since GBNs allow for multiple gates to trigger at the same time, consideration of such cases must be taken when defining the decision function. For instance, the function in Equation 2.13 could be expanded to also state that if both $G_{1}$ and $G_{2}$ triggers, then it should be considered as a signal that the model is ambivalent regarding the market, and thus no decision should be made at all.

$$
\text { Decision } \mid \mathcal{T}=\left\{\begin{array}{l}
\text { Buy if } G_{1} \in \mathcal{T} \\
\text { Sell if } G_{2} \in \mathcal{T} \\
\text { Do nothing if none of the above apply }
\end{array}\right.
$$

\subsubsection{Execution of a gated Bayesian network}

Having defined the structural definitions and explained how decisions can be read from the GBN, we continue by explaining how the model is to be executed over a set of data. Here we will offer one additional definition that is an integral part of the execution, and then define an execution algorithm that formalises how data points are sequentially entered into the model, and how the model reacts given the data.

Definition 7 (Activation and deactivation). When a gate triggers (see Definition 6), it activates all its child BNs and deactivates all its parent BNs. If several gates trigger due to the same set of data, then the union of all child $B N$ s are activated and the union of all parent BNs minus the union of all child $B N$ s are deactivated.

$$
\begin{aligned}
& U C B N=\text { Union of all child BNs of triggered gates } \\
& U P B N=\text { Union of all parent BNs of triggered gates } \\
& B N s \text { to activate = UCBN } \\
& B N \text { s to deactivate }=U P B N \backslash U C B N
\end{aligned}
$$

Figure 2.7 represents a high-level outline of the execution algorithm, which we shall describe here, and then subsequently give a detailed description of the algorithm. Given a set of sequential data points $\left[d_{1}, \ldots, d_{t}, \ldots, d_{T}\right]$, the algorithm starts by instantiating the variables of all active BNs with the first data point $d_{1}$. As was mentioned in the comment to Definition 1, which BNs that are initially active is defined when the model is created. The trigger logic for each gate is then checked, and if it is satisfied for any of the gates, the child BNs of these gates are activated (according to Definition 7 ). 


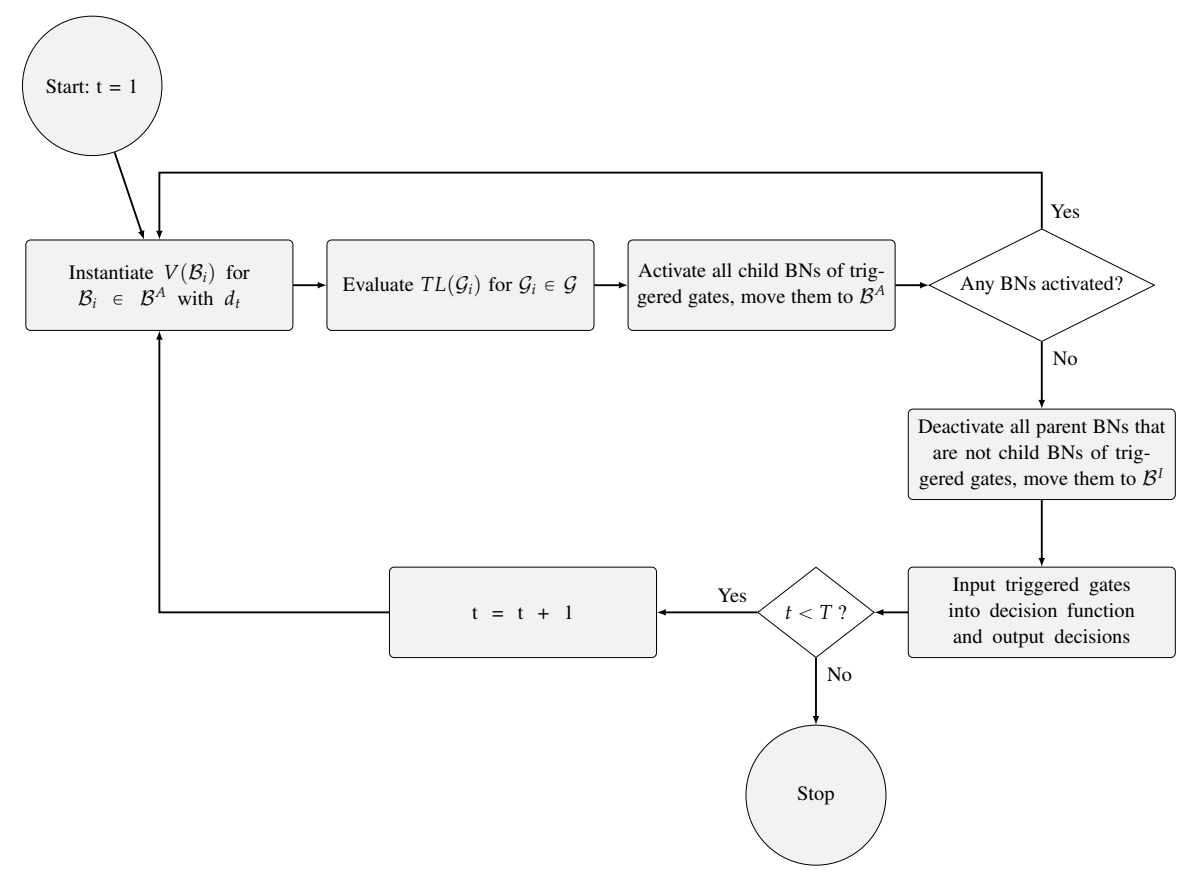

Figure 2.7: High-level outline of the execution algorithm.

If any BNs were activated, then the algorithm goes back and instantiates all variables of active BNs with the current data point, checks the trigger logic of gates and activates BNs. Once the previous loop does not result in any new BNs being activated, all parent BNs of triggered gates that are not child BNs of triggered gates are deactivated (according to Definition 7). GBNs are allowed to contain cycles, however as deactivations only occur once all activations have been handled, the execution algorithm will always terminate, and no infinite loops will be created. If a decision function has been defined, then the set of triggered gates are used as input to the decision function, and any returned decisions should be executed.

If there exists more data points, then $t$ is incremented and the next data point is processed. The active/inactive state of each $\mathrm{BN}$ is remembered between data points. Variables in inactive BNs are never instantiated according to new data points. A variable is instantiated according to some data point until a new data point instantiates it to a different state, thus evidence is never retracted from variables. The execution algorithm terminates when no more data points exist. 


\section{Execution algorithm}

In Figure 2.8, a detailed description of how the execution algorithm processes a sequentially ordered data set $\mathcal{D}$ is given. On line 3 , the outer loop starts that picks out the current data point $d_{t}$ and adds it to the currently available data $\mathcal{C}$. The currently available data is then passed to the EVIDENCE function. The result of the function call is a set of gates that triggered due to $\mathcal{C}$. These are used as input to an externally defined decision function on line 6 (as discussed in Section 2.2.2, which returns the decisions to take. On line 15, inside the function EVIDENCE, the inner loop of the algorithm starts. In each iteration, variable instantiations are updated for all active BNs according to the latest data point added to $\mathcal{C}$. Variables that were previously instantiated, but for which no new evidence has been supplied, keep their instantiation. The algorithm then finds those gates that have not yet triggered and sends them to the TRIGGER function on line 32, along with $\mathcal{C}$. The function will loop over the gates that have not yet triggered, evaluate their trigger logic, and if it is satisfied, adds the gate to the set of triggered gates. This set of triggered gates is then returned to the calling function and these will be added to the set $A T G$, which contains all the triggered gates. For each of the gates that triggered during this iteration of the loop (that started on line 15) their parent and child BNs are stored. Before the loop starts again, all child BNs that belong to triggered gates are activated. This is done in order to not enforce any ordering of the gates, so we can check the trigger logic for the gates in any order, and the same gates will trigger regardless. As long as there are gates that trigger the loop will continue. Once the loop is done, all BNs that are parents of gates that triggered, but are not children of any triggered gates, are deactivated. The deactivation is done outside the loop for the same reasoning of unordered gates previously mentioned. Finally all triggered gates are returned.

Notice that on line 19 we are creating a set of gates that belong to the GBN but have not yet triggered. It is this set of gates that are sent to the TRIGGER function on line 20. So once a gate has triggered it cannot be triggered again. Therefore the algorithm will always terminate, if not before then at least once all gates have triggered. This prevents any form of oscillation or infinite loop of triggering gates to happen.

Variables that are in inactive BNs will not be instantiated according to new data points. Since it is difficult for the user to predict which BNs will be activated on line 26, it is important that data points are given in their entirety to the model each time new data has been collected, even for variables for which the evidence might not have changed since the last data point. For 
instance, assume that a variable $A$ belongs to an inactive $\mathrm{BN}$ at time $t=1$. At this time new evidence is observed for $A$, however since it does not belong to an active $\mathrm{BN}$ it will not be instantiated with this new evidence. Now assume that at $t=2$, the $\mathrm{BN}$ that $A$ belongs to has become active but the available evidence for $A$ has not changed since $t=1$. Even though the evidence has not changed for $A$ between $t=1$ and $t=2$, it should be supplied to the model as it should not be required by the user to remember which BNs that have been inactive at which times in the past. 


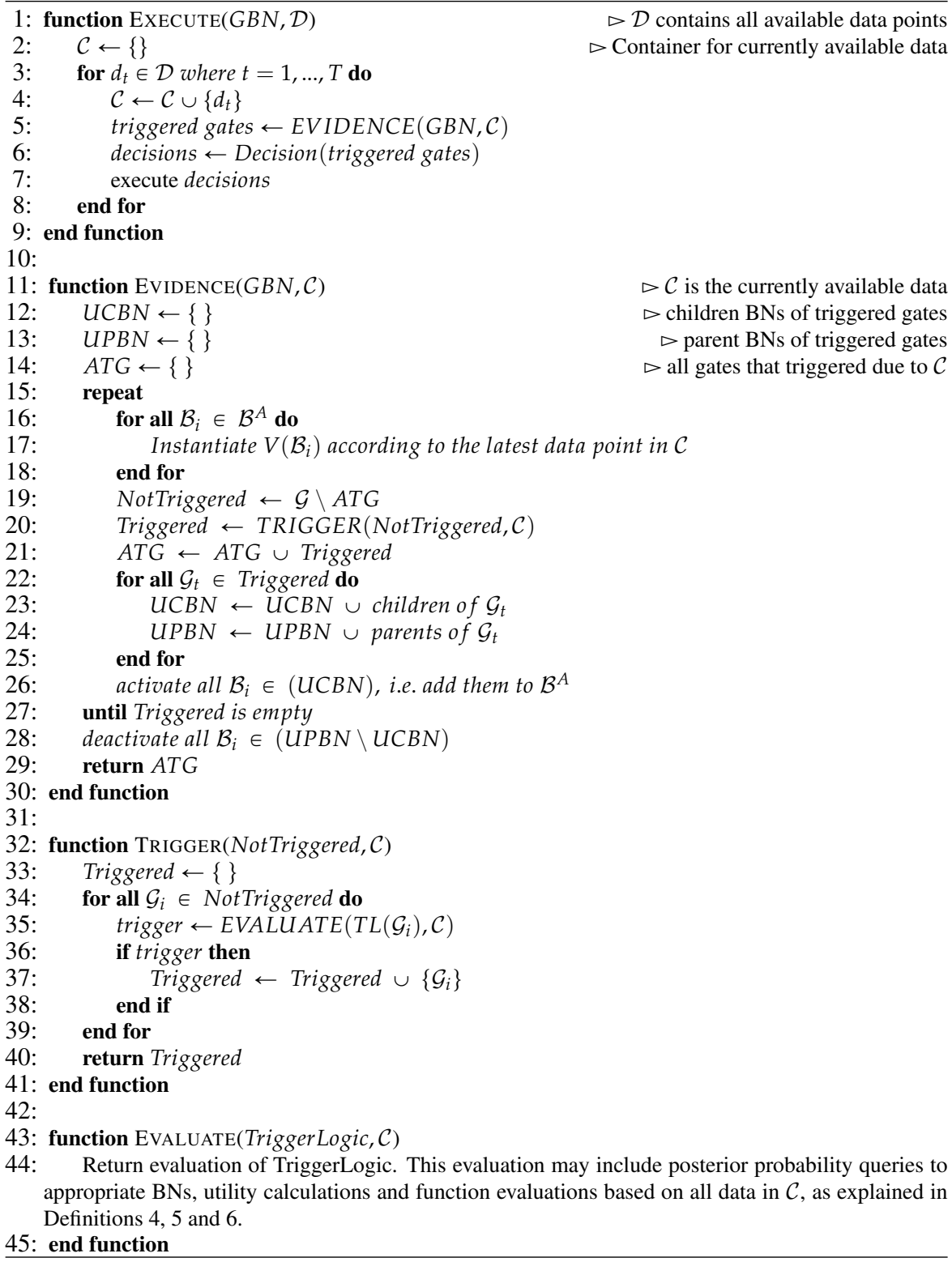

Figure 2.8: Pseudocode for the execution algorithm.

\subsubsection{Execution and modelling examples}

In order to put into context the structural definitions presented in Section 2.2.1 and the execution algorithm presented in Section 2.2.3, we will in this section demonstrate the application of the execution algorithm in the domain of the initial motivation for GBNs, namely trading financial assets. We will also 
give an illustrative example of how GBNs can be used in a different domain, showing the potential of GBNs as the number of phases increase. Finally, we shall give an example of using GBNs to represent independence model regimes, which will become a central theme in Chapters 4, 5 and 6 .

\section{The trader's problem}

The trader's problem is the scenario, in its simpler form, that initially motivated us to define GBNs, and a precursor to the real-world applications that will be presented in Chapter 3 . Assume that a trader wants to buy shares of a company when there is a belief that the share price will increase (i.e. there is a positive economical climate for the company under consideration). If the trader owns shares of the company then the trader wants to sell the shares if there is a belief that the share price will decrease (i.e. there is a negative economical climate for the company under consideration). The trader's problem is to decide when to move back and forth between the two phases of buying and selling shares, in such a way that it benefits the trader (what constitutes being beneficial will be discussed in Section 3.1.1.

The scenario can be modelled using the GBN depicted in Figure 2.6a. Here, $X$ and $Y$ are some features that predict the economical climate $E C_{B}$ during the identification of buying opportunities. Similarly, $W$ and $Z$ predict the economical climate $E C_{S}$ during the identification of selling opportunities. While the variables $E C_{B}$ and $E C_{S}$ may in this case represents the same realworld phenomenon regardless of the current phase, the posterior probability for these two variables will be computed at different times using different data. Also, $E C_{B}$ and $E C_{S}$ represent a future state, thus they are only observable with a time lag in a real-world setting. The variables $X, Y, W$ and $Z$ come before $E C_{B}$ and $E C_{S}$ in temporal order, therefore the edges are directed away from the predicting variables. This allows us to directly model the conditional probabilities $p\left(E C_{B} \mid X, Y\right)$ and $p\left(E C_{S} \mid W, Z\right)$. However, this is only tractable if very few predictor variables are considered, if the number of predictor variables were to increase, then alternatives should be explored in order to reduce the number of parameters in the model, for instance by using BN classifiers [18]. We shall explore such cases in Section 3.4 .

$G_{1}$ is programmed with trigger logic that defines when the trader wants to buy shares, in this example we will use $T L\left(G_{1}\right): p\left(E C_{B}=\right.$ positive $\left.\mid d\right)>$ 0.8 , where $d$ is a given data point. $G_{2}$ defines when the trader wants to sell shares, in this example we will use $T L\left(G_{2}\right): p\left(E C_{S}=\right.$ negative $\left.\mid d\right)>0.6$. A line under the name of the BN Buy indicates that it is active prior to any data being entered into the model (thus we are assuming that the trader does 
not own any shares at the beginning of the process). We will use the decision function in Equation 2.13 .

As is evident, the two decisions to buy and sell shares are dependent on different phenomena ( $X, Y, W$ and $Z$ ). Furthermore, we can program the trigger logic in such a way that we can be more sensitive to negative climate (using a lower threshold of 0.6) and less sensitive to positive climate (using a higher threshold of 0.8 ). This is one way of modelling the trader's preferences.

Assume that all variables are binary and that the following data set will be presented to the model:

- $d_{1}:\{\mathrm{X}=1, \mathrm{Y}=0\}$

- $d_{2}:\{\mathrm{X}=1, \mathrm{Y}=1, \mathrm{~W}=0\}$

- $d_{3}:\{X=1, Y=0, W=0, Z=1\}$

- $d_{4}:\{X=1, Y=0, W=1, Z=1\}$

The execution algorithm will then work as follows:

- $d_{1}$ : Variables $X$ and $Y$ belong to an active $\mathrm{BN} B u y$, and so they are instantiated according to $d_{1}$. Assume that this infers $p\left(E C_{B}=\right.$ positive $\left.\mid d_{1}\right)<0.8$, and so $T L\left(G_{1}\right)$ is not satisfied and thus $G_{1}$ does not trigger. Variable $E C_{S}$ belongs to an inactive $\mathrm{BN}$, and thus will not supply any posterior probability to $G_{2}$ (according to Definition 4), and therefore $T L\left(G_{2}\right)$ will not be satisfied. At this point in time we have not observed any evidence for variables $W$ and $Z$.

- $d_{2}$ : $X$ and $Y$ are updated as before. This time we will assume that $p\left(E C_{B}=\right.$ positive $\left.\mid d_{2}\right)>0.8$, satisfying $T L\left(G_{1}\right)$ and thereby triggering $G_{1}$. This will activate the BN Sell, and subsequently $W$ will be instantiated according to the evidence in $d_{2}$. Assume that $p\left(E C_{S}=\right.$ negative $\left.\mid d_{2}\right)<0.6$, then $G_{2}$ does not trigger. According to Definition 7, all parent BNs of triggered gates that are not children of triggered gates are deactivated. This implies that Buy is deactivated. Feeding the triggered gates into the decision function tells the trader that it is an opportune time to buy shares.

- $d_{3}$ : $W$ and $Z$ are updated according to $d_{2}$ as Sell now is active. Since $B u y$ is inactive, evidence for $X$ and $Y$ is discarded. Assume that $T L\left(G_{2}\right)$ is not satisfied, and due to Definition 4 we know that no posterior probabilities are supplied to $G_{1}$, thus $T L\left(G_{1}\right)$ cannot be satisfied. 
- $d_{4}: W$ and $Z$ are updated as before. This time we will assume that $p\left(E C_{S}=\right.$ negative $\left.\mid d_{4}\right)>0.6$, thus $T L\left(G_{2}\right)$ is satisfied and $G_{2}$ triggers. This will activate $B u y$, allowing $A$ and $B$ to be instantiated according to $d_{4}$. Assume that $p\left(E C_{B}=\right.$ positive $\left.\mid d_{4}\right)<0.8$, then $G_{1}$ does not trigger. This leads to Sell being deactivated. Feeding the triggered gates into the decision function tells the trader that it is an opportune time to sell shares.

\section{Patient monitoring}

In this section we will introduce an illustrative example of using a GBN in a different domain than that of financial asset trading. Here the number of phases involved has increased, and not all gates are mapped to an explicit decision. The example in this section also sets the stage for the comparison to other models done in Section 2.3. The GBN in Figure 2.9 models a process relating to a particular patient prior to and after surgery. Equation 2.14 defines the decision function for this GBN (where $\mathcal{T}$ represents a set of triggered gates).

$$
\text { Decision } \mid \mathcal{T}=\left\{\begin{array}{l}
\text { Perform surgery if } G_{3} \in \mathcal{T} \\
\text { Discharge patient if } G_{4} \in \mathcal{T} \\
\text { Readmit patient if } G_{5} \in \mathcal{T} \\
\text { Give antibiotics if } G_{6} \in \mathcal{T} \\
\text { Perform surgery if } G_{7} \in \mathcal{T} \\
\text { Stop monitoring if } G_{8} \in \mathcal{T} \\
\text { Do nothing if none of the above apply }
\end{array}\right.
$$

In the BN Normal risk monitoring, we only measure the patients temperature (Temp) and blood pressure (Blood) to decide whether or not it is appropriate to perform surgery. At the same time we are classifying the patient as either being in a normal state or in a high risk state (using the variable State). If the posterior probability of being in a high risk state is above some threshold, then $G_{1}$ will trigger, thus activating the $\mathrm{BN}$ High risk monitoring and deactivating Normal risk monitoring. When the patient is in the high risk state we also check the heart rate of the patient (Heart) to decide if it is time to perform surgery. Meanwhile, we are monitoring the risk/normal state of the patient, and if the posterior probability of the patient being in the normal state is above some threshold then $G_{2}$ will trigger, thus switching back and forth between the two monitoring phases. Notice that the triggerings of 


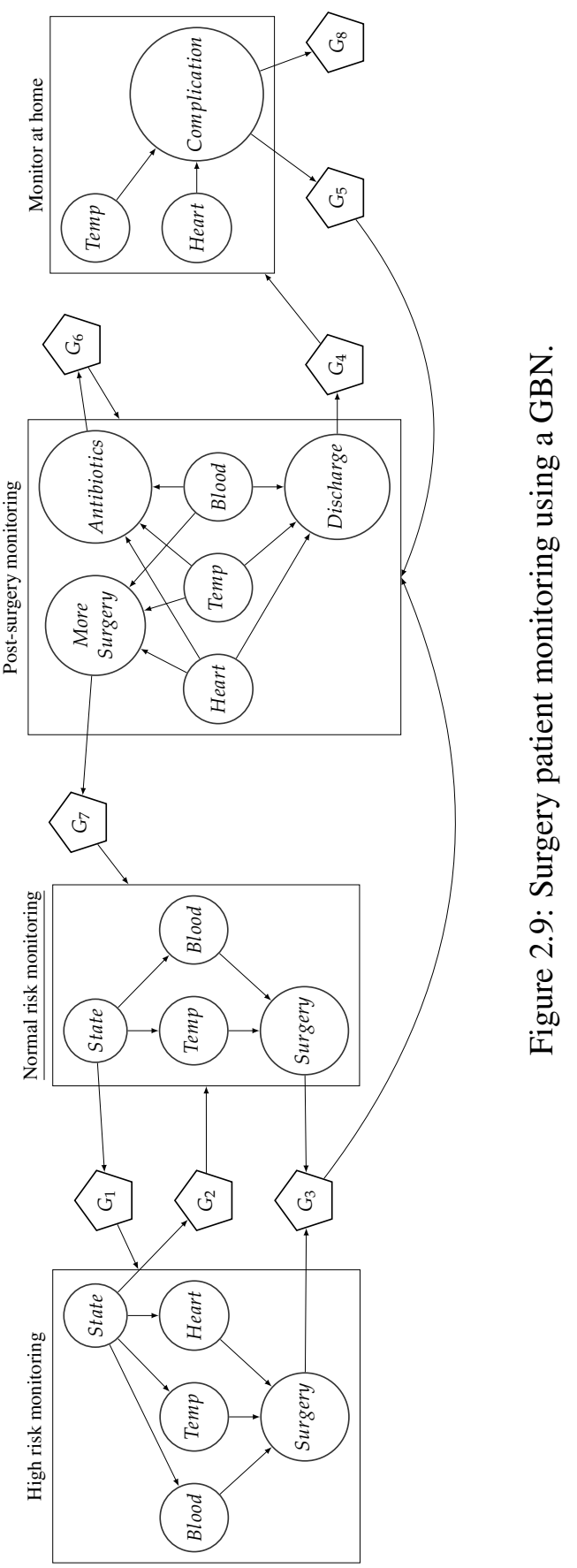


$G_{1}$ and $G_{2}$ do not lead to any explicit decisions according to the defined decision function (however in this specific case it is implicitly necessary for somebody to add or remove the heart rate monitoring device, unless it is always connected but not used).

At any time the posterior probability of Surgery $=$ true can exceed the threshold of $T L\left(G_{3}\right)$, thus indicating that it is appropriate to perform surgery, triggering the gate and deactivating both Normal risk monitoring and High risk monitoring, and activating the BN Post-surgery monitoring. In this example some of the networks are using the same variables and the decision stays the same (whether or not to perform surgery), however the conditional probabilities of the variables are different, and it could also be the case that the threshold is different in $G_{3}$ depending on which Surgery variable is supplying the posterior, e.g. let Surgery Normal $_{\text {be }}$ the Surgery variable in Normal risk monitoring and Surgery $_{H i g h}$ the variable in High risk monitoring, then it would be possible to define $T L\left(G_{3}\right)$ : $p\left(\right.$ Surgery $\left._{\text {Normal }} \mid d\right)>0.6 \vee p\left(\right.$ Surgery $\left._{\text {High }} \mid d\right)>0.8$.

After surgery, in Post-surgery monitoring, three gates can trigger, each one associated with a decision. Either the trigger logic of $G_{7}$ is satisfied and the decision is made to have another round of surgery, thus coming back to the normal/high risk monitoring phases. If the trigger logic of $G_{6}$ is satisfied then a round of antibiotics is given to the patient, and the post-surgery monitoring continues. If the patient is deemed healthy enough to be discharged, then the trigger logic of $G_{4}$ will be satisfied, and the monitoring can continue at home using the BN Monitor at home. When the patient is at home the blood test has been removed, but the temperature and heart rate is still measured. In case there is a high posterior probability of complications at home, $G_{5}$ will trigger, thus sending the patient back to the post-surgery monitoring at the hospital.

If the patient is at home, and the posterior probability of a complication is very low, then $G_{8}$ will trigger, leading to the decision to stop monitoring the patient. The entire GBN comes to a halt, as $G_{8}$ has no child BNs and no more data is collected.

We will use the patient monitoring example to highlight some key differences to other models and formalisms in Section 2.3.

\section{Representing independence model regimes}

The examples that we have given so far have considered cases where we modelled different variables within the different BNs, and used one or more of these variables as trigger nodes for the contained gates. When we want to 


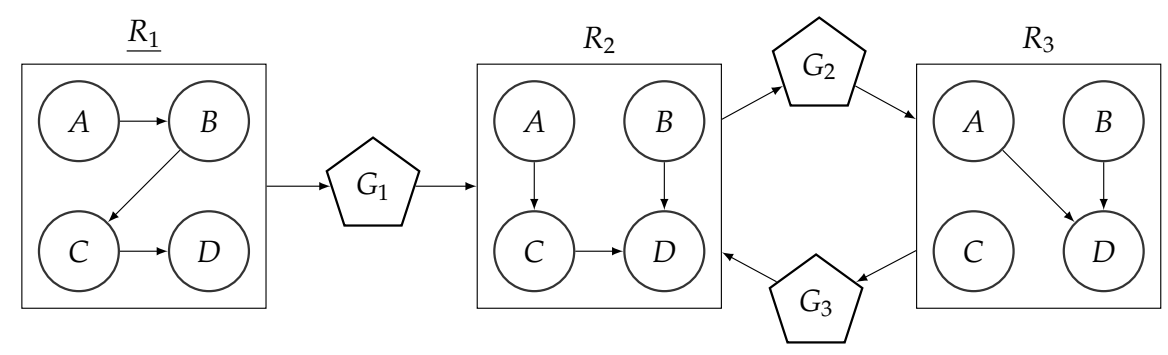

Figure 2.10: Modelling independence regimes using a GBN.

represent independence model regimes (which we shall define more clearly in Chapter 4, we consider the same variables in the BNs and rather let the edges change. In these cases we do not choose a subset of the modelled variables to act as trigger nodes, but rather allow the entire network to act as a trigger network. Thus, we must assess the appropriateness of the contained BNs as a whole.

Consider the GBN in Figure 2.10, and let there be a function $f(\mathcal{B}, \mathcal{D}) \in$ $\mathbb{R}$, where $\mathcal{B}$ is a $B N$, and $\mathcal{D}$ a data set over the variables in $\mathcal{B}$. The gates $G_{1}, G_{2}$ and $G_{3}$ define their trigger logic as a comparison between the value of $f$ given their child $\mathrm{BN}$ and the value of $f$ given their parent $\mathrm{BN}$. So for the GBN in Figure 2.10 we define the trigger logic of each gate according to Equation 2.15, where we require that the child's value divided by the parent's value be greater than some threshold in order for the gate to trigger. The function $f$ could for instance be the likelihood of the data $\mathcal{D}$ given a $\mathrm{BN}$.

$$
\begin{aligned}
T L\left(G_{1}\right) & :=\frac{f\left(R_{2}, \mathcal{D}\right)}{f\left(R_{1}, \mathcal{D}\right)}>\theta_{1} \\
T L\left(G_{2}\right) & :=\frac{f\left(R_{3}, \mathcal{D}\right)}{f\left(R_{2}, \mathcal{D}\right)}>\theta_{2} \\
T L\left(G_{3}\right) & :=\frac{f\left(R_{2}, \mathcal{D}\right)}{f\left(R_{3}, \mathcal{D}\right)}>\theta_{3}
\end{aligned}
$$

By using a GBN to represent a system that has several regimes, we can model each regime using a $\mathrm{BN}$, and then connect them with gates according to the system's regime transition structure (which will be defined and discussed in Chapter 4). We can also define the trigger logic in such a way that the model is capable of, given data points in a sequential order, accurately keeping the $\mathrm{BN}$ active that represents the regime the system currently is in. 


\subsection{Related formalisms}

Before we move ahead and discuss how GBNs can be learnt and used in an algorithmic trading context in Chapter 3, we shall take a step back and look at other related formalisms that have been proposed in the literature. Although as explained earlier, GBNs are not strictly decision models, they are nevertheless connected to a decision process. Therefore, we shall look at differences between GBNs and influence diagrams (IDs) and Markov decision processes (MDPs) in Section 2.3.1. Since GBNs also have the capacity to represent different regimes, or states of the world, we shall discuss differences with hidden Markov models (HMMs) in Section 2.3.2. We shall revisit HMMs in Chapters 4 and Chapters 6 for comparative reasons. There is also a connection between GBNs and models that explicitly take into consideration so called context specific independencies. We shall discuss briefly such connections in Section 2.3.3. In Chapter 7 we shall return to the concept of context specific independence. Finally, there are several other interesting lines of research that do have some connection to GBNs that we shall discuss in Section 2.3.4.

\subsubsection{Influence diagrams and Markov decision processes}

When the decisions, actions and possible outcomes can be made explicit in the model, then we usually consider decision models. IDs are, within the domain of probabilistic graphical models, the canonical models for dealing with decisions under uncertainty. An example of an ID is drawn in Figure 2.11a We express decisions and possible actions in a given scenario using decision nodes (square nodes) and express the value of each possible outcome using utility nodes (rhombus nodes). Given a fully specified ID we are able to extract the actions for each decision such that the expected utility is maximised, given evidence for the random variables [12]. This is referred to as finding the optimal strategy given the current evidence. This strategy can be computed due to the fact that all decisions and possible outcomes are explicitly modelled. If a decision needs to be repeated several times, it is possible to unfold the influence diagram, much like a DBN. It is then assumed that each decision is separated by a discrete time step that does not change in length, allowing chance and decision nodes to be connected between the time steps. Limiting the unfolding is the fact that it is necessary to explicitly bound the number of decisions in the process. Several obstacles when using IDs have been overcome, specifically concerning asymmetry in decision processes [19, 20].

In situations where it is necessary to model a decision process with an unbounded number of decisions, it is possible to use a MDP, an example 


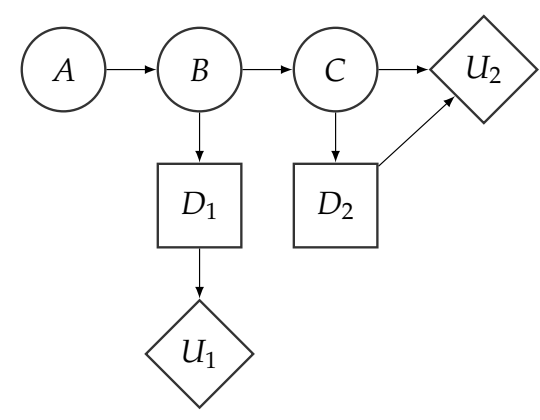

(a)
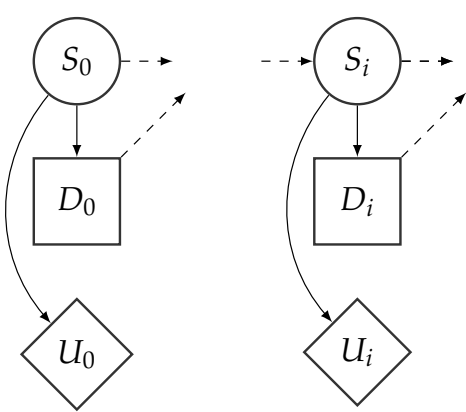

(b)

Figure 2.11: (a) An example of an ID, (b) an example of a MDP.

of which is shown in Figure 2.11b In the framework of MDPs, it is more common to phrase the problem as deciding to move between states, given the current state and available evidence, and that each decision changes the state with some probability. Being in a specific state is associated with some utility (which can be a reward if positive, or a cost if negative). Using value iteration, we can find a utility function that maps all states to a decision such that it maximises the expected utility. However, as the time horizon is not bounded, summing up utilities will lead to an infinite sum, and so in order for value iteration to work one must apply constraints on the model. A common way of doing this is to discount future utility, so that utilities in the distant future fades away. Value iteration finds the utility of a state by looking at the immediate utility received at that specific state plus the maximum expected utility of all future states that are reachable from that state [12]. Thus, if at time $j$ you can decide to move to a state where you own shares $s_{j+1}=$ own shares or a state where you do not own shares $s_{j+1}=$ do not own, then the utility function will map the current state to a decision of which state to move to, based on the maximum expected utility of all future states. However, the MDP and value iteration assumes that the utility of a state is independent of time $j$. This assumption becomes problematic when the utility depends on when and if we have made a decision at any previous time $i<j$. For instance, the value of our shares at time $j$ will be different if we bought them at time $i$ or time $k(i \neq k)$. This would require dynamically adding and removing edges across time in the MDP depending on previous decisions (as the utility function is no longer only a function of the current state).

In a GBN we do not explicitly model decisions and outcomes, and hence GBNs are not decision models and do not solve the maximum expected utility problem. Instead, the learning algorithms that we present in Chapter 3 finds 


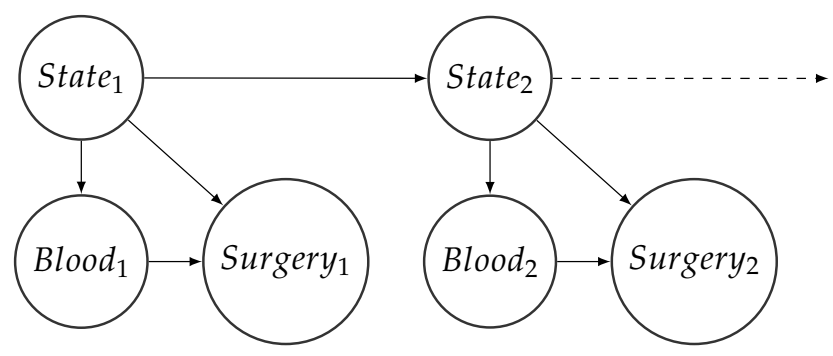

Figure 2.12: An example of a HMM.

the candidate which induces the best strategy $\Delta$ with respect to some score function $\mathcal{J}$. Put in other words, learning a GBN entails finding the candidate that encodes the strategy under which switching of BNs occur in such a way that the best score over a sequence of data points is achieved. It is clear that this is different from what IDs and MDPs achieve. GBNs repeat decisions similar to when folding out IDs, however it severs the ties between time slices, and can thus handle evidence at variable time steps (e.g. there is no need to specify that decisions are separated by one hour or one day, etc.). Also, it is not straightforward how one should learn the structure of an ID or an MDP from data. Furthermore, GBNs handle unbounded time horizons as MDPs do, however they do not use a discounting scheme or value iteration.

\subsubsection{Hidden Markov models}

In the patient monitoring example discussed in Section 2.2.4, we used a GBN to switch between two models based on the posterior probability of a patient being in a specific unobservable state. This is closely related to the way HMMs are used. However, there are a few fundamental differences that we will explore.

The HMM in Figure 2.12 is a simplified version of the scenario discussed. For this model, we would estimate from $\mathcal{D}$ the parameters of all the distributions implied by the model. We have to add an edge between State $t_{t}$ and

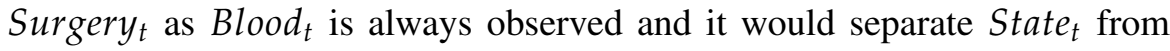
Surgery.

We have assumed that the patients state is not observable at the time of decision, hence the posterior of Surgery $t$ will be influenced by all other variables in the HMM, for all time steps. The HMM effectively becomes a mixture model, where the posterior of Surgery $y_{t}$ is weighed by the likelihood of each state at each previous time step. Now assume that we could observe the 
state of the patient at $t=2$. The posterior of Surgery $_{2}$ is now only influenced by State $_{2}$ and Blood $_{2}$.

In the GBN, we do not estimate what is known as the transition model $p\left(\right.$ State $_{t+1} \mid$ State $\left._{t}\right)$, thus we do not specify any conditional probability of activating and deactivating BNs. This conditional inherently exists due to the trigger logic and the threshold, however it is never explicitly specified.

The differences become even more apparent when considering the trader's problem, where we do not want the posterior of negative climate to become a mixture of all previous time steps, as some of the time steps should have been modelling positive climate. This ties into the fact that in the GBN it is straightforward to add and remove variables and dependencies based on which phase we are in. This could potentially be done in the HMM as well, however it would require some artificial augmenting of the nodes, edges and distributions, and as one of the main reasons of using a graphical model is to use it as a tool for communication, we prefer the explicit expressiveness of GBNs in this case.

We shall return to HMMs and have more to say about how they can be augmented to incorporate some of the features that exists in GBNs in Chapter 6.

\subsubsection{Context specific independence}

In the patient monitoring and independence model regime examples in Section 2.2.4. some variables are sometimes independent of each other and sometimes not, depending on the data that has been supplied, that is, depending on which BNs that are active. This is similar to what is known as context specific independence (CSI) [21], where previously dependent variables may become independent given some data (i.e. a context). The DAG representing a BN does not reveal these asymmetries, instead the conditional probability distributions must be estimated and investigated in order to identify them. CSIs have been studied in the domain of undirected graphical models and BNs [21, 22], but have also been studied in the domain of influence diagrams [23]. In [24], the authors give several examples of cases where CSIs may materialise, and they extend the variable elimination algorithm with the ability to utilise CSIs within the BN, resulting in more efficient inference. Enforcing CSI has also been used to facilitate more efficient inference and parameter estimation, for instance in the form of noisy OR-models [2, 25].

Chain event graphs (CEGs) were introduced as a probabilistic graphical model that represents these asymmetries explicitly, while at the same time being able to represent the BN in case of symmetry [26]. CEGs are defined 
as a function of an event tree, which represents how a certain process may unfold, and certain symmetries in this event tree. The symmetries are exploited to create a graph where steps in the process that are the same are collapsed. This not only decreases the size of the event tree, but also makes explicit the asymmetric relationships among steps in the process. CEGs have since further been developed in order to model processes with non-finite event trees [27.

A distinct difference between CEGs and GBNs is the fact that CEGs encode a probability of moving from one step to another in the event tree. GBNs do not encode this probability, i.e. it is not made explicit that with probability $\alpha$ the GBN in Figure 2.6a will transition from the Buy phase to the Sell phase. Instead, transitions in a GBN depend on the posterior probabilities inferred by the contained BNs over the observations made.

While GBNs also are capable of explicitly describing some of the asymmetries that CSI entails, it is quite different from those explained by [21] and [26]. Since GBNs use BNs to model the phases, asymmetries can still exist in these BNs that are not made explicit in the graph. GBNs make explicit asymmetries that occur once a process has changed phase, i.e. once a set of evidence has fulfilled the conditions of the trigger logic of some gate. These conditions are not necessarily fulfilled by one single variable taking one specific state, but is a joint condition on potentially several variables and the threshold of the trigger logic.

We shall have reason to return to the concept of CSI in Chapter7. There we shall use GBNs to explicitly model CSIs in such a way that we may use them as a means for causal effect identification.

\subsubsection{Other formalisms}

Taking a large BN and dividing it into smaller subnets has been researched for some time. Notable contributions include multiply sectioned Bayesian networks [28, 29], agent encapsulated Bayesian networks [30], and objectoriented Bayesian networks [31]. Although these frameworks all section the BNs into smaller parts, they still come together, unlike GBNs, to create one large $\mathrm{BN}$.

\subsection{Summary}

In this chapter we have introduced GBNs as an extension of BNs that allows us to model both distinct phases in a decision process and independence model regimes. We have introduced structural definitions that define how 
GBNs can be built, and offered an execution algorithm which can be used to process data through the GBN. We also explained the fundamentals of structure learning, parameter estimation and inference with regards to BNs. In the coming chapters we shall take steps towards turning the GBN model from being a theoretical idea, to becoming an operational model. For this we require a much more specific goal for the use of our GBNs, and a strategy for learning GBNs that can accommodate these goals. 


\section{Chapter 3 \\ Learning gated Bayesian networks for algorithmic trading}

In this chapter we shall look at two different approaches to learning GBNs, with the specific goal of algorithmic trading. In Section 3.2 we shall account for a template based approach, where a set of BNs and gates are supplied by experts, and then the candidate GBN which performs best is returned as the learnt GBN. In the second approach, described in Section 3.4, we attempt to do away with some of the expert knowledge required by the template based approach, moving towards a more fully automatic learning algorithm. In both cases we shall learn GBNs that trade financial assets, and therefore we begin this chapter with a general introduction to algorithmic trading.

\subsection{Introduction to algorithmic trading}

In Chapter 2 we discussed cases of a trader looking to buy and sell financial assets. While this is an intuitive way of looking at the problem at hand, more formally the process we intend to model is part of a larger process commonly referred to as algorithmic trading. Algorithmic trading can be viewed as a process of actively deciding when to own assets and when to not own assets, so as to get better risk and reward on invested capital compared to holding on to the assets over a long period of time. At the other end of the spectrum is the buy-and-hold $(\mathrm{BaH})$ strategy, where one owns assets continuously over a period of time without making any decisions of selling or buying during the period.

An algorithmic trading system consists of several components, some of which may be automated by a computer, and others that may be manually 


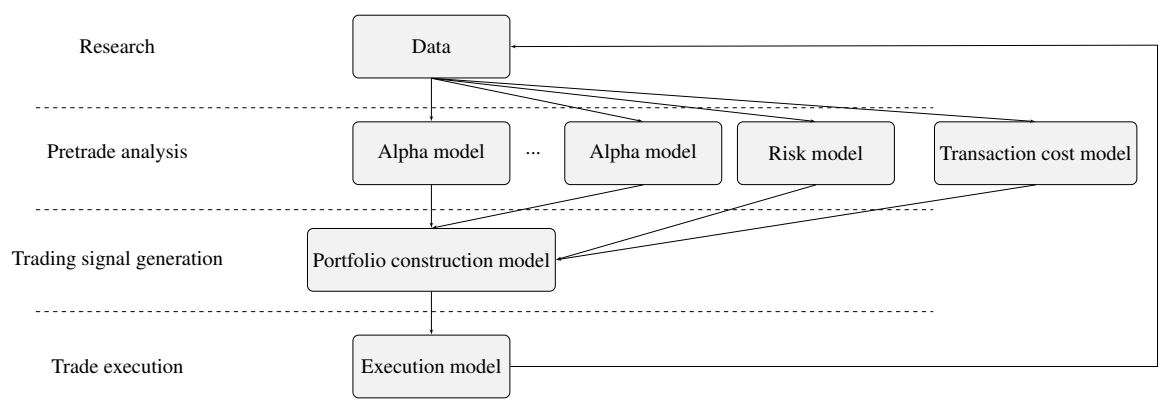

Figure 3.1: Components of an algorithmic trading system.

executed [32, 33, 34]. A schematic overview of the components of a general algorithmic trading system is shown in Figure 3.1 .

The type of data used at the research stage varies greatly, e.g. net profit, potential prospects, sentiment analysis, analysis of previous trades, or technical analysis, which will be the focus in the applications of this chapter. The analysis of the data is split up into alpha, risk and transaction cost models. The alpha models are responsible for outputting decisions for buying and selling assets based on the data they are given. These decisions are known as buy and sell signals, examples of which are depicted in Figure 3.2 (an arrow pointing upwards is a buy signal and a downwards facing arrow is a sell signal, the signals are drawn on top of the assets historical price). If followed, these buy and sell signals give rise to certain risk and reward on the initial investment (which will be described further in Section 3.1.1.

The risk and transaction cost models should be seen as strategies for managing risk and transaction costs in a system that has many alpha models. The output from these three types of models (alpha, risk and transaction) are in their turn the input to the portfolio construction model in the trading signal generation stage. Here the output of the previous components are combined to decide which signals to actually execute in order to create a portfolio that is based on a combination of alpha models. These signals can lead to decisions to buy more of a certain asset, to sell all or a portion of assets already owned, or in some cases to short certain assets so that reward is achieved when the asset loses value. Portfolio construction is a widely researched topic that has been approached from both the financial field and from an information theoretic perspective. In finance, the most common basis is the theory of mean-variance portfolios, also known as Markowitz portfolio theory [35, 36], where the tradeoff between expected risk and reward is used to allocate resources amongst a basket of assets. While from an information theoretic background the focus has been on online portfolio construction algorithms 


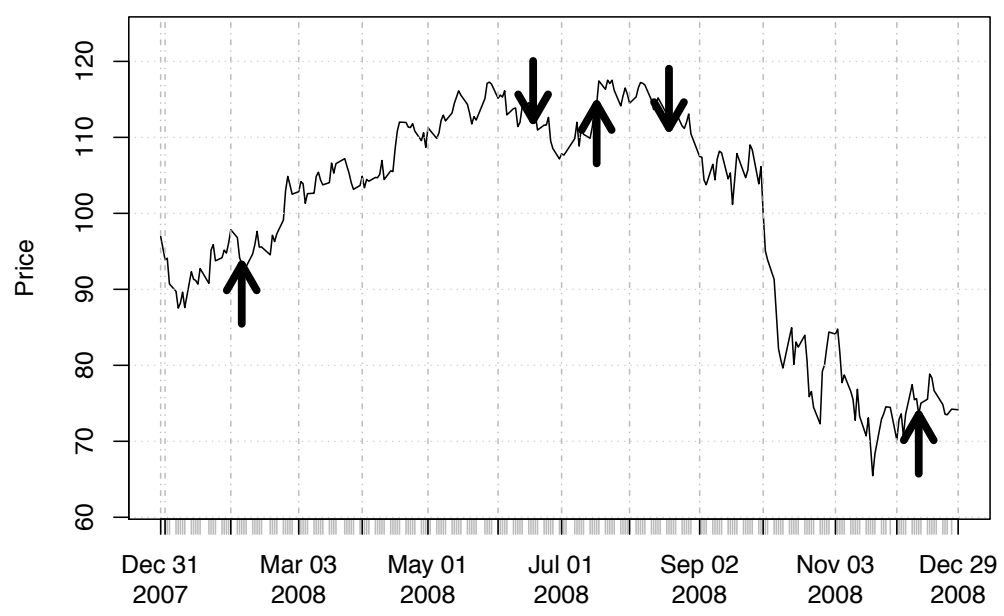

Figure 3.2: Price of an asset with buy and sell signals overlaid.

[37], such as the universal portfolio [38] and the exponential gradient [39], where resources are initially allocated equally, but sequentially are changed according to varying criteria that in the long run creates optimal growth.

The final stage is the actual execution of the trading signals, which must be done in a manner that does not affect the price of the asset that is being bought. Although all components are important we will not be addressing them all, instead our contribution is concerned with the use of GBNs as alpha models (informally the trader buying and selling shares can be seen as an alpha model).

\subsubsection{Evaluating alpha models}

Regression models can be evaluated by how well they minimise some error function or by their predictive scores. For classification, the accuracy and precision of a model may be of greatest interest. Alpha models may rely on regression and classification, but cannot be evaluated as either. For an alpha model, it is not important to accurately predict every movement of the market, but rather to identify events in the market that suggest an opportune time to buy or sell. Therefore, optimising alpha models by using classical supervised classification measures such as accuracy, precision, recall, etc. will not be in line with the desired behaviour of the model. To clarify, it is not necessarily known prior to learning when these opportune times are, and so the task is in this sense unsupervised, as there is no way of guiding the model to which events it should classify correctly. An alpha model's performance needs to be based on its generated signals over a period of time, and the performance 
must be measured by the risk and reward of the model. This is known as backtesting.

\section{Backtesting}

The process of evaluating an alpha model on historic data is known as backtesting, and its goal is to produce metrics that describe the behaviour of a specific alpha model. These metrics can then be used for comparison between alpha models [40, 41]. A time range, price data for assets traded and a set of signals are used as input. The backtester steps through the time range and executes signals that are associated with the current time (using the supplied price data) and computes an equity curve (which will be explained in the following section). From the equity curve it is possible to compute metrics of risk and reward. To simulate potential transaction costs, often referred to as commission, every trade executed is usually charged a small percentage of the total value $(0.06 \%$ is a common commission charge used in our applications).

Alpha models are backtested separately from the other components of the algorithmic trading system, as the backtesting results are input to the other components. Therefore, we execute every signal from an alpha model during backtesting, whereas in a full algorithmic trading system we would have a portfolio construction model that would combine several alpha models and decide how to build a portfolio from their signals.

\section{Alpha model metrics}

What constitutes risk and reward is not necessarily the same for every investor, and investors may have their own personal preferences. However, there are a few metrics that are common and often taken into consideration [41]. Here we will introduce the metrics that we will use to evaluate the performance of our alpha models.

Although not a metric on its own, the equity curve needs to be defined in order to define the following metrics. The equity curve represents the total value of a trading account at a given point in time. If a daily timescale is used, then it is created by plotting the value of the trading account day by day. If no assets are bought, then the equity curve will be flat at the same level as the initial investment. If assets are bought that increase in value, then the equity curve will rise. If the assets are sold at this higher value then the equity curve will again go flat at this new level. The equity curve summarises the value of 
the trading account including cash holdings and the value of all assets. We will use $\mathrm{Y}_{t}$ to reference the value of the equity curve at point $t$.

Metric 1 (Return). The return of an investment is defined as the percentage difference between two points on the equity curve. If the timescale of the equity curve is daily, then $r_{t}=\left(\mathrm{Y}_{t}-\mathrm{Y}_{t-1}\right) /\left|\mathrm{Y}_{t-1}\right|$ would be the daily return between day $t$ and $t-1$. We will use $\bar{r}$ and $\sigma_{r}$ to denote the mean and standard deviation of a set of returns.

Metric 2 (Sharpe Ratio). One of the most well known metrics used is the so called Sharpe ratio. Named after its inventor Nobel laureate William F. Sharpe, this ratio is defined as: $(\bar{r}-$ risk free rate $) / \sigma_{r}$. The risk free rate is usually set to be a "safe" investment such as government bonds or the current interest rate, but is also sometimes removed from the equation [41]. The intuition behind the Sharpe ratio is that one would prefer a model that gives consistent returns (returns around the mean), rather than one that fluctuates. This is important since investors tend to trade on margin (borrowing money to take larger positions), and it is then more important to get consistent returns than returns that sometimes are large and sometimes small. This is why the Sharpe ratio is used as a reward metric rather than the return.

Furthermore, under certain assumptions it can be shown that there exists an optimal allocation of equity between alpha models (in the portfolio construction model), such that the long-term growth rate of equity is maximised [41]. This growth rate turns out to be $g=r+S^{2} / 2$, where $r$ is the risk free rate and $S$ is the Sharpe ratio. Thus, a high Sharpe ratio is not only an indication of good risk adjusted return, but holding the risk free rate constant, the optimal growth rate is an increasing function of the Sharpe ratio.

Using the Sharpe ratio as a metric will ensure that the alpha models are evaluated on their risk adjusted return, however, there are other important alpha model behaviours that need to be measured. A family of these, that are known as drawdown risks, are presented here (see Figure 3.3 for examples of an equity curve and these metrics).

Metric 3 (Maximum Drawdown (MDD)). The percentage between the highest peak and the lowest trough of the equity curve during backtesting. The peak must come before the trough in time. The MDD is important from both a technical and psychological regard. It can be seen as a measure of the maximum risk that the investment will live through. Investors that use their existing investments that have gained in value as safety for new investments may be put in a situation where they are forced to sell everything. Other 


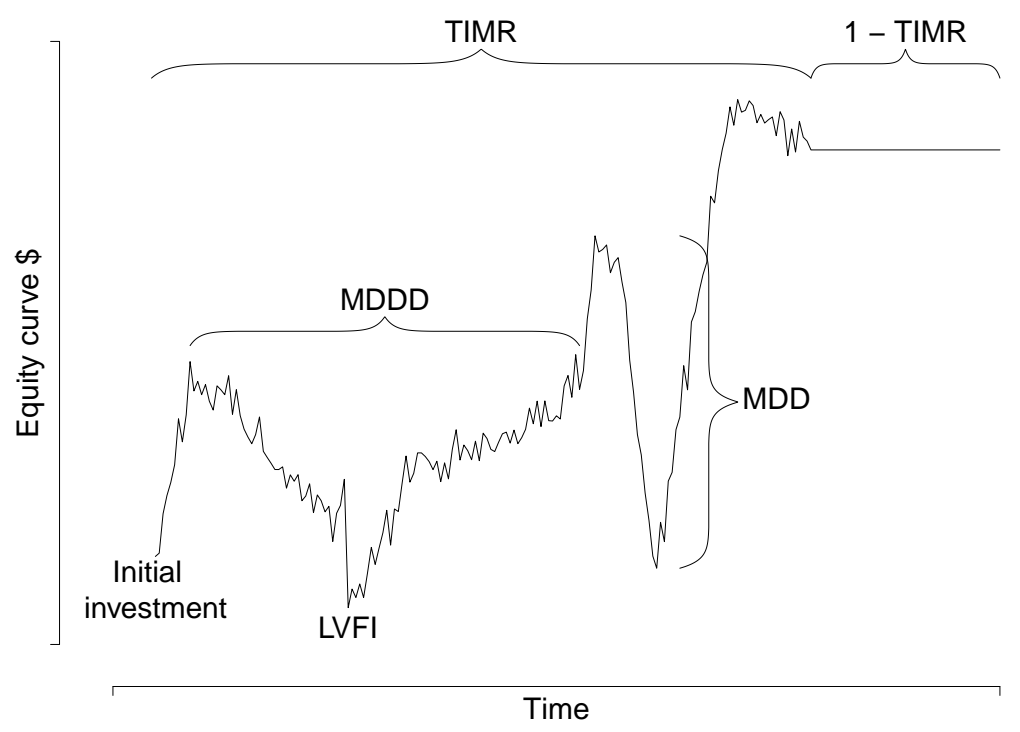

Figure 3.3: Example of an equity curve with drawdown risks.

risk management models may automatically sell investments that are losing value sharply. For the individual who is not actively trading but rather placing money in a fund, the MDD is psychologically frustrating to the point where the individual may withdraw their investment at a loss in fear of losing more money.

Metric 4 (Maximum Drawdown Duration (MDDD)). The longest it has taken from one peak of the equity curve to recover to the same value as that peak. Despite its unfortunate name it is not the duration of the MDD, but rather then longest drawdown period. There is an old adage amongst investors to "cut your losses early". In essence it means that it is better to take a loss straight away than to sit on an investments for months or years, hoping that it will come back to positive returns. During this time one could have reinvested the money elsewhere, rather then breaking-even much later (or taking a larger loss much later). Models that have long periods of drawdown lock resources when they could have been used better elsewhere.

Metric 5 (Lowest Value From Investment (LVFI)). The percentage between the initial investment and the lowest value of the equity curve. This is one of the most important metrics, and has a significant impact on technical and psychological factors. For investors trading on margin, a high LVFI will cause the lender to ask the investor for more safety capital (known as a margin call). This can be potentially devastating, as the investor may not have the capital 
required, and is then forced to sell the investment. The investor will then never enjoy the return the investment could have produced. Individuals who are not investing actively, but instead are choosing between funds that invest in their place, should be aware of the LVFI as it is the worst case scenario if they need to retract their investment prematurely.

Metric 6 (Time In Market Ratio (TIMR)). The percentage of time of the investment period where the alpha model owned assets. This metric may seem odd to place within the same family as the other drawdown risks, however it fits naturally in this space. We can assume that the days the alpha model does not own any assets the drawdown risk is zero. If we are not invested, then there is no risk of loss. In fact, we can further assume that our equity is growing according to the risk free rate, as it is not bound in assets.

\subsubsection{Benchmark}

At first the BaH strategy may seem naïve, however it has been shown that deciding when to own and not own assets requires consistent high accuracy of predictions in order to gain higher returns than the $\mathrm{BaH}$ strategy [42]. The $\mathrm{BaH}$ strategy has become a standard benchmark, not only because of the required accuracy, but also because it requires very little effort to execute (no complex computations and/or experts needed). In recent years it has been revealed that many UK equity funds labelled as "actively managed" have in fact been tracking the stock market [43], essentially creating an expensive $\mathrm{BaH}$ strategy for the investor. Furthermore, investors have been shifting to passive investments over the past ten years [44]. This adds to the evidence that passively holding a $\mathrm{BaH}$ strategy is hard to beat, as both investors and fund managers have resorted to this strategy rather than attempting to time the market.

\subsubsection{Technical analysis}

In the applications presented throughout this thesis we use data based on technical analysis as input to our alpha models. Technical analysis has its roots in a series of editorials that Charles Dow wrote for The Wall Street Journal that date to the late 19th century. In these writings Dow suggested methods to analyse the current economical climate using nothing but the action of stock prices [45, 46]. The year after Dow's death in 1902, Samuel A. Nelson compiled these essays into a book [47], suggesting the term Dow Theory. In 1922 William P. Hamilton took the principles Dow described and published them 
as the Dow Theory as it is commonly known today [48]. Robert Rhea furthered the work of Dow and published his developments of Dow Theory in 1932 [49].

The development of using the action of stock prices to forecast economical climate did not end there. In 1938, the Elliot wave principle was introduced by Ralph N. Elliot [50], a theory very much influenced by Dow Theory and especially by the work of Robert Rhea.

These original theories are all based around a few tenets (all of which we will not discuss here, but we refer the interested reader to the references). One of the major concepts is that the movement of price of an asset repeats itself in recognisable patterns. An analyst who becomes skilful in identifying these patterns, and correctly interpreting them, would be able to foresee (with some uncertainty) future price movements. Furthermore price moves in trends, both in the long and intermediate term. As such it is important for the analyst to identify if the current trend of the stock is upward, downward or just sideways. The concept of trend can be seen on a price chart, even an untrained eye should be able to spot times where the stock is clearly moving upwards, downwards or sideways. Major trends can last for years, whilst having intermediate (shorter trends) going the opposite direction for some time, only to later on continue the major trend. This structure, of major and intermediate trends, resembles waves (hence Elliot wave principle).

Today technical analysis has grown to accommodate several theories, including theories used by Japanese rice traders that predate Dow with a couple of centuries [51]. Advancements in availability and power of computers (beginning with general access to programmable calculators [52]), have allowed for massive development of so called indicators. These indicators are computations of price and volume that support the investor in identifying and confirming chart patterns used for forecasting.

Technical analysis can be seen as divided into two main areas: traditional charting and quantitative analysis. Traditional charting is more true to Dow's and Elliot's theories and the evolution of these, where the primary objective is using price and volume to visually identify patterns in charts. Quantitative analysis takes the principles of technical analysis and quantifies, test and optimises them, in an attempt to automate the process of generating buy sell signals. Quantitative analysts can further be categorised as those who develop systems that are fully automatic (where the details of the system are hidden from the investor) and those who develop new indicators that are supposed to help the investor make decisions [45].

In reality most technical analysts probably use a bit from both worlds, traditional chartists might use some indicators and computations to aid them, 
and quantitative analysts use traditional chartists' ideas to develop new systems. For the interested reader there are several good books on the matter (please see the references) and also publications addressing modern developments available online, such as the Journal of Technical Analysis [53] and IFTA Journal [54].

In our applications we use three common technical analysis indicators: the moving average (MA), the moving average convergence/divergence (MACD), and the relative strength index (RSI). The MA is the average price over time (using a sliding window with some predefined size), while the MACD is the relative difference between two MAs using different sized windows (where the MA with the smaller size window is often referred to as the fast MA, and the other the slow MA). The RSI compares the size of recent gains to the size of recent losses, becoming a gauge for which side of the trade has been stronger in the recent past (i.e. are buyers pushing up the price, or are sellers pulling down the price).

Calculations of the MA and MACD are given by Equation 3.1, where $S$ is a set of ordered values (i.e. a set of prices for some asset), $t$ is the time index for which we wish to calculate the indicator, and $n$ is the period, or the size of the window, of the MAs.

$$
\begin{aligned}
M A_{t}(S, n) & =\frac{1}{n} \sum_{i=0}^{n-1} S_{t-i} \\
M A C D_{t}\left(S, n_{\text {fast }}, n_{\text {slow }}\right) & =\frac{M A_{t}\left(S, n_{\text {fast }}\right)-M A_{t}\left(S, n_{\text {slow }}\right)}{M A_{t}\left(S, n_{\text {slow }}\right)}
\end{aligned}
$$

Calculating the RSI for a set of prices $S$ is done according to Equation 3.2. where $n$ represents the period (or the size of a sliding window). $R S_{t}$ represents the average gain divided by the average loss over the period $n$ (note that division by $n$ is cancelled out and therefore not present). The $R S_{t}$ value is then scaled to a range between 0 and 100 in order to get the $R S I_{t}$ value.

$$
\begin{aligned}
U p_{t}(S, n) & =\left\{\left|S_{i}-S_{i-1}\right|: S_{i}>S_{i-1} \text { with } t-n<i \leqslant t\right\} \\
\operatorname{Down}_{t}(S, n) & =\left\{\left|S_{i}-S_{i-1}\right|: S_{i}<S_{i-1} \text { with } t-n<i \leqslant t\right\} \\
R S_{t}(S, n) & =\frac{U p_{t}(S, n)}{\operatorname{Down}_{t}(S, n)} \\
\operatorname{RSI}_{t}(S, n) & =100-\frac{100}{1+R S_{t}(S, n)}
\end{aligned}
$$

In Figure 3.4 the price of an asset along with these indicators are plotted. The price of the asset is plotted in black, along with a MA in red. Below the 


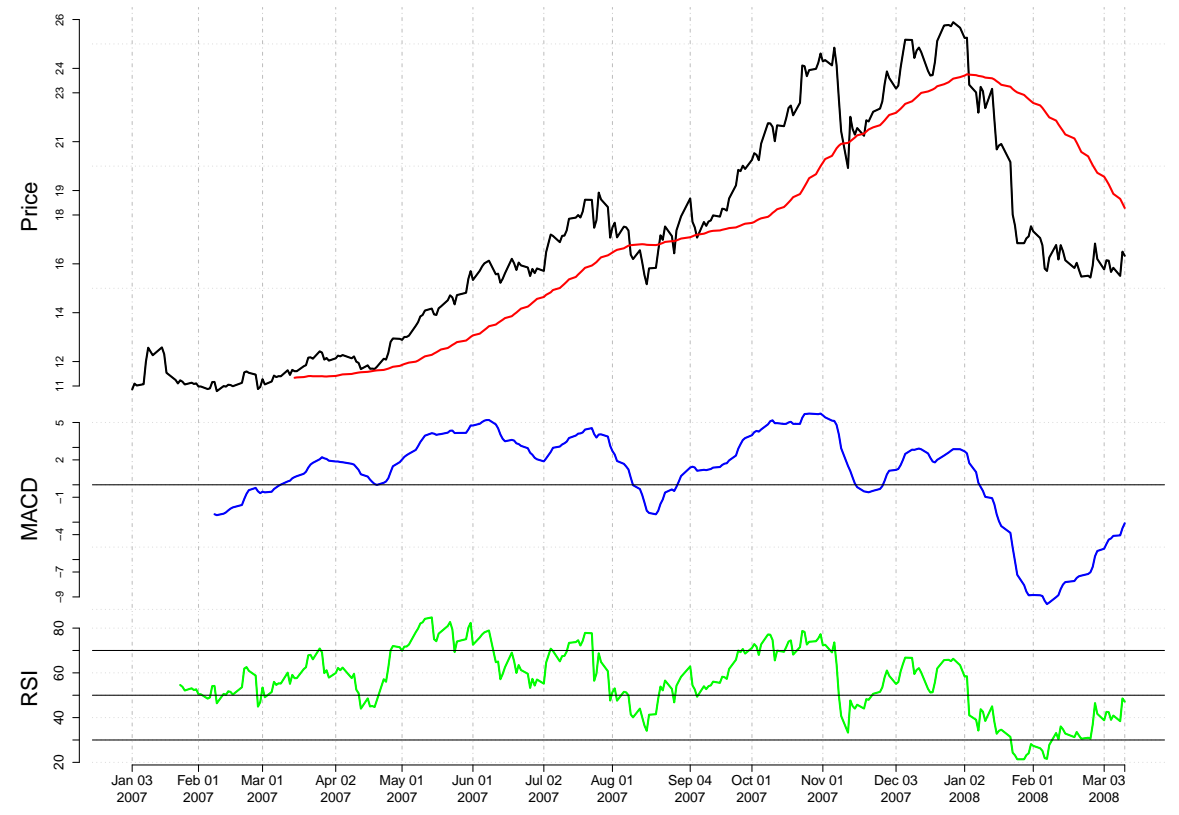

Figure 3.4: A trading screen showing the price of an asset (black) together with a MA (red). Below the price the MACD indicator (blue) and RSI (green) are plotted.

price the MACD is plotted in blue, with a horizontal line at zero to indicate when the slow MA is above/below the fast MA. The RSI is plotted in green, with horizontal lines at 30,50 and 70 (these values are guidelines that indicate which side of the trade, buy or sell, has been dominant).

\subsection{Template based learning}

Having introduced GBNs in Chapter 2 and algorithmic trading in Section 3.1 , we now turn our attention to how we can learn GBNs that act as alpha models. To this end we shall in this section describe the first of two learning algorithms that we have proposed, and show how it performs in a real-world financial trading situation. We call the algorithm template based, and use a library of BNs and gates to create a set of candidate alpha models. We then employ a combination of time series and $k$-fold cross-validation in order to select the best candidate. We shall begin by explaining the details of the algorithm, and then move on to the real-world experiments. 
Library for $\mathrm{G}_{2}$

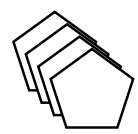

Library for $\mathrm{BN}_{1}$
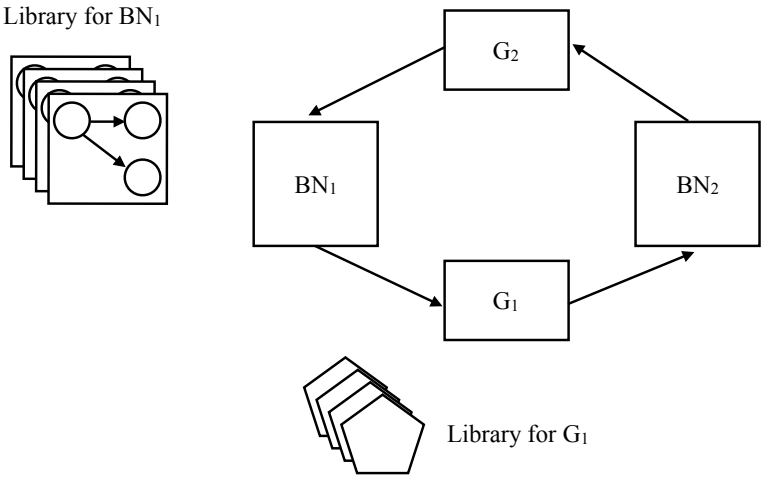

Library for $\mathrm{BN}_{2}$

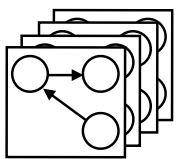

Figure 3.5: Example of a GBN template with two BN slots and two gate slots. Each BN slot has a library of pre-defined BN structures, which can be placed in each respective slot, similarly so for gates.

\subsubsection{Gated Bayesian network template}

A GBN template is a representation of the modelled phases, including the possible transitions between them. The template defines where BNs and gates can be placed. For each slot where a BN can be placed, there is a library of BNs to choose from, similarly so for gates (gates differ in their trigger logic, e.g. the thresholds may vary between them). BNs may be hand-crafted by experts prior to the GBN modelling, or they may be learnt from data using some structure learning algorithm. In any case, it is expected that the user provides the template and the libraries, hence the algorithm can be classified as semi-automatic. A template with four slots and corresponding libraries is depicted in Figure 3.5 .

Selecting a BN and a gate from the libraries for each slot in the template creates a GBN (e.g. Figure 2.6a), we call this a candidate of the template. We use $\mathcal{C}_{i}$ to denote GBN candidate $i$ of a GBN template. Since the structure of the BNs and the trigger logic of the gates in the libraries are defined, the remaining free parameters of a GBN candidate $\mathcal{C}_{i}$ are the parameters of the marginal and conditional probability distributions of the contained BNs.

The only restrictions on the BNs and gates are the ones they place on each other, e.g. if the trigger logic of the gates placed in $G_{2}$ includes an expression about the posterior probability of a negative economical climate, 
then the BNs placed in $B N_{2}$ must contain a node that can supply this value. Except for these restrictions, the BNs and gates can be configured freely.

\subsubsection{Multiple simulations}

A data set $\mathcal{D}$ of consecutive evidence sets, e.g. observations over all or some of the random variables in the GBN, is divided into $n$ equally sized blocks $\left(\mathcal{D}_{1}, \ldots, \mathcal{D}_{n}\right)$ such that they are mutually exclusive and exhaustive. Each block contains consecutive evidence sets and all evidence sets in block $\mathcal{D}_{i}$ come before all evidence sets in $\mathcal{D}_{j}$ for all $i<j$.

Depending on the amount of data available, $k<n$ is chosen as the number of blocks used for training. These blocks will be used to pick a promising candidate which should be evaluated on the testing data. In order to maximise the usage of the training data, we ignore the natural order of the data during training and use $k$-fold cross-validation. It should be noted that this is safe, since we only do this when choosing a promising candidate to evaluate, and do not use this scheme when evaluating the expected performance of the algorithm. Training then consists of holding out one of the $k$ blocks (known as the validation data), and estimating the parameters of the candidate using the rest of the blocks. This continues until every block in the training data has been held-out and validated upon. The performance score of the candidate is the average performance score on each held-out block.

Starting from index 1 , blocks $1, . ., k$ are used for training and block $k+1$ for testing, thus ensuring that the evidence sets in the testing data occurs after the training data (as in time series cross-validation, sometimes known as walk forward analysis [40] or rolling origin [55]). The procedure is then repeated starting from index 2 (i.e. blocks $2, . ., k+1$ are used for training and block $k+2$ for testing). By doing so we create repeated simulations, moving the testing data one block forward each time. An illustration of this procedure when $n=10$ and $k=3$ is show in Figure 3.6 .

\subsubsection{Algorithm}

Let $\mathcal{J}$ be a score function such that $\mathcal{J}\left(\mathcal{C}_{i}, \mathcal{D}_{j},\left\{\mathcal{D}_{l}, \ldots, \mathcal{D}_{m}\right\}\right)$ is the score for GBN candidate $\mathcal{C}_{i}$ (which is candidate $i$ of a GBN template, as defined in Section 3.2.1 when block $j$ has been used for either testing or validation and the blocks $\mathcal{D}_{l}, \ldots, \mathcal{D}_{m}$ have been used to estimate the parameters $\Theta$. In the case of template learning, $\Theta$ represents the parameters of the marginal and conditional probability distributions defined in the contained BNs. The algorithm then works in three steps (with an optional fourth): 


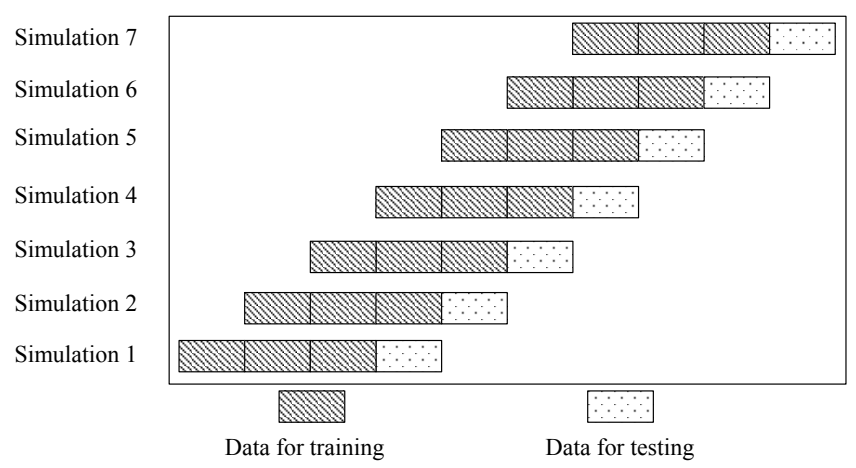

Figure 3.6: Multiple simulations used to evaluate the performance of a learning algorithm, using a total of $n=10$ blocks and $k=3$ blocks for training.

1. For each simulation $s$, where (as discussed previously) $\mathcal{D}_{s+k}$ is the testing data and $\mathcal{D}_{s}, \ldots, \mathcal{D}_{s+k-1}$ is the training data, find $\mathcal{C}^{s}$ that satisfies Equation 3.3. This corresponds to finding the GBN candidate with the maximum mean score of the $k$ evaluations performed during $k$-fold cross-validation over the training data. This is done by taking into consideration every possible candidate, thus exhausting the search space.

$$
\mathcal{C}^{s}=\underset{\mathcal{C}_{i}}{\arg \max } \frac{1}{k} \sum_{j=s}^{s+k-1} \mathcal{J}\left(\mathcal{C}_{i}, \mathcal{D}_{j},\left\{\mathcal{D}_{s}, \ldots, \mathcal{D}_{s+k-1}\right\} \backslash \mathcal{D}_{j}\right)
$$

2. For each $\mathcal{C}^{s}$ calculate its score $\rho_{\mathcal{J}}^{s}$ on the testing set with respect to the scoring function $\mathcal{J}$ according to Equation 3.4 This corresponds to estimating $\Theta$ of the found GBN candidate from Equation 3.3 using all training data and evaluating the performance on the data withheld for testing.

$$
\rho_{\mathcal{J}}^{s}=\mathcal{J}\left(\mathcal{C}^{s}, \mathcal{D}_{s+k},\left\{\mathcal{D}_{s}, \ldots, \mathcal{D}_{s+k-1}\right\}\right)
$$

3. The expected performance $\bar{\rho}_{\mathcal{J}}$ of the algorithm, with respect to the score function $\mathcal{J}$, is then given by the average of the scores $\rho_{\mathcal{J}}^{\mathcal{S}}$, as described in Equation 3.5 .

$$
\bar{\rho}_{\mathcal{J}}=\frac{1}{n-k} \sum_{s=1}^{n-k} \rho_{\mathcal{J}}^{s} .
$$

4. (Optional) If the objective is to find the candidate to be used on future data (i.e. block $\mathcal{D}_{n+1}$ ) then Equation 3.3 is used once more to find 
$\mathcal{C}^{n-k+1}$. This candidate can then be used on $\mathcal{D}_{n+1}$ with an expected performance $\bar{\rho}_{\mathcal{J}}$.

We emphasise that to ensure that each candidate is assessed on several blocks before selecting the one to move forward with, we allow that a validation block may come before some blocks used for parameter estimation in Equation 3.3. However, this step is only used to select a candidate to evaluate in Equation 3.4. The data used in Equation 3.4 is always ordered, i.e. the test block is always the immediate successor of the blocks used for parameter estimation.

In the description of the algorithm, one scoring function $\mathcal{J}$ has been used both for choosing a promising candidate in Equation 3.3 and for evaluating the expected performance of the algorithm in Equation 3.4. In Section 3.1.1 we have defined several metrics used to evaluate algorithmic trading systems. The scoring function $\mathcal{J}$ used in Equation 3.3 could internally use many of these metrics to come up with one score to compare the different candidates with. However, it is natural in the coming setting to expose the actual values of these metrics in Equation 3.4, and so several scoring functions $\mathcal{J}$ can be used to get a vector of scores $\left[\rho_{\mathcal{J}_{1}}^{s}, \ldots, \rho_{\mathcal{J}_{m}}^{s}\right]$ and use a vector of means as the performance of the algorithm $\left[\bar{\rho}_{\mathcal{J}_{1}}, \ldots, \bar{\rho}_{\mathcal{J}_{m}}\right]$.

\subsection{Learning gated Bayesian networks for trading stocks using template based learning}

We can now address our initial motivation for introducing GBNs, to use them as alpha models in algorithmic trading systems. We aim to use the template based learning algorithm to learn a GBN as an alpha model that generates buy and sell signals, such that the drawdown risks are mitigated as compared to the $\mathrm{BaH}$ strategy, while at the same time maintaining similar or better rewards.

\subsubsection{Methodology}

A GBN template with one BN per phase was created (see Figure 3.5), along with eight BNs per BN slot (see Figure 3.7) and four gates per gate slot, giving a total of 1024 candidates. The eight $\mathrm{BNs}$ used for $B N_{1}$ were identical to those used in $B N_{2}$, however the gates' trigger logic were different. The trigger logic for $G_{1}$ asks for the posterior probability of a good buying opportunity (i.e. a predicted positive future climate) while the trigger logic for 

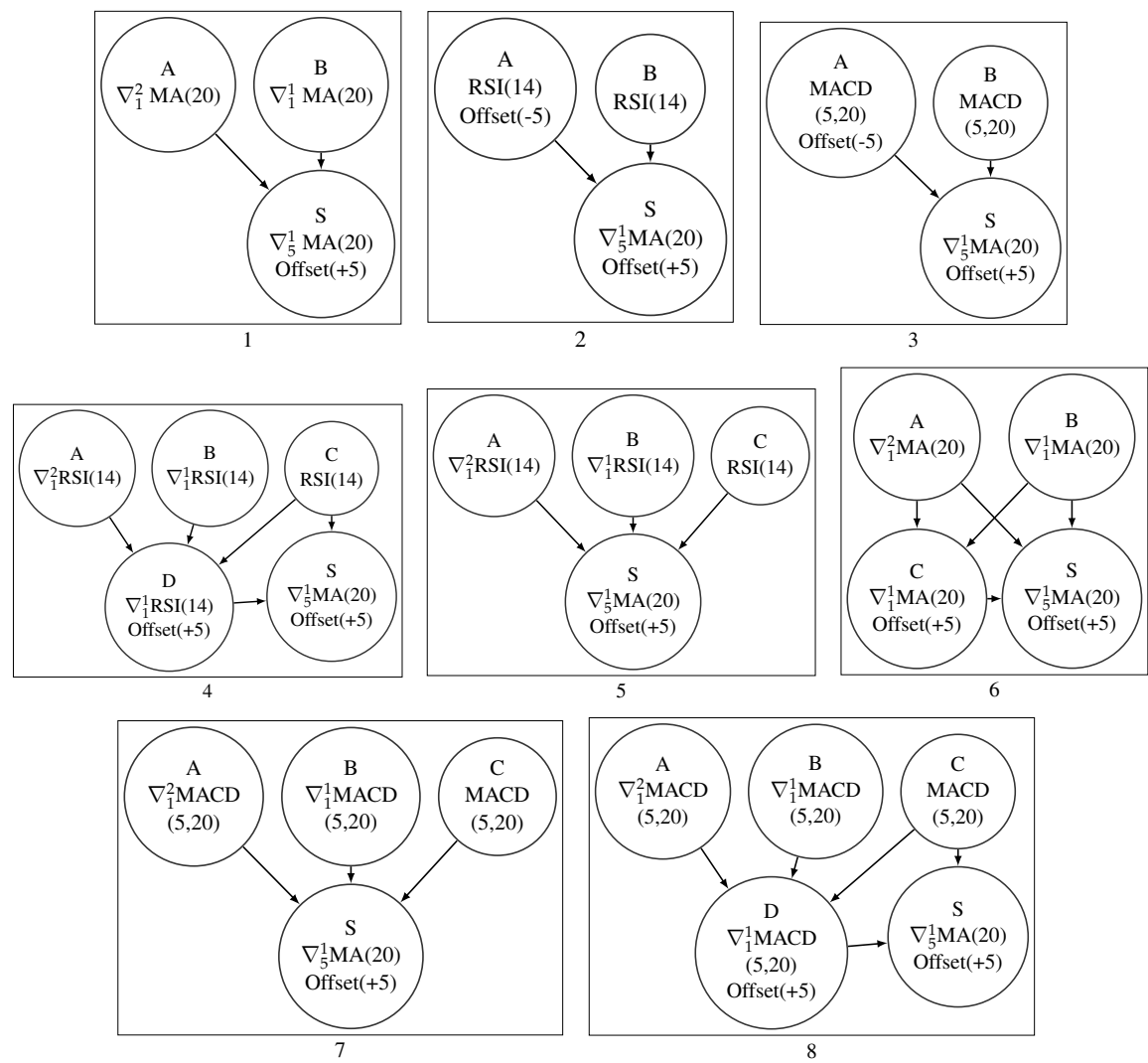

Figure 3.7: BNs in GBN template libraries.

$G_{2}$ asks for the posterior probability of a good selling opportunity (i.e. a predicted negative future climate). Each one of the four gates available for $G_{1}$ and $G_{2}$ had different thresholds which the posterior probability had to exceed in order for the gate to trigger (the thresholds were 0.5, 0.6, 0.7 and 0.8). The choice of variables and the structure of the eight BNs represent different experts' views on how to interpret the technical analysis indicators. For instance, network 2 in Figure 3.7 represents an expert that believes that RSI measured at its current value and its value five days in the past are indicative of future price movements, while network 3 believes the same but using the relative difference between two moving averages. These views are not exhaustive, however we assumed that these were the views producible by the available experts at the time of the application.

The random variables in the BNs were technical analysis indicators (MA, MACD and RSI) and their corresponding first and second order 1 and 5 day backward finite differences $\left(\nabla_{1}^{1}, \nabla_{5}^{1}, \nabla_{1}^{2}\right.$ and $\left.\nabla_{5}^{2}\right)$ which approximate the first 
and second order derivatives. The parameters used in the indicators are standard 14 day period for RSI [45] (written as RSI(14)), 20 day period for MA, representing 20 trading days in a month (written as MA(20)), and 5 and 20 day period for MACD, where 5 days represent the 5 trading days in a week (written as $\operatorname{MACD}(5,20)$ ). We also considered the previous indicators but with an offset of 5 days in the past and 5 days into the future. The random variables that were offset into the future represent the future economical climate, one of which was involved in the trigger logic of the gates. The true values for these future random variables were naturally not part of the testing data sets. Variables $A, B, C$ and $D$ were discretised into six bins, using the mean and one and two standard deviations above and below the mean as cutoff points. Variable $S$ was discretised into two bins, cutoff at zero so as to represent a positive and negative economical climate. The nodes representing variable $S$ in Figure 3.7 were used as trigger nodes for all gates. The GBN generated trading signals as it transitioned between its two phases.

\section{Data sets}

A set of actively traded stocks were chosen for the evaluation of the algorithm: Apple Inc. (AAPL), Amazon.com Inc. (AMZN), International Business Machines Corporation (IBM), Microsoft Corporation (MSFT), NVIDIA Corporation (NVDA), General Electric Company (GE) and Red Hat Inc. (RHT). The daily adjusted closing prices for these stocks between 2003-0101 and 2012-12-31 were downloaded from Yahoo! Finance ${ }^{\mathrm{TM}}$. This gave a total of 10 years of price data for each stock, where each year was allocated to a block, and thus $n=10$. For the learning algorithm, $k$ was chosen to be 3 , giving seven simulations from which to calculate $\left[\bar{\rho}_{\mathcal{J}_{1}}, \ldots, \bar{\rho}_{\mathcal{J}_{m}}\right]$. The split of the data is visualised in Figure 3.6 .

\section{Scoring functions}

The signals generated were backtested in order to calculate the relevant metrics. For step 1 in the learning algorithm (see Section 3.2.3 we used the Sharpe ratio. This choice was made as it combines both risk and reward into one score, which can then easily be compared between candidates. For step 2 we used the Sharpe ratio, return and drawdown risks described in Section 3.1 .1 to create a score vector. 

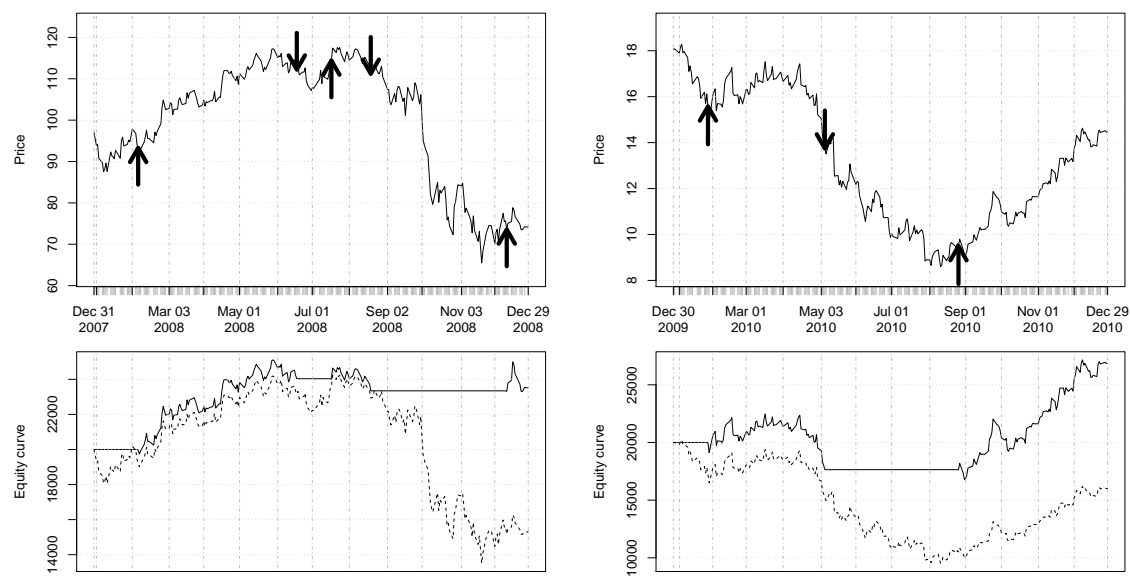

Figure 3.8: Price, signals and equity curve for IBM 2008 (left) and NVDA 2010 (right). The solid equity curve represents the GBN and the dashed equity curve represents $\mathrm{BaH}$.

\subsubsection{Results and discussion}

To visualise the backtesting that was done for each simulation, Figure 3.8 gives two examples of share price, generated signals (an upward arrow indicates a buy signal and a downward arrow indicates a sell signal) and resulting equity curve (with an initial investment of \$20000 USD) for the evaluated GBN. The solid line equity curve is the one achieved by executing the signals from the GBN, the dashed line is the corresponding equity curve for the $\mathrm{BaH}$ strategy. The GBN equity curve grows in a more monotonic fashion, which is desirable because this decreases the drawdown risks, while at the same time generating positive returns. The $\mathrm{BaH}$ strategy would have made a loss in both these examples, because the final price is lower than the initial one, furthermore it would have displayed bad intermediate behaviour, reflected by the high drawdown risk values that would have been incurred. These are declining years for the share prices, however the GBN does its best to get as much value as possible from the price movements.

Table 3.1 presents the score vectors from the learning algorithm versus the score vector of the BaH strategy over the seven simulations. Rows named min, max and $s d$ (standard deviation) are based on Equation 3.4, while mean corresponds to Equation 3.5. As each block used by the learning algorithm had an approximate length of one year, the Sharpe ratio that is given by dividing the mean with the sd of the return column is a yearly Sharpe ratio based on seven years (where the risk-free rate has not been included). The acronyms MDD, MDDD, LVFI and TIMR, represent the metrics described 
in Section 3.1.1. All values are ratios except for MDDD which is measured in number of days.

\section{Analysis of results}

The Sharpe ratio is our measure of reward, premiered above the raw return for reasons discussed in Section 3.1.1. Our first concern is to ensure that the learnt GBNs are producing similar or better Sharpe ratios than the $\mathrm{BaH}$ strategy over the testing period. As can be seen in Table 3.1, this is the case except for NVDA and RHT. As we have previously discussed, it requires a very high accuracy of predictions to consistently beat the Sharpe ratio of $\mathrm{BaH}$.

From this we can conclude that the GBNs do not get beaten consistently by the BaH strategy when considering the annual Sharpe ratio, even though it is considered a nearly optimal strategy. Furthermore, we should take into consideration TIMR. The GBNs are spending less time in the market, reducing risk to equity and possibly increasing equity value from risk free investments. Potential gain in equity from risk free rates have not been added to the Sharpe ratios presented in the table. Considering that the learnt GBNs consistently spend considerably less time in the market (shown by the low TIMR values), this could give a significant boost to the Sharpe ratios. An example of this can be seen for NVDA where the Sharpe ratio for GBN is lower than for $\mathrm{BaH}$, but the GBN only spent on average $51.6 \%$ of the time in the market, risk free investments could potentially drive the Sharpe ratio for the GBN above that of the BaH strategy.

Turning our attention to the drawdown risks, we first consider the MDD and MDDD. The difference of the MDD values are substantial, the MDD mean and sd are consistently smaller for the GBNs than they are for the BaH strategy. This signals that the equity we gain from our investments are at less risk when using the GBNs compared to the BaH strategy. For MDDD the GBNs outperform the BaH strategy in five out of seven cases.

The LVFI is a major threat to equity (see Section 3.1.1), and it is the one metric where $\mathrm{BaH}$ severely underperforms. Considering the max values we note that for NVDA the BaH strategy wiped out $82.1 \%$ of the equity at worst, while the GBNs did $46.7 \%$ at worst for NVDA. Considering the LVFI mean and sd for all stocks we note that they are consistently almost half for the GBNs compared to the BaH strategy. LVFI is important because it is the risk of the initial investment, losing much of the initial investment may lead to premature withdrawal of funds and/or force liquidation by margin-calls. 


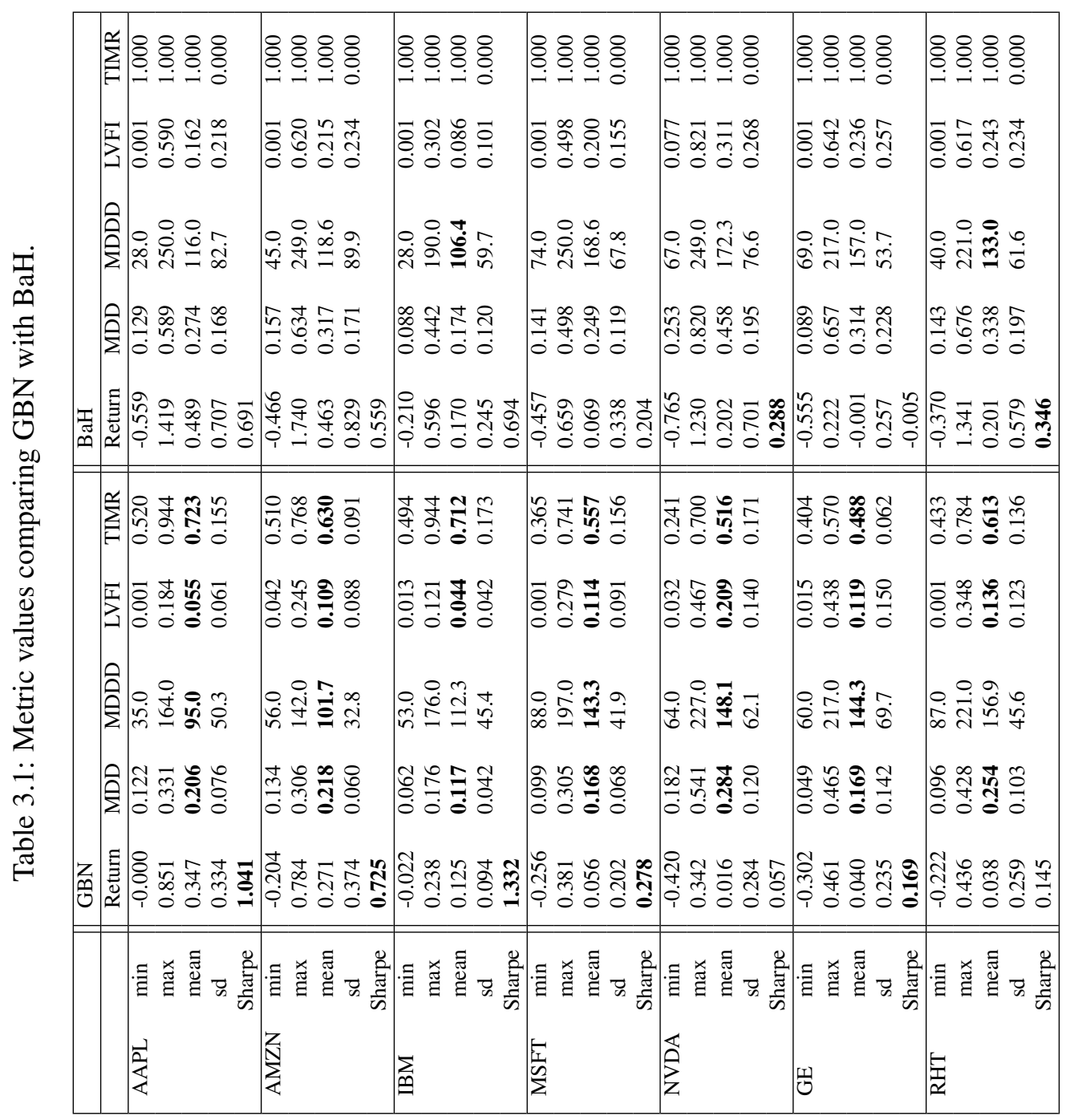


Table 3.2: Annual Sharpe ratio for single BN and GBN.

\begin{tabular}{|l||lllllll|}
\hline & AAPL & AMZN & IBM & MSFT & NVDA & GE & RHT \\
\hline Single BN & 0.675 & 0.561 & 0.440 & 0.018 & 0.043 & 0.142 & 0.120 \\
GBN & $\mathbf{1 . 0 4 1}$ & $\mathbf{0 . 7 2 5}$ & $\mathbf{1 . 3 3 2}$ & $\mathbf{0 . 2 7 8}$ & $\mathbf{0 . 0 5 7}$ & $\mathbf{0 . 1 6 9}$ & $\mathbf{0 . 1 4 5}$ \\
\hline
\end{tabular}

All in all, the results clearly indicate that GBNs are competitive with $\mathrm{BaH}$ in terms of Sharpe ratio, whereas they induce a more desirable behaviour in terms of MDD, LVFI and TIMR.

\section{Single Bayesian network comparison}

We have made a leap into immediately assuming that having different BNs for the different phases in the template pictured in Figure 3.5 would be an improvement above having the same BN in both phases. This assumption stems from the underlying hypothesis of GBNs, that different BNs are required at different phases of a process. However, in order to illuminate upon the difference between using the same BN for each phase compared to our results in Table 3.1, we ran the same experiment again, however this time only using the subset of candidate GBNs that had the same BN in both phases. For brevity we only report the comparison of annual Sharpe ratios in Table 3.2. As is evident, GBNs with different BNs in the different phases outperform the single BN for all stocks. For some stocks the difference is marginal (NVDA, GE and RHT), while for others the difference is substantial (AAPL, AMZN, IBM and MSFT).

From this we conclude that there is evidence for the underlying hypothesis of GBNs, that different BNs are required at different phases of a process. Without having further investigated the origins of this improvement, it does seem to suggest that buying opportunities and selling opportunities are not each others counterparts. Note that the $\mathrm{BaH}$ strategy in general outperformed the single BN approach with respect to the annual Sharpe ratio.

\section{Post-analysis}

One of the benefits of using BNs is that we can get transparency as to why a particular signal was generated. Our aim here was to look at the nondiscretised values of the variables at the time a signal was generated. We combined the signals from all simulations (regardless of which stock was traded) and then grouped the signals by which BN generated them and if they were buy or sell signals. We then did pair-wise combinations of the variables in each $\mathrm{BN}$ to create scatter plots with values of the variables along 

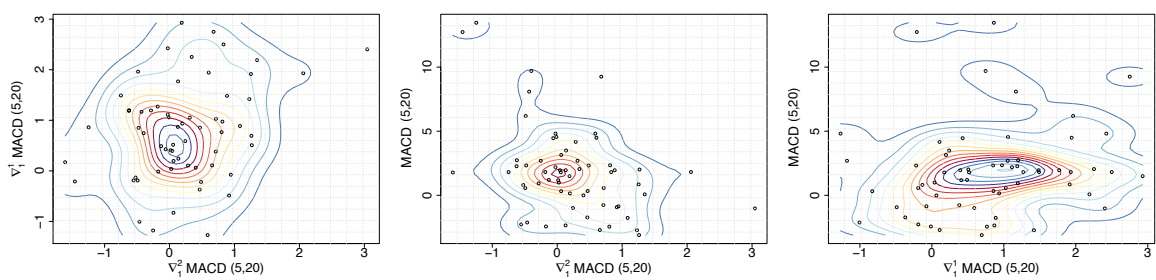

(a)
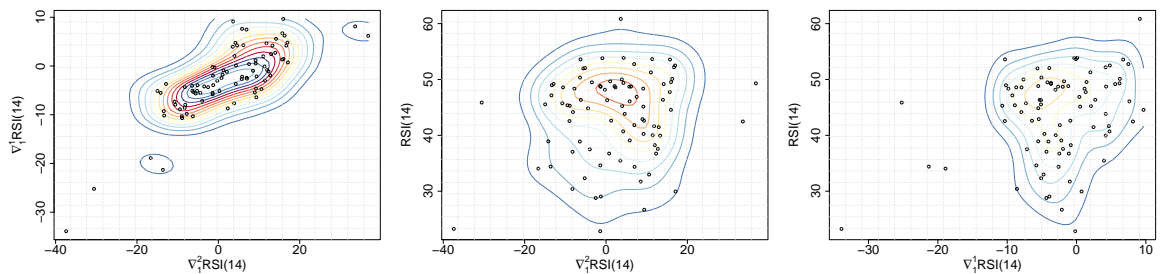

(b)

Figure 3.9: In (a), buy decisions using network 7 from Figure 3.7 , In (b), sell decisions using network 5 from Figure 3.7

the axes and also added an approximated density using the frequency of signals. These scatter plots show when GBNs are generating signals. Examples of these plots for the BNs that generated the most signals are given in Figure 3.9a (using network 7 from Figure 3.7) and Figure 3.9b (using network 5 from Figure 3.7).

In Figure 3.9a the $\mathrm{BN}$ is used to look for buying opportunities. In the first plot we see that most signals are generated when both $\nabla_{1}^{1} \operatorname{MACD}(5,20)$ and $\nabla_{1}^{2} M A C D(5,20)$ are positive, indicating that the difference between the two MAs is growing and increasing in speed, but not so positive so as to making it impossible to benefit from the trend. The second two plots in Figure 3.9a plot $\nabla_{1}^{2} M A C D(5,20)$ against $\operatorname{MACD}(5,20)$ and $\nabla_{1}^{1} \operatorname{MACD}(5,20)$ against $M A C D(5,20)$. Both these confirm what we knew about the first and second order difference, but also indicate that $\operatorname{MACD}(5,20)$ should be positive (so the fast period MA should be above the slow period MA). From a technical analysis perspective this type of pattern is common, it indicates a trend change, as the fast MA is moving above and away from the slow MA. It is noteworthy to mention that we have not set any priors on the BNs that would indicate that these are the kind of patterns we are interested in, so our learning algorithm is able to re-discover these human-like commonly used patterns. An example of selling signals is presented in Figure 3.9b, here we are using RSI which is bounded between 0 and 100. When RSI moves up to- 
wards 100 it indicates that the buying pressure is increasing, and should drive prices higher, the opposite is true when RSI moves towards 0 . The first plot indicates that most selling signals are generated when $\nabla_{1}^{1} R S I(14)$ is close to zero or negative (i.e. RSI has started to decrease) and $\nabla_{1}^{2} R S I(14)$ is bounded around \pm 10 . The two other plots in Figure $3.9 \mathrm{~b}$ represent $\nabla_{1}^{2} R S I(14)$ against $R S I(14)$ and $\nabla_{1}^{1} R S I(14)$ against $R S I(14)$. These last two figures confirm our findings in the first figure, but also indicates that the RSI(14) should be below 50 (but not too much below 50 so as to miss the selling opportunity). This seems reasonable from a technical analysis perspective, as RSI goes below 50 and decreases, the selling pressure increases, indicating that the price will go lower, and so a selling signal is generated. We reemphasise that we did not set any prior in the BNs that would suggest that these are the type of signals we should be looking for.

\subsubsection{Extended experiments}

In the main experiments we used the posterior probability of a variable being in a specific state as input to the trigger logic of the gates. Thus the trigger logic of the gates were not directly based on the Sharpe ratio, which is the score we ultimately wish to maximise. Since we did not beat the Sharpe ratio of BaH for NVDA and RHT, we wanted to attempt to improve these results. According to Definition 1 and Definition 4 we can introduce utility nodes into the BNs and use them as trigger nodes. By doing so, we aimed at instructing the candidates to behave in such a way that they premiered the Sharpe ratio.

The Sharpe ratio itself is defined as the mean of a set of returns divided by the standard deviation of those returns (see Section 3.1.1. At the time the trigger logic for a gate is evaluated, the information available is the posterior weighted utilities given some evidence. Thus, it is not possible to compute the Sharpe ratio for a specific data point, but rather the expected risk adjusted return. Given a discrete variable $S$ that represents the future return of an investment over some period of time, we can construct a logical statement regarding the expected risk adjusted return according to Equation 3.6. This 
is a reasonable approach for instructing the candidates to premier the Sharpe ratio.

$$
\begin{aligned}
T L(\cdot) & : \frac{\mu_{t}}{\sqrt{\sum_{i}\left(u(S=i)-\mu_{t}\right)^{2} p\left(S=i \mid d_{t}\right)}}>\tau \\
\mu_{t} & =\sum_{i} p\left(S=i \mid d_{t}\right) u(S=i)
\end{aligned}
$$

$p\left(S=i \mid d_{t}\right)$ probability that $S$ is in state $i$ given data point $d_{t}$

$u(S=i)$ utility of $S$ when it is in state $i$

All trigger nodes ( $S$ in Figure 3.7) represent the first order 5 day backward finite difference of the 20 day MA of the share price, offset 5 days into the future. These trigger nodes were replaced with the 5 day return of the 20 day MA, offset 5 days into the future. We kept the 20 day MA as a smoother in order to make as few changes as possible between the two experiments. These new nodes were discretised using six bins 1 and a utility node was added that mapped each one of these bins to the mean value of the content of the bin (the candidates looked similar to Figure 2.6b. The trigger logic for the gates were set according to Equation 3.6. This implies that each time a GBN was presented with new data, it calculated an expected risk adjusted return based on the current posterior distribution of the variable $S$, and if this was higher or lower than some threshold $\tau$ then it triggered, generating a signal.

The results of the extended experiment are shown in Table 3.3 . For compactness we only present the mean values of each score and the annual Sharpe ratio. For AAPL and AMZN we did not improve upon the results from the main experiments. For IBM, MSFT, NVDA, GE and RHT we were able to improve the Sharpe ratio. For NVDA the improvement was clear, but not enough to beat $\mathrm{BaH}$, on the other hand for RHT we were able to improve the Sharpe ratio enough to beat $\mathrm{BaH}$. It does however seem to come at a cost, as the other score metrics were not necessarily improved upon over all stocks. This is most likely due to the fact that we prioritised the Sharpe ratio by using the expected risk adjusted return in the trigger logic.

\subsection{Learning using Bayesian optimisation}

In Section 3.3 we saw that the template based approach to learning GBNs was successful at learning alpha models that were competitive with the BaH strategy. There are however a few requirements of the template based approach

\footnotetext{
${ }^{1}$ Using the mean and one and two standard deviations above and below the mean as cutoff points.
} 
Table 3.3: Metric values comparing GBN, GBN with utility and BaH.

\begin{tabular}{|ll||lllll|}
\hline & & Sharpe & MDD & MDDD & LVFI & TIMR \\
\hline AAPL & GBN & $\mathbf{1 . 0 4 1}$ & $\mathbf{0 . 2 0 6}$ & $\mathbf{9 5 . 0}$ & $\mathbf{0 . 0 5 5}$ & $\mathbf{0 . 7 2 3}$ \\
& GBN with utility & 0.821 & 0.236 & 105.0 & 0.086 & 0.807 \\
& BaH & 0.691 & 0.274 & 116.0 & 0.162 & 1.000 \\
\hline AMZN & GBN & $\mathbf{0 . 7 2 5}$ & $\mathbf{0 . 2 1 8}$ & $\mathbf{1 0 1 . 7}$ & $\mathbf{0 . 1 0 9}$ & $\mathbf{0 . 6 3 0}$ \\
& GBN with utility & 0.469 & 0.292 & 117.0 & 0.183 & 0.692 \\
& BaH & 0.559 & 0.317 & 118.6 & 0.215 & 1.000 \\
\hline IBM & GBN & 1.332 & 0.117 & 112.3 & $\mathbf{0 . 0 4 4}$ & $\mathbf{0 . 7 1 2}$ \\
& GBN with utility & $\mathbf{1 . 4 4 0}$ & $\mathbf{0 . 1 1 2}$ & 108.0 & 0.054 & 0.713 \\
& BaH & 0.694 & 0.174 & $\mathbf{1 0 6 . 4}$ & 0.086 & 1.000 \\
\hline MSFT & GBN & 0.278 & 0.168 & 143.3 & 0.114 & $\mathbf{0 . 5 5 7}$ \\
& GBN with utility & $\mathbf{0 . 3 7 6}$ & $\mathbf{0 . 1 6 2}$ & $\mathbf{1 4 3 . 0}$ & $\mathbf{0 . 0 9 9}$ & 0.572 \\
& BaH & 0.204 & 0.249 & 168.6 & 0.200 & 1.000 \\
\hline NVDA & GBN & 0.057 & $\mathbf{0 . 2 8 4}$ & $\mathbf{1 4 8 . 1}$ & $\mathbf{0 . 2 0 9}$ & $\mathbf{0 . 5 1 6}$ \\
& GBN with utility & 0.216 & 0.313 & 149.0 & 0.220 & 0.518 \\
& BaH & $\mathbf{0 . 2 8 8}$ & 0.458 & 172.3 & 0.311 & 1.000 \\
\hline GE & GBN & 0.169 & 0.169 & 144.3 & 0.119 & $\mathbf{0 . 4 8 8}$ \\
& GBN with utility & $\mathbf{0 . 4 1 6}$ & $\mathbf{0 . 1 2 9}$ & $\mathbf{1 3 7 . 0}$ & $\mathbf{0 . 0 7 2}$ & 0.510 \\
& BaH & -0.005 & 0.314 & 157.0 & 0.236 & 1.000 \\
\hline RHT & GBN & 0.145 & $\mathbf{0 . 2 5 4}$ & 156.9 & $\mathbf{0 . 1 3 6}$ & $\mathbf{0 . 6 1 3}$ \\
& GBN with utility & $\mathbf{0 . 4 8 5}$ & 0.257 & 153.0 & 0.153 & 0.734 \\
& BaH & 0.346 & 0.338 & $\mathbf{1 3 3 . 0}$ & 0.243 & 1.000 \\
\hline
\end{tabular}

that we wish to address, namely the reliance on prior expert knowledge regarding the BN structures, and the exhaustive search over GBN candidates. The first requirement limits the applicability of the algorithm to areas where prior knowledge is available, while the second requirement limits the number of phases and transitions that can be modelled, as the number of GBN candidates may become too many to evaluate.

Reducing our reliance on prior expert knowledge requires us to search a much larger space of BN structures, which may lead to an enormous search space over which exhaustive search would not be feasible. Furthermore, if we were to introduce more phases in our GBN templates we would generate even more candidates. A potential approach for reducing the search space is to use naïve Bayes classifiers (NBCs) rather than full unrestricted BNs. The NBC models the variables such that they are independent given a class variable, therefore the structure is fixed and does not require prior expert knowledge (generally at the cost of no longer having a model that represents the generative process). Furthermore, we would prefer if we could automate (at least to some degree) the selection of variables that we place in the NBCs.

With the desire to both select which variables that we place in our NBCs, and extend the number of modelled phases and transitions, the exhaustive search required by the template based approach becomes infeasible. We therefore need to employ some optimisation scheme that allows us to (hopefully) find the best candidate without having to evaluate all of them. Bayesian 
optimisation [56] has become a popular technique for global optimisation of expensive black box functions, and in our case maximising the Sharpe ratio via GBNs is a sort of expensive black box function, thus we shall employ this technique in order to make the candidate search feasible.

To clarify, we shall employ the same technique for multiple simulations that we presented in Section 3.2.2, and the same equations for performance evaluation as in Section 3.2.3. However, rather than finding the GBN candidate that maximises Equation 3.3 by exhaustive search, we shall employ Bayesian optimisation to find the maximum. Furthermore, we shall employ a different scheme for GBN candidate generation.

In Section 3.4.2 we shall discuss the details of the Bayesian optimisation procedure that we employ to maximise Equation 3.3. Before doing so however, we shall discuss how the GBN candidates are generated, and in order to avoid making this discussion more abstract than necessary, we shall partially introduce the real-world application that we shall use to evaluate the algorithm, thereby allowing a mental image of the intended use of the candidate generation while we introduce it.

\subsubsection{Using naïve Bayes classifiers within gated Bayesian networks for algorithmic trading}

Rather than using a library of BNs, as was the case in the template based approach, we shall now create NBCs which use technical analysis indicators as random variables. We begin by using the same GBN template as the one depicted in Figure 3.5, and then place a NBC in each of the BN slots (i.e. where it says $B N_{1}$ and $B N_{2}$ ). The resulting GBN is depicted in Figure 3.10 , where the two gates $G_{1}$ and $G_{2}$ have a parameter ( $\tau_{1}$ and $\tau_{2}$ respectively) that decides the threshold in their trigger logic.

Apart from $\tau_{1}$ and $\tau_{2}$, we also use a parameter in the buy phase $\left(\psi_{b u y}\right)$ and a parameter in the sell phase $\left(\psi_{\text {sell }}\right)$ which decide one of the parameters of the technical analysis indicators. This setup is a first step in becoming less reliant upon expert knowledge, as we shall optimise the choice of these four parameters $\left(\tau_{1}, \tau_{2}, \psi_{\text {buy }}\right.$ and $\left.\psi_{\text {sell }}\right)$. To clarify, choosing a value for each of the parameters $\tau_{1}, \tau_{2}, \psi_{b u y}$ and $\psi_{\text {sell }}$ creates a GBN candidate, and allowing $\psi_{b u y}$ to be different from $\psi_{\text {sell }}$ allows the model to use different variables for the different phases (e.g. using a 20 day MA period for buy and 10 day MA period for sell creates different variables in the different NBCs). Thus the NBCs that we use all have the same structure, one class variable with four children, however we allow the $\psi$ values to differ. 


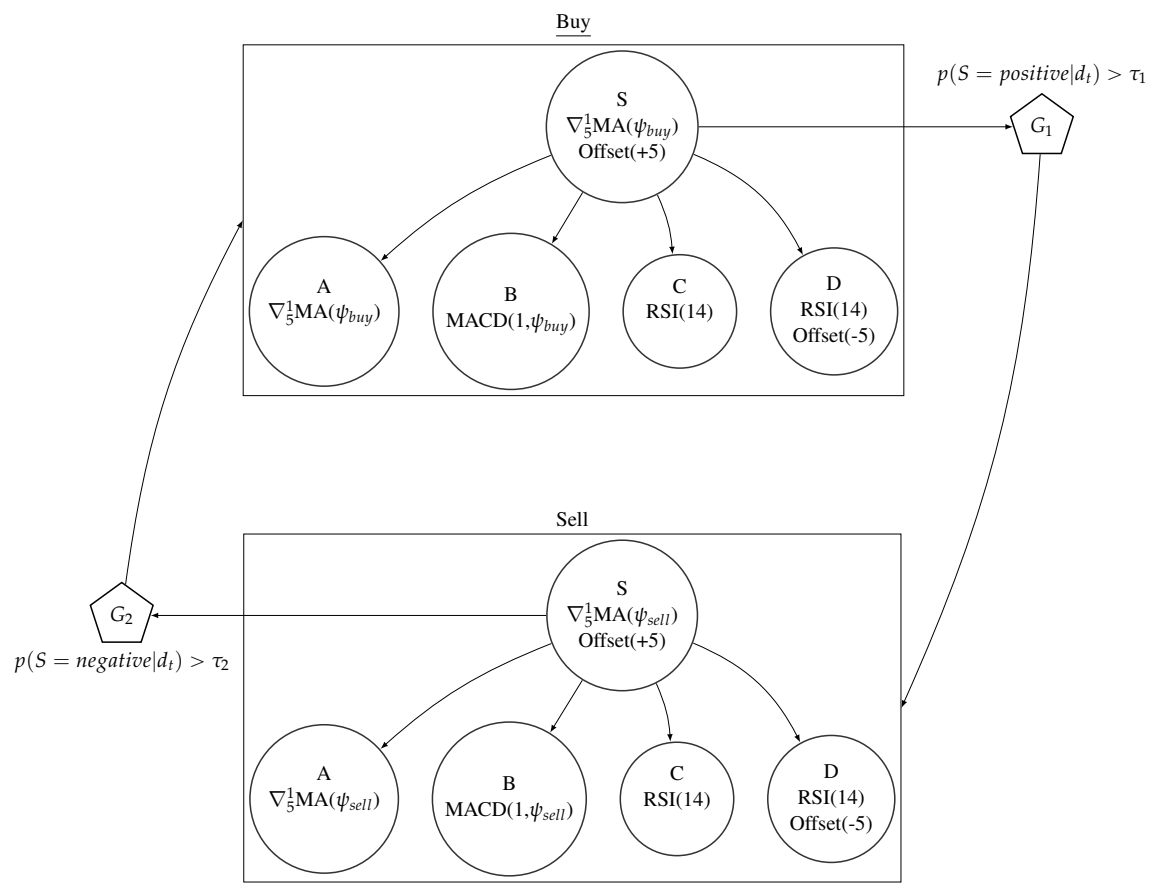

Figure 3.10: GBN using NBCs in the different phases for buy and sell.

By doing so we can generate GBN candidates (much like the template approach), and the remaining task is then to pick the best GBN candidate. Since we are now working in a much larger search space (the parameters allow for an infinite number of candidates), we cannot use the exhaustive approach used previously. We therefore now turn our attention to Bayesian optimisation in an attempt to solve this remaining task.

\subsubsection{Gaussian processes and Bayesian optimisation}

From Section 3.4.1 it is clear that there are parameters that we wish to search over such that we find the best candidate. We shall in general refer to the parameters that we wish to search over as $\Lambda$. We recall from Section 3.2 .2 that, for each simulation, we must first decide upon the parameters $\Lambda$ that optimise the cross-validation score over the training data, and then use these parameters on the held out test data. We let $f(\Lambda)$ represent this cross-validation score, where we have for brevity removed the training data from the notation, keeping in mind that $f$ operates over some given data. 


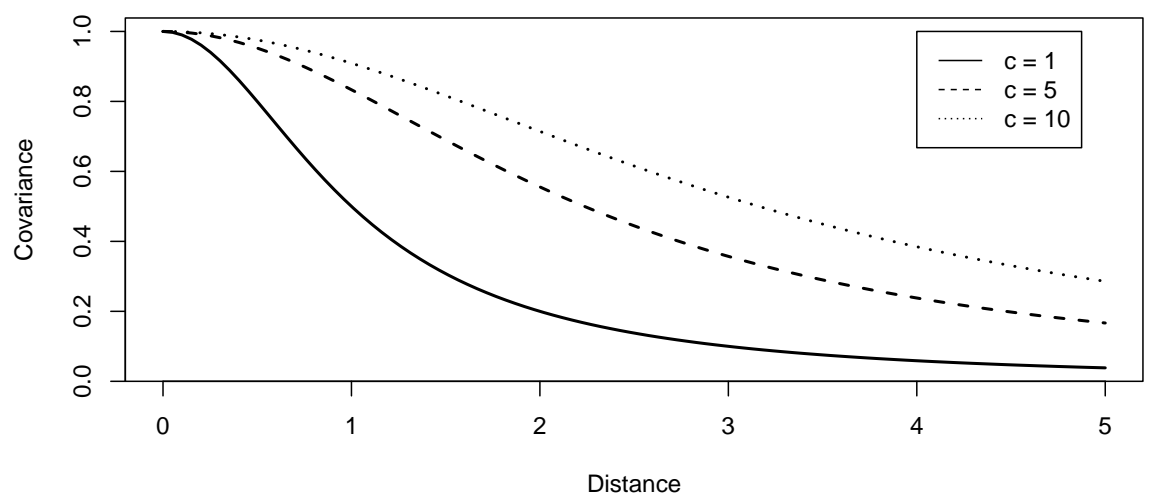

Figure 3.11: Covariance decreases by distance.

While we can evaluate $f$ at a given point $\Lambda$, we do not have a closed form expression for $f$, thus we cannot solve the problem analytically. Instead, we will introduce a random variable $g$ to act as a proxy for $f$, and place on $g$ a prior over all possible functions. This is done by using a Gaussian process (GP), which is defined as a distribution over an infinite number of variables, such that any finite subset of these variables are jointly multivariate Gaussian. Thus, for each $\Lambda$ we treat $g(\Lambda)$ as a Gaussian random variable, where $f(\Lambda)$ is a realisation of the random variable $g(\Lambda)$, and treat a set of such random variables as jointly multivariate Gaussian.

Formally, the GP is parameterised by a mean function $\mu(\Lambda)$, and a covariance kernel $k\left(\Lambda, \Lambda^{\prime}\right)$, which are defined over all input pairs $\Lambda, \Lambda^{\prime} \in \Lambda$. The mean function $\mu(\Lambda)$ represents the expected value of $g(\Lambda)$, and it is commonly assumed to be zero for all $\Lambda \in \Lambda$, although this is not necessary if prior information exists to suggest otherwise. The covariance kernel $k\left(\Lambda, \Lambda^{\prime}\right)$ represents how much the random variables $g(\Lambda)$ and $g\left(\Lambda^{\prime}\right)$ covary, thus it defines how smooth the function $f$ is thought to be, and can therefore be tuned to one's prior belief about $f$. For instance, by using the rational quadratic kernel in Equation 3.7, we can tune $c$ to fit our prior belief about smoothness.

$$
k\left(\Lambda, \Lambda^{\prime}\right)=1-\frac{\left\|\Lambda-\Lambda^{\prime}\right\|^{2}}{\left\|\Lambda-\Lambda^{\prime}\right\|^{2}+c}
$$

For points close to each other, Equation 3.7 will result in values close to 1 , while points further away will be given values closer to 0 . The GP prior will obtain the same smoothness properties, as the covariance matrix 
is completely defined by $k$. To visualise the smoothness achieved by tuning $c$, Figure 3.11 shows the decreasing covariance as distance grows with three different settings of $c(1,5$ and 10). As can be seen, as $c$ increases the decrease is slower, thus more smoothness is assumed.

Let $\Lambda_{1: i}$ represent $i$ different $\Lambda \mathrm{s}$, without any specific ordering, and let $f_{1: i}$ represent the value of $f$ at these $\Lambda$ s, i.e. $f_{j}=f\left(\Lambda_{j}\right)$. Similarly, let $g_{j}$ represent the random variable $g\left(\Lambda_{j}\right)$. Having collected observations $\left\{\Lambda_{1: i}, f_{1: i}\right\}$, we can calculate the posterior distribution of $g$ for some new input $\Lambda_{i+1}$, where both the prior smoothness and the observed data have been considered. A closed form expression exists for this calculation as described in Equation 3.8. Notice that Equation 3.8 is the usual equation to compute the conditional density function of $X$ given an observation for $Y$, when $X$ and $Y$ are jointly Gaussian. For more on GPs, please see [57].

$$
\begin{aligned}
& {\left[\begin{array}{c}
g_{1: i} \\
g_{i+1}
\end{array}\right] \sim \mathcal{N}\left(\mathbf{0},\left[\begin{array}{cc}
\mathbf{K} & \mathbf{K}_{*}^{\mathbf{T}} \\
\mathbf{K}_{*} & \mathbf{K}_{* *}
\end{array}\right]\right)} \\
& \mathbf{K}=\left[\begin{array}{ccc}
k\left(\Lambda_{1}, \Lambda_{1}\right) & \cdots & k\left(\Lambda_{1}, \Lambda_{i}\right) \\
\vdots & \ddots & \vdots \\
k\left(\Lambda_{i}, \Lambda_{1}\right) & \cdots & k\left(\Lambda_{i}, \Lambda_{i}\right)
\end{array}\right] \\
& \mathbf{K}_{*}=\left[\begin{array}{lll}
k\left(\Lambda_{i+1}, \Lambda_{1}\right) & \cdots & k\left(\Lambda_{i+1}, \Lambda_{i}\right)
\end{array}\right] \\
& \mathbf{K}_{* *}=k\left(\Lambda_{i+1}, \Lambda_{i+1}\right) \\
& p\left(g_{i+1} \mid\left\{\Lambda_{1: i}, f_{1: i}\right\}\right)=\mathcal{N}\left(\mu_{i}\left(\Lambda_{i+1}\right), \sigma_{i}^{2}\left(\Lambda_{i+1}\right)\right) \\
& \mu_{i}\left(\Lambda_{i+1}\right)=\mathbf{K}_{*} \mathbf{K}^{-1} f_{1: i} \\
& \sigma_{i}^{2}\left(\Lambda_{i+1}\right)=\mathbf{K}_{* *}-\mathbf{K}_{*} \mathbf{K}^{-1} \mathbf{K}_{*}^{T}
\end{aligned}
$$

Since $g$ is acting as a proxy for the objective function $f$, using a GP allows us to encode prior beliefs about the objective function, and sampling the objective function allows us to update the posterior over objective functions. In the framework of Bayesian optimisation, we utilise the posterior over objective functions to inform us of how to iterate between sampling and updating, in order to find the $\Lambda$ that maximises the objective function $f$. The next $\Lambda$ for which to evaluate $f$ is the $\Lambda$ that maximises an acquisition function. Several acquisition functions have been suggested, however the goal is to trade off exploring areas where the posterior uncertainty is high, while exploiting points that have a high posterior mean. We will use the upper confidence bound criterion, which is expressed as $U C B(\Lambda)=\mu(\Lambda)+\eta \sigma(\Lambda)$, where $\mu(\Lambda)$ and $\sigma(\Lambda)$ represent the posterior mean and standard deviation of $g(\Lambda)$, 
and $\eta$ is a tuning parameter to allow for more exploration (as $\eta$ is increased) or more exploitation (as $\eta$ is decreased). Once a predetermined number of iterations $m$ have passed, the $\Lambda_{i}$ for which $f_{i}$ is greatest in $\left\{\Lambda_{1: m}, f_{1: m}\right\}$ is the set of parameters that maximises the objective function.

\subsubsection{Learning algorithm}

We have so far given the moving parts of the proposed learning algorithm, and we shall here just briefly summarise the entire process:

1. Create the structure of the GBN, i.e. decide upon the phases that should be modelled. Place a NBC within each one of these phases, which in turn have certain parameters that allow for feature selection (in the case considered here, we adjust the parameters of the technical analysis indicators to create different features).

2. Split the available data and create multiple simulations (as in Section 3.2.2.

3. For each simulation, use Bayesian optimisation to decide upon the feature selection parameters and the thresholds within the gates' trigger logic that has the highest average cross-validation score over the training data, and then use these parameters on the held out test data.

4. Calculate the expected performance of the algorithm according to Equation 3.5 .

To show how this algorithm can be used in a real-world context, we shall in Section 3.5 return to the algorithmic trading scenario. This time we shall consider scenarios with both two and three phases, to show how the proposed algorithm can deal with the increased number of phases, which would become difficult for the template based approach.

\subsection{Learning gated Bayesian networks for index trading using Bayesian optimisation learning}

Stock indices are weighted averages of their respective stock components. For instance, the Dow Jones Industrial Average (DJIA) is a weighted average of 30 large companies based in the United States. Indices may have different schemes for how the different components are weighted, however they all aim to give a collective representation of their components. 
An index fund owns shares of the components of a specific index, proportional to the weights, such that the fund's return is mirrored by the index. These funds are very popular, as they are easy for the investor to comprehend but at the same time trading the individual components of an index requires a lot of effort.

A BaH strategy on stock indices via index funds may be convenient, however it implies that the equity is put through the full force of drawdown risks described in Section 3.1.1. In dwindling markets, the index fund will lose value, and equity could be salvaged and possibly be placed in risk-free assets during these periods. Furthermore, utilising certain financial products, it is also possible to increase equity during these times of distress by purchasing short positions of the index [58]. When purchasing a short position an investor borrows assets from a second party and then immediately sells them. At a later point in time the investor may buy the same assets and give them back to the lender. If the price was lower when the investor bought the assets then a profit is made from the assets decreasing in value. To make the distinction, regular positions are called long when short positions are in the context considered. We chose to run our experiments in this section using indices for two reasons: first, the popularity of passive index funds is undeniable, thus it is a relevant benchmark to beat, and second, it is sometimes not possible to short sell single stocks due to regulations (e.g. the Securities and Exchange Commission placed a ban on short selling of 799 stocks during the height of the financial crisis in 2008).

We shall account for two sets of experiments, where in the first we shall employ a GBN that models buying and selling stock indices, and in the second we shall incorporate the possibility of taking short positions.

\subsubsection{Methodology}

For the two sets of experiments we used two different GBN templates to generate candidates. The first GBN template (henceforth known as GBN-1) modelled buying and selling long positions only, while the second GBN template (GBN-2) modelled buying and selling long and short positions. Template GBN-1 is depicted in Figure 3.10 and GBN-2 in Figure 3.12.

How buy and sell signals are generated when using GBN-1 has been described previously (see Section 2.2.4 and Section 3.3.1. Template GBN-2 starts in the Trend phase, from where either $G_{1}$ or $G_{2}$ can trigger. The Trend phase predicts the future economical climate, and if there is a large enough probability of a positive or negative climate a phase change is made (if there is not a large enough probability for a move in either direction the model 


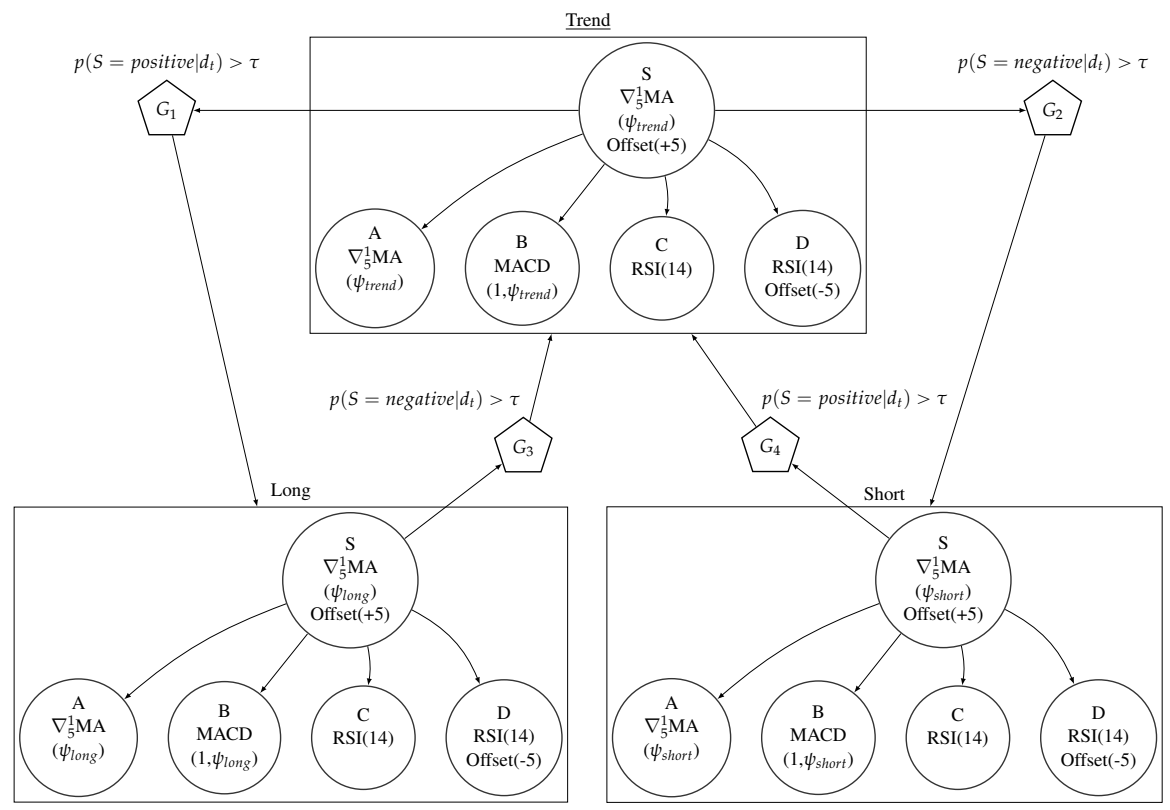

Figure 3.12: GBN using NBCs in the different phases for buying and selling both long and short positions.

stays in this Trend phase). If $G_{1}$ triggers then a long open signal is generated and the Long phase is activated (deactivating the Trend phase and indicating to the investor to buy the index). If then gate $G_{3}$ triggers then a long close signal is generated, and the Trend phase is activated again (deactivating the Long phase and indicating to the investor to sell the index previously bought). However, if before $G_{1}$ triggers $G_{2}$ triggers instead, then a short open signal is generated, and the Short phase is activated (deactivating Trend and indicating to the investor to buy a short position in the index). In similar fashion, when $G_{4}$ triggers a short close signal is generated: activating Trend, deactivating Short and indicating that the short position should be sold.

\section{Variables}

The variables used in the NBCs were as before based on technical analysis indicators. Because we were attempting to reduce the reliance on prior expert knowledge we attempted to set some parameters to prevailing standards (e.g. 14 periods for RSI [45, 59]), and the choice of 5 periods as the prediction horizon was based on the number of trading days in a week. Instead of attempting to choose a fast period for the MACD indicator, and to avoid 
optimising it, we set it to 1 (i.e. no averaging at all, so the indicator becomes the relative difference between the raw price and a MA).

Variables $A, B, C$ and $D$ were discretised into six bins, each using equal width binning ${ }^{2}$ and $S$ was discretised into two bins separated by zero. Thus, the states of $S$ represents a predicted positive or negative future value of the modelled asset price (smoothed by the moving average). As $S$ represents a future value, evidence for $S$ was only available during estimation of the conditional and marginal distributions contained within the NBC, not during the generation of signals.

\section{Bayesian optimisation settings}

For the two structures GBN-1 and GBN-2, we had the following free parameters $\Lambda$. In both cases all $\tau$ were confined to $[0.6,0.9]$ and all $\psi$ to $[10,40]$.

- For GBN-1: $\Lambda=\left\{\tau_{1}, \tau_{2}, \psi_{\text {Buy }}, \psi_{\text {Sell }}\right\}$.

- For GBN-2: $\Lambda=\left\{\tau_{1}, \tau_{2}, \tau_{3}, \tau_{4}, \psi_{\text {Trend }}, \psi_{\text {Long }}, \psi_{\text {Short }}\right\}$.

We used the upper confidence bound acquisition function (as described in Section 3.4.2 with $\eta=5$, which allowed for abundant exploration, as our objective function was not extremely expensive to evaluate. We used the rational quadratic kernel with $c=1$. For GBN-1 we ran the Bayesian optimisation for 1600 iterations, and for GBN-2 we ran 12800 iterations.

\section{Data sets}

We used four indices in this study, DJIA and NASDAQ which are both based on companies in the United States, FTSE100 which is based on companies in the United Kingdom and DAX which is based on companies located in Germany. We ran our experiments on daily adjusted closing prices for these indices ranging from 2001-01-01 to 2012-12-28 (data downloaded from Yahoo! Finance $\left.{ }^{\mathrm{TM}}\right)$. This gave a total of 12 years of price data for each index, where each year was allocated to a block, thus $n=12$. For the crossvalidation step we used $k=5$ giving $t=7$ simulations from which to calculate $\left[\bar{\rho}_{\mathcal{J}_{1}}, \ldots, \bar{\rho}_{\mathcal{J}_{m}}\right]$.

\footnotetext{
${ }^{2}$ Using the mean and one and two standard deviations above and below the mean as cutoff points.
} 
Table 3.4: Metric values for GBNs and BaH used for index trading.

\begin{tabular}{|ll||lllll|}
\hline & & Sharpe & Return & MDD & LVFI & TIMR \\
\hline DJIA & GBN-1 & 0.289 & 0.019 & $\mathbf{0 . 0 8 5}$ & $\mathbf{0 . 0 5 8}$ & $\mathbf{0 . 6 2 8}$ \\
& GBN-2 & $\mathbf{0 . 3 3 0}$ & $\mathbf{0 . 0 3 2}$ & 0.116 & 0.062 & 0.910 \\
& BaH & 0.157 & 0.028 & 0.167 & 0.119 & 1.000 \\
\hline NASDAQ & GBN-1 & $\mathbf{0 . 3 0 8}$ & 0.033 & $\mathbf{0 . 1 0 1}$ & $\mathbf{0 . 0 6 2}$ & $\mathbf{0 . 5 5 4}$ \\
& GBN-2 & 0.081 & 0.012 & 0.164 & 0.099 & 0.940 \\
& BaH & 0.254 & $\mathbf{0 . 0 6 7}$ & 0.207 & 0.146 & 1.000 \\
\hline FTSE100 & GBN-1 & -0.057 & -0.006 & $\mathbf{0 . 0 9 8}$ & $\mathbf{0 . 0 7 4}$ & $\mathbf{0 . 6 4 9}$ \\
& GBN-2 & -0.640 & -0.074 & 0.167 & 0.121 & 0.962 \\
& BaH & $\mathbf{0 . 1 2 7}$ & $\mathbf{0 . 0 2 2}$ & 0.188 & 0.142 & 1.000 \\
\hline DAX & GBN-1 & $\mathbf{0 . 7 7 8}$ & $\mathbf{0 . 0 8 1}$ & $\mathbf{0 . 1 0 7}$ & $\mathbf{0 . 0 5 6}$ & $\mathbf{0 . 6 1 0}$ \\
& GBN-2 & 0.589 & 0.062 & 0.171 & 0.059 & 0.926 \\
& BaH & 0.278 & 0.069 & 0.213 & 0.154 & 1.000 \\
\hline
\end{tabular}

\section{Scoring functions}

The signals generated were backtested in order to calculate relevant metrics. During optimisation the objective function used the cross-validation estimate of the Sharpe ratio. For evaluating the performance of the optimisation we used the Sharpe ratio, return and the drawdown risks described in Section 3.1 .1 to create a score vector $\left[\bar{\rho}_{\mathcal{J}_{1}}, \ldots, \bar{\rho}_{\mathcal{J}_{m}}\right]$. The same metrics were calculated for the $\mathrm{BaH}$ strategy.

\subsubsection{Results and discussion}

The score vectors from the evaluation of the proposed learning algorithm versus the score vector for the $\mathrm{BaH}$ strategy over the seven simulations are shown in Table 3.4. The annual Sharpe presented in the table is the mean return divided by the standard deviation of returns over the seven simulation, and since each block was allocated one year of data it becomes the annual Sharpe ratio.

We will first turn our attention to GBN-1. We use the Sharpe ratio as our measure of reward, prioritised above the raw return for reasons discussed in Section 3.1.1. Therefore, we must first ensure that the Sharpe ratio of our algorithmic trading system produces similar or better Sharpe ratios than the $\mathrm{BaH}$ strategy. As can be seen, this was the case for DJIA, NASDAQ and DAX, but not for FTSE100. Secondly, we must take into consideration the TIMR. For GBN-1, we were invested only slightly above half of the time compared to $\mathrm{BaH}$, reducing risk to equity considerably. Meanwhile, the rest of the time the equity could have gained in value from interest rates (or other risk free assets), this potential gain was not considered in these results. Risk to equity from MDD was half its counterpart from the $\mathrm{BaH}$ strategy for all indices. As we have previously discussed, the LVFI is a major threat to equity, 
and one where BaH severely underperforms. For DAX the LVFI was only a third of the BaH LVFI, and for the other three indices it was half.

All in all, the results clearly indicate that GBN-1 was competitive with the $\mathrm{BaH}$ strategy for three of the indices, as Sharpe ratios were improved upon and risk to equity was decreased significantly. Furthermore, these results were achieved while at the same time only having equity invested half of the investment period. It is also clear that we cannot expect the same setup to be useful for all indices, as the reward was not improved upon for FTSE100. Some of the parameters that were fixed in our GBNs may have to be tuned in order to accommodate the dynamics of FTSE100, such as the technical analysis indicators used, or the fixed parameters of the ones currently used.

Moving on to GBN-2, we can see that allowing the GBN to open short positions changes the results dramatically. For DJIA, we improved upon the Sharpe ratio, at the cost of the drawdown risks. Both MDD and LVFI were increased marginally, yet still lower than BaH. The TIMR was also increased to such a degree that we were invested almost the entire investment period. There is potential gain in reward from using GBN-2 for DJIA, however the increased risk must be considered.

For NASDAQ, FTSE100 and DAX there was no improvement over GBN1. Instead, Sharpe ratios are decreased, as well as an increase in drawdown risks. There could be several reasons for this that are worth investigating, however two important factors to consider are that a bad short position is doubly bad on equity as we will lose out of the profit from a long position during the same time, and the long term trend in stock markets is generally positive, thus taking positions against this long term trend is generally illadvised.

\subsection{Conclusions and summary}

While the BaH strategy may be superior to GBNs in terms of raw return (as can be seen from Tables 3.1 and 3.4, the risks that this strategy pose in the interim are non-negligible. We would like to think of the return that our strategies generate as somehow accessible, i.e. being available at any time, and not a consequence of long term investment. Figuratively speaking, we would like our equity curves to be monotonic, without taking deep drawdowns (in the form of MDD or LVFI). The experiments in this chapter have shown how we can use GBNs to define a strategy for buying and selling assets such that we substantially reduce risks towards invested capital. 
We have introduced two learning algorithms that can be used to learn GBNs with the aim of encoding a strategy that optimises some score. The template based approach allows us to use expert knowledge to construct the BNs that should be contained, and then to find the combination of BNs that maximise the score. Understandably this type of expert knowledge is not always available, thus we have offered a second algorithm that can (to some degree) reduce the reliance on expert knowledge, while at the same time deal with more complex GBN structures.

In Chapter 4 we shall move away from learning GBNs which contain BNs with different variables, and instead focus on GBNs that contain BNs where the variables stay the same but the edges change (i.e. the independencies are different among the BNs). We shall no longer have a GBN structure given a priori, but will instead attempt to discover the GBN structure from data, and the goal will no longer be to increase a score function, but rather to create a representative model of the system under observation. 



\section{Chapter 4}

\section{Detecting regimes using gated Bayesian networks}

In Chapter 3 we used GBNs to model a specific process, or put another way we tried to imitate or mimic the decision making process of a financial asset trader. In the cases that we presented the structure of the process was given a priori, i.e. the number of BNs and gates and their connections was fixed before we started estimating the free parameters of the models. This allowed us to learn GBNs targeted for a specific outcome (for instance, improving the expected annual Sharpe ratio above the $\mathrm{BaH}$ strategy). In this chapter one of the central themes will be the identification of the process structure itself. The GBNs that we shall learn will no longer be used for a specific outcome, but will rather be generative in their nature. For this purpose we shall no longer consider different variables among the contained BNs, but rather different conditional independencies. For instance, the GBN in Figure 2.6c contains two BNs with the same random variables, but where the edges instead differ.

In Section 4.1 we shall begin by introducing some terminology that we shall use throughout the chapter, and also more clearly state the aim of the learning algorithm that we shall introduce in Section 4.2. In Section 4.3 we shall comment on work that is related to the approach we are taking. Then in Section 4.4 we shall account for the first of two sets of experiments using synthetic data. In Section 4.5 we shall turn our attention to a real-world application of the learning algorithm, specifically using GBNs to model baseball players' career data (this section will also include the second set of experiments on synthetic data). Before finishing this chapter with our conclusions in Section 4.7 we shall look at another set of real-world experiments using financial data in Section 4.6 , 


\subsection{Regime changes and gated Bayesian networks}

When we observe a probabilistic system ${ }^{1}$ over time, it is natural that the observations we make differ from each other. We expect this to be the case due to the randomness of the variables within the system. However, as long as the relationships between the variables, and the distributions of the individual variables are unchanged, the observations we make of the system can be judged as coming from the same joint probability distribution.

Let $X, Y$ and $Z$ be three random variables, and let there exist a joint probability distribution $p(X, Y, Z)$. We can factorise this joint distribution using the chain rule to get $p(X, Y, Z)=p(X \mid Y, Z) p(Y \mid Z) p(Z)$. If we also know that $X$ is independent of $Y$ given $Z(X \Perp Y \mid Z)$, then the factorisation can be reduced to $p(X, Y, Z)=p(X \mid Z) p(Y \mid Z) p(Z)$, where $Y$ has been dropped from the conditioning set in the distribution of $X$, since they are independent given $Z$.

However, over time the relationships among the variables may change. Assume that after some time $X \Perp Y \mid Z$ no longer holds in the system. This implies that the factorisation can not be reduced, and therefore $p(X, Y, Z)$ prior to the change and after the change are not the same. Observations before the change and after the change should no longer be judged to be samples from the same joint probability distribution.

While we will not delve into deeper detail why such changes may occur, the phenomenon of regime changes has been studied extensively in the fields of ecology [60, 61, 62], economy and finance [63, 64, 65, 66, 67], and biology [68, 69]. Given the diversity of fields studying regimes, there is no general consensus of what a regime entails, nor its length or abruptness. However, a non-conflicting definition is to say that a regime is a steady state of a system. We define this steady state as one where all probabilistic relationships among the variables in a system stay the same. A system can exhibit several regimes, between which the probabilistic relationships may change. We will also consider regime changes to be both infrequent and abrupt, as opposed to the related notion of concept drift [17], where changes are smooth. Thus, we do not expect a system to change regimes very often, and we assume that the changes between regimes is immediate.

\footnotetext{
${ }^{1}$ We choose to use the word system rather than set of random variables here since it is more natural to think about some system that we observe over time. However, there is no difference between what we here call a system and a set of random variables.
} 


\subsubsection{Notation}

Let $\mathcal{S}$ be a system of random variables $\mathbf{X}$, and let the system $\mathcal{S}$ have regimes labeled $R_{1}, R_{2}, \ldots, R_{m}$. For each regime $R_{k}$ there exists an independence model $M_{k}$ over the variables $\mathbf{X}$ and an associated joint probability distribution $p_{k}(\mathbf{X})$. Furthermore, assume that $M_{i} \neq M_{j} \forall i \neq j$. Let $\mathcal{D}$ be a data set with complete observations of $\mathbf{X}$, where $d_{i}$ is the $i$ :th data point in $\mathcal{D}$.

By $d_{i} \sim R_{k}$ we mean that $d_{i}$ is a data point containing observations of the variables $\mathbf{X}$ when $\mathcal{S}$ is in regime $R_{k}$, thus $d_{i}$ is a sample from $p_{k}(\mathbf{X})$. We will for brevity say that $d_{i}$ comes from regime $R_{k}$. Then $\mathcal{D}=\left\{d_{1} \sim\right.$ $\left.R_{1}, d_{2} \sim R_{1}, d_{3} \sim R_{1}, d_{4} \sim R_{2}, d_{5} \sim R_{2}\right\}$ means that the first three data points came from regime $R_{1}$ while the last two data points came from regime $R_{2}$. When there is no ambiguity we will shorten $\mathcal{D}=\left\{d_{1} \sim R_{1}, d_{2} \sim\right.$ $\left.R_{1}, d_{3} \sim R_{1}, d_{4} \sim R_{2}, d_{5} \sim R_{2}\right\}$ to $\mathcal{D}=\left\{R_{1}, R_{1}, R_{1}, R_{2}, R_{2}\right\}$.

Given $\mathcal{D}=\left\{R_{1}, R_{1}, R_{1}, R_{2}, R_{2}\right\}$ it is possible to directly identify which regimes that can transition into each other, i.e. in this case $\mathcal{S}$ can transition from $R_{1}$ to $R_{2}$. We call this the regime transition structure of $\mathcal{S}$, which can be drawn as a graph or denoted with $R_{1} \rightarrow R_{2}$. Had the observations been different, such that $\mathcal{D}=\left\{R_{1}, R_{1}, R_{1}, R_{2}, R_{2}, R_{1}, R_{1}\right\}$, then this would have identified a different structure where $R_{1}$ can transition to $R_{2}$, and $R_{2}$ can transition to $R_{1}\left(R_{1} \leftrightarrows R_{2}\right)$. It is necessary to assume a Markovian property of the regime transitions, such that knowing that $\mathcal{S}$ is in regime $R_{i}$ is the only necessary information in order to identify which regimes it can transition into. We will defer the reasoning and consequences of this Markovian assumption to Section 4.2.2.

We say that $\mathcal{D}$ is a data set for $\mathcal{S}$ if it identifies the true regime transition structure of $\mathcal{S}$, i.e. $\mathcal{D}$ is a valid sample of $\mathcal{S}$. For instance, if the true regime structure of $\mathcal{S}$ is $R_{1} \rightarrow R_{2}$ then $\mathcal{D}=\left\{R_{1}, R_{2}\right\}$ is a data set for $\mathcal{S}$, while $\mathcal{D}=\left\{R_{1}\right\}, \mathcal{D}=\left\{R_{2}\right\}, \mathcal{D}=\left\{R_{2}, R_{1}\right\}$, and $\mathcal{D}=\left\{R_{1}, R_{2}, R_{1}\right\}$ are not. It is implied that data sets are made available to us completely and immediately. However, we will also make use of data points that are given to us one by one as $\mathcal{S}$ is observed over time. We call this a stream of data. The size of a stream $\mathcal{O}$ depends on how many observations we have made thus far: at time $t=1$ the stream only contains a single data point, e.g. $\mathcal{O}=\left\{d_{1} \sim R_{1}\right\}$, and at time $t=j$ it contains $j$ data points, e.g. $\mathcal{O}=\left\{d_{1} \sim R_{1}, \ldots ., d_{j} \sim R_{k}\right\}$.

\subsubsection{Aim}

Given a data set $\mathcal{D}=\left\{d_{1}, \ldots, d_{n}\right\}$ for $\mathcal{S}$, where it is not known from which regime the individual data points came from, and it is not known how many 
regimes $\mathcal{S}$ exhibits, the primary aim is to learn a model of $\mathcal{S}$ from $\mathcal{D}$. In order to do so we must:

- Identify where in $\mathcal{D}$ there are regime changes.

- Identify the regimes $R_{1}, \ldots, R_{m}$ of $\mathcal{S}$, and their corresponding independence models $M_{1}, \ldots, M_{m}$ and joint distributions $p_{1}(\mathbf{X}), \ldots, p_{m}(\mathbf{X})$.

- Identify the regime transition structure of $\mathcal{S}$.

Once such a model has been defined, the secondary aim is to take a stream of data $\mathcal{O}$ and correctly identify which regime $\mathcal{S}$ currently is in, given every new data point in $\mathcal{O}$. In order to do so we must extend our model to allow it to detect regime changes in $\mathcal{O}$. This will result in a model that can be used to reason about the current regime of $\mathcal{S}$, and also to identify which $M_{k}$ and $p_{k}(\mathbf{X})$ should be used for inference purposes.

We will meet the primary and secondary aim by learning a GBN. By using a GBN to represent a system that has several regimes, we can model each regime using a $\mathrm{BN}$, and then connect them with gates according to the regime transition structure. We can also define the trigger logic in such a way that the model is capable of, given a stream of observations, accurately keeping the BN active that represents the current regime. Therefore, a GBN is a model that can incorporate both of the present aims, and to this end we will suggest an algorithm in Section 4.2 to learn such GBNs from data.

\subsection{Learning algorithm}

In this section we will describe the algorithm that we propose in order to learn a GBN that fulfils the primary and secondary aim of the problem definition in Section 4.1.2. Pseudocode for the entire algorithm is offered in Appendix A. There are three major steps involved, which we will briefly outline here, and then discuss in the rest of this section:

1. The GBN that we are learning consists of a BN for each regime. We shall partition a data set $\mathcal{D}$ into subsets, treating each subset as data points from a regime, and learn the structure and parameters of a $\mathrm{BN}$ for each subset. The first step of the algorithm is to find the partitioning that represents the regime changes that occurred during the collection of $\mathcal{D}$. We will to this end assume that data points within each subset are independent and identically distributed, and that we can calculate the likelihood of the entire model by the product of the likelihoods of the contained BNs. 
2. Since we only detect regime changes in the first step, the resulting regime transition structure is a chain of regimes, i.e. no regimes reoccur. However we are also interested in identifying reoccurring regimes, and we will therefore hypothesise mergers of the identified subsets, to find the mergers that lead to the most likely regime transition structure.

3. The final step of the learning algorithm is to introduce gates between the identified regimes, and to optimise the required parameters of these gates. The goal is to be able to use the GBN on a stream of data points, and for each new data point decide which regime the system currently is in.

The rest of this section describes these three steps in detail.

\subsubsection{Identifying regime changes in the data set}

In order to identify where regime changes have occurred in a given data set, we use Metropolis-Hastings MCMC (MH). A typical Bayesian feature selection method is employed, where we have $k$ splits $\delta_{1}, \ldots, \delta_{k}$, each defined by their position in the data set $\beta_{1}, \ldots, \beta_{k}$, and an indicator variable $I_{1}, \ldots, I_{k}$, that is either 0 or 1 . By defining $\delta_{i}=I_{i} \beta_{i}$ the splits can move along the data set by changing the corresponding $\beta$, and be turned on and off by the corresponding $I$. For a certain configuration of $\beta$ s and Is we specify a model by learning a $\mathrm{BN}$ for each subset of the data defined by the $\delta$ s that are nonzero. We want to estimate the values of the $\delta$ s, given the available data, and therefore we are interested in the posterior distribution over the $\beta$ s and Is given a data set $\mathcal{D}$ with $n$ observations. That is, we need samples from the posterior distribution in Equation 4.1

$$
\begin{aligned}
& p\left(\beta_{1}, \ldots, \beta_{k}, I_{1}, \ldots, I_{k} \mid \mathcal{D}\right) \propto \\
& p\left(\mathcal{D} \mid \beta_{1}, \ldots, \beta_{k}, I_{1}, \ldots, I_{k}\right) U\left(\beta_{1} ; 0, \beta_{2}\right) U\left(\beta_{2} ; \beta_{1}, \beta_{3}\right) \times \\
& U\left(\beta_{k} ; \beta_{k-1}, n+1\right) p\left(I_{1}\right) \ldots p\left(I_{k}\right)
\end{aligned}
$$

As is evident from Equation 4.1, we will a priori assume that Is are independent, and that $\beta$ s are distributed according to a discrete uniform distribution, where $U(a ; b, c)$ represents a discrete uniform distribution over $a$ with the range $(b, c)$. A Bernoulli distribution is used as prior for each $I$, which is parameterised with $p=0.5$ to represent how likely each split is a priori. 


\section{Likelihood derivation}

In order to sample from the posterior in Equation 4.1, we must be able to calculate the marginal likelihood of the data, $p\left(\mathcal{D} \mid \beta_{1}, \ldots, \beta_{k}, I_{1}, \ldots, I_{k}\right)$. To do this we use the following restructuring:

- Let $\left\{\gamma_{1}, \ldots, \gamma_{k^{\prime}}\right\}$ represent the subset of $\left\{\delta_{1}, \ldots, \delta_{k}\right\}$ for which the corresponding $I_{1}, \ldots, I_{k}$ are equal to one, i.e. $\left\{\gamma_{1}, \ldots, \gamma_{k^{\prime}}\right\}$ represents the nonzero $\delta \mathrm{s}$. We will first assume that $k^{\prime}>1$, the two cases of $k^{\prime}=0$ and $k^{\prime}=1$ will be addressed subsequently.

- Let $\mathcal{D}_{\gamma_{i}}^{\gamma_{j}-1}$ represent observations $d_{\gamma_{i}}, \ldots, d_{\gamma_{j}-1}$ where $i<j$.

- We can then write the marginal likelihood expression as:

$$
\begin{aligned}
& p\left(\mathcal{D} \mid \beta_{1}, \ldots, \beta_{k}, I_{1}, \ldots, I_{k}\right)= \\
& p\left(\left\{\mathcal{D}_{1}^{\gamma_{1}-1}, \mathcal{D}_{\gamma_{1}}^{\gamma_{2}-1}, \ldots, \mathcal{D}_{\gamma_{k^{\prime}}}^{n}\right\} \mid \gamma_{1}, \ldots, \gamma_{k^{\prime}}\right)
\end{aligned}
$$

- Since we have assumed that a different model holds for each subset, and that we can calculate the marginal likelihood of the entire model by the product of the contained models, we can further rewrite the expression from Equation 4.3 .

$$
\begin{aligned}
& p\left(\left\{\mathcal{D}_{1}^{\gamma_{1}-1}, \mathcal{D}_{\gamma_{1}}^{\gamma_{2}-1}, \ldots, \mathcal{D}_{\gamma_{k^{\prime}}}^{n}\right\} \mid \gamma_{1}, \ldots, \gamma_{k^{\prime}}\right)= \\
& p\left(\mathcal{D}_{1}^{\gamma_{1}-1} \mid \gamma_{1}\right) p\left(\mathcal{D}_{\gamma_{1}}^{\gamma_{2}-1} \mid \gamma_{1}, \gamma_{2}\right) \ldots p\left(\mathcal{D}_{\gamma_{k^{\prime}}}^{n} \mid \gamma_{k^{\prime}}\right)
\end{aligned}
$$

- We shall use a BN for each subset, thus the full Bayesian approach of evaluating each factor on the right hand side in Equation 4.3 would be to sum over all BNs, e.g.:

$$
p\left(\mathcal{D}_{1}^{\gamma_{1}-1} \mid \gamma_{1}\right)=\sum_{B N_{i} \in \mathbf{B N}} p\left(\mathcal{D}_{1}^{\gamma_{1}-1} \mid B N_{i}, \gamma_{1}\right) p\left(B N_{i} \mid \gamma_{1}\right)
$$

However, this is unfortunately impractical and therefore we shall approximate each factor by using the maximum a posteriori (MAP) BN, obtained by using a learning algorithm $\mathcal{L}$. Let $\mathcal{L}\left(\gamma_{i}, \gamma_{j}-1\right)$ represent the BN learnt using algorithm $\mathcal{L}$ and data set $\mathcal{D}_{\gamma_{i}}^{\gamma_{j}-1}$. Then the MAP approximation ${ }^{2}$ to the full Bayesian approach is given by:

\footnotetext{
${ }^{2}$ In the current setting, using the MAP approximation is rather convenient, since it gives us a $\mathrm{BN}$ for each regime, and it is these BNs that combine into the full GBN.
} 


$$
\begin{aligned}
& p\left(\mathcal{D} \mid \beta_{1}, \ldots, \beta_{k}, I_{1}, \ldots, I_{k}\right)= \\
& p\left(\mathcal{D}_{1}^{\gamma_{1}-1} \mid \mathcal{L}\left(1, \gamma_{1}-1\right)\right) p\left(\mathcal{D}_{\gamma_{1}}^{\gamma_{2}-1} \mid \mathcal{L}\left(\gamma_{1}, \gamma_{2}-1\right)\right) \ldots \times \\
& p\left(\mathcal{D}_{\gamma_{k^{\prime}}}^{n} \mid \mathcal{L}\left(\gamma_{k^{\prime}}, n\right)\right)
\end{aligned}
$$

In the special case when $k^{\prime}=1$, only the first and last factor of Equation 4.5 applies, and when $k^{\prime}=0$ we have $p\left(\mathcal{D} \mid \beta_{1}, \ldots, \beta_{k}, I_{1}, \ldots, I_{k}\right)=$ $p(\mathcal{D} \mid \mathcal{L}(1, n))$.

From Equation 4.5 we can deduce that to calculate the marginal likelihood in Equation 4.1, we must calculate the marginal likelihood of the subset of data used to learn the corresponding BN. As discussed in Section 2.1.3, we can for discrete BNs calculate the marginal likelihood of a BN structure via Equation 2.7.

\section{Proposal distributions}

The MH simulation is initialised by setting all $\beta$ s so that the $\delta$ s are evenly spaced out across the data set, and all Is are set to 1 . Proposing new values for the indicator variables $I$ is done via a Bernoulli distribution with $p=0.5$, so that it is equally likely that the $I$ will change or that it will stay the same. The $\beta$ values require a bit more work, as there are constraints on how we want them to be proposed.

First of all, the $\beta$ s have to be proposed within the bounds of the data set, i.e. between 1 and $n$. Secondly, we want $\beta_{i}<\beta_{j}$ for all $i<j$, so that two $\beta$ s never collide or jump over each other. Finally, we want the $\beta$ s to have a positive probability of taking on any value between their respective upper and lower bounds. Since the $\beta$ s are positions in a data set they are discrete values, thus we can create a proposal distribution for each $\beta$ according to Equations 4.6 through 4.10 . In the equations, $\beta_{j}$ is the current value while $\beta_{j}^{*}$ is the proposed value. In Equation 4.6 and 4.7 we define the upper and lower bound for $\beta_{j}^{*}$, in such a way that $\beta_{j}^{*}$ always is proposed within the data set, and so that $\beta_{j}^{*}$ never can be proposed as the same value as any of the other $\beta$ s. In Equation 4.8 and 4.9 , each allowed value $i$ of $\beta_{j}^{*}$ is given a probability that is proportional to its distance from the current value $\beta_{j}$. $\mathrm{Z}$ is a normalisation constant defined in Equation 4.10 . 


$$
\begin{aligned}
& l b\left(\beta_{j}^{*}\right)= \begin{cases}1 & \text { if } j=1 \\
\beta_{j}-\left\lfloor\frac{1}{2}\left(\beta_{j}-\beta_{j-1}\right)\right\rfloor-1 & \text { otherwise }\end{cases} \\
& u b\left(\beta_{j}^{*}\right)= \begin{cases}n & \text { if } j=k \\
\beta_{j}+\left\lfloor\frac{1}{2}\left(\beta_{j+1}-\beta_{j}\right)\right\rfloor-1 & \text { otherwise }\end{cases} \\
& \kappa=\max \left(\beta_{j}-l b\left(\beta_{j}^{*}\right), u b\left(\beta_{j}^{*}\right)-\beta_{j}\right) \\
& p\left(\beta_{j}^{*}=i \mid \beta_{j}\right)= \begin{cases}\frac{1}{Z}\left(1+i-\beta_{j}+\kappa\right) & \text { for } l b\left(\beta_{j}^{*}\right) \leqslant i \leqslant \beta_{j} \\
\frac{1}{Z}\left(1-i+\beta_{j}+\kappa\right) & \text { for } \beta_{j}<i \leqslant u b\left(\beta_{j}^{*}\right)\end{cases} \\
& \mathrm{Z}=\sum_{i=l b\left(\beta_{j}^{*}\right)}^{\beta_{j}}\left(1+i-\beta_{j}+\kappa\right)+\sum_{i=\beta_{j}+1}^{u b\left(\beta_{j}^{*}\right)}\left(1-i+\beta_{j}+\kappa\right)
\end{aligned}
$$

To visualise this, assume that there are two $\beta \mathrm{s}$ and $n=39$. In the top left plot in Figure 4.1 the current values are $\beta_{1}=10$ and $\beta_{2}=30$. As can be seen, $\beta_{1}^{*}$ and $\beta_{2}^{*}$ can be proposed in both directions within their respective bounds. In the top right plot the two $\beta \mathrm{s}$ are positioned at 1 and 39 respectively, and are now constrained to move in only one direction due to the data set bounds. In the bottom left plot the two $\beta$ s are positioned at 15 and 25 , and points that they have in common in their range are removed from both proposals. The bottom right plot shows the case where the $\beta$ s are positioned at 19 and 21 , and there is no probability of the $\beta$ s moving closer to each other.

\section{Acceptance probability}

To make sure that we asymptotically are sampling from the posterior distribution in Equation 4.1, we have to correct for the fact that the proposal distribution over the $\beta \mathrm{s}$, defined by Equations 4.6 through 4.10 , is asymmetric. That is, it is not guaranteed that we have $p\left(\beta_{j}^{*} \mid \beta_{j}\right)=p\left(\beta_{j} \mid \beta_{j}^{*}\right)$ for Equation 4.9. $\mathrm{MH}$ adjusts for this when deciding to keep or reject the proposed parameters [70, 71]. The probability of moving from the current parameters $I$ and $\beta$ to the proposed parameters $I^{*}$ and $\beta^{*}$ can be expressed as $p\left(\beta^{*} \mid \beta\right) p\left(I^{*} \mid I\right)=\frac{1}{2 k} p\left(\beta^{*} \mid \beta\right)$. The acceptance ratio $r$ is then calculated by using Equation 4.11, and the proposed parameters are accepted with probability $\min (r, 1)$.

$$
r=\frac{p\left(\beta^{*}, I^{*} \mid \mathcal{D}\right) / \frac{1}{2 k} p\left(\beta^{*} \mid \beta\right)}{p(\beta, I \mid \mathcal{D}) / \frac{1}{2 k} p\left(\beta \mid \beta^{*}\right)}
$$



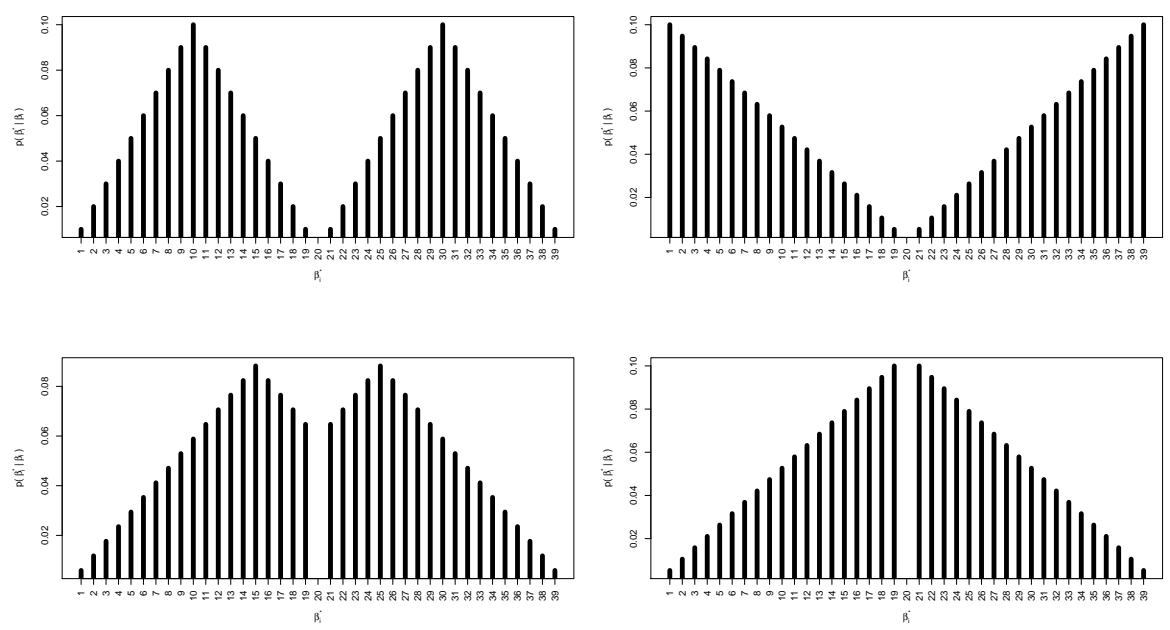

Figure 4.1: Example proposal distributions for $\beta \mathrm{s}$.

\section{Iterations and samples}

While it is possible to monitor the MH iterations to decide when to stop sampling, we run the simulation for a fixed number of iterations and throw away the first half of the samples (treating them as burn-in samples). We use the mean of the marginal of each scalar parameter $\beta$ and $I$ and round to integer values, i.e. if the marginal mean of $I_{j}$ is 0.4 we will round this to 0 and if it is 0.6 we will round it to 1 . The resulting nonzero $\delta$ s then identify where in the data set regime changes have occurred.

\subsubsection{Identifying regimes and structure}

Having identified nonzero $\delta$ s where regime changes have occurred in a data set $\mathcal{D}$, the next step is to identify the individual regimes, as well as the regime transition structure of the underlying system. Naïvely assuming that each change identifies a new regime would lead to a chain of regimes, i.e. if there were two nonzero $\delta$ s identified we would have $R_{1} \rightarrow R_{2} \rightarrow R_{3}$. While it may be entirely possible for a system to exhibit this type of regime structure, it is also possible that $R_{2}$ transitioned back to $R_{1}$, and not into a new regime $R_{3}$. Therefore we must hypothesise each possible recursive merging of nonadjacent subsets, as defined by the nonzero $\delta$ s, and score a new model based on these new subsets. Follows does an explanation and example of this merging. 


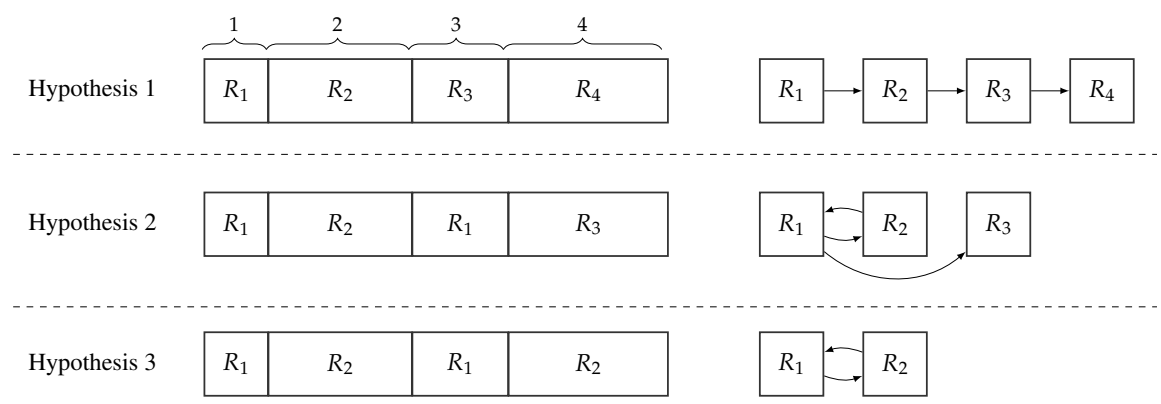

Figure 4.2: Example of merging subsets.

\section{Merging subsets}

In the example in Figure 4.2 we have identified three nonzero $\delta$ s, resulting in four subsets of the data (labeled 1,2,3 and 4). The first hypothesis requires no merging at all; it suggests that each subset identifies a new regime (depicted to the left) and the regime transition structure is therefore a chain of four regimes (depicted to the right). From the first hypothesis we cannot merge subsets 1 and 2, since they are adjacent and we would not have a nonzero $\delta$ here if the two subsets belonged to the same regime. A new hypothesis can however be constructed by merging subsets 1 and 3, resulting in the second hypothesis in the figure. Note that we have now labelled subset 3 with $R_{1}$ and subset 4 with $R_{3}$, as we now only have three regimes rather than four in the previous hypothesis. Should this hypothesis be true, then the regime transition structure must also reflect that from $R_{1}$ it is possible to transition to $R_{3}$, and from $R_{2}$ it is possible to transition to $R_{1}$. Thus when two subsets are merged, we also merge the parent and child sets in the regime transition structure. From the second hypothesis the only merging that can be done is to merge subsets 2 and 4, resulting in the third hypothesis. Note that the example is not complete, as we should go back to the first hypothesis and start the recursive procedure again, but this time by merging subsets 1 and 4 (and similarly so for 2 and 4).

In order to identify the regime structure of a system $\mathcal{S}$, we start by hypothesising the chain of regimes that is given directly from the nonzero $\delta \mathrm{s}$, and then continue to hypothesise each possible recursive merging. For each hypothesis, we merge the subsets of $\mathcal{D}$ accordingly, and learn a new set of BNs using these merged subsets. The learnt BNs are scored according to their marginal likelihood (a simple rephrasing of Equation 4.5). The hypothesis with the highest marginal likelihood, among all the hypotheses, represents the identified regime structure of $\mathcal{S}$. While merging, previously scored 
hypotheses may be created, so the number of hypotheses to score can be substantially pruned. To see this we can continue the merging example from before. This time we start by merging subsets 2 and 4, resulting in subset labelling $R_{1}, R_{2}, R_{3}, R_{2}$, and then we merge subsets 1 and 3 , resulting in $R_{1}, R_{2}, R_{1}, R_{2}$, which is the same as the last hypothesis when we started by merging 1 and 3 .

The number of hypotheses to score is directly connected to the number of nonzero $\delta$ s identified. If there are none, or only one, nonzero $\delta$ then there is no merging possible, resulting in a single hypothesis. If there are two nonzero $\delta$ s there are two hypotheses to score, and with three nonzero $\delta$ s there are five hypotheses to score. If there are $k^{\prime}$ nonzero $\delta \mathrm{s}$, then the number of hypotheses to score equals the number of partitions of $\left\{0, \ldots, k^{\prime}\right\}$ into subsets of nonconsecutive integers (including the partition where each element belongs to its own subset, which we would interpret as a chain of regimes). Following from Theorem 2.1 in [72], the number of hypotheses to score therefore follow the well known Bell numbers (A000110 in OEIS), where the first ten numbers are: $1,1,2,5,15,52,203,877,4140,21147$. By starting with the partition where each element belongs to its own subset, and then recursively merging two subsets (without violating the constraint that the subsets must not contain consecutive numbers), we will visit each allowed partition, thus each possible hypothesis will be scored.

\section{Markovian assumption}

Merging the parent and child sets of the regime transition structure is only valid if we assume that the transitions are first order Markovian. For instance, in the second hypothesis in Figure 4.2 we assume that the system can transition from $R_{1}$ to $R_{3}$, however without the Markovian assumption the data suggests that the system can transition from $R_{1}$ to $R_{3}$ only if it has transitioned to $R_{2}$ previously. If the system only allowed transitions from $R_{1}$ to $R_{3}$ after it had passed through $R_{2}$, then the system would be somehow different depending on if it had gone through $R_{2}$ or not. It would then be arguable that $R_{1}$ prior to $R_{2}$ would not be the same as $R_{1}$ after $R_{2}$, and therefore they would not truly be identical regimes. Hence, it is reasonable to assume this Markovian property of the regime transition structure, allowing us to identify different regime transition structures depending on the most likely hypothesis. 


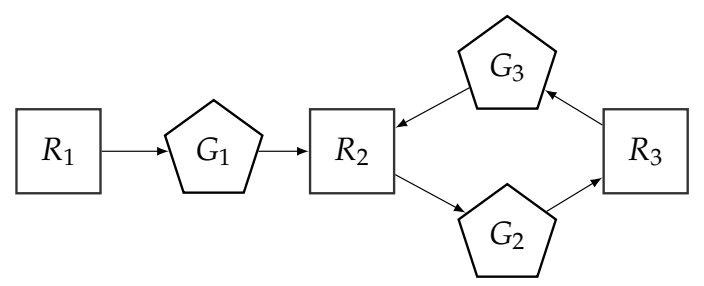

Figure 4.3: Example of a constructed GBN.

\subsubsection{Constructing a gated Bayesian network}

Having identified the regime transition structure, and the BNs that represent each regime, the final step to complete the model of the system is to construct a GBN and configure the gates. The goal is to accept a stream of data points, and for each new data point decide which regime the system currently is in. Following Definitions 5 and 6 from Section 2.2.1, we define a comparison between the parent and the child $\mathrm{BN}$ of each gate. This is done in such a way that if the child is preferred over the parent then the gate triggers, thereby deactivating the parent and activating the child. This comparison is a likelihood ratio between the parent and child using a moving window of the most recent $\tau$ data points. If this ratio is above some threshold $\theta$, then the gate triggers. Let $\mathcal{O}$ be a stream of data points and $\mathcal{O}_{\tau}$ represent the $\tau$ most recent data points, then $p\left(\mathcal{O}_{\tau} \mid R_{1}\right)$ is the likelihood of the most recent $\tau$ data points given regime $R_{1}$. Each gate is then configured to trigger when $p\left(\mathcal{O}_{\tau} \mid R_{\text {child }}\right) / p\left(\mathcal{O}_{\tau} \mid R_{\text {parent }}\right)>\theta$.

For instance, assuming that we have identified the regime structure $R_{1} \rightarrow$ $R_{2} \leftrightarrows R_{3}$, we construct the GBN in Figure 4.3, where the square nodes represents BNs for each regime. For this GBN we define the trigger logic in Equation 4.12, where we use the same $\tau$ and $\theta$ for all gates. The remaining task is then to choose appropriate values for $\tau$ and $\theta$.

$$
\begin{aligned}
T L\left(G_{1}\right) & :=\frac{p\left(\mathcal{O}_{\tau} \mid R_{2}\right)}{p\left(\mathcal{O}_{\tau} \mid R_{1}\right)}>\theta \\
T L\left(G_{2}\right) & :=\frac{p\left(\mathcal{O}_{\tau} \mid R_{3}\right)}{p\left(\mathcal{O}_{\tau} \mid R_{2}\right)}>\theta \\
T L\left(G_{3}\right) & :=\frac{p\left(\mathcal{O}_{\tau} \mid R_{2}\right)}{p\left(\mathcal{O}_{\tau} \mid R_{3}\right)}>\theta
\end{aligned}
$$




\section{Bayesian optimisation and evaluating accuracy}

We shall employ Bayesian optimisation (described in detail in Section 3.4.2) for choosing the parameters $\tau$ and $\theta$. In the current setting, the objective is to find the parameters that maximises the accuracy of the GBN. We say that the GBN is correct if, after processing a new data point in a stream of data, the active $\mathrm{BN}$ corresponds to the true $\mathrm{BN}$ that generated the data point. Thus, if a data point in a stream truly came from $R_{1}$ and the GBN has $R_{1}$ active after processing the data point, then this is considered correct, while any other situation is considered incorrect.

Since we only have access to the training data set during the elicitation process, there is a risk that we may overfit this data. In order to reduce this risk we resample the data points in each identified subset of the original data, and concatenate the resamples to create a new data set of the same size as the original one. Doing so several times we synthetically create new data sets from the original one. As a result of the resampling we can calculate the average accuracy over all resampled data sets for a pair of $\tau$ and $\theta$. Thus, the objective function $f$ that we aim to maximise is therefore the average accuracy of a pair $\tau$ and $\theta$ over all the resamples. Notice that we do not use the true regime labels from the training data, since we assume that these are not known to us, but rather add regime labels based on the regime transition structure identified during subset merging.

Given a GBN with all gates parameterised with some values $\tau$ and $\theta$, and a stream of data $\mathcal{O}$, where each data point is associated with a regime label, the accuracy of the GBN is calculated as follows:

- Activate the $\mathrm{BN}$ that represents $R_{1}$, deactivate all others.

- For the first $\tau-1$ data points from $\mathcal{O}$ do not process any observations, the current regime according to the GBN will be $R_{1}$ for all of these data points. Having less than $\tau$ data points does not allow us to compute the values in Equation 4.12

- Process each data point beyond the first $\tau-1$, the active BN for each data point represents the current regime according to the GBN. Processing of data is done according to the execution algorithm introduced in Section 2.2.3

- When no more data is available, count the number of data points for which the GBN had the correct BN active. 
- The accuracy of the GBN is the number of times the GBN was correct divided by the number of data points in the stream $\mathcal{O}$.

\subsubsection{Summary of learning algorithm}

In Section 4.1.2 we stated that the primary aim is to take a data set $\mathcal{D}$ for a system, where it is not known from which regime the individual data points come from, and learn a model for the system using the data. The secondary aim that we listed is to use the model to correctly identify which regime the system currently is in. We have in the previous sections introduced an algorithm for this purpose, which learns a GBN that models the system and can be used to identify the current regime. Before we evaluate the performance of our proposed algorithm on synthetic experiments, and show how it can be used on real-world data, we shall offer an overview of work related to ours.

\subsection{Related work}

Refining or updating the structure and conditional distributions of a $\mathrm{BN}$ in response to new data has been studied for some time [73, 74, 75, 76]. However, these approaches assume that data is received from a stationary distribution, i.e. a system that does not undergo regime changes.

Nielsen and Nielsen [77] approaches the problem of having a stream of data points which they say is piecewise stationary, i.e. data points within a section of the stream come from the same distribution, but changes may occur into a new stationary section. Their goal is to incrementally learn the structure of a BN, adapting the structure as new data points are made available. They achieve this by monitoring local changes among the variables in the current network, and when a conflict occurs between what is currently learnt and what is observed, they refine the structure of the BN. Focusing only on local changes allows them to reuse all previous data points for parts of the BN that have not changed, resulting in a procedure that is less wasteful and more computationally feasible. Since their goal is to adapt the structure of a single $\mathrm{BN}$, their aim is not to identify reoccurring steady state regimes, but rather to adapt to what is known as concept drift. Our goal is to learn a BN for each regime and to find the regime transition structure of the underlying system. We also intend to use our model to decide which regime is current given a new stream of data, preserving the learnt $\mathrm{BNs}$ for each regime. We do not make the assumption of local change between regimes, but relearn the entire BN, which allows for less assumptions about changes, but as [77] points out can be wasteful of data and computation time. 
Robinson and Hartemink [78] assumes that a complete data set exists, and use MCMC to identify change points to revise a non-stationary DBN (nsDBN). They have several potential moves that they can take within the MCMC iterations, for instance they can shift, split or merge change points, or add/remove an edge in the nsDBN at a change point, etc. By doing so they make the structural learning of the nsDBN an integral part of the MCMC proposals, while our approach plugs in any existing structure learning algorithm. Their aim is to segment the data set and identify which structure was most likely at the different segments, and thereby discovering non-stationary relationships. Our approach is closely related to the approach of Robinson and Hartemink, in the sense that it is the BNs retained after MCMC that represent the model. However, we aim at identifying changes between static regimes, and not to capture the dynamics between timesteps, thus the GBN is not a DBN. Furthermore, Robinson and Hartemink do not approach the problem of trying to identify if regimes reoccur (each segment is considered unique). Therefore the regime transition structure is not captured, and this entails that they do not attempt to use the model on a new data stream in order to predict which of the already learnt structures should be used. Our approach addresses these two latter points.

The nsDBN approach sets itself apart from frameworks such as HMMs and switching state-space models [79] because, as Robinson and Hartemink state, a nsDBN "has no hidden variables, only observed variables". In other words, the nsDBN approach does not assume that there is a hidden random variable representing the regime at each time point. For this reason, modelling the transition probabilities between such random variables, as HMMs and switching state-space models do, does not make sense. Our GBN approach sets itself apart from HMMs and switching state-space models for the same reasons as the nsDBN approach. We shall however conduct parallel experiments using HMMs to contrast our results.

Guo et al. [80] and Xuan and Murphy [81] consider graphs with only undirected edges, i.e. Markov networks rather than BNs. The structure of the Markov network is considered latent by [80], thus their proposed model is a HMM where the states of the latent variable is the entire structure. In order to identify segmentations in time series, [81] score segmentations by computing the marginal posterior over segmentations given all possible decomposable Markov networks. Thus the goal of [81] is to identify the segmentation, rather than the graph. As was pointed out earlier, the GBN that we learn does not contain any hidden variables, and the structure within each regime is explicitly learned. Furthermore, we intend to identify reoccurring regimes and, given new data, predict which regime the system currently is in. 
The concept of regimes is not only confined to the realm of probabilistic graphical models. For instance, Markov regime-switching regression models have been employed in economical studies [63, 64], where one switches the regression coefficients depending on the current regime. For more on adaptation, we refer the interested reader to a recent survey by Gama et al. [17].

\subsection{Synthetic experiments}

In order to demonstrate the feasibility of the proposed learning algorithm described in Section 4.2, we set up a series of simulated experiments. The experiments aimed at showing that, given a data set for a system that exhibits regimes, we can learn a GBN that models the system's regimes and transition structure, and given a new stream of data, the GBN can accurately decide which regime the system currently is in. In this section we shall account for the first set of experiments of two, and defer the second set of experiments to Section 4.5, since they tie into the real-world scenario that we discuss there. Because the HMM is the canonical model for modelling states of the world in the domain of probabilistic graphical models, we also ran these synthetic experiments using standard HMMs and HMMs with different independence models for each of the hidden states. The methodology and results for the HMM experiments can be found in Appendix B.

\subsubsection{Methodology}

We considered data sets with zero to four splits, thus the system generating the data has at minimum one regime and at maximum five. To generate data we created a generic BN with binary variables to represent the first regime in our systems, and then modified this $\mathrm{BN}$ to represent four more regimes. The BNs are presented in Figure 4.4, where $R_{1}$ is the first regime. We randomly generated parameters for the conditional distributions in $R_{1}$. In $R_{2}$ we removed the edge between $C$ and $D$, and since this changed the conditional distribution for $D$ we generated new random parameters for $D$, however we kept the same parameters as in $R_{1}$ for variables where the conditional distributions did not change. Following the same scheme we created regimes $R_{3}$, $R_{4}$ and $R_{5}$.

We created a data set for every possible unique hypothesis for each number of splits, as per the discussion in Section 4.2.2, resulting in 24 different training data sets. Each one of these training data sets is a data set for a system, and we will distinguish between them using the notation $\mathcal{S}_{\text {split,hypothesis }}$, 


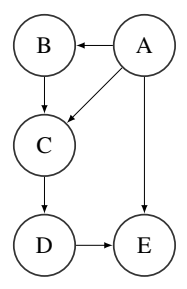

$R_{1}$

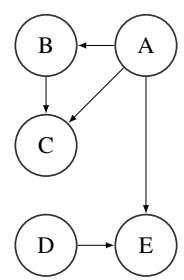

$R_{2}$

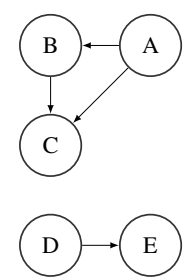

$R_{3}$

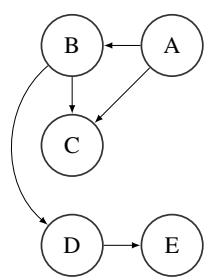

$R_{4}$

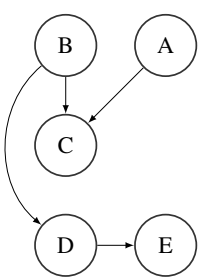

$R_{5}$

Figure 4.4: Five regimes represented by BNs.

e.g. $\mathcal{S}_{3,1}$ is the first hypothesis with three splits and $\mathcal{S}_{4,5}$ is the fifth hypothesis with four splits. Please see Table 4.1 for all systems considered.

The training data sets were created by sampling a random number of samples (uniform between 250 and 600) from each regime, and then concatenating to create a data set. For instance, for the system $\mathcal{S}_{3,2}$ we sampled a random number of samples from $R_{1}$, then a random number of samples from $R_{2}$, then another random number of samples of $R_{1}$ again, and finally a random number of samples from $R_{3}$. We concatenated these samples and treated it as training data for system $\mathcal{S}_{3,2}$. For evaluation purposes we generated another 100 test data sets from each system using the same technique. The test data sets were used as streams of data during accuracy evaluation.

We ran the proposed procedure for each of the 24 training samples, identifying splits, scoring regime transition hypotheses, building GBNs, and optimising gate parameters. To evaluate the performance of the final model we calculated the accuracy over the corresponding 100 streams of test data.

During $\mathrm{MH}$ we used $k=8$ splits, thus we are not assuming any knowledge about how many splits there truly are in the data, only that there are a maximum of eight. We ran 100000 iterations, throwing away the first 50000 samples as burn-in. In order to evaluate Equation 4.5 we needed a BN structure learning algorithm. For this we used the greedy thick thinning algorithm which we introduced in Section 2.1.3, utilising the log marginal likelihood as the target to improve.

We ran 250 iterations of Bayesian optimisation using the radial basis function kernel with $c=2.0$, and the upper confidence bound criterion acquisition function using $\eta=5$. The objective function was the average accuracy over 1000 resamples.

For each of the 24 training samples we report the identified nonzero $\delta \mathrm{s}$, and the distance from these $\delta$ s to the true splits. We also report the average and minimum accuracy over the 100 generated test data sets, as well as the number of test data sets for which the accuracy was below 0.9, 0.8 and 0.7. 


\begin{tabular}{|c|c|c|c|c|c|c|c|c|c|}
\hline 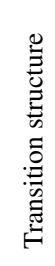 & 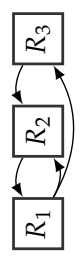 & 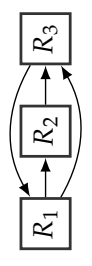 & 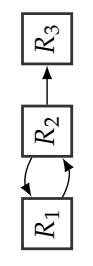 & 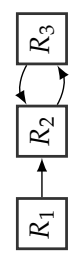 & 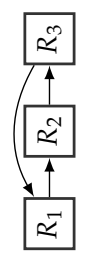 & $\begin{array}{l}\Omega \\
\Omega \\
\Omega \\
\Omega\end{array}$ & & & \\
\hline 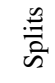 & ナ & ナ & ナ & + & ナ & + & & & \\
\hline 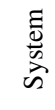 & $\omega^{\circ}$ & $c^{7}$ & $\frac{9}{c^{7}}$ & $\omega^{\frac{m}{f}}$ & $c^{+7}$ & $\underset{c^{+2}}{c^{+2}}$ & & & \\
\hline 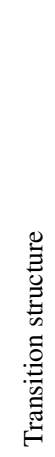 & 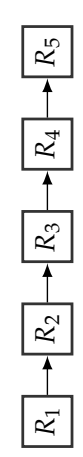 & 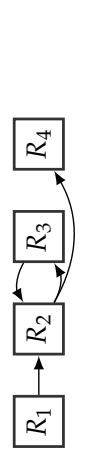 & 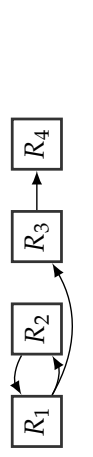 & 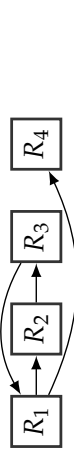 & 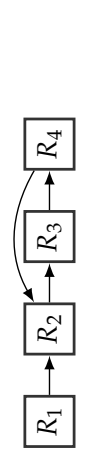 & 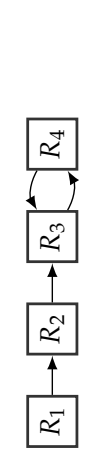 & 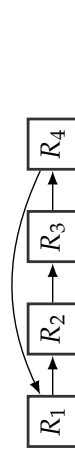 & 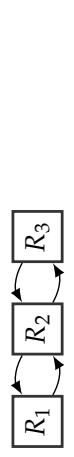 & \begin{tabular}{|l} 
\\
$\Omega$ \\
$\Omega$ \\
$\Omega$ \\
$\Omega$
\end{tabular} \\
\hline 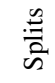 & 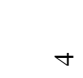 & ナ & + & t & ナ & 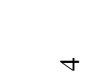 & + & $\checkmark$ & + \\
\hline 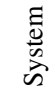 & $0^{7}$ & $\omega^{7}$ & $\omega_{0}^{7}$ & (5) & $c_{0}^{27}$ & $\omega^{0}$ & $\omega^{7}$ & $\omega^{\infty}$ & $\omega^{\circ}$ \\
\hline 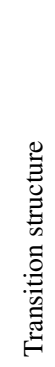 & 固 & 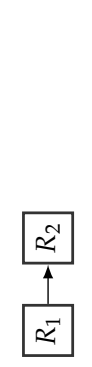 & 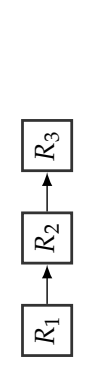 & 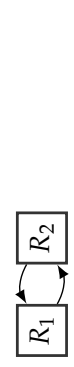 & 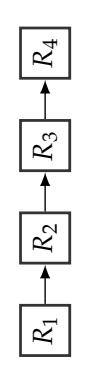 & \begin{tabular}{|l|} 
\\
$\Omega$ \\
$\Omega$ \\
$\Omega$ \\
$\Omega$ \\
\end{tabular} & 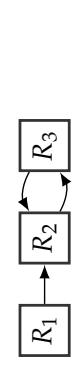 & 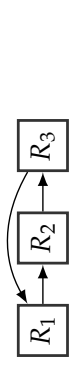 & $\begin{array}{l}\Omega \\
\approx \\
\approx \\
q\end{array}$ \\
\hline 善 & 0 & - & N & $\mathrm{N}$ & $m$ & $m$ & $m$ & $m$ & $m$ \\
\hline 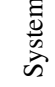 & 0 & $\overrightarrow{0}$ & $\sigma^{\pi}$ & का & co & $\omega^{2}$ & $\omega^{\infty}$ & 泀 & $\omega^{\circ}$ \\
\hline
\end{tabular}


Table 4.2: Actual and learnt splits using synthetic data.

\begin{tabular}{|c|c|c|c|c|c|c|c|c|c|c|c|c|}
\hline System & \multicolumn{4}{|c|}{ True split positions } & \multicolumn{4}{|c|}{ Nonzero $\delta \mathrm{s}$} & \multicolumn{4}{|c|}{ Difference } \\
\hline $\mathcal{S}_{0,1}$ & - & & & & - & & & & - & & & \\
\hline $\mathcal{S}_{1,1}$ & 272 & & & & 272 & & & & 0 & & & \\
\hline $\mathcal{S}_{2,1}$ & 299 & 569 & & & 299 & 572 & & & 0 & 3 & & \\
\hline $\mathcal{S}_{2,2}$ & 436 & 766 & & & 436 & 766 & & & 0 & 0 & & \\
\hline $\mathcal{S}_{3,1}$ & 440 & 796 & 1365 & & 440 & 805 & 1367 & & 0 & 9 & 2 & \\
\hline $\mathcal{S}_{3,2}$ & 281 & 766 & 1351 & & 286 & 767 & 1353 & & 5 & 1 & 2 & \\
\hline $\mathcal{S}_{3,3}$ & 324 & 849 & 1270 & & 321 & 846 & 1263 & & 3 & 3 & 7 & \\
\hline $\mathcal{S}_{3,4}$ & 284 & 535 & 1013 & & 285 & 533 & 1014 & & 1 & 2 & 1 & \\
\hline $\mathcal{S}_{3,5}$ & 579 & 1090 & 1567 & & 578 & 1091 & 1564 & & 1 & 1 & 3 & \\
\hline $\mathcal{S}_{4,1}$ & 385 & 723 & 1166 & 1478 & 386 & 754 & 1177 & 1481 & 1 & 31 & 11 & 3 \\
\hline $\mathcal{S}_{4,2}$ & 582 & 1102 & 1701 & 2153 & 582 & 1115 & 1663 & 2155 & 0 & 13 & 38 & 2 \\
\hline $\mathcal{S}_{4,3}$ & 506 & 827 & 1292 & 1751 & 502 & 830 & 1293 & 1761 & 4 & 3 & 1 & 10 \\
\hline $\mathcal{S}_{4,4}$ & 388 & 711 & 1136 & 1481 & 389 & 718 & 1138 & 1482 & 1 & 7 & 2 & 1 \\
\hline $\mathcal{S}_{4,5}$ & 556 & 1124 & 1534 & 1974 & 554 & 1124 & 1523 & 1977 & 2 & 0 & 11 & 3 \\
\hline $\mathcal{S}_{4,6}$ & 339 & 611 & 1104 & 1533 & 339 & 608 & 1100 & 1539 & 0 & 3 & 4 & 6 \\
\hline $\mathcal{S}_{4,7}$ & 314 & 908 & 1377 & 1947 & 315 & 916 & 1380 & 1949 & 1 & 8 & 3 & 2 \\
\hline $\mathcal{S}_{4,8}$ & 523 & 847 & 1187 & 1714 & 524 & 845 & 1183 & 1715 & 1 & 2 & 4 & 1 \\
\hline $\mathcal{S}_{4,9}$ & 286 & 714 & 1070 & 1623 & 285 & 712 & 1075 & 1624 & 1 & 2 & 5 & 1 \\
\hline $\mathcal{S}_{4,10}$ & 271 & 580 & 1172 & 1583 & 272 & 587 & 1174 & 1570 & 1 & 7 & 2 & 13 \\
\hline $\mathcal{S}_{4,11}$ & 275 & 546 & 963 & 1234 & 280 & 546 & 963 & 1233 & 5 & 0 & 0 & 1 \\
\hline $\mathcal{S}_{4,12}$ & 333 & 746 & 1053 & 1524 & 335 & 741 & 1052 & 1527 & 2 & 5 & 1 & 3 \\
\hline $\mathcal{S}_{4,13}$ & 313 & 719 & 1317 & 1641 & 314 & 724 & 1292 & 1652 & 1 & 5 & 25 & 11 \\
\hline $\mathcal{S}_{4,14}$ & 584 & 916 & 1494 & 1772 & 579 & 913 & 1490 & 1778 & 5 & 3 & 4 & 6 \\
\hline $\mathcal{S}_{4,15}$ & 361 & 893 & 1171 & 1586 & 362 & 895 & 1171 & 1588 & 1 & 2 & 0 & 2 \\
\hline
\end{tabular}

\subsubsection{Results and discussion}

The first step of our experiments was to learn the correct regime transition structure from the training data, and our proposed procedure was in fact able to do so for all 24 systems. From the nonzero $\delta$ s identified during $\mathrm{MH}$, the correct regime transition structure was found following the merging scheme presented in Section 4.2.2. In Table 4.2 we present the true split positions in the training data set, the nonzero $\delta$ s found, as well as the distance between them. As can be seen in the table, for some systems we found the exact split positions, while for others we were slightly off. Note that no splits existed, nor were found, for $\mathcal{S}_{0,1}$.

Interestingly, the learnt structure of the contained BNs did not match exactly the true BN structures. This is not necessarily surprising, as we used rather small samples for the regimes, thus there was not enough data to learn the correct structures for the BNs. However, our procedure was still able to find the correct splits, thus successfully identifying the correct regime transition structure is not contingent on finding the correct BN structures. This may entail that it would suffice using algorithms that learn BN structures that are constrained, such as Chow-Liu trees [82]. 
Table 4.3: Accuracy of learnt GBNs on the test data sets.

\begin{tabular}{|c|l|l|l|l|l|l|l|}
\hline System & $\tau$ & $\theta$ & Avg. acc & Min. acc & $\#<0.9$ & $\#<0.8$ & $\#<0.7$ \\
\hline $\mathcal{S}_{0,1}$ & - & - & - & - & - & - & - \\
\hline $\mathcal{S}_{1,1}$ & 10 & 1.25 & 0.99 & 0.98 & 0 & 0 & 0 \\
\hline $\mathcal{S}_{2,1}$ & 17 & 1.24 & 0.97 & 0.66 & 5 & 3 & 1 \\
\hline $\mathcal{S}_{2,2}$ & 10 & 1.00 & 0.99 & 0.99 & 0 & 0 & 0 \\
\hline $\mathcal{S}_{3,1}$ & 22 & 1.12 & 0.95 & 0.64 & 6 & 3 & 1 \\
\hline $\mathcal{S}_{3,2}$ & 34 & 1.80 & 0.91 & 0.65 & 12 & 10 & 4 \\
\hline $\mathcal{S}_{3,3}$ & 12 & 1.20 & 0.97 & 0.94 & 0 & 0 & 0 \\
\hline $\mathcal{S}_{3,4}$ & 13 & 1.25 & 0.94 & 0.65 & 16 & 9 & 3 \\
\hline $\mathcal{S}_{3,5}$ & 10 & 1.00 & 0.99 & 0.99 & 0 & 0 & 0 \\
\hline $\mathcal{S}_{4,1}$ & 16 & 1.21 & 0.95 & 0.71 & 8 & 4 & 0 \\
\hline $\mathcal{S}_{4,2}$ & 14 & 1.30 & 0.96 & 0.58 & 4 & 1 & 1 \\
\hline $\mathcal{S}_{4,3}$ & 50 & 1.24 & 0.85 & 0.47 & 51 & 18 & 14 \\
\hline $\mathcal{S}_{4,4}$ & 32 & 1.29 & 0.89 & 0.32 & 10 & 9 & 9 \\
\hline $\mathcal{S}_{4,5}$ & 18 & 1.22 & 0.96 & 0.73 & 9 & 2 & 0 \\
\hline $\mathcal{S}_{4,6}$ & 20 & 1.12 & 0.96 & 0.89 & 2 & 0 & 0 \\
\hline $\mathcal{S}_{4,7}$ & 19 & 1.21 & 0.96 & 0.93 & 0 & 0 & 0 \\
\hline $\mathcal{S}_{4,8}$ & 11 & 1.19 & 0.98 & 0.92 & 0 & 0 & 0 \\
\hline $\mathcal{S}_{4,9}$ & 45 & 1.74 & 0.90 & 0.70 & 17 & 6 & 1 \\
\hline $\mathcal{S}_{4,10}$ & 50 & 1.33 & 0.83 & 0.65 & 52 & 46 & 9 \\
\hline $\mathcal{S}_{4,11}$ & 27 & 1.05 & 0.95 & 0.72 & 13 & 6 & 0 \\
\hline $\mathcal{S}_{4,12}$ & 19 & 1.27 & 0.95 & 0.47 & 4 & 2 & 2 \\
\hline $\mathcal{S}_{4,13}$ & 12 & 1.21 & 0.97 & 0.92 & 0 & 0 & 0 \\
\hline $\mathcal{S}_{4,14}$ & 17 & 1.29 & 0.96 & 0.76 & 8 & 2 & 0 \\
\hline $\mathcal{S}_{4,15}$ & 10 & 1.00 & 0.99 & 0.98 & 0 & 0 & 0 \\
\hline
\end{tabular}

The second step was to ensure that the learnt GBNs could accurately activate the current regime given a stream of data. In Table 4.3 we present the $\theta$ and $\tau$ values from the trigger logic of the learnt GBNs (see Section 4.2.3), the average and minimum accuracy over the 100 generated test data sets, as well as the number of test sets where the accuracy was less than 0.9, 0.8 and 0.7. We note that for $\mathcal{S}_{0,1}$ no GBN was learnt, since we correctly did not identify any nonzero $\delta$ s.

The results show that the learnt GBNs were capable of very high accuracy on average, ranging from 0.83 to 0.99 , but for some systems the minimum accuracy was low. We did not expect to have near perfect accuracy for all test data sets, so to measure the risk of generating a data set that the model will perform poorly on, we counted the number of times the accuracy was below $0.7,0.8$ and 0.9 . It is encouraging to see that there are not many cases where the accuracy is lower than 0.7 . Even in the case of system $\mathcal{S}_{4,3}$, which has 51 below 0.9 , there are only 18 below 0.8 and 14 below 0.7 . Furthermore, while there are some systems that have very low minimum accuracy, it seems that the number of samples that tend towards this worst case are few, e.g. $\mathcal{S}_{4,4}$ has the lowest minimum accuracy of 0.32 , but there are only 9 below 0.7 . 


\subsubsection{Conclusions}

From these synthetic experiments we conclude that our proposed algorithm performs well for both our primary and secondary aim (identification and detection). It is encouraging to see that the number of transition and the true transition structures were identified by the algorithm, and that the models performed well with respect to accuracy. A key feature of GBNs is that they are not mixture models, so each data point is not predicted as coming from a mixture of independence models, but from one distinct model. Taking into considerations the experiments using HMMs from Appendix $B$, we can clearly see that the mixture model does not perform as well as the GBNs that we learnt using our algorithm.

We shall now turn our attention to a real-world use of GBNs and regime identification. Specifically we shall look at how we can learn and use GBNs for describing the (potential) regimes in baseball players' career data.

\subsection{Regimes in baseball players' career data}

Baseball has been the de facto national sport of the United States since the late 19th century, and its popularity has spread to Central and South America as well as the Caribbean and East Asia. It has inspired numerous movies and other works of arts, and has also influenced the English language with expressions such as "hitting it out of the park" and "covering one's bases", as well as being the origin of the concept of a rain-check. As we all are aware, the omnipresent baseball cap is worn across the world. Excluding the postseason, there are approximately 2430 games of major league baseball in a season, equating to 162 games per team played over six months ${ }^{3}$ It speaks to the popularity of the game when the team with the lowest average attendance per game during the 2015 season had an average attendance of 21796 (the highest was 40 502).

Apart from being an extremely popular pastime, there is also a business side to baseball. A winning team tends to draw bigger crowds, thus ticket and merchandise sales increase. From a managerial perspective it is therefore necessary to analyse players to identify beneficial trades and strategies, in order to create the conditions for a successful team. Furthermore, with the growth of sports betting and fantasy leagues, this analysis is not only of interest for decisions makers within the teams, but from fans and spectators as well.

\footnotetext{
${ }^{3}$ While it may seem surprising, teams play virtually every day throughout the season, with only a few rest days scheduled.
} 
Bill James was the first to coin the term sabermetrics, referring to the empirical analysis of baseball statistics. SABR is an acronym for The Society for American Baseball Research, which was founded in 1971 and was a lynch pin for modern day baseball analysis, thus sabermetrics. Bill introduced the concept of ageing curves, a representation of how the ability of a certain player increases until it peaks at a certain age, and then falls off on the other side. This was an important insight, as it explained some of the variance in the summary statistics used at the time. Another of Bill's ideas was to compare current players with past players, creating a similarity score used to gain insight into how players performed relative to each other. It was later that Nate Silver combined these ideas to create the famous PECOTA system, which essentially used a nearest neighbour approach to create clusters of players based on their ageing curves [83]. While the system is used to create predictions of how players will perform over the coming season, it can also give insight into the worst-, best-, and most-likely scenario that will happen, as the ageing curves in the cluster of a player are also informative.

While owners and managers have always to some degree incorporated statistics as part of their strategy to trade players and win games, it was the release of Michael Lewis's book Moneyball [84] that unveiled to the general public the extent to which sabermetrics was used by Billy Beane when he managed the Oakland Athletics with great success, despite financial constraints. Today the use of sabermetrics is widespread across the league, but still creates headlines when teams like the Huston Astros employ former NASA engineers [85], or when breaches akin to corporate espionage are detected [86].

Naturally, one of the goals of sabermetrics is to produce predictions of players' future performance. There is, as one might suspect, a rich history of approaches for this task, and an untold number of books, magazines, news letters and forums are dedicated in furthering the performance of this endeavour [87, 88, 89]. From these efforts several well known systems have grown, such as Steamer [90], Marcel [91], and the aforementioned PECOTA system [87]. Interest from academics has also grown, including research on expected performance of both team's and player's [92, 93, 94]. However, sabermetricians are equally concerned with the process that leads to the prediction (Bill James' similarity scores and Nate Silver's outcome scenarios are examples of this). While we recognise the importance of making predictions, and acknowledge the difficulties in doing so, our intentions is in line with understanding the data. We are specifically interested in learning if there are regimes in the career data, and if so, how these regimes transition into one another. 
The reason why a baseball player would transition into a new regime may not be recorded or even directly observable, but may be a complex combination of increased skill and experience, ageing, strategic decisions, etc. For some regime changes it may be more evident what could have caused the shift in the player's performance, e.g. most of us would expect any sportsperson to play differently after an injury. What attracts our attention is the possibility of detecting regime transitions, and then identifying which of them are not directly explainable from publicly available data. The motivation for why this is of value lies in the fact that while coaches and managers make many decisions throughout a season, they may not be aware that a combination of these decisions may transition players to and from regimes in which they have been performing extraordinarily or subpar. Detecting regime changes in the data would allow decision makers to analyse the decisions they made during the time of the transition to see if they can recreate (or stay away from) the conditions that transitioned the player to the extraordinary (or subpar) regime.

\subsubsection{The game of baseball}

The official major league baseball rules are documented in a publication consisting of 282 pages, and is revised yearly for clarifications, removals and amendments. Here we will attempt to give only the most basic understanding of how the game of baseball is played, there are many more finer details and exceptions that we will not cover. We will on occasion refer to the stylised representation of a baseball field offered in Figure 4.5, the figure is an abstract representation and not to scale.

The game of baseball is played between two teams over nine innings, where in each inning the teams are given the opportunity to play both offense and defense. The team playing offense is able to score runs, and the team with the most runs at the end of the ninth inning is considered the winner. Extra innings are added if both teams have the same number of runs at the end of regulation play, there are no draws.

The main component of the game is a pitcher (positioned at 1 in the figure) that pitches (i.e. throws) the ball to the catcher (positioned at 2), both are on the defensive team. The batter, part of the offensive team, stands between the pitcher and catcher (at position 3 ) and tries to hit the ball. The remaining defensive players are positioned strategically on the field. The defensive team needs to get three batters out in order to end their defensive part of the inning.

There are several outcomes that may take place when the ball is pitched, we describe the relevant outcomes here: 


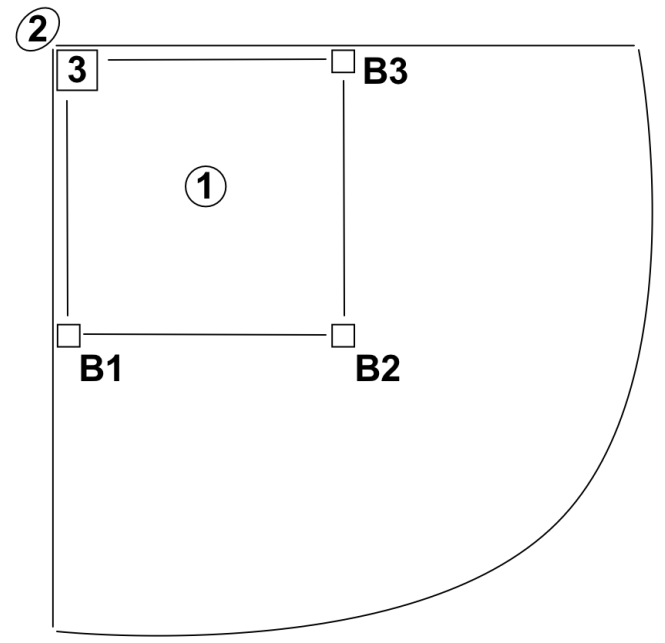

Figure 4.5: Stylised representation of a baseball field.

- Assuming that the batter does not swing the bat at all, a ball that is pitched to the catcher can count as either a strike or a ball. If the ball passes the batter within the strike zone then the pitch is counted as a strike, if it is outside the strike zone then it is counted as a ball. The strike zone is an imaginary volume of space that extends from the batters knees to the midpoint of the batters torso. Simply put, it is a zone in which the batter has a fair chance of hitting the ball. Whether or not the ball is within the strike zone is decided by an umpire positioned behind the catcher.

- If the batter swings at the ball and misses then it counts as a strike, even if the pitch was outside the strike zone.

- If the strike count goes to three, then the batter is out. If the ball count goes to four, then the batter can freely move to first base (positioned at B1 in the figure), this is known as a walk.

- If the batter hits the ball then there are two main outcomes. The ball can either fly into the air and be caught by a defensive player before hitting the ground, at which point the batter is out. Alternatively, the ball can hit the ground in which case the batter becomes a runner, and needs to reach first base before a player in the defensive team touches first base while holding the ball. The runner (previously known as the batter) is also allowed to attempt to advance to second or third base (2B 
and $3 \mathrm{~B}$ in the figure), but will be out if a player on the defensive team manages to touch the runner while holding the ball. If caught then the runner is out, if safe at a base then the play is over and the next player in the offensive team becomes the batter. Each time an offensive player is allowed an attempt to bat it is counted as a plate appearance. If there was an offensive player already on a base when the batter hit the ball, then they can advance further (sometimes they are forced to advance).

- Each time the offensive team gets a runner all the way around the bases, i.e. running through B1, B2, B3 and back to where the batter is positioned at 3 in the figure, then the offensive team scores a run.

- If the batter hits the ball outside the field, e.g. into the stands or even outside the arena itself, then it is called a home run and the batter can freely run through all bases and come back home, thereby scoring a run. Any other offensive players that were already safe at one of the bases can also run home, thus scoring a run for each player that comes home.

- There are several other reasons why a batter may freely move to first base, for instance if the pitcher hits the batter with the ball or the defensive team makes a rule error.

There is a myriad of statistics developed that aim at summarising a player's performance. We will use a statistic developed by Bill James, known as the somewhat cumbersome on-base plus slugging (OPS) statistic which is calculated by Equation 4.13 .

$$
\begin{aligned}
& S L G=\frac{\text { single }+2 * \text { double }+3 * \text { triple }+4 * \text { home run }}{\text { at-bat }} \\
& O B P=\frac{\text { hit }+ \text { walk }+ \text { hit by pitcher }}{\text { at-bat }+ \text { walk }+ \text { sacrifice fly }+ \text { hit by pitcher }} \\
& O P S=O B P+S L G
\end{aligned}
$$

When a batter hits the ball and makes it to first base, it counts as a single, if the batter makes it to second base it is called a double, a triple represents making it to third base and home runs are home runs. As is evident from the calculation of slugging (SLG) there is a weighting on how much the outcomes are worth, and the linear combination is divided by the number of at-bats. While the number of plate appearances does not equate exactly to the number 
Table 4.4: Discretisation of OPS statistics.

\begin{tabular}{|l|l|l|}
\hline Label & Description & OPS range \\
\hline A & Great & {$[0.9000,+\infty)$} \\
B & Very good & {$[0.8333,0.9000)$} \\
C & Above average & {$[0.7666,0.8333)$} \\
D & Average & {$[0.7000,0.7666)$} \\
E & Below average & {$[0.6333,0.7000)$} \\
F & Poor & {$[0.5666,0.6333)$} \\
G & Atrocious & {$[0,0.5666)$} \\
\hline
\end{tabular}

of at-bats, it can be thought of as the same for the purposes of understanding our experiments. If a player hits either a single, double, triple or home run it counts as a hit, which is used in the calculation of on-base percentage (OBP). A walk is as described earlier, and hit by pitcher happens when the pitcher hits the batter with the ball. A sacrifice fly is a strategic option where the batter on purpose hits the ball far and high, making it easy for the defensive team to catch it before it hits the ground thus giving them an out, but an offensive player safe on a base may make it back to score a run (thus the batter is sacrifised). The OPS statistic aims to represent an offensive player's skill, however does not take into account the player's running abilities (fast players are not credited by an increased OPS). Bill James also suggested a discretisation of OPS, presented in Table 4.4 .

Apart from OPS we will also make use of the less complex runners batted in (RBI) statistic, which is a count of how many runs a batter is responsible for, e.g. if the offensive team has one runner safe on third base and the batter hits the ball allowing the runner to make it back home, then it counts as a run for the offensive team and a RBI for the batter.

Following the above definitions, we here present the variables that we extracted for our purposes:

- Outcome - The outcome of the event, with states: single, double, triple, home-run, walk, out or reaching first base for another reason.

- OPS - A 30 day rolling window calculation of OPS, discretised according to Table 4.4 .

- RBI - A 30 day rolling window calculation of RBI, discretised into three equal width bins.

- B1 - Whether or not there is a runner on first base (true or false).

- B2 - Whether or not there is a runner on second base (true or false).

- B3 - Whether or not there is a runner on third base (true or false). 
- Home - Whether or not the offensive team is the home team (true or false).

- Arm - Whether or not the pitcher throws with the left or right arm (left or right).

- Outs - The number of outs in the inning $(0,1$ or 2$)$.

- Balls - The ball count $(0,1,2$ or 3$)$,

- Strikes - The strike count $(0,1$ or 2$)$.

\subsubsection{Setup of experiments}

We shall account for two experiments, one on real-world data and one on synthetic data (for this set of synthetic data we generate career data for fictive baseball players). As we did for the experiments described in Section 4.4, we also ran the synthetic experiments using HMMs. The outcome of the HMM experiments can be found in Appendix C.

\section{Synthetic data}

We created one BN, using the variables introduced in Section 4.5.1, and then manipulated this $\mathrm{BN}$ slightly to create another $\mathrm{BN}$, representing a new regime. We continued this manipulation until we had five different BNs, representing five different regimes in a fictional baseball player's career. The five BNs created are shown in Figure 4.6. We then arranged these BNs into three different regime transition structures, depicted in Figure 4.7, and sampled data sets from these structures. For each structure, one data set was used for learning, and 100 data sets were held out for testing. The data sets were created by sampling a random number of samples (uniform between 250 and 600) from each regime, and then concatenating to create a data set. For instance, for Structure 2 in Figure 4.7, we first sampled a random number of samples from $R_{1}$ in Figure 4.6, then another random number of samples from $R_{2}$, then from $R_{1}$ again, and finally from $R_{3}$ and $R_{4}$. The testing data was used to estimate the accuracy of the learnt GBN, i.e. measuring whether or not the GBN had the correct BN activated given the testing data, as described in Section 4.2.3.

\section{Real-world data}

Retrosheet is an organisation founded in 1989 [95] that digitally stores events of major league baseball games. Each event describes the outcome of a plate 

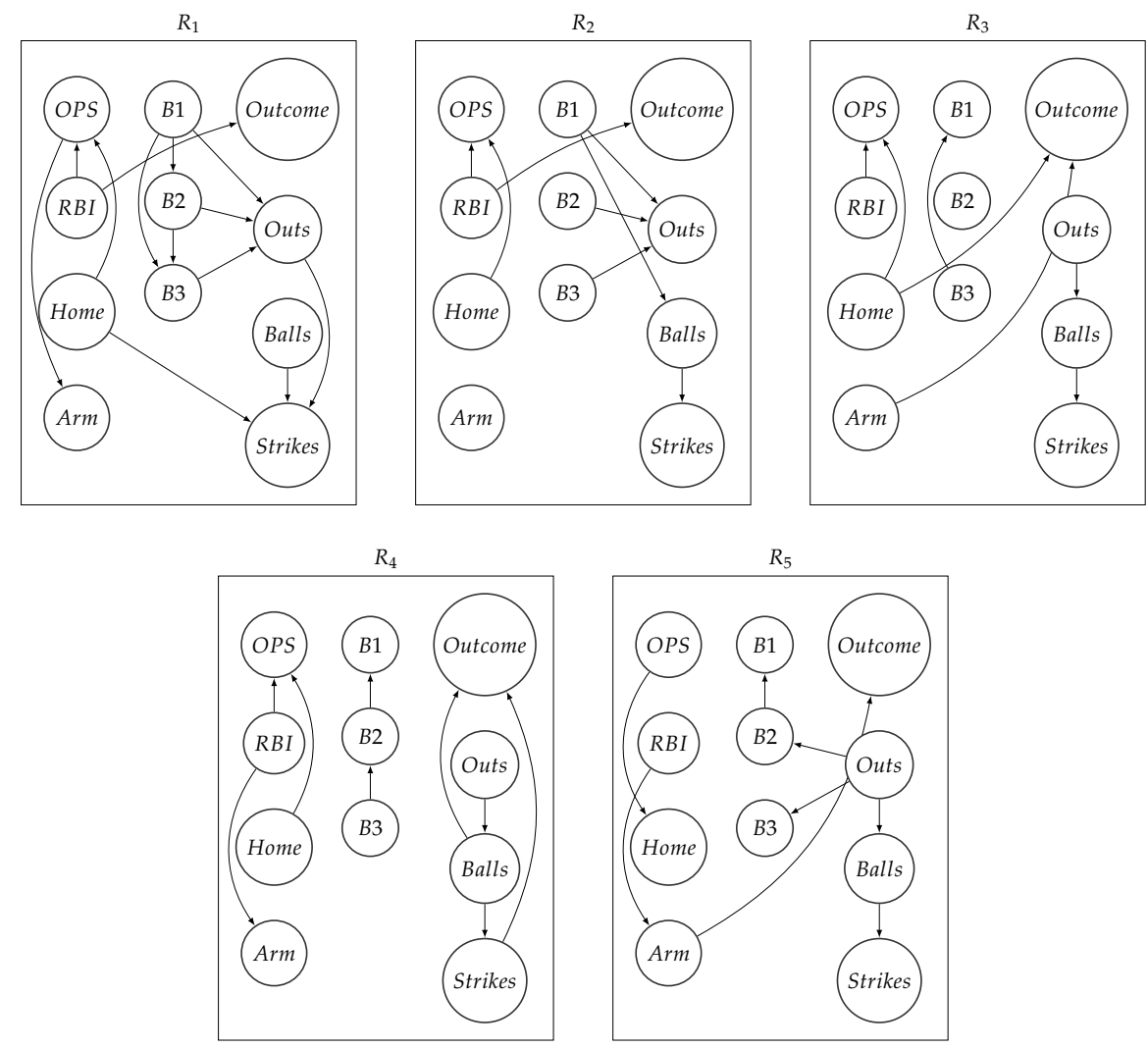

Figure 4.6: BNs created to generate synthetic career data.

appearance, and the conditions under which the event happened. There are over 8900 players in the Retrosheet database, for which a complete analysis was not within our aims. Instead we defined a subpopulation of all these players, and then sampled from this subpopulation. We filtered the event data in such a way that we were left with players that had their major league debut during the 2005 season or later, and with the criteria that they had to have at least 2000 event data entries. The range of number of events in this subpopulation was 2001 to 6614 . This subpopulation consisted of 150 players, from which we uniformly sampled 30 players.

\subsubsection{Methodology}

During MH we used $k=8$ splits, thus we are not assuming any knowledge about how many splits there truly are in the data, only that there are a maximum of eight. We ran 100000 iterations, throwing away the first 50 000 samples as burn-in. The greedy thick-thinning algorithm introduced in 
Structure 1

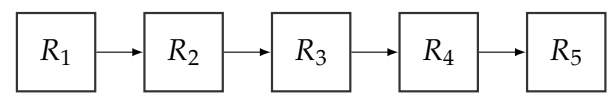

Structure 2

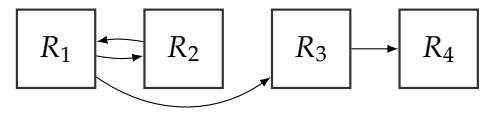

Structure 3

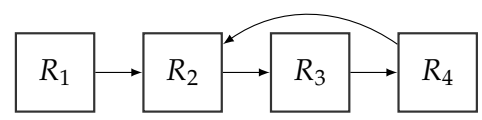

Figure 4.7: Transition structures used in synthetic experiments.

Section 2.1.3 was used, where the log marginal likelihood was the target to improve. We ran 250 iterations of Bayesian optimisation using the radial basis kernel with $c=2.0$, and the upper confidence bound criterion acquisition function using $\eta=5$. The objective function was the average accuracy over 1000 resamples.

\section{Synthetic data}

For the synthetic data we used the generated learning data sets to learn a GBN for each transition structure, and then used the 100 testing data sets to compute the accuracy of the learnt GBNs. We shall report the location of the identified regime transitions, and compare them with the true locations, as well as the average accuracy of the GBNs over the test data sets.

\section{Real-world data}

In order to count the number of regime transitions for which there is an evident cause, we created the following three criteria. If a regime transitioned happened in conjunction with an event for which any of the criteria are satisfied, then the cause was considered evident:

1. An injury to the player - We expect a player to play differently a period after an injury, thus if a regime transition coincides with an injury we count it as a transition where the cause is known 4

2. The player being traded - When a player moves to a new team there is a good chance that the player needs to learn a new system, new coaching and may need time to adjust. While the trade itself may not be

\footnotetext{
${ }^{4}$ Information about players' injuries and trades were sourced from their respective Wikipedia pages.
} 
the cause, a transition in conjunction with a trade is to some degree explained by the trade. ${ }^{4}$

3. The beginning of a new season - A new season usually brings new players to a team, sometimes new managers and coaches, new strategies are put in place, etc. A regime transition early in the season is therefore considered to be explained by these changes.

From these criteria it should be understood that transitions not counted towards the evident reasons are those where a transition occurs mid season, without a major injury or trade. The reason for such a transition may be clear for the managers and coaches, since they have access to data that the public has not, however they may also be unaware of these transitions, and identifying the transitions allows for analysis of what may have caused them. It is precisely here that our procedure adds value to the decision making by managers and coaches, discovering transitions that may have been unintentional or the result of multiple events and decisions.

\subsubsection{Results and discussion}

We will divide this section into four parts. In the first part we will account for the performance of the GBN learning using the synthetic data sets. In the second part we will give a summary view of the GBNs that were learnt for the 30 sampled players, and then immediately discuss our findings in relation to this sample. Thereafter we will look at two players in more detail. This detailed analysis allows us to show the GBNs that were learnt, as well as discuss the regime transitions which we can explain and which we cannot. Finally, we will end this section with a discussion regarding the representational power of GBNs versus BNs.

\section{Synthetic data}

In Table 4.5 we present the true regime transition locations in the synthetic data, the locations identified using the proposed learning algorithm, and the difference between the two. The three structures are the ones presented in Figure 4.7. As is evident from the table, the proposed learning algorithm identifies well the true locations, as the differences are relatively small. The merging procedure of the learning algorithm was also able to correctly identify the regime transition structures in all three cases. As was the case with the first set of synthetic experiments, the HMM does not perform as well as the GBN at this task, as is evident from the results in Appendix C 
Table 4.5: Location of identified regime transitions during GBN learning using synthetic data.

\begin{tabular}{|l|llll|llll|llll|}
\hline & \multicolumn{4}{|c|}{ True locations } & \multicolumn{4}{|c|}{ Identified locations } & \multicolumn{4}{c|}{ Difference } \\
\hline Structure 1 & 590 & 977 & 1360 & 1933 & 574 & 990 & 1337 & 1931 & 16 & 13 & 23 & 2 \\
\hline Structure 2 & 364 & 823 & 1321 & 1683 & 355 & 809 & 1322 & 1711 & 9 & 14 & 1 & 28 \\
\hline Structure 3 & 351 & 884 & 1223 & 1757 & 337 & 886 & 1212 & 1743 & 14 & 2 & 11 & 14 \\
\hline
\end{tabular}

Table 4.6: Accuracy of learnt GBNs using synthetic data.

\begin{tabular}{|l|c|c|c|c|c|c|}
\hline & $\tau$ & $\theta$ & Average & Minimum & $\#<0.9$ & $\#<0.8$ \\
\hline Structure 1 & 10 & 1.08 & 0.98 & 0.98 & 0 & 0 \\
\hline Structure 2 & 13 & 1.18 & 0.94 & 0.77 & 14 & 1 \\
\hline Structure 3 & 10 & 1.07 & 0.97 & 0.82 & 7 & 0 \\
\hline
\end{tabular}

Turning our attention to Table 4.6, we present the optimised $\tau$ and $\theta$ values for each structure, along with the average and minimum accuracy achieved over the 100 test data sets. As is evident, the average accuracy is high, however since the minimum for Structure 2 was lower, we also counted the number of times the accuracy was below 0.9 and 0.8 . From the table we can tell that such occurrences were few.

All in all, these results show that the proposed learning algorithm is capable of correctly identifying regime transition structures, and that the resulting GBN can be used to accurately identify the current regime, even when the number of variables, and the number of states these variables take, increase (compared to the synthetic experiments described in Section 4.4).

\section{Summary view}

For each baseball player analysed we present a summary of the GBN learnt in Table 4.7. In the table we first present the player's name, followed by the number of nonzero $\delta$ s identified, i.e. the number of transitions. The table continues with the number of BNs contained in the GBN, and a Boolean value representing whether or not there were reoccurring regimes in the GBN, i.e. if the GBN contains directed cycles. The fifth column contains the number of regime transitions for which we could find an evident cause, and the sixth column the number for which we could not. The last two columns are the $\tau$ and $\theta$ values determined during optimisation of the gate parameters.

The first observation we make is the fact that there certainly are regimes in the data, using a single BN to represent a player's career is not preferred for any of the players. What is further interesting is that, for all but two players, the regimes are not structured in a chain, but the regimes rather reoccur. While we expect any sportsperson to change the way they play their 
Table 4.7: Summary view of the GBNs learnt for a sample of baseball players.

\begin{tabular}{|l|c|c|c|c|c|c|c|}
\hline Player & $\delta \mathrm{s} \neq 0$ & BNs & Cycles & Explained & Unexplained & $\tau$ & $\theta$ \\
\hline Ike Davis & 5 & 4 & $\mathrm{~T}$ & 2 & 3 & 26 & 1.10 \\
\hline Nate McLouth & 5 & 4 & $\mathrm{~T}$ & 2 & 3 & 18 & 1.04 \\
\hline Chris Denorfia & 3 & 3 & $\mathrm{~T}$ & 1 & 2 & 19 & 1.07 \\
\hline Jose Altuve & 5 & 5 & $\mathrm{~T}$ & 2 & 3 & 10 & 1.13 \\
\hline Ian Desmond & 4 & 3 & $\mathrm{~T}$ & 1 & 3 & 17 & 1.11 \\
\hline Desmond Jennings & 6 & 5 & $\mathrm{~T}$ & 1 & 5 & 16 & 1.08 \\
\hline Nyjer Morgan & 6 & 4 & $\mathrm{~T}$ & 3 & 3 & 15 & 1.15 \\
\hline Matt Wieters & 3 & 3 & $\mathrm{~T}$ & 1 & 3 & 32 & 1.05 \\
\hline Kendrys Morales & 5 & 4 & $\mathrm{~T}$ & 2 & 3 & 45 & 1.02 \\
\hline Melky Cabrera & 6 & 5 & $\mathrm{~T}$ & 4 & 2 & 50 & 1.02 \\
\hline Carlos Quentin & 5 & 4 & $\mathrm{~T}$ & 4 & 1 & 47 & 1.01 \\
\hline Freddie Freeman & 5 & 4 & $\mathrm{~T}$ & 1 & 4 & 11 & 1.08 \\
\hline Nick Hundley & 3 & 3 & $\mathrm{~T}$ & 2 & 1 & 24 & 1.04 \\
\hline Alberto Callaspo & 5 & 4 & $\mathrm{~T}$ & 2 & 3 & 17 & 1.03 \\
\hline Jason Kipnis & 5 & 4 & $\mathrm{~T}$ & 1 & 4 & 11 & 1.08 \\
\hline Buster Posey & 4 & 4 & $\mathrm{~T}$ & 1 & 3 & 43 & 1.04 \\
\hline Ryan Doumit & 3 & 4 & $\mathrm{~F}$ & 1 & 2 & 27 & 1.06 \\
\hline Jason Heyward & 6 & 4 & $\mathrm{~T}$ & 3 & 3 & 10 & 1.07 \\
\hline Michael Brantley & 3 & 4 & $\mathrm{~F}$ & 2 & 1 & 10 & 1.14 \\
\hline Troy Tulowitzki & 4 & 3 & $\mathrm{~T}$ & 3 & 1 & 25 & 1.02 \\
\hline Kyle Seager & 5 & 5 & $\mathrm{~T}$ & 1 & 4 & 10 & 1.10 \\
\hline Mark Teahen & 5 & 5 & $\mathrm{~T}$ & 2 & 3 & 37 & 1.85 \\
\hline Will Venable & 4 & 3 & $\mathrm{~T}$ & 1 & 3 & 10 & 1.06 \\
\hline Daric Barton & 4 & 4 & $\mathrm{~T}$ & 1 & 3 & 18 & 1.09 \\
\hline Gerardo Parra & 4 & 4 & $\mathrm{~T}$ & 1 & 3 & 10 & 1.08 \\
\hline Gregor Blanco & 4 & 4 & $\mathrm{~T}$ & 1 & 3 & 15 & 1.07 \\
\hline Rajai Davis & 2 & 2 & $\mathrm{~T}$ & 1 & 1 & 10 & 1.06 \\
\hline Alex Avila & 4 & 4 & $\mathrm{~T}$ & 2 & 2 & 43 & 1.00 \\
\hline Adam Lind & 5 & 4 & $\mathrm{~T}$ & 4 & 1 & 45 & 1.06 \\
\hline Kevin Kouzmanoff & 3 & 3 & $\mathrm{~T}$ & 1 & 2 & 16 & 1.04 \\
\hline
\end{tabular}

respective game throughout their career, be it due to increasing skill or age, it is not necessarily obvious why we find that some players revert back to previous regimes. Though highly speculative at this point, transitioning into one regime and then transitioning back may be due to strategic decisions by the manager or coach, or due to injury, since players may be playing more cautiously while receiving treatment.

There certainly is an overweight towards regime transitions which we could not explain from our superficial investigation. While we are not ruling out that there may be publicly available sources of information that may be helpful in order to explain the remaining transitions, it is noteworthy that there is reason for further investigation. As we shall see in the detailed analysis, some of these transitions may be highly sought after (e.g. transitioning into a regime where a player is playing extraordinarily well), thus for the managers and coaches it can be essential to look at the decisions that were made at the time of the transition. 
The last thing we wish to comment on regarding this summary view are the $\tau$ and $\theta$ values. In the synthetic experiments reported in Table 4.6 we saw relatively low values for $\tau$ in all three cases. However, for the synthetic experiments reported in Table 4.3 the algorithm produced GBNs with high accuracy and the average $\tau$ value was 21.2 (with a range of [10,50]). As is evident from the last two columns in Table 4.7, the $\tau$ values for the GBNs learnt in these experiments are comparable with the synthetic experiments. What this tells us is, that if the GBNs were to be used on new data, for instance the 2017 season, they would not require an unusually large window $(\tau)$ to detect regime changes, and the required threshold $(\theta)$ is comparable to what we have shown had good accuracy on synthetic data. An unusually large window could imply that the GBN would be detecting regime changes much later than they occurred, thus the use of the GBN as a detection model would have been limited. So while we do not explore this opportunity further here, the learnt GBN is a model that can be used to detect if the player has transitioned to any of the identified regimes. We imagine that a coach could learn a GBN using data from previous seasons, from this GBN identify what the causes were for a favourable regime transition, put these causes in place and then monitor the player by entering the new data that the player is generating into the GBN. If the GBN does transition into the intended regime, then the coach has succeeded.

\section{Detailed analysis}

In order to gain further understanding of how the regime changes in the players' data can be used, we picked out two players that served as good examples for how a manager or coach could do a further analysis. For these players, Nyjer Morgan and Kendrys Morales, we present the GBNs learnt in Figure 4.8 and Figure 4.10. In Figure 4.9 and Figure 4.11 we present representations of the subsetting of the data, similar to the ones given in the example in Figure 4.2. In these representations we have marked to which regime each subset belongs, as well as the OPS statistic and label according to the discretisation in Table 4.4 for each particular subset. We have also added the date on which the transitions occurred.

We will begin our analysis with Nyjer Morgan (Figure 4.8 and Figure 4.9). We first remark on the OPS labels given to the regimes. The subsets 2, 4 and 7 all belong to regime $R_{2}$, which have the OPS labels below average, poor and below average, thus we conclude that the $R_{2}$ regime represents a low performance regime for Nyjer (with respect to OPS). The subsets 1 and 3 belong to $R_{1}$ and have been given average and above average, and we will 
therefore refer to regime $R_{1}$ as a high performance regime. Subset number 5 is the only subset that belongs to regime $R_{3}$ with the label above average, a regime that also represents high performance, but not the same as $R_{1}$. Subset number 6 is the sole subset for regime $R_{4}$, and given the OPS label above average it also seems to correspond to a high level of performance, however we note that the OPS value of 0.775 in subset 6 is lower compared to 0.818 in subset 5 .

Turning our attention to the regime transitions, we will offer a narrative that may explain why the transitions happened at these specific dates. Nyjer's major league career started with the Pittsburgh Pirates, where he was given the starting role in left field in the beginning of the 2009 season (this was based on a positive second half of 2008). We see a transition from the high performance regime $R_{1}$ to the low performance regime $R_{2}$ on the 6th of April (the 2009 season started on the 5th of April, but the Pirates first game was on the 6th). At the beginning of any season there are a lot of changes to the team and tactics, including giving Nyjer the starting role in left field. Perhaps due to the performance of Nyjer, he was traded on the 30th of June 2009 to the Washington Nationals. We see a regime change on the 23rd of June to the high performance regime $R_{1}$ at approximately the same time. The next regime change on the 2 nd of May is however not as easy to pinpoint. It is another move from $R_{1}$ to $R_{2}$ (high to low performance), which is not necessarily tied to a new season, trade or injury. However, Nyjer was involved in some headline worthy mistakes and unsportsmanlike actions during the second half of 2010, that may have affected the way he played. In any case, this is a type of transition where a manager or coach could delve deeper into their data to find the causes of this regime transition. On the 27th of March 2011 Nyjer was traded to the Milwaukee Brewers (this happened during the offseason), and in the beginning of the 2011 season, on the 6th of April 2011, a new regime change occurred (Milwaukees first game of the 2011 season was on the 31st of March, but Nyjer did not play full games the first days of the season). During this last transition Nyjer moved into $R_{3}$, a high performing regime which lasts until the 23rd of July 2011, when Nyjer transitions to $R_{4}$ (also a high performance regime, but as we noted with a lower OPS), and then to the low performance regime $R_{1}$ on the 19th of August 2011. These last two regimes cannot be directly accredited to a new season, trade or injury, and are examples of transitions needing further investigation by managers and coaches. What can be said is that the regime transitions $R_{3}$ to $R_{4}$ and finally $R_{2}$ seem to indicate a dwindling performance for Nyjer, with respect to the OPS values. He would later take contracts for other major league teams, as 


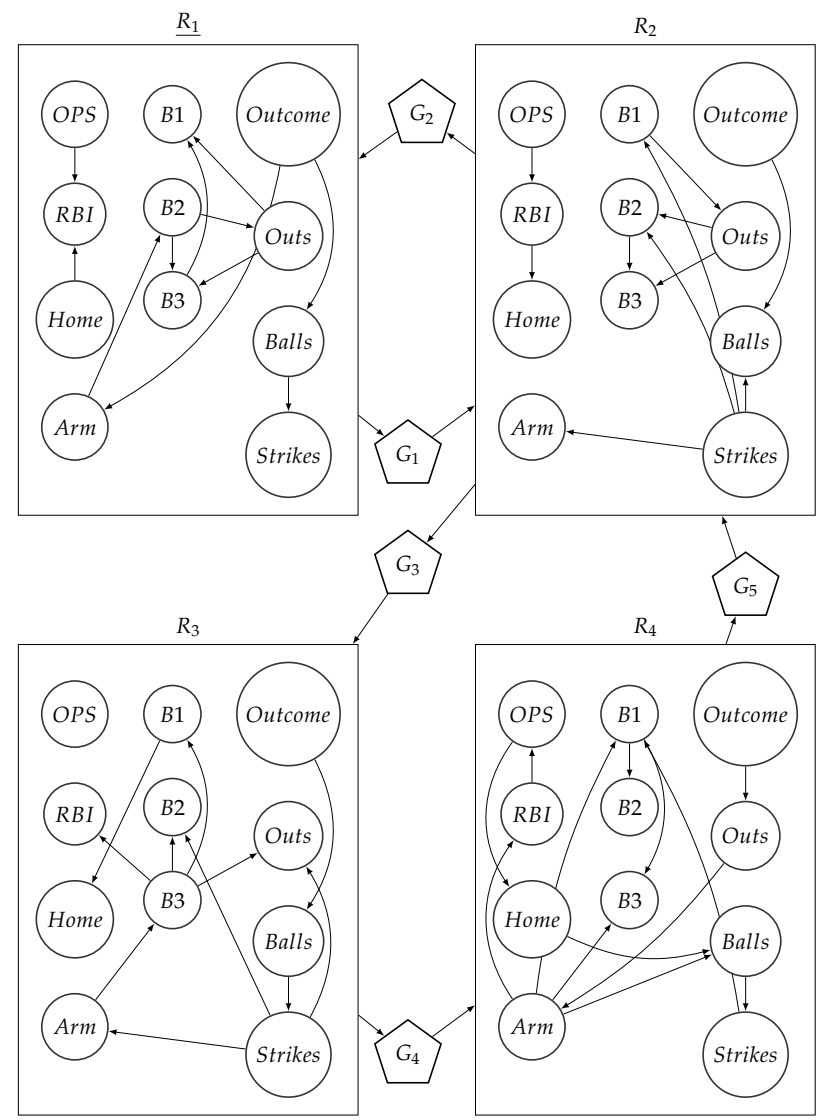

Figure 4.8: GBN learnt for Nyjer Morgan.

\begin{tabular}{|c|c|c|c|c|c|c|}
\hline $\mathrm{R}_{1}$ & $\mathrm{R}_{2}$ & $\mathrm{R}_{1}$ & $\mathrm{R}_{2}$ & $\mathrm{R}_{3}$ & $\mathrm{R}_{4}$ & $\mathrm{R}_{2}$ \\
\hline 0.738 & 0.695 & 0.820 & 0.616 & 0.818 & 0.775 & 0.645 \\
\hline 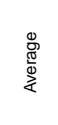 & 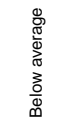 & 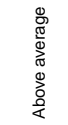 & ¿̀ & 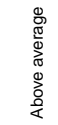 & 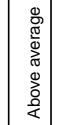 & 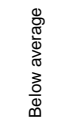 \\
\hline
\end{tabular}

Figure 4.9: Regime subsets with OPS statistic and label for Nyjer Morgan. 


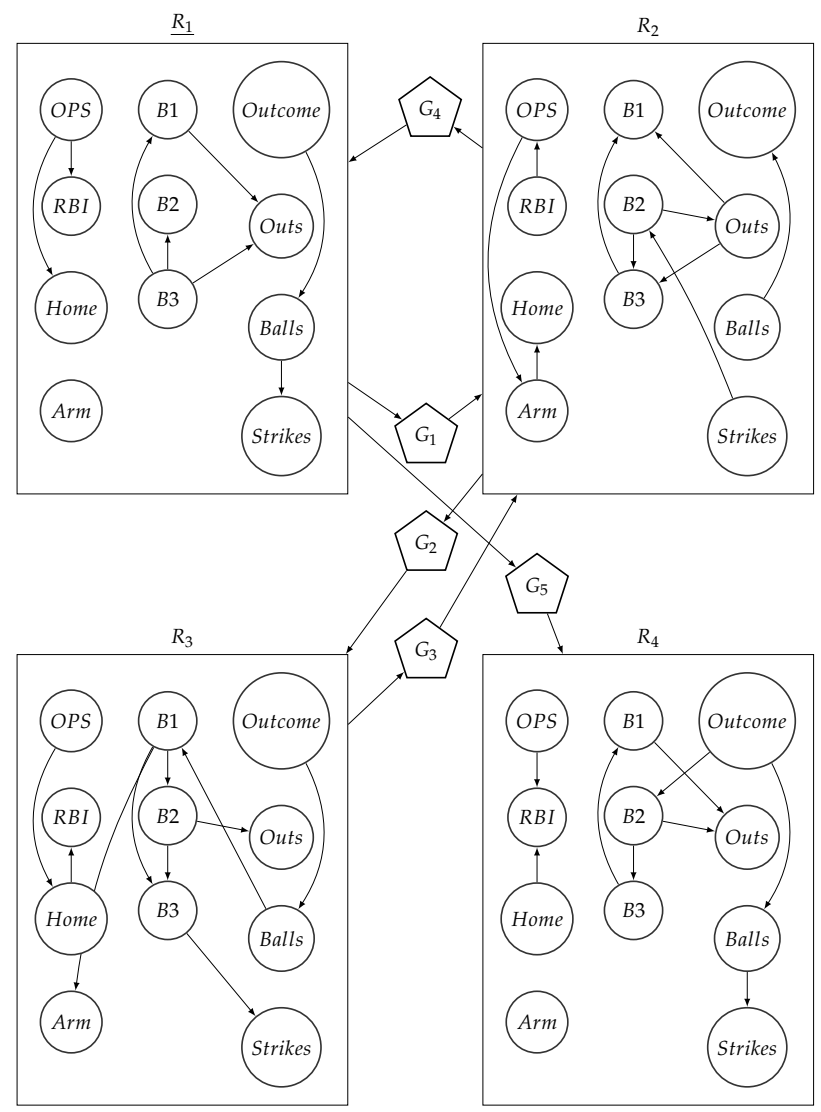

Figure 4.10: GBN learnt for Kendrys Morales.

\begin{tabular}{|c|c|c|c|c|c|}
\hline $\mathrm{R}_{1}$ & $\mathrm{R}_{2}$ & $\mathrm{R}_{3}$ & $\mathrm{R}_{2}$ & $\mathrm{R}_{1}$ & $\mathrm{R}_{4}$ \\
\hline 0.732 & 0.755 & 1.053 & 0.819 & 0.817 & 0.631 \\
\hline 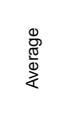 & 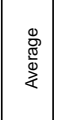 & $\begin{array}{l}\mathbb{\Xi} \\
\mathbb{\Phi} \\
\bar{J}\end{array}$ & 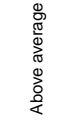 & 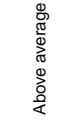 & ๖̀ \\
\hline
\end{tabular}

Figure 4.11: Regime subsets with OPS statistic and label for Kendrys Morales. 
well as stints in both Japan and Korea. However Nyjer has not returned to his former high level performance $R_{1}$ or $R_{3}$ regimes.

The second player we will analyse is Kendrys Morales, for whom we display the GBN and subset representation in Figure 4.10 and Figure 4.11. For Kendrys we could just as well assign a high performance label to both $R_{1}$ and $R_{2}$ as they have one subset with average and above average respectively. The narrative we soon will offer may clear up the distinction, however it serves as a reminder that summary statistics such as OPS does not tell the full story. Regime $R_{3}$ represents exceptional performance, as the subset has been given the label great. The last regime $R_{4}$ will be referred to as a low performance regime due to the OPS label poor.

As we did for Nyjer, we will offer an explanation for the transitions we found in Kendrys' data. At the beginning of the 2009 season Kendrys was promoted to starting first baseman 5 . He had a fantastic season, playing well in the beginning and even better in the second half of the season. He was given the Player of the Month award in August 2009. The first transition on the 14th of May from $R_{1}$ to $R_{2}$ came early in the season (the 2009 season started on the 5th of April), and while the transition is not exactly on the first day of the season, it is reasonable to assume that Kendrys was adjusting into his new position. There is no evident reason for the transition from $R_{2}$ to $R_{3}$ on the 17 th of June, however it is clear that Kendrys was playing better, thus there is certainly reason for further investigation into why there was a transition on this date. The transition from $R_{3}$ to $R_{2}$ happened on the 7 th of September 2009, again a transition for which no evident reason can be given. However, looking at the OPS values rather than the labels, we see that the transitions $R_{1} \rightarrow R_{2} \rightarrow R_{3} \rightarrow R_{2}$ coincide with an increase, peak and decline of the OPS value. A question, to which we have no direct answer, is to ask whether this is simply due to Kendrys going on a hot-streak and then cooling off, or if there were actions taken at these times that caused the transitions through these regimes. If the latter is the answer to the question, then it would be extremely valuable for managers and coaches to investigate these regime changes in order to find these causes so that they may be put in place again. In an unfortunate turn of events, Kendrys was injured on the 29th of May 2010 (coinciding with the transition from $R_{2}$ to $R_{1}$ ). He was sidelined for the rest of the 2010 season and the entire 2011 season. In 2012 Kendrys came back, and it seems that he was able to play at a reasonable level as before $\left(R_{1}\right)$,

\footnotetext{
${ }^{5}$ Baseball players usually occupy a specific position on the field when playing defense, thus players are often referenced to as first baseman, second baseman, shortstop, etc. However, these players still bat and play offense, but since everybody is either a batter or runner when playing offense it is the defensive position that is referenced.
} 
Table 4.8: Log marginal likelihood of data subsets given individual BNs (Nyjer Morgan).

\begin{tabular}{|l|l|l|l|l|l|l|l|}
\hline & Subset 1 & Subset 2 & Subset 3 & Subset 4 & Subset 5 & Subset 6 & Subset 7 \\
\hline$R_{1}$ & $\mathbf{- 2 4 1 6 . 1 6}$ & -2763.77 & $\mathbf{- 3 2 2 8 . 2 6}$ & -4252.85 & -2007.11 & -797.71 & -4693.33 \\
\hline$R_{2}$ & -2427.00 & $\mathbf{- 2 7 5 6 . 2 0}$ & -3236.63 & $\mathbf{- 4 2 5 1 . 7 2}$ & -2007.46 & -801.51 & $\mathbf{- 4 6 8 9 . 5 8}$ \\
\hline$R_{3}$ & -2437.66 & -2856.39 & -3288.64 & -4304.85 & $\mathbf{- 1 9 8 7 . 9 4}$ & -819.07 & -4719.25 \\
\hline$R_{4}$ & -2464.09 & -2831.43 & -3348.70 & -4355.55 & -2027.88 & $\mathbf{- 7 7 3 . 2 1}$ & -4791.58 \\
\hline \hline Single & -2446.08 & -2770.41 & -3238.49 & -4270.29 & -2019.44 & -809.21 & -4698.99 \\
\hline
\end{tabular}

Table 4.9: Log marginal likelihood of data subsets given individual BNs (Kendrys Morales).

\begin{tabular}{|l|l|l|l|l|l|l|}
\hline & Subset 1 & Subset 2 & Subset 3 & Subset 4 & Subset 5 & Subset 6 \\
\hline$R_{1}$ & $\mathbf{- 4 9 2 9 . 3 8}$ & -1266.78 & -2690.71 & -3206.58 & $\mathbf{- 9 8 9 7 . 5 8}$ & -5472.86 \\
\hline$R_{2}$ & -4945.14 & $\mathbf{- 1 2 5 7 . 9 5}$ & -2696.66 & $\mathbf{- 3 2 0 0 . 2 9}$ & -9945.11 & -5487.72 \\
\hline$R_{3}$ & -5027.57 & -1266.08 & $\mathbf{- 2 6 6 7 . 8 4}$ & -3258.13 & -10078.60 & -5532.56 \\
\hline$R_{4}$ & -4931.26 & -1260.78 & -2695.41 & -3217.07 & -9900.53 & $\mathbf{- 5 4 6 0 . 9 0}$ \\
\hline \hline Single & -4959.28 & -1285.23 & -2730.32 & -3235.33 & -9943.27 & -5496.74 \\
\hline
\end{tabular}

but did not come back to his high and extraordinary performance regimes $R_{2}$ and $R_{3}$. Kendrys was traded to the Seattle Mariners prior to the 2013 season, however the transition from $R_{1}$ to $R_{4}$, the low performance regime, did not come until mid season. While Kendrys was traded, the data does not suggest that this change caused any disruption in Kendrys performance, but rather something that happened later.

\section{Gated Bayesian networks versus Bayesian networks}

From a modelling perspective, regardless of application domain, it is interesting to note that the BNs that are learnt do in fact represent the data better for their subsets than do the other BNs. This is shown in Table 4.8 and Table 4.9. where the log marginal likelihood for each combination of subset and $\mathrm{BN}$ is presented, as well as for a single BN that was learnt using all the data (using data for Nyjer Morgan and Kendrys Morales). Note that these are log-terms, so differences may look small in some cases when they are in fact orders of magnitude.

It is clear then that if we use a single BN to represent our data, we will not be able to capture and take advantage of the structural changes in the data. However, while treating a weakness of BNs we are also exposing one of GBNs. Looking at Figure 4.8 and Figure 4.10 it is definitely much harder to now grasp what the underlying structure of the data is. What we gain in expressive power, we lose in clarity. On the positive side, if one takes the time to analyse the BN structures in the GBNs, valuable pieces of information 
may be extracted. For instance, in Figure 4.10 we see that only in $R_{2}$ does the pitcher's throwing arm stand in relation with Kendrys playing at home and his recent OPS value. This may be due to the opposing teams' coaches choosing a favourable matchup (it is generally accepted that a right handed pitcher has an advantage over a right handed batter, and the same goes for left on left, however the batter has the advantage when they are opposite). Adding to the complexity is the fact that Kendrys himself is a switch hitter, meaning that he will change his batting hand to always have the advantage. Perhaps this is why the relationships are only brief, the opposing coaches giving up on trying this tactic. Whatever the reason for these brief relationships, they would vanish if one learnt a single BN using all the data, thus this type of extraction would not be possible.

\subsubsection{Summary}

All sports seem to demand a certain degree of data collection, it would be hard to imagine any type of competitive market for trading players in any sport without some statistical description of the players. For baseball however, it seems to have been taken to a level of healthy obsession. Retrosheet is certainly a remarkable endeavour, not only attempting, but doing very well in achieving its goal to computerise every single plate appearance of every single major league game since 1871 . We have only picked out a handful of the variables that are represented in the database. It is however possible that this obsession also creates a certain bias, as those looking at the data may become falsely assured that usable information is extractable simply due to the sheer amount of data available. Nonetheless, the penetration of sabermetrics into major league teams is undeniable.

We have investigated the use of GBNs for modelling baseball players' careers, specifically in order to identify regimes in the data. It is clear from our experiments that such regimes do exist, and that these regimes can reoccur. We have also shown how a deeper analysis of a GBN allows us to label the regimes depending on the player's performance, thereby offering a tool to managers and coaches that would allow them to investigate further the causes for the regime changes. Since we have seen that regimes reoccur, we cannot rule out the fact that it is possible for the causes to be put in place again, attempting to force a regime transition. Although only mentioned briefly, we also explained how the GBNs that were learnt can be used on future data in order to monitor whether or not the decisions of the coaches are transitioning their players to favourable regimes. 
We have also seen that learning a single $\mathrm{BN}$ when regimes do occur results in a model that is worse at explaining the data, than are the individual $\mathrm{BNs}$ for each regime. It happens to be the case that we are analysing career data of baseball players in the current setting, however regardless of the context it is important to acknowledge that regime transitions may occur. To further this point, we shall now turn our attention to an application in a different domain, namely that of finance. We shall see how we can learn GBNs that model volatility regimes in financial markets, and how these correspond to the financial turmoil of the most recent financial crisis of 2007-08.

\subsection{Volatilty regimes in financial markets}

Our results on both sets of synthetic data and on the career data of baseball players are encouraging, indicating that if there are regimes in the underlying system, then our proposed learning algorithm is capable of identifying them. In this section we would like to further show the usefulness of modelling regimes, and will to this end investigate if we can identify volatility regimes in financial markets. The aim is to identifying how the relationships among different financial markets' volatility changes over time. The benefit of identifying regimes in these relationships is twofold. Firstly, the fact that a system is in a particular regime may be of value in and of itself. While our proposed learning algorithm does not attempt to label the regimes in any sense, in a real-world system it may be the case that some regimes can be seen as indicative of abnormal systemic behaviour. Secondly, if the goal is to use the BNs for inference purposes, then identifying which one to use at different times will lead to better results, than simply using the same $\mathrm{BN}$ across all regimes.

\subsubsection{Methodology}

There exists several approaches to measuring the volatility of a financial asset or market. For option traders, the implied volatility is of importance when calculating prices using the Black-Scholes model, while for other investors historical volatility may be of interest. Both these approaches require their own parameterisations and optimisations, which for our purpose we would like to avoid. We will therefore use the approach proposed by Welles Wilder [52], known as the true range (TR). 

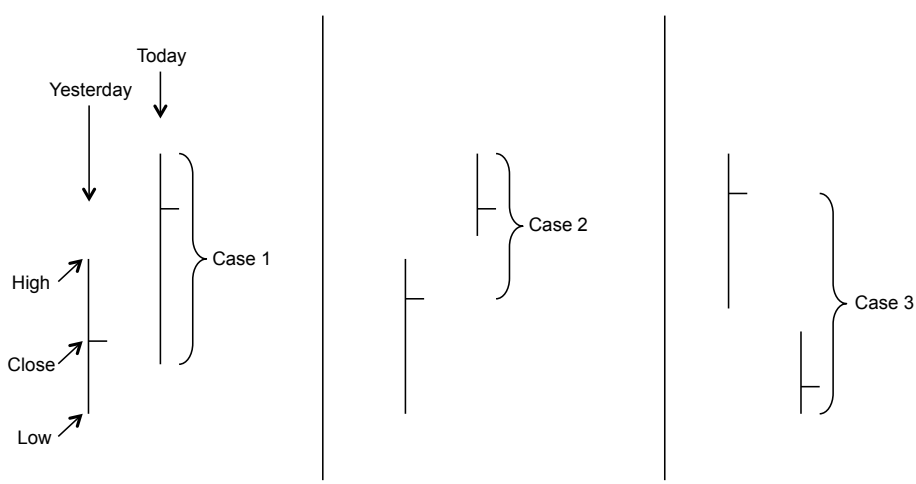

Figure 4.12: The greatest of the three cases shown is today's true range.

\section{Measuring volatility using true range}

Once the trading day comes to an end, and the market closes, prices of the assets traded on the market are quoted by the price of the asset when the market closed, i.e. closing prices. However, throughout the day an asset's price changes. The TR measures volatility defined by the range of the asset's price throughout the day. An asset for which its price has been almost constant throughout the day has low volatility, while an asset that has traded at a wide range of prices has high volatility. Since prices can jump between trading days, the TR utilises the greatest value of three: difference between today's high and low, difference between yesterday's close and today's high or the difference between yesterday's close and today's low. This is illustrated in Figure 4.12, where the vertical bars represent the price of an asset throughout a trading day, and the shorter horizontal bars represent the closing price.

\section{Data sets and procedure settings}

We devised two experiments, one that investigated the relationships among five major US based financial assets, and one that focused on assets in European stock markets. We will refer to these experiments as MFA and ESM. Each asset's TR is represented as a random variable in the BNs. Specifically, the assets considered in each of these experiments, along with their symbol, is listed in Table 4.10. For both experiments we used data ranging from 200605-08 to 2015-11-06.6 each variable discretised into four equal width bins.

During MH we used $k=8$ splits, thus we are not assuming any knowledge about how many splits there truly are in the data, only that there are a

\footnotetext{
${ }^{6}$ At the time of the data collection, these were the two dates that gave us the largest complete data set for all assets.
} 
Table 4.10: Data used in the MFA and ESM experiments.

\begin{tabular}{|l|l|}
\hline Symbol & Explanation \\
\hline \hline MFA experiment $\dagger$ \\
\hline SHY & $1-3$ year US treasury bond \\
\hline IEF & $7-10$ year US treasury bond \\
\hline IVV & Standard and Poor's 500 index (US stocks) \\
\hline IAU & Gold spot price \\
\hline IEO & Index of companies related to oil and gas \\
\hline \hline ESM experiment $\dagger$ \\
\hline EWQ & Large and mid-sized companies in France \\
\hline EWG & Large and mid-sized companies in Germany \\
\hline EWU & Large and mid-sized companies in United Kingdom \\
\hline IAU & Gold spot price \\
\hline IEO & Index of companies related to oil and gas \\
\hline \hline
\end{tabular}

${ }^{\dagger}$ BlackRock ${ }^{\circledR}$ electronically traded funds are used in the experiments. Data downloaded from Yahoo! Finance ${ }^{\mathrm{TM}}$.

maximum of eight. We ran 100000 iterations, throwing away the first 50 000 samples as burn-in. Again, we used the greedy thick-thinning algorithm introduced in Section 2.1.3 for learning the $\mathrm{BN}$ structures. We ran 250 iterations of Bayesian optimisation using the radial basis kernel with $c=2.0$, and the upper confidence bound criterion acquisition function using $\eta=5$. The objective function was the average accuracy over 1000 resamples.

\subsubsection{Results and discussion}

For each experiment (MFA and ESM) we report the learnt regimes and transition structure in the form of the full GBN, as well as a time series plot that shows the TR for each asset, overlaid with the identified nonzero $\delta$ s and the regime labels. To emphasise that the BNs contained within the GBN are better at describing the data in their respective regime, we also report the log marginal likelihood of each subset given the other BNs. Finally we report the log marginal likelihood of each subset of the data set given a BN that is learnt using the entire data set, i.e. learning a single $\mathrm{BN}$ disregarding any regimes.

\section{Results for MFA experiment}

For the MFA experiment, three nonzero $\delta$ s were identified, resulting in four subsets. However, the first and last subsets were found to belong to the same regime, resulting in the regime transition structure represented by the learnt GBN in Figure 4.13. The optimised trigger logic parameters were $\tau=26$ and $\theta=1.38$, comparable with the synthetic data set results in Table 4.3 . The TR for the assets in the MFA experiment are plotted in Figure 4.14, along with 
the nonzero $\delta$ s. In Figure 4.14, regime labels have been added below the data to show which regime each subset belongs to. In Table 4.11 the log marginal likelihood of each subset given each BN in the GBN is presented, along with the log marginal likelihood of each subset given a single BN learnt using all data.

\section{Results for ESM experiment}

Three nonzero $\delta$ s were also identified for the ESM experiment, resulting in four subsets. The first and fourth subsets were found to belong to the same regime, resulting in the regime transition structure represented by the learnt GBN in Figure 4.15 $(\tau=49$ and $\theta=1.49)$. The TRs along with nonzero $\delta$ s and regime labels are presented in Figure 4.16, and the log marginal likelihoods in Table 4.12. 
Table 4.11: Log marginal likelihood of data subsets given individual BNs (MFA).

\begin{tabular}{|l|l|l|l|l|}
\hline & Subset 1 & Subset 2 & Subset 3 & Subset 4 \\
\hline$R_{1}$ & $\mathbf{- 1 2 0 . 3 5 6}$ & -305.924 & -409.771 & $\mathbf{- 7 9 9 . 6 3 9}$ \\
\hline$R_{2}$ & -123.979 & $\mathbf{- 2 9 3 . 4 4 1}$ & -413.630 & -810.785 \\
\hline$R_{3}$ & -125.312 & -311.850 & $\mathbf{- 3 9 1 . 9 6 0}$ & -817.500 \\
\hline \hline Single BN & -121.027 & -299.296 & -398.726 & -807.713 \\
\hline
\end{tabular}

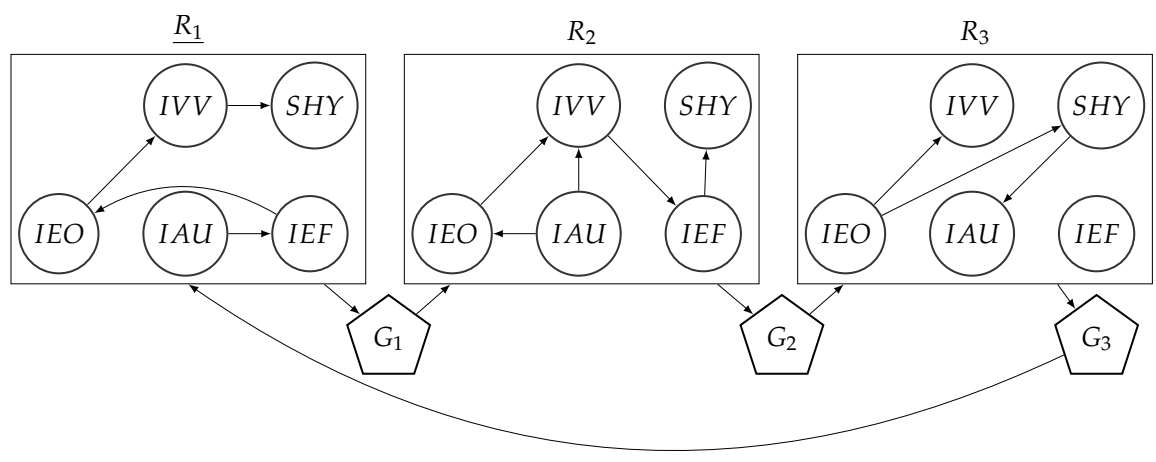

Figure 4.13: GBN learnt in the MFA experiment.

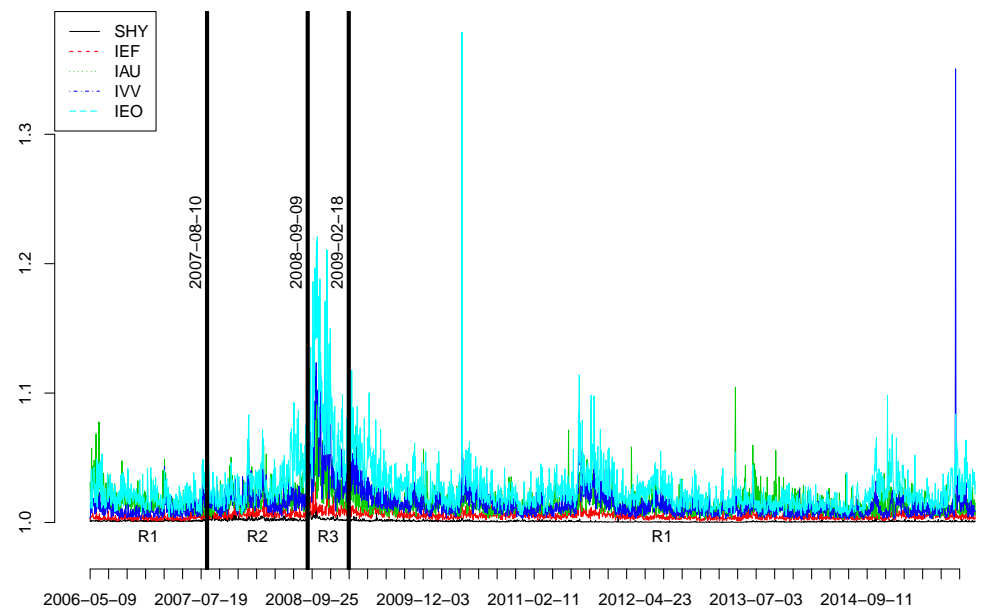

Figure 4.14: True range for assets in MFA with identified splits. 
Table 4.12: Log marginal likelihood of data subsets given individual BNs (ESM).

\begin{tabular}{|l|c|c|c|c|}
\hline & Subset 1 & Subset 2 & Subset 3 & Subset 4 \\
\hline$R_{1}$ & $\mathbf{- 3 3 0 . 8 8 4}$ & -584.422 & -614.648 & $\mathbf{- 1 8 3 . 4 5 8}$ \\
\hline$R_{2}$ & -337.876 & $\mathbf{- 5 5 9 . 1 0 4}$ & -586.675 & -186.094 \\
\hline$R_{3}$ & -343.965 & -576.253 & $\mathbf{- 5 6 8 . 1 5 3}$ & -183.771 \\
\hline \hline Single BN & -339.489 & -562.349 & -577.087 & -184.750 \\
\hline
\end{tabular}

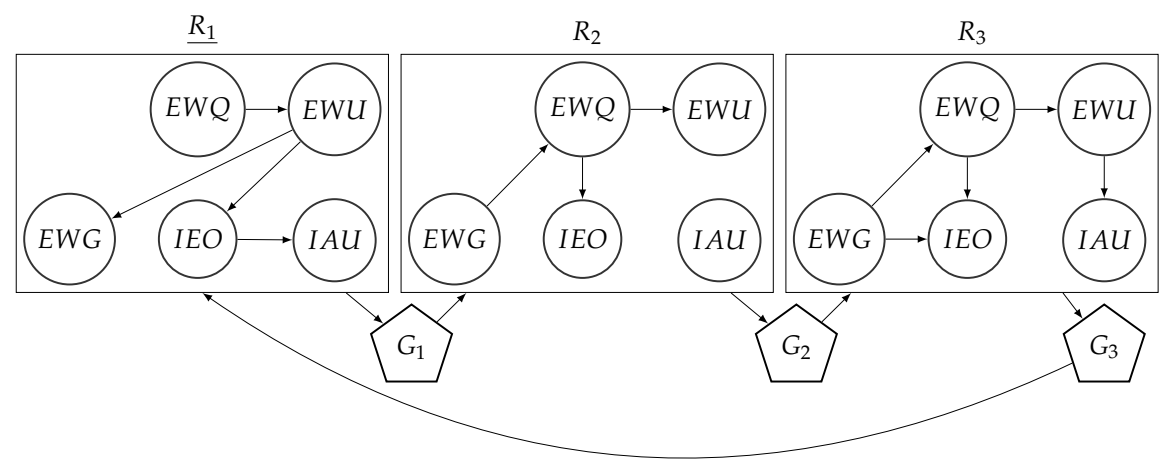

Figure 4.15: GBN learnt in the ESM experiment.

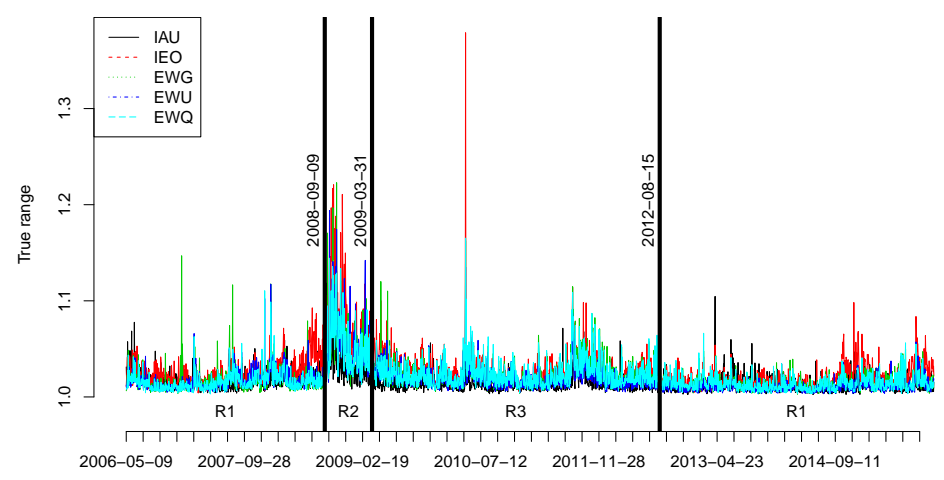

Figure 4.16: True range for assets in ESM with identified splits. 


\section{Discussion}

From the results of the experiments it is immediately clear that our learning algorithm was able to identify regimes in the volatility data. Moreover, and more importantly, the learning identified reoccurring regimes. While nobody is proclaiming that relationships among financial markets are stable, thus it is natural to find changing relationships, the possibility of identifying and modelling recurrences of these relationships is intriguing. Our procedure does not label the identified regimes, however we will here attempt to give a narrative to the regime transitions in our experiments. This is the best we can do, since we do not know the true regimes, and thus we cannot calculate the accuracy as with the simulated data.

Looking at the time series plot for the MFA experiment in Figure 4.14, we see that there are three regimes, where $R_{1}$ is in effect before 2007-08-10 and after 2009-02-18. The regime change from $R_{1}$ to $R_{2}$ happens during a time of increased fear in the financial markets of 2007. Leading up to August of 2007, several events began to indicate that mortgage backed securities were not as safe as previously thought. Funds investing in these types of securities were sharply losing value, and on 2007-07-31 Bear Stearns liquidated two of its hedge funds that were heavily invested in mortgage backed securities. The true indication that these securities were in fact problematic came in 200708-09, when the French bank BNP Paribas froze three of their funds due to the inability to value the complex assets inside them, i.e. packages of subprime mortgages. After the crisis, the Financial Crisis Inquiry Commission stated in their final report that: "In retrospect, many investors regarded the suspension of the French funds as the beginning of the 2007 liquidity crisis" [96]. The BNP Paribas event was really a liquidity crisis, i.e. freezing the fund means no money in or out, thus there is no liquidity in the asset. The Office of Financial Research found that when modelling the state of global market liquidity, 2007-08-09 stands out as an extreme liquidity state, possibly indicative of the coming crisis [97]. Timothy Geithner, then president of the Federal Reserve Bank of New York, later to be treasury secretary during the first term of the Obama administration, pointed out that for a fund to decline $20 \%$ is one thing, but to say that you cannot estimate the value of the fund, and then freeze the fund, is much worse [98]. He also reflects that "I knew what financial crises felt like, and they felt like this". We identified the regime change at approximately the same time as the BNP Paribas event, indicating that the financial markets were starting to change.

The new regime $\left(R_{2}\right)$ stays up until 2008-09-09, when a third regime $R_{3}$ is identified. Only two days earlier, on 2008-09-07, the US government decided 
to bail out Fannie Mae and Freddie Mac, two firms that guaranteed a large part of the existing sub-prime mortgages, and on 2008-09-15 the American bank Lehman Brothers filed for bankruptcy, leaving the rest of the financial world in panic. The Lehman Brothers bankruptcy was the most complex and far reaching bankruptcy in US history. Stock markets plummeted the day after the filing, wiping out $\$ 700$ billion in value from retirement plans, government pension plans and other investment portfolios [96]. The regime change we identified is again timely with these events. There were concerns about what the bailout would entail, and rumours of the failure of Lehman Brothers had been going around for some time, so it is natural that we see a change before the actual failure of Lehman Brothers, as markets begin to price and adjust for possible future events.

The third regime stays in effect during the height of the financial crisis, and then transitions back to the first regime $\left(R_{1}\right)$ on 2009-02-18, a date that is not easily pinpointed to a specific event. However, throughout late 2008 and early 2009 a large number of easing initiatives by the US Federal Reserve were carried out, as well as legislative actions to bolster the economy. The financial panic had abated, and later the National Bureau of Economic Research would officially determine that a trough in the business cycle was hit in June 2009, thus officially ending the recession. The regime change in volatility came earlier, however this is natural given that business cycle decisions are based on lagging indicators, such as gross domestic product and unemployment rates. The final regime, interestingly, is identified as being equal to the regime in place before the financial crisis, suggesting that this is a state of normality with respect to the probabilistic relationships amongst the financial markets.

From the GBN in Figure 4.13 it is clear that the independence model changes between regimes. For instance, we can see that the relationship between $I E O$ and $I A U$ is mediated through IEF in $R_{1}$, while the relationship is direct in $R_{2}$, and the direct relationship between $I V V$ and $S H Y$ in $R_{1}$ is lost in favour of a mediated relationship through IEF. During the worst part of the financial crisis $\left(R_{3}\right)$ the independence model changes dramatically. This is not only a local change between two variables, it is a shock to the entire system. Our approach shows its strengths here, as it does not make any assumption that the change is small between regimes. The regime lasts for approximately five months, equivalent to 111 data points, thus assuming small changes between regimes would either result in several successive regime changes or missing some of the changes completely.

We will here not attempt to explain what the differences between independence models represent, nor what the consequences may be from a financial 
and economical perspective, but rather be satisfied by the comparison of log marginal likelihoods in Table 4.11. The table shows that the BN designated to a regime scores better on the data belonging to that particular regime than any of the other BNs in the GBN. Also, on the last row of the table we present the log marginal likelihood for each subset of the data given a single BN learnt using all the data (i.e. using a traditional approach to learning a single $\mathrm{BN}$ ). As is evident, using the correct $\mathrm{BN}$ in accordance with the current regime yields a better representation of the data.

In the ESM experiments a similar narrative can be expressed, however regime shifts are not aligned exactly with MFA. This is quite likely due to the European scope of the variables: in MFA only financial markets closely related to the US economy were modelled. We again see a regime shift in the volatility relationships on 2008-09-09 (the same day as MFA), from $R_{1}$ to $R_{2}$. This is the Fannie Mae, Freddie Mac and Lehman Brothers events, which sets the markets in a new regime that holds until 2009-03-31. It seems that the ESM variables were not affected in the same way as the MFA variables with respect to the BNP Paribas event in August 2007. It is noteworthy that the problematic mortgage backed securities were US based, i.e. European markets may not have been affected as heavily as US markets by the BNP Paribas event, and it took a global event such as the Lehman Brothers filing for the crisis to become truly contagious. The regime $R_{2}$ transitions into $R_{3}$ in March 2009 (again, officially the US recession ended in June 2009). The ESM variables are in $R_{3}$, up until 2012-08-15, when they go back to regime $R_{1}$. The regime $\left(R_{3}\right)$ that is in place from late March 2009 until August 2012 is indicative of the remaining issues that lingered in Europe after the financial crises abated in the US. There was an ongoing sovereign debt crisis in smaller European countries, such as Greece, Ireland and Portugal, and another stock market fall ensued in August 2011 after Standard \& Poor's downgraded the US government's credit rating from AAA to AA+, which fuelled the concern that the sovereign debt crisis would spread into previously strong European economies, including Italy, Spain and France. On 2012-09-06 the European Central Bank announced free unlimited support for all eurozone countries that partook in precautionary programmes, timely with the return of the ESM variables back to the normal regime $R_{1}$.

As was the case with the MFA experiment, it is clear from the GBN in Figure 4.15 that the independence model changes between regimes. For instance, effects of EWU on IAU are mediated by IEO in $R_{1}$, and in $R_{2} I A U$ is disconnected from the rest of the variables. In $R_{3}$ the relationship between $E W U$ and $I A U$ is direct. In Table 4.12 the log marginal likelihoods are presented for each BN and each subset of the data, as well as the log marginal 
likelihoods of a single BN learnt using all the data. Again, we see that using the corresponding $\mathrm{BN}$ for each regime gives a better representation of the data.

\subsection{Conclusions and summary}

Change is inevitable. Yet we often seek to parameterise models that assume that relationships among measured phenomena are static. In the case of identically distributed observations in cross-sectional studies this type of assumption can be considered permissible. However, when we measure phenomena over time, and our reasoning requires knowledge about their interrelationships, then assuming that these stay static over time may not be as defensible. Of course, perceived relational differences from one observation to another may simply be due to the randomness of the system under study, thus it is not necessarily so that the joint probability distribution has changed. But when relations do change, thereby altering the joint probability distribution, then it is important that the model's structure reflects this.

In the introduction to this chapter we stated our aim to identify the process structure itself, rather than having it fixed a priori as in Chapter 3. We said that the central theme was to use GBNs as generative models for a set of random variables, and attempt to capture changes among the relationships over time. Throughout this chapter we have introduced a learning algorithm for this purpose and have through both synthetic and real-world experiments shown how this approach leads to learnt GBNs that achieve good results for both representing the data generating process, and detecting current regimes.

There are two things that stand out in our synthetic experiments. First, our learning algorithm was able to identify the correct transition structure in all experiments, something that we find very encouraging. We attribute this to the fact that GBNs are not mixture models, as can be deduced from the experiments in Appendix $B$ and $C$ the mixture models sometimes struggle to separate the individual regimes. Secondly, the GBNs performed well at identifying the current regime, i.e. the average accuracy of the learnt GBNs were high. This is important since we found that the BNs for the individual regimes represented their respective data better than the other BNs, thus it is important that the correct $\mathrm{BN}$ be active in the GBN.

Through our real-world experiments we wish to communicate how the learning algorithm and resulting GBNs can be used in different contexts. In the baseball setting we are promoting the GBN model as a tool for managers and coaches to identify regime transitions and in extension help them to put 
into place the conditions to promote (or avoid) extraordinary (or subpar) performance. In the financial domain we see the model more as a detection and confirmation tool of changes in the underlying markets. We have seen throughout the experiments in this chapter that learning a single model when regimes do occur results in a model that is worse at explaining the data than are the individual models for each regime.

The last sentence of the previous paragraph initiated the line of research that we shall account for next. Leaving GBNs aside for a moment, we asked if we can simply ensure that we have the most appropriate model at all times, while taking into consideration that we wish to have distinct models (i.e. not mixtures) and that there are regimes in the data that may reoccur. We also wanted to attempt to have a procedure that did not require access to all data, but instead worked in a more online fashion (i.e. receiving one data point at a time and finding the most appropriate model). In the next chapter we shall describe our approach to this problem. 


\section{Chapter 5}

\section{Regime aware learning of Bayesian networks}

In this chapter we shall continue our consideration of regime aware learning, treating a variation of the problem expressed in Chapter 4 The difference here will be that we shall focus on making available the best model for each data point that we collect. We will not go so far as to learn a full GBN, but rather a sequence of $\mathrm{BNs}$ where the last $\mathrm{BN}$ in the sequence represents the current regime, and should be used for inference purposes.

For the explanation of the algorithm and the experiments in this chapter it is natural to think of an investigator who is observing some system and receiving data at some interval (not necessarily fixed). The investigator feeds each new data point that is collected into the proposed algorithm and receives back the most appropriate model. The proposed algorithm reuses the model from the previous iteration as it processes the next available data point. In this sense the approach we are proposing in this chapter has an online flavour to it (rather than the batch data approach discussed in Chapter 4).

We shall begin by explaining the algorithm that we are proposing in Section 5.1. followed by a set of experiments on synthetic data in Section 5.2. In Section 5.3 we shall return to our investigation of volatility regimes in financial markets, after which we shall summarise our findings in Section 5.4 .

\subsection{Regime aware learning algorithm}

The aim of the learning algorithm is to supply the model that best represents the current regime to the investigator, and to update this model each time there is new data available. We begin by accounting for the steps that the algorithm takes each time new data is available, and then explain the details of each step in the subsequent sections. 
Let $\mathcal{D}$ represent an ordered data set, and let a hypothesis $H$ be a division of $\mathcal{D}=\left\{d_{1}, \ldots, d_{n}\right\}$ into subsets. Let $\mathcal{L}$ represent an algorithm for learning the structure and parameters of a $\mathrm{BN}$, and let $\mathcal{L}\left(\left\{d_{l}\right\}\right)$ mean that $\mathcal{L}$ has been used to learn a $\mathrm{BN}$ using the data point $d_{l}$. A model $M$ is then created by using $\mathcal{L}$ to learn a $\mathrm{BN}$ for each subset defined by a hypothesis $H$. For instance, given five data points, the hypothesis $H\{2,4\}$ splits the data at position 2 and 4, resulting in a model $M$ with the three BNs $\mathcal{L}\left(\left\{d_{1}\right\}\right), \mathcal{L}\left(\left\{d_{2}, d_{3}\right\}\right)$ and $\mathcal{L}\left(\left\{d_{4}, d_{5}\right\}\right)$. Notation wise, we will always let a hypothesis with a specific subscript define a model with the same subscript, i.e. the hypothesis $H_{k}$ defines the model $M_{k}$.

Each iteration of the algorithm will result in one hypothesis that defines the current model, we denote the current hypothesis by $H_{C}$ and the current model by $M_{C}$. The learning algorithm consists of the following five steps, which are run each time a new data point $d_{i}$ is made available. Before the first iteration, set $H_{C}=\varnothing$ and $\mathcal{D}=\varnothing$.

1. Add $d_{i}$ to the end of $\mathcal{D}$.

2. Create a set of hypotheses $\mathbf{H}$ by calling $\operatorname{PROPOSE}\left(H_{C}, \mathcal{D}\right)$.

3. Find $H_{\max }$ such that $H_{\max }=\arg \max _{H_{j} \in \mathbf{H}} p\left(M_{j} \mid \mathcal{D}\right)$.

4. Set $H_{C}=H_{\max }$ if $p\left(M_{\max } \mid \mathcal{D}\right)>p\left(M_{C} \mid \mathcal{D}\right)$.

5. Return to the investigator $M_{C}=\operatorname{MERGE}\left(H_{C}, \mathcal{D}\right)$. The $\mathrm{BN}$ in $M_{C}$ which was learnt using the subset of $\mathcal{D}$ containing $d_{i}$ represents the current regime.

The algorithm creates a set of hypotheses by calling PROPOSE, and then finds the hypothesis $H_{\max }$ that defines the model $M_{\max }$ with the highest posterior probability $p\left(M_{\max } \mid \mathcal{D}\right)$. If $p\left(M_{\max } \mid \mathcal{D}\right)>p\left(M_{\mathcal{C}} \mid \mathcal{D}\right)$, i.e. if the posterior odds is greater than one in favour of the hypothesised model, then $H_{C}$ is replaced by $H_{\max }$. The MERGE procedure then exhaustively combines the subsets defined by $H_{C}$, in order to identify reoccurring regimes, and the final model $M_{C}$ is returned to the investigator. In the following sections we will begin by explaining the procedure PROPOSE, followed by how the posterior $p(M \mid \mathcal{D})$ is calculated, and finally the MERGE procedure. We note here that the algorithm does not assume that any splits exist in the data, as the initial $H_{C}$ is the empty set, and splits are only added to $H_{C}$ if the posterior odds are in favour of such an addition. 


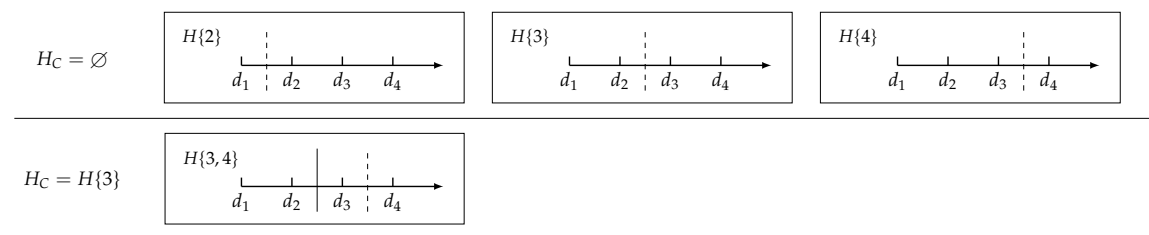

Figure 5.1: Effect of constraint on hypothesis generation.

\subsubsection{Proposing hypotheses}

In step 2 of the algorithm, PROPOSE creates a set of hypotheses $\mathbf{H}$, which becomes a search space in step 3. If every possible hypothesis was to be proposed, then the search space would expand beyond computational feasibility as $\mathcal{D}$ grows. We therefore introduce the following constraint, and give two examples that highlight where this constraint plays a role in the procedure:

Each hypothesis in $\mathbf{H}$ has the splits in $H_{C}$, plus a new one that is after the last split in $H_{C}$.

Figure 5.1 depicts two different outcomes of using PROPOSE when a data set containing four points is available. The top example in the figure depicts the case where $H_{C}$ contains no splits. This situation has been reached by rejection of all hypotheses when $d_{1}, d_{2}$ and $d_{3}$ were collected. In this case there are three possible hypotheses, illustrated by dashed lines in the figure. Note that due to the constraint we cannot add two splits, e.g. the hypothesis $H\{2,3\}$ is not generated since it has two more splits than $H_{C}$. In the bottom example in the figure, $H_{C}$ contains one split at data point three (illustrated by a solid line). The only hypothesis generated in this case is $H\{3,4\}$, since due to the constraint, we cannot propose splits before the existing split at data point three.

\subsubsection{Posterior of a model}

As we have seen from the discussion regarding the PROPOSE procedure, the number of hypotheses that are proposed is $n-d_{\text {last }}$, where $d_{\text {last }}$ is the last split in $H_{C}$ (or 1 if $H_{C}=\varnothing$ ). Therefore we must be able to choose one of these, and then compare this hypothesis with the current hypothesis in order to decide if it should be replaced. We do this by finding the hypothesis with the highest posterior $p(M \mid \mathcal{D}) \propto p(\mathcal{D} \mid M) p(M)$.

Let $H$ be a hypothesis with $k>0$ splits $\delta_{1}, \ldots, \delta_{k}$, then the model $M$ that $H$ defines consists of $k+1$ BNs. Also let $\mathcal{D}$ contain $n$ data points, and let $\mathcal{D}_{l}^{j}$ represent the subset $\left\{d_{l}, \ldots, d_{j}\right\}(l \leqslant j)$. We calculate the marginal likelihood 
of the data $\mathcal{D}$ given the model $M$ by the product of the marginal likelihoods of its $k+1$ BNs. When $k>1$ we therefore have:

$$
\begin{aligned}
p(\mathcal{D} \mid M)= & p\left(\mathcal{D}_{1}^{\delta_{1}-1} \mid \mathcal{L}\left(\mathcal{D}_{1}^{\delta_{1}-1}\right)\right) p\left(\mathcal{D}_{\delta_{k}}^{n} \mid \mathcal{L}\left(\mathcal{D}_{\delta_{k}}^{n}\right)\right) \times \\
& \prod_{i=1}^{k-1} p\left(\mathcal{D}_{\delta_{i}}^{\delta_{i+1}-1} \mid \mathcal{L}\left(\mathcal{D}_{\delta_{i}}^{\delta_{i+1}-1}\right)\right)
\end{aligned}
$$

When $k=1$ only the first two factors of Equation 5.1 apply, and when $k=0$ we have $p(\mathcal{D} \mid M)=p(\mathcal{D} \mid \mathcal{L}(\mathcal{D}))$. Notice that the first $k-1$ BNs are equal among all hypotheses (since they share the splits in $H_{C}$ ), thus the marginal likelihoods for these BNs need only to be calculated once, and can be reused in all following iterations.

The PROPOSE procedure will generate hypotheses where some or all of the subsets contain very few data points. There is therefore a risk that single values that are considered extreme given the current model will suggest that the current model be replaced. To avoid situations where many single point regimes are identified, we define a prior $p(M)$ over the models induced by the hypotheses, such that small regimes are less probable. We first conclude that all hypotheses share the splits from $H_{C}$, thus the prior over this part of the model is equal among hypotheses. The hypotheses do however differ with respect to the two new subsets that are created on either side of the added split, one to the left and one to the right. If a hypothesis was to replace $H_{C}$, then the subset to the left of the added split would be locked in as a regime, however the right subset will continue to increase in size as new data points become available. We therefore define $p(M)$ such that the probability of a specific subset size on the left side is smaller than the equivalent on the right side.

Let $G_{c d f}$ represent the cumulative distribution of a geometric distribution, and let $G_{c d f}(m, q)$ represent the probability of a regime with $m$ data points, when the geometric distribution is parameterised with $0<q \leqslant 1$. We then define $p(M)$ by Equation 5.2, where $p\left(M_{C}\right)$ is equal among all hypotheses and therefore set to unity, and $G_{c d f}$ is taken to the $n$ :th power to scale against the unnormalised $p(\mathcal{D} \mid M)$.

$$
p(M)=p\left(M_{C}\right) G_{c d f}\left(\delta_{k}-\delta_{k-1}, q_{(l e f t)}\right)^{n} G_{c d f}\left(n-\delta_{k}+1, q_{(\text {right })}\right)^{n}
$$

We offer a stylistic view of $p(M)$ in Figure 5.2. In the figure, $H_{C}=$ $H\{4,8\}$, and the proposed hypothesis is $H\{4,8,13\}$. The black dot over $d_{12}$ 


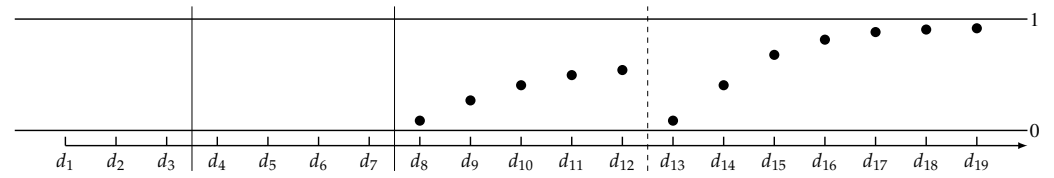

Figure 5.2: Stylistic view of the probabilities of the left and right subset sizes.

indicates the probability of a regime with five data points $\left(\mathcal{D}_{8}^{12}\right)$ given a geometric distribution parameterised with $q_{(\text {left })}$, while the black dot over $d_{19}$ indicates the probability of a regime with seven data points $\left(\mathcal{D}_{13}^{19}\right)$ given a geometric distribution parameterised with $q_{(\text {right })}$. As we can see, the probabilities grow as the size of the subsets increase, and $q_{(l e f t)}<q_{(\text {right })}$ following the previous discussion.

\subsubsection{Merging subsets}

The final step of the algorithm is to return the model implied by $H_{C}$ to the investigator. However, before doing so we perform one more task to improve upon the contained BNs. As we discussed in Section 4.2.2, if we naïvely assume that each split identifies a new regime we would have a chain of regimes, i.e. if there were two splits in $H_{C}$ we would have $R_{1} \rightarrow R_{2} \rightarrow R_{3}$. We would then return this model to the investigator and tell them to use the $\mathrm{BN}$ representing $R_{3}$ as a model for the current regime. However, since it is possible that $R_{2}$ transitioned back to $R_{1}$, and not into a new regime $R_{3}$, we could potentially get a better estimate of the model for the current regime by merging the subsets (thereby giving us more data to estimate structure and parameters from). We therefore apply the exact same procedure as described in Section 4.2.2, trying each possible recursive merging of nonadjacent subsets, as defined by the splits in $H_{C}$, and score a new model based on these new subsets. The model for which the merging leads to the best marginal likelihood of the data is then returned to the investigator.

\subsection{Synthetic data experiments}

In order to investigate the benefit of using the proposed learning algorithm, we set up a series of experiments to create a baseline comparison against learning a single BN. We considered data sets containing between zero and three regimes shifts. Using the same notation as in Chapter 4, when considering data sets with $i$ shifts, coming from the $j$ :th possible transition structure, 


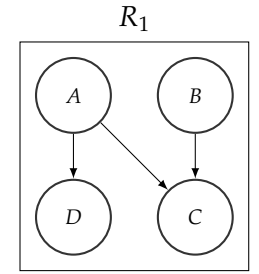

$A \sim N(1,0.5)$

$B \sim N(-1,0.5)$

$C \sim N(\sin (2 A+2 B), 0.1)$

$D \sim N\left(2 A^{2}, 0.1\right)$

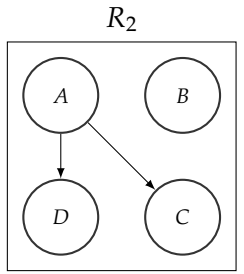

$A \sim N(1,0.5)$

$B \sim N(-1,0.5)$

$C \sim N(\sin (2 A), 0.1)$

$D \sim N\left(2 A^{2}, 0.1\right)$

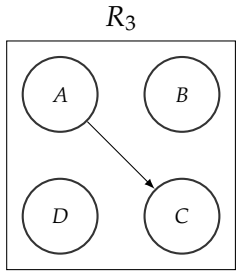

$A \sim N(1,0.5)$

$B \sim N(-1,0.5)$

$C \sim N(\sin (2 A), 0.1)$

$D \sim N(-2,0.5)$

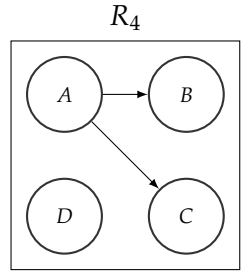

$A \sim N(1,0.5)$

$B \sim N\left(2 A^{2}, 0.1\right)$

$C \sim N(\sin (2 A), 0.1)$

$D \sim N(-2,0.5)$

Figure 5.3: BNs in set- $c$.

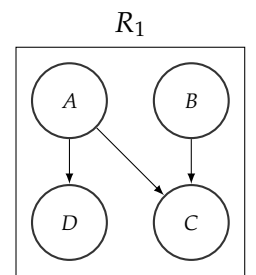

$A \sim N(1,0.5)$

$B \sim N(-1,0.5)$

$C \sim N(\sin (2 A+2 B), 0.1)$

$D \sim N\left(2 A^{2}, 0.1\right)$

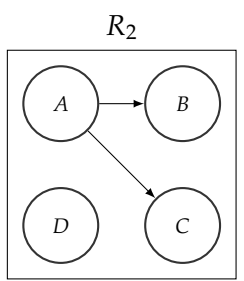

$A \sim N(1,0.5)$

$B \sim N\left(2 A^{2}, 0.5\right)$

$C \sim N(\sin (2 A), 0.1)$

$D \sim N(-2,0.5)$

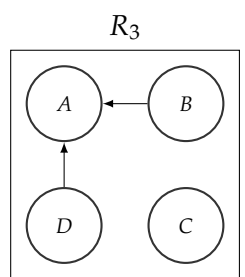

$A \sim N(\sin (2 B+2 D), 0.1)$

$B \sim N(-1,0.5)$

$C \sim N(2,0.5)$

$D \sim N(-2,0.5)$

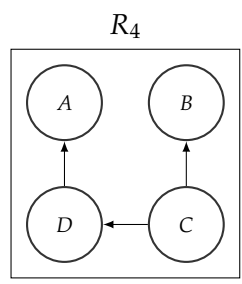

$A \sim N(\sin (2 D), 0.1)$

$B \sim N\left(C^{2}, 0.1\right)$

$C \sim N(2,0.5)$

$D \sim N\left(\sin \left(C^{2}\right), 0.1\right)$

Figure 5.4: BNs in set- $v$.

we will denote such a pair with $\mathcal{S}_{i, j}$. It is the transition structures in the left most column of Table 4.1 that were considered in these experiments.

We ran two separate experiments, which we will refer to as exp-c ( $c$ for calm) and exp- $v$ ( $v$ for volatile). For each experiment we set up a set of four BNs to represent the regimes. In the first set of BNs ( $\operatorname{Bet}-c$ ), we only added or removed one edge between regimes, thus there is still similarity between the BNs that make up the regimes. The BNs in set- $c$ are depicted in Figure 5.3, along with the individual variables' distributions. In the second set of BNs (set-v), we used the four BNs depicted in Figure 5.4, where there are more edge removals and additions between BNs, thus the similarity between regimes is drastically reduced.

\subsubsection{Bayesian network structure learning and priors}

For all experiments we used the greedy thick thinning algorithm described in Section 2.1.3 for learning the structures of the individual BNs. Since we were dealing with continuous data with non-linear relationships between variables, 
we did not use Dirichlet priors in the conditional distributions but rather used GP priors for variables with parents. When using GP priors in BNs they are sometimes referred to as GP networks [99]. Variables without parents were assigned a normal-inverse-gamma prior.

The prior $p(M)$ in Equation 5.2 was parameterised with $q_{(\text {left })}=0.05$ and $q_{\text {(right) }}=0.5$, thus it is less probable that small subsets will be accepted on the left side of a proposed split, as per the discussion in Section 5.1.2.

\subsubsection{Sampling}

For each experiment (exp-c and exp- $v$ ), we drew samples for each pair $\mathcal{S}_{i, j}$ by sampling the appropriate BNs. For instance, for $\mathcal{S}_{2,2}$ and exp-c we first drew a number of samples from $R_{1}$ in Figure 5.3, then a number of samples from $R_{2}$, and finally another number of samples from $R_{1}$. The number of samples drawn for each regime was picked at random from a uniform distribution between 50 and 70 .

\subsubsection{Methodology}

Given a sample, we processed each data point from the sample in turn and ran the proposed algorithm. Each time a new data point $d_{i}$ was made available for the algorithm, we calculated the log-likelihood of this data point given the current regime $\mathrm{BN}$ in $M_{C}$, and then incorporated $d_{i}$ into $\mathcal{D}$ and ran the rest of the algorithm. In parallel, we also calculated the log-likelihood of $d_{i}$ given a single BN learnt using all previous data, as well as the log-likelihood of $d_{i}$ using the proposed algorithm, but without the MERGE procedure. Thus for each sample we had three sets of log-likelihoods: $L_{s}, L_{n m}, L_{m}$ (single, no merge and merge), along with the mean values of these sets: $\bar{L}_{s}, \bar{L}_{n m}, \bar{L}_{m}$. The following analysis was done for each experiment and pair $\mathcal{S}_{i, j}$ :

- 50 samples were drawn from $\mathcal{S}_{i, j}$, resulting in three sets of means: $\left\{\bar{L}_{s}\right\}_{1}^{50},\left\{\bar{L}_{n m}\right\}_{1}^{50}$ and $\left\{\bar{L}_{m}\right\}_{1}^{50}$.

- Two null hypotheses were stated:

- $\mathcal{H}_{0}^{1}$ : The differences between pairs of means in $\left\{\bar{L}_{s}\right\}_{1}^{50}$ and $\left\{\bar{L}_{n m}\right\}_{1}^{50}$ follow a symmetric distribution centred at zero.

- $\mathcal{H}_{0}^{2}$ : The differences between pairs of means in $\left\{\bar{L}_{n m}\right\}_{1}^{50}$ and $\left\{\bar{L}_{m}\right\}_{1}^{50}$ follow a symmetric distribution centred at zero. 
- The Wilcoxon signed-rank test was used to test each null hypothesis. Two-tailed $p$-values below 0.01 were required to reject the null hypothesis.

\subsubsection{Results and discussion}

In Table 5.1 we present the results from the exp-c experiment. The first column gives the pair $\mathcal{S}_{i, j}$ under consideration, and the following three columns represent the median of the three sets $\left\{\bar{L}_{s}\right\}_{1}^{50},\left\{\bar{L}_{n m}\right\}_{1}^{50}$ and $\left\{\bar{L}_{m}\right\}_{1}^{50}$. The final two columns gives the $p$-values for the two Wilcoxon signed-rank tests. Table 5.2 gives the same values for the exp- $v$ experiment.

In both experiments, when there were no regime shifts $\left(\mathcal{S}_{0,1}\right)$, learning a single $\mathrm{BN}$ was significantly better than using the proposed learning algorithm. The reason is that it is possible that a series of closely located outliers may result in the algorithm deciding to introduce a split in the data set. However, the single BN never introduces this split, and it therefore has more data to estimate the parameters of the joint distribution of the single regime.

However, for all systems that exhibit regime changes, the proposed algorithm was significantly better than using a single BN. This was even true in the exp-c experiment, where the BNs of the different regimes retained more similarity. This result is very encouraging, as in a real-world setting we would not be able to test how much the BNs change between regimes, however as we have shown here, the proposed algorithm is sensitive to large as well as small changes.

The transition structures for $\mathcal{S}_{1,1}, \mathcal{S}_{2,1}$ and $\mathcal{S}_{3,1}$ are all chains of regimes. As expected, in both the exp-c and exp- $v$ experiments there was no significant difference between using the proposed algorithm with or without the MERGE procedure for these pairs. However, for all other pairs the difference was significant, with a lower median when including the MERGE procedure. When regimes reoccur, we can get better estimates of the BNs' parameters by using all data belonging to a specific regime, as is evident from the increased performance when using the MERGE procedure.

All in all, these baseline results confirm that the algorithm works as intended, adapting to regime shifts, and thereby supplying a $\mathrm{BN}$ which better represents the current regime than a $\mathrm{BN}$ learnt using all the available data.

\subsection{Revisiting volatility regimes in financial markets}

The baseline results from the experiments in Section 5.2 are promising, as they confirm that the proposed algorithm performs as expected. This 
Table 5.1: Results from the exp-c experiment.

\begin{tabular}{|l|l|l|l|l|l|}
\hline Pair & Median $\left\{\bar{L}_{s}\right\}_{1}^{50}$ & Median $\left\{\bar{L}_{n m}\right\}_{1}^{50}$ & Median $\left\{\bar{L}_{m}\right\}_{1}^{50}$ & $p$-value $\mathcal{H}_{0}^{1}$ & $p$-value $\mathcal{H}_{0}^{2}$ \\
\hline $\mathcal{S}_{0,1}$ & $\mathbf{- 3 . 3 5 0}$ & -3.487 & -3.487 & $<0.001^{*}$ & 1.000 \\
\hline $\mathcal{S}_{1,1}$ & -3.792 & $\mathbf{- 3 . 2 2 0}$ & $\mathbf{- 3 . 2 2 0}$ & $<0.001^{*}$ & 1.000 \\
\hline $\mathcal{S}_{2,1}$ & -4.063 & $\mathbf{- 3 . 1 5 5}$ & -3.160 & $<0.001^{*}$ & 0.955 \\
\hline $\mathcal{S}_{2,2}$ & -3.699 & -3.390 & $\mathbf{- 3 . 3 2 3}$ & $<0.001^{*}$ & $<0.001^{* *}$ \\
\hline $\mathcal{S}_{3,1}$ & -5.484 & $\mathbf{- 3 . 2 9 6}$ & -3.299 & $<0.001^{*}$ & 0.787 \\
\hline $\mathcal{S}_{3,2}$ & -3.987 & -3.482 & $\mathbf{- 3 . 2 9 8}$ & $<0.001^{*}$ & $<0.001^{* *}$ \\
\hline $\mathcal{S}_{3,3}$ & -4.020 & -3.212 & $\mathbf{- 3 . 0 6 0}$ & $<0.001^{*}$ & $<0.001^{* *}$ \\
\hline $\mathcal{S}_{3,4}$ & -4.095 & -3.477 & $\mathbf{- 3 . 1 9 6}$ & $<0.001^{*}$ & $<0.001^{* *}$ \\
\hline $\mathcal{S}_{3,5}$ & -3.717 & -3.214 & $\mathbf{- 3 . 0 8 3}$ & $<0.001^{*}$ & $<0.001^{* *}$ \\
\hline \multicolumn{5}{|r}{} & $*$ rejection of $\mathcal{H}_{0}^{1}, * *$ rejection of $\mathcal{H}_{0}^{2}$
\end{tabular}

Table 5.2: Results from the exp-v experiment.

\begin{tabular}{|l|l|l|l|l|l|}
\hline Pair & Median $\left\{\bar{L}_{s}\right\}_{1}^{50}$ & Median $\left\{\bar{L}_{n m}\right\}_{1}^{50}$ & Median $\left\{\bar{L}_{m}\right\}_{1}^{50}$ & $p$-value $\mathcal{H}_{0}^{1}$ & $p$-value $\mathcal{H}_{0}^{2}$ \\
\hline $\mathcal{S}_{0,1}$ & $\mathbf{- 3 . 3 5 0}$ & -3.487 & -3.487 & $<0.001^{*}$ & 1.000 \\
\hline $\mathcal{S}_{1,1}$ & -3.840 & $\mathbf{- 3 . 5 1 6}$ & -3.524 & $<0.001^{*}$ & 0.100 \\
\hline $\mathcal{S}_{2,1}$ & -5.100 & -3.939 & $\mathbf{- 3 . 9 3 3}$ & $<0.001^{*}$ & 0.027 \\
\hline $\mathcal{S}_{2,2}$ & -3.935 & -3.561 & $\mathbf{- 3 . 4 6 5}$ & $<0.001^{*}$ & $<0.001^{* *}$ \\
\hline $\mathcal{S}_{3,1}$ & -5.399 & $\mathbf{- 3 . 8 6 1}$ & -3.967 & $<0.001^{*}$ & 0.123 \\
\hline $\mathcal{S}_{3,2}$ & -4.790 & -3.691 & $\mathbf{- 3 . 6 0 9}$ & $<0.001^{*}$ & $<0.001^{* *}$ \\
\hline $\mathcal{S}_{3,3}$ & -5.084 & -3.943 & $\mathbf{- 3 . 8 9 5}$ & $<0.001^{*}$ & $<0.001^{* *}$ \\
\hline $\mathcal{S}_{3,4}$ & -5.051 & -4.129 & $\mathbf{- 3 . 9 3 9}$ & $<0.001^{*}$ & $<0.001^{* *}$ \\
\hline $\mathcal{S}_{3,5}$ & -3.838 & -3.407 & $\mathbf{- 3 . 1 5 5}$ & $<0.001^{*}$ & $<0.001^{* *}$ \\
\hline \multicolumn{5}{|l|}{ * rejection of $\mathcal{H}_{0}^{1}, * *$ rejection of $\mathcal{H}_{0}^{2}$} \\
\end{tabular}

prompted us to investigate the performance of the algorithm on real-world data. To this end we returned to the volatility regimes initially investigated in Section 4.6. We recall that the variables in the two data sets represented the daily TR of the following assets (please see Table 4.10 for more details of the assets):

- MFA: short-term US debt, long-term US debt, stocks of US companies, gold, and stocks of companies related to oil and gas.

- ESM: stocks of French companies, stocks of German companies, stocks of UK companies, gold, and stocks of companies related to oil and gas.

For these experiments we used data ranging between May of 2007 and May of 2009 (that is, enough data to encompass the most recent financial crisis), and we used GP priors in our BNs.

For each data set we ran the proposed learning algorithm and calculated the log-likelihood for each new data point (similar to the procedure 
described in Section 5.2.3. We then calculated the cumulative sum of these log-likelihoods. The series are plotted in Figure 5.5 for MFA and Figure 5.6 for ESM. In the figures, the solid line represents the proposed algorithm and the dotted line a single BN. Below the cumulative log-likelihoods we have plotted the data for each variable, along with the splits introduced by the algorithm (vertical solid lines). In this setting, data is made available on a daily basis, thus one iteration of the algorithm needs to be run each day, given the previous day's model. In our experiments, the average time to complete one iteration was approximately two minutes, thus giving the algorithm ample time to complete during the day. However, if working in a domain where data is more frequent than the time that the algorithm needs to complete, then a mini-batch mode framework or similar solution needs to be considered.

It is clear that up until September 2008 the performance of the two approaches are approximately equal, thus the identified splits up until this point may be considered spurious. Specifically, looking at Figure 5.5 we can see that several splits were identified in the data when learning a sequence of BNs. The reason for this is that learning a sequence of BNs has as its main goal to produce the best model for the most recent data point, rather than taking into consideration the entire data generating process (which was the case with the batch mode approach described in Chapter 4, which is also the reason to why we identify different splits in the current approach compared to the batch mode approach).

Past September 2008 the single BN's performance deteriorates compared to the proposed algorithm. As we narrated in Section 4.6.2, during the spring of 2007 it was becoming evident that some of the mortgage backed securities that were being traded, and used as collateral, were not as safe as previously thought. Bear Stearns liquidated two of its hedge funds in late July, and BNP Paribas froze three of their funds in August, as they were unable to price them. These issues escalated in 2008, and in September two major events occurred in the US financial system: first on 2008-09-07 the US government decided to bail out Fannie Mae and Freddie Mac, two firms that were guaranteeing a large part of the notorious sub-prime mortgage market, and then Lehman Brothers filed for bankruptcy on 2008-09-15. It was after these two events that the growing US financial crisis became a full blown global financial crisis. Figure 5.5 and Figure 5.6 suggest that there were regime shifts among the price volatilities measured during this period, and that the proposed algorithm is able to adapt to these shifts, since the cumulative log-likelihood does not dramatically change but rather seems to decrease at the same rate. Note that for the US centric MFA data set the shift in September 2008 is clearly captured, however as described in Section 4.6.2 the impact on EU markets was 


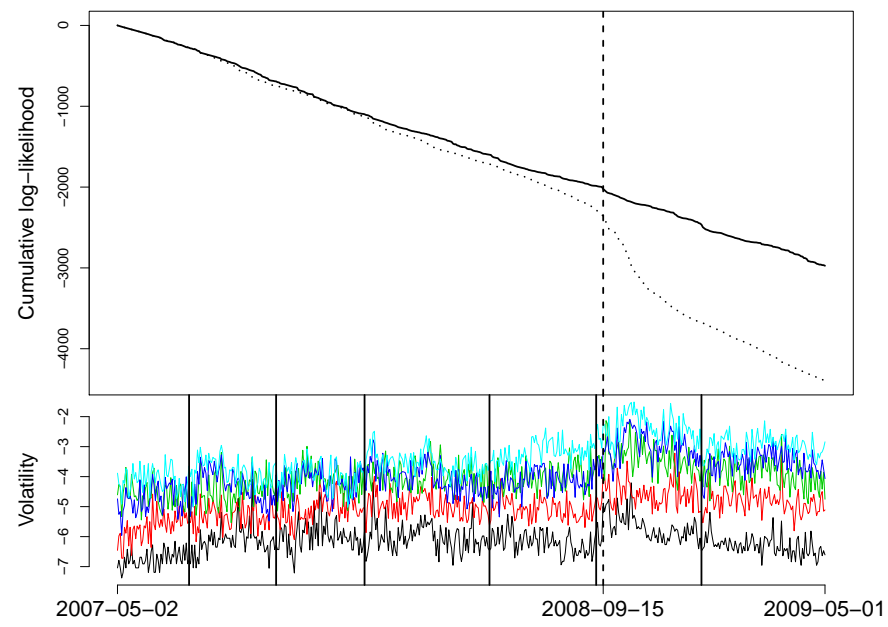

Figure 5.5: Cumulative log-likelihood and splits for the MFA data set.

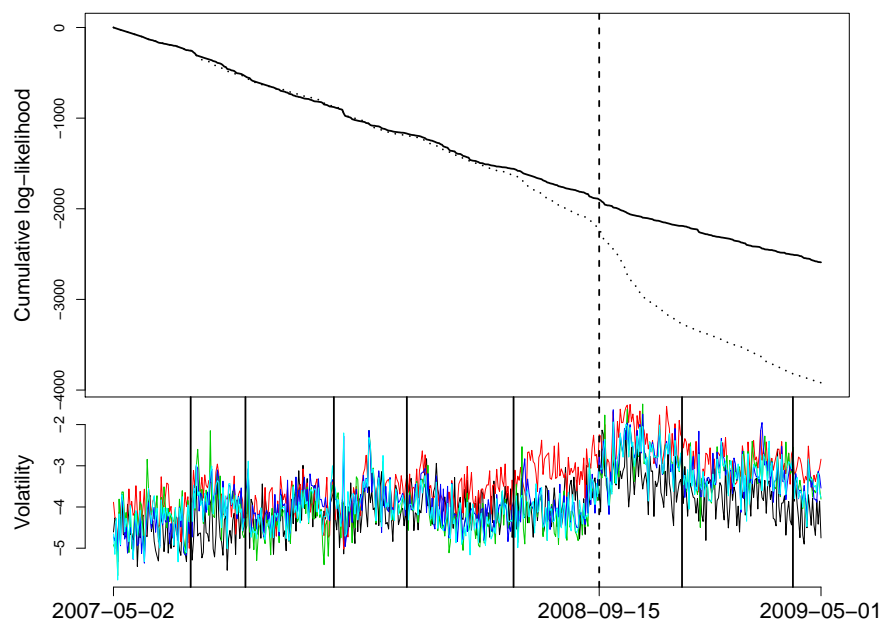

Figure 5.6: Cumulative log-likelihood and splits for the ESM data set.

not perfectly aligned with that of the US markets, thus a shift is not identified for the ESM data in September 2008.

\subsection{Summary}

The learning algorithm that we have explored in this chapter does not attempt to learn a full GBN, since a GBN aims to model the whole data generating 
process and we cannot do this following an online fashion. This also highlights why the two approaches can coexist, one to be used when the goal is inference right now, and the other when it is understanding the entire process that is of interest.

We shall continue our departure from learning and using GBNs, and will in the next chapter look at how we can extend traditional HMMs by using features inspired by GBNs. Briefly, we shall have a BN for each hidden state (in GBNs we have a BN for each phase or regime), and we shall allow for one of the variables in the BNs to influence the next hidden state (in GBNs we have variables that partake in the trigger logic of gates which dictate if we shall change state or not). Since the hidden states are unobservable, we end up with a model that mixes several BNs, from which one variable is partaking in the next choice of mixture weights. 


\section{Chapter 6}

\section{Modelling regimes using Bayesian network mixtures}

From the view of graphical models, the archetype approach for modelling states of the world is to use a HMM, where the regimes are modelled using hidden random variables, and we observe the random variables that we are modelling under different states of these hidden variables. When using standard HMMs, it is common to assume that the observable variables are independent of each other given the hidden variable, and not to model any potential dependencies among the observed variables directly.

In this chapter we shall propose an extension of the HMM, which we shall call GBN-HMM, where we extend the standard HMM with two features based on the fundamental ideas behind the GBN. First, we shall allow for different BNs over the observable variables under the different states of the hidden variables, to have a regime-dependent model over the observable variables. Second, we shall model a potential dependence between one of the observable variables and the next hidden state. The second extension stems from the use of posterior probabilities as part of the trigger logic of gates in GBNs.

In Section 6.1 we shall consider other existing extensions of the HMM found in the literature, that are related to the extensions that we shall propose. In Section 6.2 we will introduce and define the model that we are proposing, describing some of its underlying properties. Parameter estimation is not immediately straightforward since there are hidden variables in the proposed model, and we shall therefore explore how we can use expectation maximisation (EM) in Section 6.3 to estimate the parameters of our model. In Section 6.4 we wish to demonstrate the appropriateness of the GBN-HMM using synthetic data, and compare it with the HMM as well as two other HMM 
variants. We then turn our attention to using GBN-HMMs in a real-world situation, revisiting the domain of algorithmic trading in Section 6.5

\subsection{Related work}

HMMs have been applied and extended extensively throughout the literature, and we shall here not attempt an overview of all that has been explored. The interested reader may instead wish to consider the summary provided by [100]. Instead, we shall pay brief attention to a few existing variations that have a connection with the ideas that we are putting forward in this chapter.

A HMM where some control signal is given as input to the hidden state and the observable variables is described in [101], and an EM algorithm is offered to update the parameters of the observational and transition distributions conditional on a sequence of inputs. As a variation on this theme, [102] proposes that transitions between hidden states in a HMM may not only depend on the immediately previous state, but also on the immediately proceeding observation. This potential dependence between the observed variables at time $t$ and the hidden state at time $t+1$ is also present in the GBN-HMM that we are proposing. We shall use the model proposed in [102] as a comparison model in our experiments.

The auto-regressive HMM (AR-HMM), also know as the regime switching Markov model [64], incorporates potential dependence directly between an observable variable at time $t$ and its counterpart at $t+1$. While the ARHMM may be extended to higher orders, i.e. allowing for even longer dependence than only between $t$ and $t+1$, the dependence is between counterparts in each time slice. However, dynamic Bayesian multinets (DBMs) proposed in [103] allow not only for dependence across time slices among observational counterparts, but arbitrarily among the observed variables. Furthermore, DBMs allow these potential dependencies to change depending on the hidden states, thus allowing for a more complex dependence structure across time. The model that we are proposing does not include potential direct dependence among observable variables across time, but rather within each time slice. Allowing for different BNs for different states of the hidden variable in a HMM has also been developed simultaneously as (and independently from) our work, described as asymmetric HMMs in [104]. 


\subsection{Model definition}

The GBN-HMM that we are proposing consists of a set of discrete random variables $H_{1: T}=\left\{H_{1}, H_{2}, \ldots, H_{T}\right\}$ that represent the hidden state at each time $t \in[1, T]$. We call these the hidden state variables, and they each have the same number of possible states $N$. We use $h_{t}$ to denote a specific instantiation of the variable $H_{t}$, and use $h_{j: k}$ to denote a sequence of states from time $j$ to $k$. For each $t$, we will also model a set of discrete random variables $\mathbf{O}_{t}=\left\{O_{t}^{1}, O_{t}^{2}, \ldots, O_{t}^{M}\right\}$ for which we can observe their values. We will refer to these variables as the observable variables. We let $\mathbf{o}_{t}=\left\{o_{t}^{1}, o_{t}^{2}, \ldots, o_{t}^{M}\right\}$ be a particular instantiation of the observable variables at time $t$, and use $\mathbf{O}_{j: k}$ and $\mathbf{o}_{j: k}$ when considering all observable random variables and their respective values from time $j$ to $k$.

Since we wish to model the observable variables depending on the current state, we will have one $\mathrm{BN}$ for each state of the hidden state variable $H_{t}$, that is, there are $N$ BNs over the variables $\mathbf{O}_{t}$, and the value of $H_{t}$ selects one of these. One of the variables in $\mathbf{O}_{t}$ is of particular interest, as we will model a potential dependence between this variable and the state of $H_{t+1}$. We will refer to this variable as the $Z$ variable when we need to distinguish it from the other observable variables. Notation wise we let $Z_{t}$ represent the $Z$ variable at time $t$, and $z_{t}$ an instantiation of the $Z$ variable at time $t$.

Note that, although not made explicit, we have made use of certain independence assumptions among the variables $H_{1: T}$ and $\mathbf{O}_{1: T}$. First, we assume that $\mathbf{O}_{t}$ are conditionally independent of all previous random variables $\mathbf{O}_{1: t-1}$ and $H_{1: t-1}$, given the current hidden state variable $H_{t}$ (thus knowing the current state renders the past irrelevant). Second, the current hidden state variable $H_{t}$ is conditionally independent of $\mathbf{O}_{1: t-1} \backslash Z_{t-1}$ and $H_{1: t-2}$ given $H_{t-1}$ and $Z_{t-1}$ (thus knowing the value of the previous state and $Z$ variable renders the rest of the past irrelevant). We can represent these assumptions using a graph, an example of which is depicted in Figure 6.1. In the figure we can see that it is $O_{t}^{3}$ that is the $Z$ variable, as we are modelling a potential dependence between it and the next hidden state.

The final assumption that we will make is that of stationarity of the model. That is, the distributions and independencies that govern the model are independent of $t$. This implies that the probability of moving from one hidden state to another is the same regardless of $t$, and that the BNs selected by $H_{i}$ are the same as for $H_{j}$ for all $i, j \in[1, T]$. Furthermore, the $Z$ variable is always the same observable variable, regardless of $t$ or the state of $H_{t}$. 


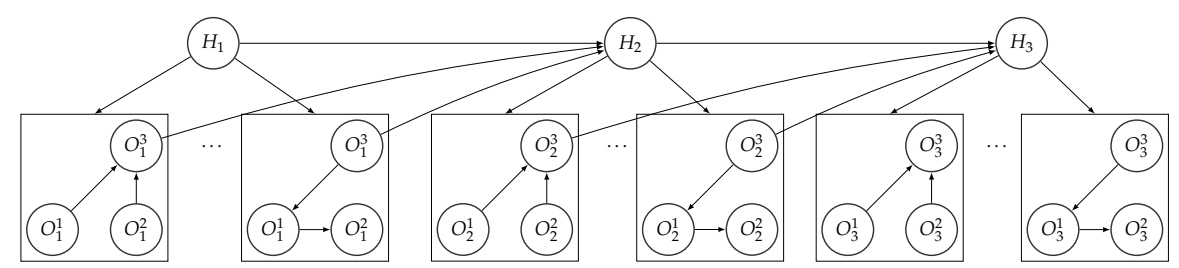

Figure 6.1: Graph representation of the GBN-HMM with three time steps.

\subsubsection{Factorisation}

As is the case with BNs, using the independence assumptions implied by the model, and the chain rule of probability, we can factorise the joint distribution over $H_{1: T}$ and $\mathbf{O}_{1: T}$ into marginal and conditional distributions that together require fewer parameters than the full joint. To illustrate this factorisation in a succinct manner, we shall factorise the GBN-HMM given in Figure 6.1. We assume that the hidden state variables have two states, i.e. $N=2$, however expanding this example to any number of observable variables, hidden states and time steps is straightforward. We begin the example by observing that we can isolate the variables $\mathrm{O}_{3}^{1}, \mathrm{O}_{3}^{2}$ and $\mathrm{O}_{3}^{3}$ by conditioning on $\mathrm{H}_{3}$ alone, which follows from Equation 6.1 .

$$
\begin{aligned}
& p\left(O_{1}^{1}, O_{1}^{2}, O_{1}^{3}, \ldots, O_{3}^{1}, O_{3}^{2}, O_{3}^{3}, H_{1}, H_{2}, H_{3}\right)= \\
& p\left(O_{3}^{1}, O_{3}^{2}, O_{3}^{3} \mid \varnothing_{1}^{\chi}, \varnothing_{1}^{2}, \varnothing_{1}^{\gamma}, \ldots, H_{1}, H_{2}, H_{3}\right) \times \\
& p\left(O_{1}^{1}, O_{1}^{2}, O_{1}^{3}, \ldots, H_{1}, H_{2}, H_{3}\right)= \\
& p\left(O_{3}^{1}, O_{3}^{2}, O_{3}^{3} \mid H_{3}\right) p\left(O_{1}^{1}, O_{1}^{2}, O_{1}^{3}, \ldots, H_{1}, H_{2}, H_{3}\right)
\end{aligned}
$$

Since the hidden variables in a GBN-HMM select among several BNs over the observable variables, the two states of $\mathrm{H}_{3}$ select between two joint distribution specifications over $O_{3}^{1}, O_{3}^{2}$ and $O_{3}^{3}$. If we let $\Pi_{j}\left(O_{3}^{1}\right)$ represent the parents of the variable $\mathrm{O}_{3}^{1}$ with respect to the DAG of the BN selected by $\mathrm{H}_{3}=j$, then we can continue the factorisation according to Equation 6.2 .

$$
\begin{aligned}
& p\left(O_{3}^{1}, O_{3}^{2}, O_{3}^{3} \mid H_{3}\right) p\left(O_{1}^{1}, O_{1}^{2}, O_{1}^{3}, \ldots, H_{1}, H_{2}, H_{3}\right)= \\
& p\left(O_{3}^{1}, O_{3}^{2}, O_{3}^{3}\right)^{\delta\left(H_{3}=1\right)} p\left(O_{3}^{1}, O_{3}^{2}, O_{3}^{3}\right)^{\delta\left(H_{3}=2\right) \times} \\
& p\left(O_{1}^{1}, O_{1}^{2}, O_{1}^{3}, \ldots, H_{1}, H_{2}, H_{3}\right)= \\
& \prod_{j=1}^{2} \prod_{i=1}^{3} p\left(O_{3}^{i} \mid \Pi_{j}\left(O_{3}^{i}\right)\right)^{\delta\left(H_{3}=j\right)} p\left(O_{1}^{1}, O_{1}^{2}, O_{1}^{3}, \ldots, H_{1}, H_{2}, H_{3}\right)
\end{aligned}
$$


In Equation 6.2 we let $\delta\left(H_{3}=j\right)$ represent the Kronecker delta, i.e. when $\mathrm{H}_{3}$ takes the value $j$ it equates to unity, otherwise zero.

The next step of the factorisation is to break out $\mathrm{H}_{3}$ from the remaining variables, which follows from Equation 6.3

$$
\begin{aligned}
& \prod_{j=1}^{2} \prod_{i=1}^{3} p\left(O_{3}^{i} \mid \Pi_{j}\left(O_{3}^{i}\right)\right)^{\delta\left(H_{3}=j\right)} \times \\
& p\left(H_{3} \mid \varnothing_{1}^{\chi}, \varnothing_{1}^{\chi}, \varnothing_{1}^{\gamma}, \varnothing_{2}^{\chi}, \varnothing_{2}^{\chi}, O_{2}^{3}, H_{1}, H_{2}\right) \times \\
& p\left(O_{1}^{1}, O_{1}^{2}, O_{1}^{3}, O_{2}^{1}, O_{2}^{2}, O_{2}^{3}, H_{1}, H_{2}\right)= \\
& \prod_{j=1}^{2} \prod_{i=1}^{3} p\left(O_{3}^{i} \mid \Pi_{j}\left(O_{3}^{i}\right)\right)^{\delta\left(H_{3}=j\right)} p\left(H_{3} \mid H_{2}, O_{2}^{3}\right) \times \\
& p\left(O_{1}^{1}, O_{1}^{2}, O_{1}^{3}, O_{2}^{1}, O_{2}^{2}, O_{2}^{3}, H_{1}, H_{2}\right)
\end{aligned}
$$

It should then be clear that we can continue the same operations for the remainder of the variables, ending the factorisation with a marginal distribution over $H_{1}$. The GBN-HMM factorisation for $T$ time steps, with $N$ hidden states and $M$ observable variables, is given in Equation 6.4 .

$$
p\left(H_{1}\right) \prod_{t=2}^{T} p\left(H_{t} \mid H_{t-1}, Z_{t-1}\right) \prod_{t=1}^{T} \prod_{j=1}^{N} \prod_{i=1}^{M} p\left(O_{t}^{i} \mid \Pi_{j}\left(O_{t}^{i}\right)\right)^{\delta\left(H_{t}=j\right)}
$$

\subsubsection{Likelihood}

Considering a specific sequence of observations $\mathbf{o}_{1: T}$ and hidden states $h_{1: T}$, we can use the factorisation to compute the likelihood of this data under a set of parameters $\Theta$. We let $\pi_{i}$ represent the probability $p\left(H_{1}=i \mid \Theta\right)$, $a_{i j k}$ the probability $p\left(H_{t}=j \mid H_{t-1}=i, Z_{t-1}=k, \Theta\right)$, and $b_{j}^{i}\left(\mathbf{o}_{t}\right)$ represent the probability $p\left(O_{t}^{i}=o_{t}^{i} \mid \Pi_{j}\left(O_{t}^{i}\right)=\mathbf{o}_{t}^{\Pi_{j}\left(O_{t}^{i}\right)}, \Theta\right)^{\delta\left(H_{t}=j\right)}$, where we let $\mathbf{o}_{t}^{\Pi_{j}\left(O_{t}^{i}\right)}$ represent the values that the parent set takes in $\mathbf{o}_{t}$. Then the likelihood $p\left(\mathbf{o}_{1: T}, h_{1: T} \mid \Theta\right)$ can be expressed by Equation 6.5 .

$$
p\left(\mathbf{o}_{1: T}, h_{1: T} \mid \Theta\right)=\pi_{h_{1}} \prod_{t=2}^{T} a_{h_{t-1}, h_{t}, z_{t-1}} \prod_{t=1}^{T} \prod_{i=1}^{M} b_{h_{t}}^{i}\left(\mathbf{o}_{t}\right)
$$

If we could observe both $\mathbf{o}_{1: T}$ and $h_{1: T}$ then estimating the parameters $\Theta$ that maximised the likelihood would be straightforward. However, since 
$H_{1: T}$ are hidden variables we cannot observe their values, and must therefore apply a more involved technique for estimating $\Theta$.

\subsection{Parameter estimation}

The canonical way of solving the parameter estimation problem in regular HMMs (and in their extensions) is to employ EM. We shall also adopt this approach, and in this section describe the computations necessary for iteratively updating the parameters $\Theta$ for the GBN-HMM that we are currently proposing.

As before, let $\mathbf{0}_{1: T}$ represent a sequence of observations over the variables $\mathbf{O}_{1: T}$ and let $h_{1: T}=\left\{h_{1}, h_{2}, \ldots, h_{T}\right\}$ represent a sequence of states. Let $\mathcal{H}$ represent the set of all state sequences $h_{1: T}$. The current parameters for our model are denoted $\Theta^{\prime}$, and we seek parameters $\Theta$ such that $p\left(\mathbf{o}_{1: T} \mid \Theta\right) \geqslant$ $p\left(\mathbf{o}_{1: T} \mid \Theta^{\prime}\right)$. It can be shown [105] that this task can be converted into a maximisation problem of:

$$
Q\left(\Theta, \Theta^{\prime}\right)=\sum_{h_{1: T} \in \mathcal{H}} p\left(\mathbf{o}_{1: T}, h_{1: T} \mid \Theta^{\prime}\right) \log p\left(\mathbf{o}_{1: T}, h_{1: T} \mid \Theta\right)
$$

Substituting $p\left(\mathbf{o}_{1: T}, h_{1: T} \mid \Theta\right)$ in the $Q$ function with the likelihood expression in Equation 6.5, gives us the expanded $Q$ function in Equation 6.7. From this expansion we can conclude that the individual terms do not interact, thus they can be maximised separately.

$$
\begin{gathered}
Q\left(\Theta, \Theta^{\prime}\right)=\sum_{h_{1: T} \in \mathcal{H}} p\left(\mathbf{o}_{1: T}, h_{1: T} \mid \Theta^{\prime}\right) \log p\left(\mathbf{o}_{1: T}, h_{1: T} \mid \Theta\right)= \\
\sum_{h_{1: T} \in \mathcal{H}} p\left(\mathbf{o}_{1: T}, h_{1: T} \mid \Theta^{\prime}\right) \log \pi_{h_{1}}+ \\
\sum_{h_{1: T} \in \mathcal{H}} p\left(\mathbf{o}_{1: T}, h_{1: T} \mid \Theta^{\prime}\right) \sum_{t=2}^{T} \log a_{h_{t-1}, h_{t}, z_{t-1}}+ \\
\sum_{h_{1: T} \in \mathcal{H}} p\left(\mathbf{o}_{1: T}, h_{1: T} \mid \Theta^{\prime}\right) \sum_{t=1}^{T} \sum_{i=1}^{M} \log b_{h_{t}}^{i}\left(\mathbf{o}_{t}\right)
\end{gathered}
$$

The derivation of which values for the individual terms that maximise the $Q$ function is relatively lengthy. We therefore defer all details to Appendix $D$, and here only account for the results of the derivation and show how to compute the necessary quantities. 


\subsubsection{Estimating new parameters}

Computing new parameters $\pi_{i}$ for the initial hidden state distribution that maximise the $Q$ function is done according to Equation 6.8. Here we are taking the conditional probability of each possible state $N$ given the observed data and the current parameters $\Theta^{\prime}$.

$$
\pi_{i}=\frac{p\left(\mathbf{o}_{1: T}, h_{1}=i \mid \Theta^{\prime}\right)}{p\left(\mathbf{o}_{1: T} \mid \Theta^{\prime}\right)}
$$

The new parameters $a_{i j k}$ can be computed using Equation 6.9, where we use $\delta\left(z_{t-1}=k\right)$ to represent the Kronecker delta which is unity when $z_{t-1}$ takes on value $k$, and zero otherwise. Essentially, we are taking into consideration the expected number of times that we have observed a transition from state $i$ to $j$ when $z$ took value $k$, divided by the expected number of times we have seen transitions away from $i$ when $z$ took value $k$.

$$
a_{i j k}=\frac{\sum_{t=2}^{T} p\left(\mathbf{o}_{1: T}, h_{t-1}=i, h_{t}=j \mid \Theta^{\prime}\right) \delta\left(z_{t-1}=k\right)}{\sum_{t=2}^{T} p\left(\mathbf{o}_{1: T}, h_{t-1}=i \mid \Theta^{\prime}\right) \delta\left(z_{t-1}=k\right)}
$$

The final set of parameters that we shall compute to maximise $Q$ are the parameters of the distributions over the observed variables. We let $b_{j k l}^{i}$ denote the parameter of the distribution for observable variable $i$ when it takes on value $l$, given the hidden state $j$ and its $k$ :th parent configuration. An observation $\mathbf{o}_{t}$ will identify one such parameter for each observable variable under a specific hidden state. We let $\delta\left(\mathbf{o}_{t}, b_{j k l}^{i}\right)$ represent the Kronecker delta such that it is unity when the parameter identified by $\mathbf{o}_{t}$ given $h_{t}=j$ is $b_{j k l}^{i}$, and zero otherwise, and likewise let $\delta\left(\mathbf{o}_{t}, b_{j k}^{i}\right)$ be unity when the $k$ :th parent set is identified given hidden state $j$ (regardless of the value of $l$ ). We can then compute each $b_{j k l}^{i}$ such that $Q$ is maximised using Equation 6.10. This can again be seen as dividing the number of times that we expect to encounter a certain event $(j, k, l)$ with the number of times we expect to encounter a superset of these events $(j, k)$.

$$
b_{j k l}^{i}=\frac{\sum_{t=1}^{T} p\left(\mathbf{o}_{1: T}, h_{t}=j \mid \Theta^{\prime}\right) \delta\left(\mathbf{o}_{t}, b_{j k l}^{i}\right)}{\sum_{t=1}^{T} p\left(\mathbf{o}_{1: T}, h_{t}=j \mid \Theta^{\prime}\right) \delta\left(\mathbf{o}_{t}, b_{j k}^{i}\right)}
$$

\subsubsection{Computing necessary quantities}

While Equation 6.8, 6.9 and 6.10 describe which quantities are needed to compute the values necessary to maximise $Q$, the calculation of these quantities are not immediately available. In this section we turn our attention to the 
computation of these necessary quantities. As before, we defer some of the details to Appendix $\mathrm{D}$ and here offer the results from the derivation.

The two quantities that we require, which we shall call $\gamma$ and $\xi$, are presented and expanded in Equation 6.11 and 6.12 Apart from the quantities $\alpha$ and $\beta$, the expansions consists of known quantities (readily available from the model under parameters $\Theta^{\prime}$ ).

$$
\begin{gathered}
\gamma_{j}(t)=p\left(\mathbf{o}_{1: T}, h_{t}=j \mid \Theta^{\prime}\right)= \\
p\left(\mathbf{o}_{t+1: T} \mid \mathbf{o}_{t}, h_{t}=j, \Theta^{\prime}\right) p\left(\mathbf{o}_{1: t}, h_{t}=j \mid \Theta^{\prime}\right)= \\
\beta_{j}(t) \alpha_{j}(t) \\
\xi_{i j}(t)=p\left(\mathbf{o}_{1: T}, h_{t-1}=i, h_{t}=j \mid \Theta^{\prime}\right)= \\
p\left(\mathbf{o}_{t+1: T} \mid \mathbf{o}_{t}, h_{t}=j, \Theta^{\prime}\right) p\left(\mathbf{o}_{t} \mid h_{t}=j, \Theta^{\prime}\right) \times \\
p\left(h_{t}=j \mid \mathbf{o}_{t-1}, h_{t-1}=i, \Theta^{\prime}\right) p\left(\mathbf{o}_{1: t-1}, h_{t-1}=i \mid \Theta^{\prime}\right)= \\
\beta_{j}(t) \prod_{k=1}^{M} b_{j}^{k}\left(\mathbf{o}_{t}\right) a_{i j z_{t-1}} \alpha_{i}(t-1)
\end{gathered}
$$

What is left to do is to define recursively $\alpha$ and $\beta$, and then all required quantities are either already available or computable. We finish this section by defining these two quantities in Equation 6.13 and 6.14

$$
\begin{gathered}
\alpha_{j}(t)=p\left(\mathbf{o}_{1: t}, h_{t}=j \mid \Theta^{\prime}\right)=\prod_{k=1}^{M} b_{j}^{k}\left(\mathbf{o}_{t}\right) \sum_{i=1}^{N} a_{i j z_{t-1}} \alpha_{i}(t-1) \\
\beta_{j}(t)=p\left(\mathbf{o}_{t+1: T}\left|\mathbf{o}_{t}, h_{t}=j\right| \Theta^{\prime}\right)=\sum_{i=1}^{N} \beta_{i}(t+1) \prod_{k=1}^{M} b_{i}^{k}\left(\mathbf{o}_{t+1}\right) a_{j i z_{t}}
\end{gathered}
$$

Note that the equations given here are slightly different from those used when estimating the parameters of a traditional HMM. In Equation 6.9 we are only considering cases under different values of the $Z$ variable, and in Equation 6.10 we are considering different parent configurations rather than just the hidden state. Also, the definition of $\beta$ in Equation 6.14 includes conditioning on $\mathbf{o}_{t}$, since the $Z$ variable at time $t$ may influence the hidden state at $t+1$.

The only part that is left to take into consideration is how we find the parent sets of each observable variable within each hidden state, i.e. how do we learn the structure of the BNs. We shall take this into consideration in the next section, and then move on to synthetic and real-world experiments. 


\subsubsection{Structure learning}

Taking the approach described in [106], we wish to identify the model over the observable variables that, together with the parameters, maximises the last term of Equation 6.7. We shall again resort to the greedy thick thinning algorithm described in Section 2.1.3 to identify the structure over the observed variables, such that the term over the observable variables is maximised in Equation 6.7. Thus within each iteration of the EM algorithm, we also heuristically identify the best structure over the observed variables within each regime.

\subsection{Synthetic experiments}

We shall in this section account for our experiments using synthetic data to compare the GBN-HMM with three other models. The comparison models are: the standard HMM with observation variables that are independent of each other given the hidden state, the SDO-HMM proposed in [102], where observations are again independent given the hidden state, but where we have (using our term) a $Z$ variable, and finally a version of our GBN-HMM but without the $Z$ variable, which we shall call MULTI-HMM (due to their relationship to Bayesian multinets).

\subsubsection{Methodology and data generation}

A single sample was generated as follows (with input to the procedure the predictive power of the $Z$ variable).

Four BNs were created by using the method proposed in [107] to generate DAGs uniformly at random over four random variables. We then uniformly at random generated parameters for the resulting conditional distributions, using the method described in [108]. The number of states for each variable was determined uniformly between two and five, except for the $Z$ variable which was given four states.

The first data point in the sample was generated from the first BN. The value of the $Z$ variable then determined which $B N$ to take the second data point from, with a certain level of predictiveness (the supplied predictive power). For instance, if the $Z$ variable took value two, and the predictive power was 0.6 , then there was a $60 \%$ chance that the next data point would come from the second $\mathrm{BN}$, and a $40 \%$ chance that the next data point would come from the same $\mathrm{BN}$ as the previous data point. That is, with probability 
0.4 we have $H_{t}=H_{t-1}$ and with probability 0.6 we have $H_{t}=Z_{t-1}$. We repeated this until there were 1000 data points in the sample.

Following this procedure we generated 50 samples for each of the predictive powers $0.6,0.7,0.8$ and 0.9 .

For the synthetic experiments we were interested in how well the models fit held out test data. Therefore, for each sample, we employed a 5-fold crossvalidation procedure using two thirds of the data to determine the number of hidden states, estimate the parameters of the models, and to learn the BN structures for GBN-HMM and MULTI-HMM. For SDO-HMM and GBNHMM the models were told which $Z$ variable to use. The remaining third was treated as held out test data, the likelihood of which will be reported.

\subsubsection{Results and discussion}

In Table 6.1 the results from the synthetic experiments are reported. Each row represents a certain predictive power. The values in the table are the means of the log-likelihoods of the held out test data over the 50 samples, given each model.

Already when the $Z$ variable has a predictive power of 0.6 , the GBNHMM had a considerably better fit to the data than both HMM and MULTIHMM (note that this is log-scale). However, the SDO-HMM was also able to utilise this predictive power to get a similar fit as the GBN-HMM. As the predictive power of the $Z$ variable increased to 0.7 , the difference between the GBN-HMMs' fit of the data and the other models increased, suggesting that taking this predictiveness into account, and allowing for multiple BNs, can improve the appropriateness. When we look at the outcomes when the predictive power was increased to 0.8 and 0.9 , the two models that do not utilise a $Z$ variable (HMM and MULTI-HMM) drift further from the GBNHMM, while the HMM-SDO reversed and came closer again. Although the GBN-HMM outperforms the other models throughout all experiments, it is interesting to see that the SDO-HMM can outperform HMM and MULTIHMM by utilising the $Z$ variables predictive power.

While the experiments that we have reported in this section work well as a confirmation of the proposed model's appropriateness, we shall now turn our attention to experiments where we wish to employ the model for a specific task. We shall again consider a situation where we wish to trade shares on a stock market, exploring and comparing the performance of the four models discussed. 
Table 6.1: Means of log-likelihoods of held out data, using different predictive powers of the $Z$ variable.

\begin{tabular}{|c|c|c|c|c|}
\hline & HMM & SDO-HMM & MULTI-HMM & GBN-HMM \\
\hline Predictive power $=0.6$ & -1546.438 & -1537.453 & -1558.732 & $\mathbf{- 1 5 3 6 . 5 2 9}$ \\
\hline Predictive power $=0.7$ & -1538.541 & -1526.940 & -1550.820 & $\mathbf{- 1 5 1 8 . 5 9 0}$ \\
\hline Predictive power $=0.8$ & -1535.269 & -1509.830 & -1546.112 & $\mathbf{- 1 5 0 6 . 7 2 6}$ \\
\hline Predictive power $=0.9$ & -1513.058 & -1476.529 & -1526.843 & $\mathbf{- 1 4 7 5 . 4 3 6}$ \\
\hline
\end{tabular}

\subsection{Stock market trading revisited}

In this section we shall revisit the algorithmic trading application first introduced in Chapter 3 . We will again use technical analysis indicators as random variables in our models, and measure the Sharpe ratio using each of the models discussed in this chapter.

\subsubsection{Methodology}

We used the MACD and RSI indicators (please see Section 3.1.3 and Equations 3.1 and 3.2, which gave us two observable variables in our models. We additionally considered the first order backward difference of these variables (i.e. we approximated the indicators' first order derivatives), giving us a total of four observed variables. The MACD was discretised into two states, positive and negative, and used as the $Z$ variable. The rest of the indicators were discretised into four states, using their respective mean and one standard deviation below and above their mean as cut points.

We used the same data as in Section 3.3, i.e. daily data between 200301-01 and 2012-12-31 for seven actively traded stocks: AAPL, AMZN, IBM, MSFT, RHT, NVDA and GE. To create multiple simulations from this data we divided the data into ten blocks (one year per block), and created seven simulations by first using block one, two and three as training data and block four as testing data, and then block two, three and four as training data and block five as testing data, and so on (the same routine as was described in Section 3.2.2.

As in the synthetic experiments in Section 6.4, we employed a 5-fold cross-validation procedure using the training data to decide upon the number of hidden states, the parameters of the models, and the BN structures within the GBN-HMMs and MULTI-HMMs. For SDO-HMM and GBN-HMM the models were told to use the MACD variable as the $Z$ variable.

While one's first intuition may be to attempt to label the hidden states of our models as "buy", "sell", etc. and thereby generate signals that can be 
Table 6.2: Annual Sharpe ratio comparison.

\begin{tabular}{|l|c|c|c|c|}
\hline & HMM & SDO-HMM & MULTI-HMM & GBN-HMM \\
\hline Apple (AAPL) & 0.844 & 0.708 & $\mathbf{0 . 8 4 9}$ & 0.718 \\
\hline Amazon (AMZN) & 0.466 & 0.580 & 0.449 & $\mathbf{0 . 5 9 2}$ \\
\hline IBM (IBM) & $\mathbf{0 . 7 1 3}$ & 0.521 & 0.699 & 0.616 \\
\hline Microsoft (MSFT) & 0.091 & -0.189 & -0.307 & $\mathbf{0 . 2 1 9}$ \\
\hline Red Hat (RHT) & -0.198 & $\mathbf{0 . 1 1 1}$ & -0.780 & -0.085 \\
\hline Nvidia (NVDA) & 0.113 & 0.211 & 0.262 & $\mathbf{0 . 3 0 8}$ \\
\hline General Electric (GE) & 0.062 & 0.362 & -0.378 & $\mathbf{0 . 4 1 9}$ \\
\hline
\end{tabular}

executed, this is not the approach we will take in this application. We do not know how many hidden states will be identified in each simulation, thus it would require some automatic labelling based on the number of states and historical advantage of different types of labelling. Instead, we shall build our rules as follows:

- On day $t$, when we know the values of $\mathbf{O}_{1: t}$, we shall make a prediction of the MACD variable at time $t+1$.

- If $\mathrm{p}\left(M A C D_{t+1}=\right.$ positive $\left.\mid \mathbf{O}_{1: t}\right)>\theta$, then generate a buy signal.

- If $\mathrm{p}\left(M A C D_{t+1}=\right.$ negative $\left.\mid \mathbf{O}_{1: t}\right)>\theta$, then generate a sell signal.

We are thus generating buy/sell signals when enough of the probability mass indicates that the MACD is positive/negative. The particular $\theta$ used was determined for each model by generating trade signals using the training data. For each simulation we generated signals for each block reserved for training (three blocks per simulation) and calculated the Sharpe ratio per block using different $\theta(0.50,0.55, \ldots, 0.90,0.95)$. The $\theta$ used on the test data was then the $\theta$ with the highest average Sharpe ratio over the training blocks.

\subsubsection{Results and discussion}

Signals were generated for each held out test block, and the annual return and standard deviation was calculated for each block and model, giving rise to an annual Sharpe ratio for each model and traded stock. The annual Sharpe ratios are given in Table 6.2 .

From the table we can see that the use of multiple BNs (i.e. MULTIHMM and GBN-HMM) yields a higher annual Sharpe ratio for five of the seven stocks, losing out to SDO-HMM for RHT and HMM for IBM. In four out of the five cases where using multiple BNs was better, the GBN-HMM outperformed the MULTI-HMM. Thus in general, allowing for multiple BNs 
over the observable variables does increase the performance of the trading systems. Similarly, when considering the models that include a $Z$ variable against those which did not, we see that the $Z$ variable models won five against two. When comparing the use of both multiple BNs and a $Z$ variable, i.e. the GBN-HMM, the outcome is four against three in favour of the GBN-HMM. So even when all the other models are counted as one, the GBN-HMM wins. It should be noted that the models are all generative 1 thus they have been learnt with the goal of explaining the data generating process, and not to the specific task of stock trading. The case of SDO-HMM outperforming GBN-HMM on RHT is evidence of this difference between goals, as the GBN-HMM should always explain the data better, or the same, as the SDO-HMM, as the former is capable of mimicking the same structure as the latter.

It seems that the different models are advantageous under different circumstance, although the GBN-HMM seems to have an advantage in general. However, since GBN-HMMs embrace the other three models, we could take the structure learning further than only for the individual BNs, and learn which one of the four models considered is the most appropriate for the current task. We however leave such exploration to future work.

\subsection{Summary}

The GBN-HMM model proposed and discussed in this chapter is not meant to replace or compete with the GBN model that we have introduced and developed throughout Chapters 2 through 4 . We emphasise that HMMs are mixture models and GBNs are suited for those cases where mixtures are not wanted. Instead, we hope that the model we have proposed and shown the efficacy of in this chapter can be of use in applications where HMMs are already in practice. We imagine that the assumption of independence among the observed variables given the hidden variable is often made out of convenience, rather than from strong evidence that such is the case. Furthermore, while the observed variables in a traditional HMM do affect the distribution of the next hidden state, this influence is through the current hidden state, rather than direct. As we have seen in the experiments in this chapter, allowing for this direct influence may improve the performance of the model on the task at hand, but may also allow for a more appropriate model from a generative perspective.

\footnotetext{
${ }^{1}$ Except for the threshold $\theta$ which was fixed using the Sharpe ratio.
} 
In Chapter 8 we shall attempt to summarise the research contained within this thesis, and offer some ideas of possible future work. Before doing so we wish to return to GBNs, however this time from a causal perspective. Instead of considering only BNs as components in GBNs, and using them as inference machines, we shall be more general and allow any representation of independence models (still connected using gates). We shall refer to these as gated models, preserving the semantics and graphical representation of GBNs when possible. 


\section{Chapter 7 \\ Modelling causal scenarios using gated models}

Already in Chapter 2 we mentioned how CSIs can be used to improve upon the efficiency of modelling and inference in probabilistic graphical models. We also mentioned that we would have reason to continue our discussion of CSIs, and so in this second to last chapter we shall discuss how we can exploit CSIs in a causal framework, allowing us to define a gated model, which may allow us to identify more causal effects than would be possible using a single causal graph. A gated model is essentially a GBN but where we allow the BNs to be replaced with any representation of an independence model. We shall see how our gated models can be used to model certain causal phenomena that may occur due to CSIs, such as unstable and non-deterministic effects of interventions.

Note, although we have already introduced and defined GBNs in Section 2.2, we shall in Section 7.3 , for the sake of clarity introduce the building blocks that we will use in order to create gated models. We shall also introduce new criteria that we can use within the trigger logic of our gates. Before we define gated models we shall look at how they can be used to model CSIs in BNs in Section 7.1 and in causal models in Section 7.2. Before moving on to the final chapter of this thesis we shall in Section 7.4 look at causal phenomena that may occur due to CSIs, and how they can be modelled using gated models. 


\subsection{Context specific independence in Bayesian networks}

In a BN, an edge between two nodes should be present if there is a potential direct association between the two variables that the nodes represent, even if this association is only present for a certain range of the values the variables may take. Studying the conditional probability distributions that are associated with a BN may however reveal that some edges can be removed when the parent variables take on certain values.

Consider the DAG representation of a BN in Figure 7.1a, and the additional knowledge that the edge $X \rightarrow Z$ is unnecessary when $X>0$. This CSI is obviously not revealed by the DAG in the figure. An alternative way of representing this BN is to use a gated model, an example of which is depicted in Figure 7.1b (in this case the contained models are BNs, thus it is a GBN, however we wish to make a point in this chapter that we can include any independence model representation). In Figure $7.1 \mathrm{~b}$ we have two BNs connected with gates, when $X$ takes a value greater than 0 we shall use the $\mathrm{BN}$ to the right, where there is no edge between $X$ and $Z$, and when $X$ is less than or equal to 0 then we shall use the BN to the left (where the edge is present). We have here implicitly introduced a new type of criteria that can be used in the trigger logic of gates, a statement about the value of a random variable.

The gated model representation allows us to not only reduce the number of edges in some circumstances (which in turn may imply less computation), but also graphically state the CSIs that exist among the variables.

In some cases we may have CSIs that are due to unmodelled and unobserved variables. Consider the DAG representation of a BN in Figure 7.1c, where we assume that $U_{1}$ and $U_{2}$ are unobserved. If the edge between $Z$ and $U_{1}$ disappears when $U_{2}>0$, then in a data set over $X, Y$ and $Z$ there will be data points for which the edge $Z \rightarrow Y$ has to be present, and data points for which it is not required, however it may not be evident from the conditional distributions estimated from the data set. For instance, assume that $X$ and $U_{2}$ both follow standard normal distributions, and that the conditional distributions of $Y, Z$ and $U_{1}$ are the sum of their parents with some additional noise. We generate data for the variables $X, Y$ and $Z$ under both the condition that $U_{2} \leqslant 0$, and the condition that $U_{2}>0$. For some of the generated data points, the distribution of $U_{1}$ is going to be the sum of $Z$ and $U_{2}$, whilst for others it is going to be only dependent on $U_{2}$ (recall that $Z \rightarrow U_{1}$ disappears when $U_{2}>0$ ). Likewise, the value of $Y$ is going to (indirectly) depend on 


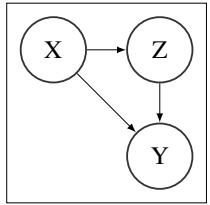

(a)

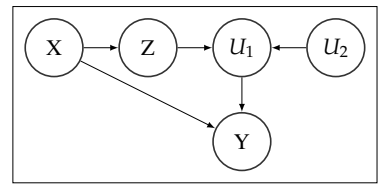

(c)

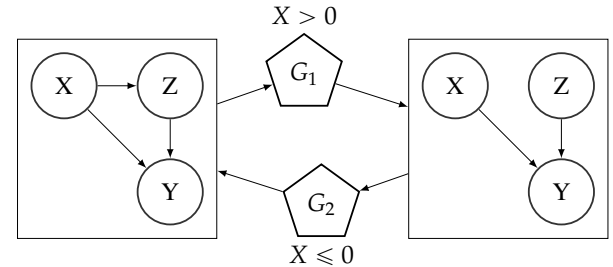

(b)

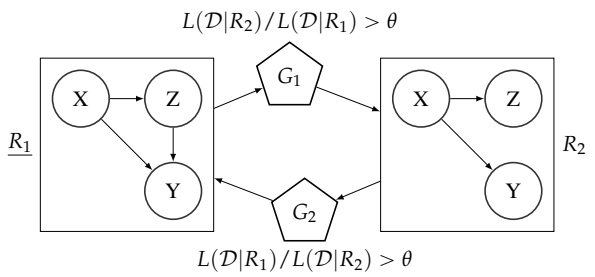

(d)

Figure 7.1: In (a), a single graph that does not convey that $X \rightarrow Z$ can be removed if $X>0$, a gated model representing this extra knowledge is given in (b). When the CSI is dependent on unmodelled variables, $U_{1}$ and $U_{2}$ in (c), we cannot discern context based on variables taking specific values. The gated model in (d) uses threshold gates to decide which model is appropriate.

the value of $Z$ for some data points, but not all. However, there is no specific value of $Z$ (nor $X$ ) that can inform us of when $Y$ should depend on $Z$ or not. We can therefore not create gates which specify for which values of any of the observed variables we can remove $Z \rightarrow Y$. In such cases we use a gate which judges which graph is more appropriate given some data $\mathcal{D}$. In Chapter 4 appropriateness was measured using the likelihood of the data given the model. We will continue to use the likelihood in our examples, which we shall denote with $L$ in our graphical representations. In Figure 7.1d, where we have labeled the graphs $R_{1}$ and $R_{2}$, we express such conditions using a gated model. The model makes it clear that it is necessary to continually assess whether or not the other graph is more appropriate (above some threshold $\theta)$.

\subsection{Context specific independence in causal models}

In addition to being conveyors of independence models, BNs have also been used as causal models. Judea Pearl has developed sound and complete criteria to identify causal effects [11]. In this context, identify refers to computing 


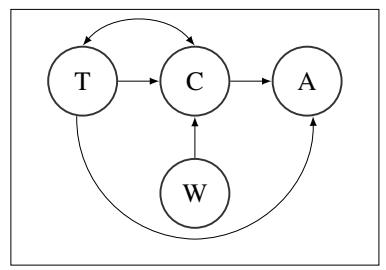

(a)

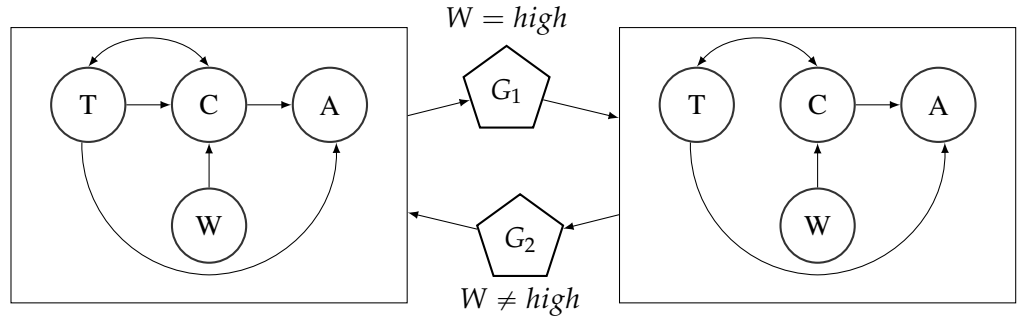

(b)

Figure 7.2: In (a), the effect $p(A \mid d o(T))$ is not identifiable due to confounding between $T$ and $C$. In (b), in the context where $W=$ high, the effect $p(A \mid$ do $(T))$ is identifiable.

from observed quantities and causal effect refers to the distribution over a variable given an intervention in another. Note that this is different than observing a variable in some state, as intervening means that we change the natural causal mechanism of some variable, externally setting them to some specific value. One of the criteria developed by Pearl is graphical, called Q-decomposition, and the other is algebraic, also known as do-calculus.

In a causal graph an edge must be present if there is a potential direct causal relationship between the variables, even if this relationship is only present for a range of the values the variables may take. While being able to remove an edge in a $\mathrm{BN}$ which defines associational relationships may result in faster computation and easier estimation of parameters, removing an edge in a causal graph may lead to causal effects being identifiable that previously were not.

Consider the acyclic directed mixed graph (ADMG) in Figure 7.2a In this type of causal graph we use directed edges for direct causal relationships, bidirected edges indicate hidden common causes (also known as confounding effects), and no directed cycles are allowed. Due to confounding between $T$ and $C$, depicted with a bidirected edge, we cannot identify the causal effect $p(A \mid d o(T))$. That is, since there exists a so-called bow pattern $T \rightarrow C \rightleftarrows T$ 
[11], the correlation between $C$ and $T$ can arbitrarily be split between the causal relationship $T \rightarrow C$ and the confounding relationship $T \rightleftarrows C$. So there is no way to discern from observational data alone how much is due to one and how much is due to the other.

However, if we could identify a CSI, for instance when $W=$ high the edge $T \rightarrow C$ can be removed, then the effect $p(A \mid d o(T))$ would be identifiable in this specific context (in particular we would have $p(A \mid$ do $(T))=$ $p(A \mid T)$ ). The ADMG in Figure $7.2 \mathrm{a}$ however lacks the ability to directly tell us that $p(A \mid d o(T))$ is identifiable in the context of $W=$ high. There are two aspects that surfaces that a gated model can address. First, examining the gated model in Figure 7.2b, we can directly read off the graph which independencies hold in the different contexts using the d-separation criteria for ADMGs, and we can use $d o$-calculus to decide which causal effects are identifiable from observational data (within the two contexts). Second, the gated model is explicit about the order in which certain conditions must be met, i.e. $p(A \mid d o(T))$ is not identifiable until we have entered the context where $W=$ high . Thus the graph may change between different contexts, and we need the changed graph in order to use $d o$-calculus.

Following the same reasoning as with BNs, even when the context in which certain independencies exist cannot be discerned from modelled variables, we can create gated models that can identify the causal graph which is presently most appropriate, and use this graph to decide which causal effects are identifiable.

In summary, we face a modelling task where CSIs may be present, either due to some relationship among the modelled variables or involving unmodelled variables. Therefore, we suggest that we cannot consider a single causal graph alone, but must instead consider an entire causal scenario. We shall introduce gated models as a rich language for expressing such causal scenarios, and use them to decide which contained graph should be used for inference purposes.

\subsection{Gated models}

A gated model $M$ is a triple $<\mathbf{R}, \mathbf{G}, \mathbf{E}>$, where $\mathbf{R}$ is a set of regime models, $\mathbf{G}$ is a set of gates and $\mathbf{E}$ is a set of directed edges connecting the regime models in $\mathbf{R}$ with the gates in $\mathbf{G}$. Each regime model in $\mathbf{R}$ is a collection of conditional independence statements among the modelled variables. When possible, these statements may be visualised using a graph, for instance a DAG. We will use $R$ to label regimes (indexing them with subscripts when 


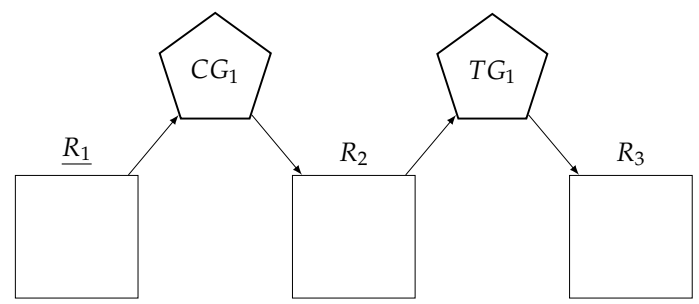

Figure 7.3: Example of a gated model.

necessary). In the following discussion we will use the example of a gated model offered in Figure 7.3. In this figure, regime models are depicted as squares (labelled with $R_{1}, R_{2}$ and $R_{3}$ ), while $C G_{1}$ and $T G_{1}$ represent gates connected with the regime models using the directed edges of $\mathbf{E}$. The label $R_{1}$ is underlined to emphasise that it is the initial regime that we consider.

\subsubsection{Gates}

The structure of the gated model $M$ defines which regime models can transfer into each other, while the gates define how these transitions can be made. A gate holds a condition that must be fulfilled in order for a regime transition to occur through the gate. For the gated models that we will use in this chapter we shall use the following conditions:

- A context, e.g. the condition for moving from $R_{1}$ to $R_{2}$ via $C G_{1}$ in Figure 7.3 is that some modelled variable takes some specific value, thereby setting a context. We use $C G$ to represent gates with context conditions. Both gates in the gated model in Figure $7.1 \mathrm{~b}$ are context gates.

- A threshold that is broken, e.g. moving from $R_{2}$ to $R_{3}$ via $T G_{1}$ in Figure 7.3 requires that the likelihood $L\left(\mathcal{D} \mid R_{3}\right)$, given some data $\mathcal{D}$, is greater than $L\left(\mathcal{D} \mid R_{2}\right)$ with some threshold $\theta$. Both gates in the gated model in Figure $7.1 \mathrm{~d}$ are threshold gates.

- A combination of a context and a broken threshold. We shall use CTG to represent gates with combined conditions. We shall see examples of combination gates in the coming sections. 


\subsubsection{Setting a context via conditioning or via intervention}

Consider the gated model in Figure $7.1 \mathrm{~b}$. We know that we should move from the left model to the right model if $X>0$, thus if we ask the probabilistic query $p(Y \mid X=5)$, then we should use the model on the right hand side, condition on $X$ and marginalise $Z$. The question we have asked is "what is the distribution over $Y$ for those units that have $X=5$ ". We might however ask the causal query $p(Y \mid \operatorname{do}(X=5))$, which we in this case should treat likewise as with the probabilistic query, yet the question we have asked is "what is the distribution over $Y$ if we force all units to have $X=5$ ". In both cases we are setting a context, and the gated model does not need to distinguish between them, however one must take care to use the correct algebra when answering queries. In light of this discussion, we shall not distinguish between setting the context by conditioning or intervening, and instead use the concept that suits the narrative best. However, we keep in mind that if we wanted to go further and compute actual quantities, we must use the correct algebra.

\subsubsection{Execution and learning}

Having specified a gated model we are naturally interested in using the model in a particular situation. The algorithm described in Section 2.2.3 can be used to execute the gated model (with the addition of checking for the newly introduced context condition in the gates). Naturally, we assume that the quantities required to check the trigger logic of the contained gates are computable (e.g. likelihood ratios).

The two algorithms that we have introduced in Chapters 4 and 5 could potentially be used for learning gated models. In those chapters we did not attempt to explain why the independence models changed among regimes, however one possible explanation for the changes could be CSIs involving unobserved variables. The algorithms that we have proposed do not explicitly target identification of CSIs, so we do not want to go so far to say that the algorithms learn gated models in the sense that they represent regimes due to CSI. While this is a very interesting question, we will leave this line of research for future work.

\subsection{Modelling causal scenarios using gated models}

In Section 7.2, we touched upon the subject of using gated models to express more richer causal scenarios, rather than a single graph that represents a static context. In this section we shall offer four causal scenarios that can be better 


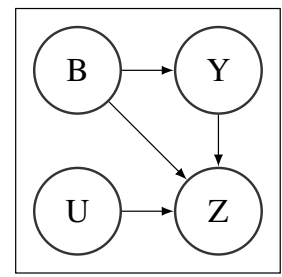

Figure 7.4: A single causal graph cannot capture the extra knowledge regarding CSIs in the blood pressure example.

modelled using gated models since they contain CSIs. These examples aim to highlight the expressive power of gated models. The first two concerns situations where the effect of an intervention may not be stable and where the outcome of the intervention may not be deterministic. The third example requires us to stretch somewhat the definition of a context, but will allow us to model causal scenarios where the effects of interventions may be different depending on how the intervention is applied. After having discussed these three examples, we shall describe an example which shows how using gated models allows us to identify causal effects that cannot be identified using a single graph.

\subsubsection{Unstable effect and nondeterministic outcome of interventions}

Assume that low blood pressure puts the human body in a context where it goes back and forth between a physiological stable state and an unstable crisis state. When low blood pressure occurs we know that the body enters the physiological stable state, but may then go back and forth between the stable and crisis state. If the blood pressure returns to normal, this instability disappears.

In Figure 7.4, a single causal graph for this scenario is depicted (we let $B$ represent blood pressure and $U, Y$ and $Z$ some physiological measurements). Although we shall let $U$ be unmodelled (and unobserved) in our gated models, we have included it in the causal graph in Figure 7.4 (i.e. this is a representation of the true underlying causal structure). Additionally, we know that the edge $B \rightarrow Y$ is lost in the context of $B=$ low, and $Y \rightarrow Z$ is lost when $B=$ low and $U>0$.

The single causal graph, although it represents the true causal structure with $U$ included, tells us very little about the narrative given at the beginning of this section. However, a gated model can communicate the additional 


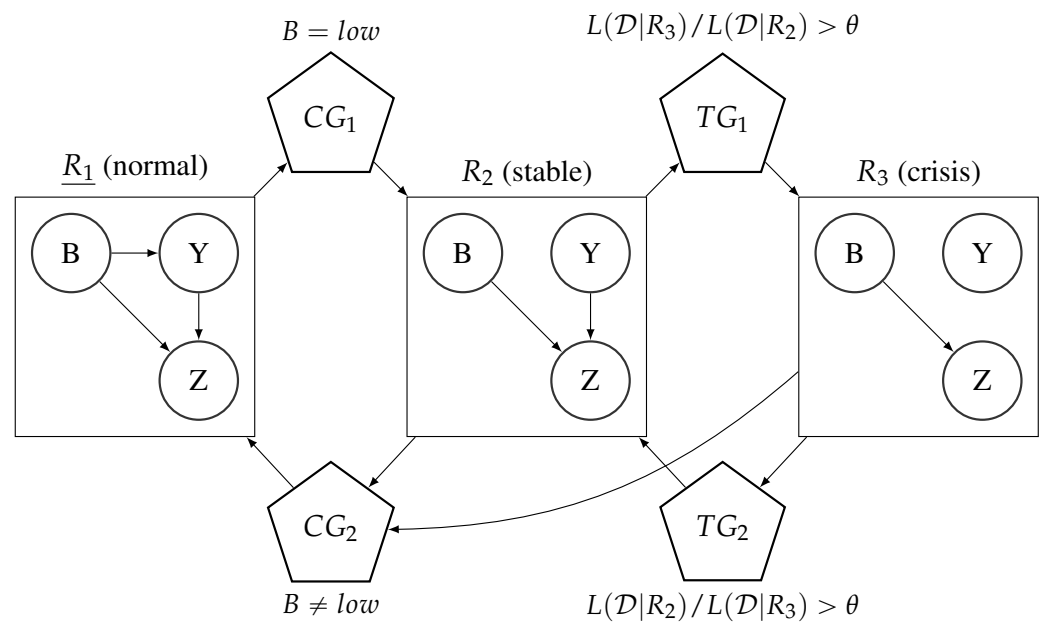

Figure 7.5: The immediate effect of low blood pressure is a move to a physiological stable state, but may lead to transitions back and forth with a crisis state.

knowledge regarding the CSIs. The resulting gated model is depicted in Figure 7.5. As we can see, once the blood pressure $(B)$ goes low, we change from $R_{1}$ to $R_{2}$, the physiological stable state under low blood pressure. Since $U$ is unmodelled, we cannot use a context gate to describe when we should switch between $R_{2}$ and $R_{3}$ (the crisis state), thus we need to use a threshold gate that can identify which of the two regimes is most appropriate. The gated model also makes it clear that increasing the blood pressure while either in the crisis state or the stable state will take the body back to the initial regime.

To demonstrate nondeterministic outcomes of interventions, we shall change the previous scenario slightly. This time, assume that we do not know if we will enter the stable state or the crisis state when the blood pressure is set to low (via some intervention). Furthermore, the stable and crisis states cannot transition into each other, i.e. once the blood pressure turns low, the body enters a stable or crisis state and does not change between them. The single causal graph stays the same (Figure 7.4), and again tells us very little about the scenario. Using a gated model we can model this causal scenario, but this time using combination and context gates, as depicted in Figure 7.6. The gated model tells us that when the blood pressure turns low, we must assess which of the two regimes is most appropriate, and it is the data $\mathcal{D}$ that determines the appropriate regime model (recall that this is due to the CSIs that are created due to the unmodelled variable $U$ ). 


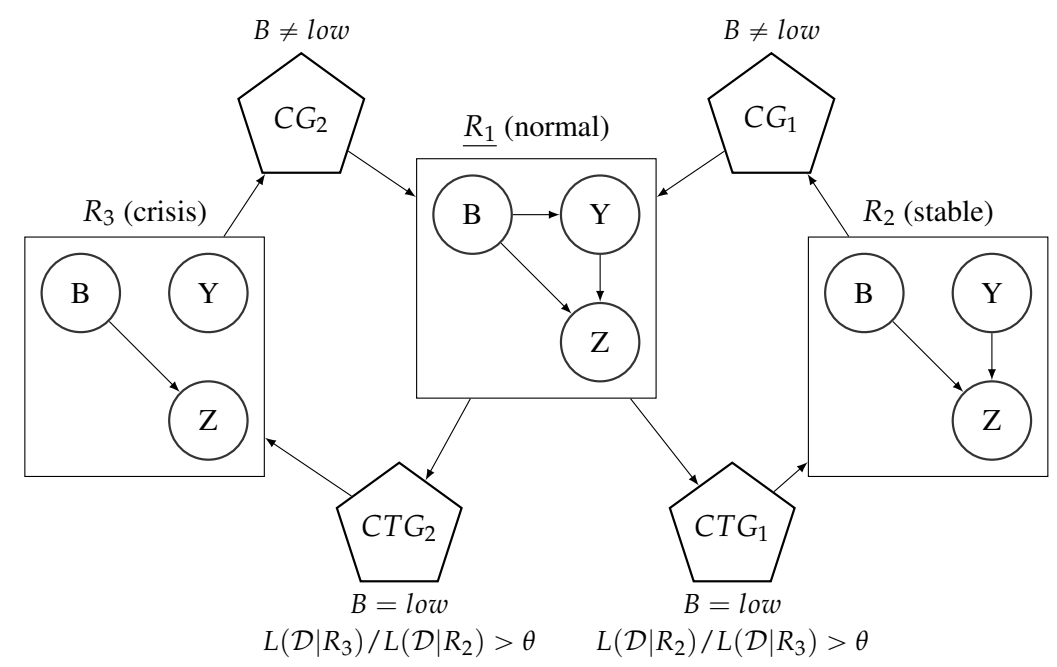

Figure 7.6: The immediate effect of low blood pressure is unknown, it may either lead to the stable or the crisis state.

\subsubsection{Mechanism dependent outcome of interventions}

The third example that we shall describe requires us to expand somewhat the definition of a context. So far we have defined a context as a variable taking on some value. However, we shall now allow for the mechanism used to enter a certain context to be part of the context itself, for instance we may have two different drugs that we can use, both with the same aim, but with different consequences.

Consider the monthly physical activity $(W)$ and monthly alcohol consumption $(C)$ of a person in a population. Assume that there exists a leaflet which contains information about the negative consequences of overconsumption of alcohol, and that each person in the population can read the leaflet or not $(T)$. In general, if the leaflet is read it reduces the amount of alcohol consumed. Assume further that high physical activity can be the result of two different mechanism: either sending a person to bootcamp or motivating them to increase their physical activity on their own. In this scenario, a CSI is introduced such that $T \rightarrow C$ is lost if the person has a high physical activity due to being sent to bootcamp (no alcohol available at the bootcamp, thus reading the leaflet has no effect on alcohol consumption). However, the same is not true for people that are motivated to increase their physical activity on their own, they have access to alcohol, thus reading the leaflet does have some effect. 


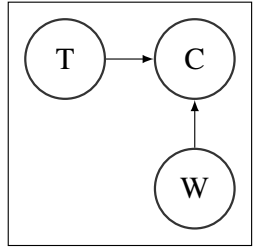

(a)

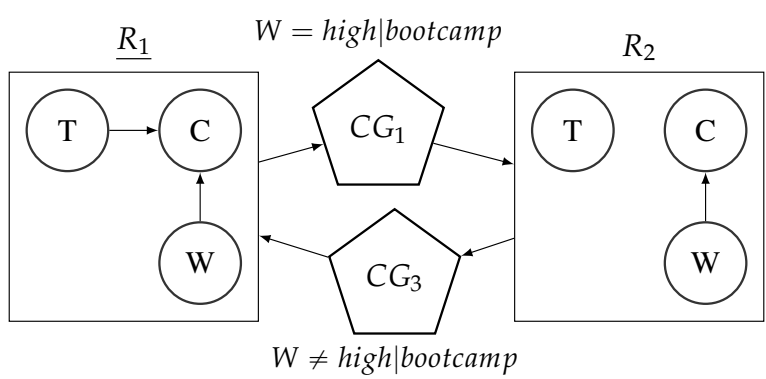

(b)

Figure 7.7: In (a), the single graph does not encode the mechanism dependent context, however in (b) the mechanism used to set the context is part of the context itself, thus different outcomes are achived depending on the mechanism used.

As before, using a single graph (Figure 7.7a) to represent this scenario results in a less informative graph than what we can express with a gated model. In Figure $7.7 \mathrm{~b}$ we have depicted a gated model which can represent such a scenario, where we have used $W=$ high $\mid$ bootcamp to mean that the bootcamp mechanism was used.

\subsubsection{Using gated models to identify causal effects}

In this section we shall expand upon the alcohol and physical activity example given in Section 7.4.2. This time we shall focus on the identification of causal effects within the regimes, and show how gated models allows us to identify effects that cannot be identified using a single graph.

Consider the causal scenario depicted in Figure 7.8, which is now represented using a gated model with ADMGs. Here, $C$ represent the monthly consumption of alcoholic beverages for an individual, $W$ represents the individual's monthly level of physical activity, $A$ represents alcohol related accidents that the individual has been subjected to, and $T$ represents whether or not the individual has read a leaflet containing information about the negative effects of overconsumption of alcoholic beverages, and information about how to avoid accidents when consuming alcohol. From the initial regime $R_{1}$, we can tell that there is confounding between $T$ and $C$. The causal effect $p(A \mid d o(T))$ is therefore not identifiable.

In this scenario, individuals who have a high level of physical activity do not change the number of alcoholic beverages they consume if they read the leaflet. By exploiting this CSI in $R_{2}$, the effect $p(A \mid d o(T))$ can in this 


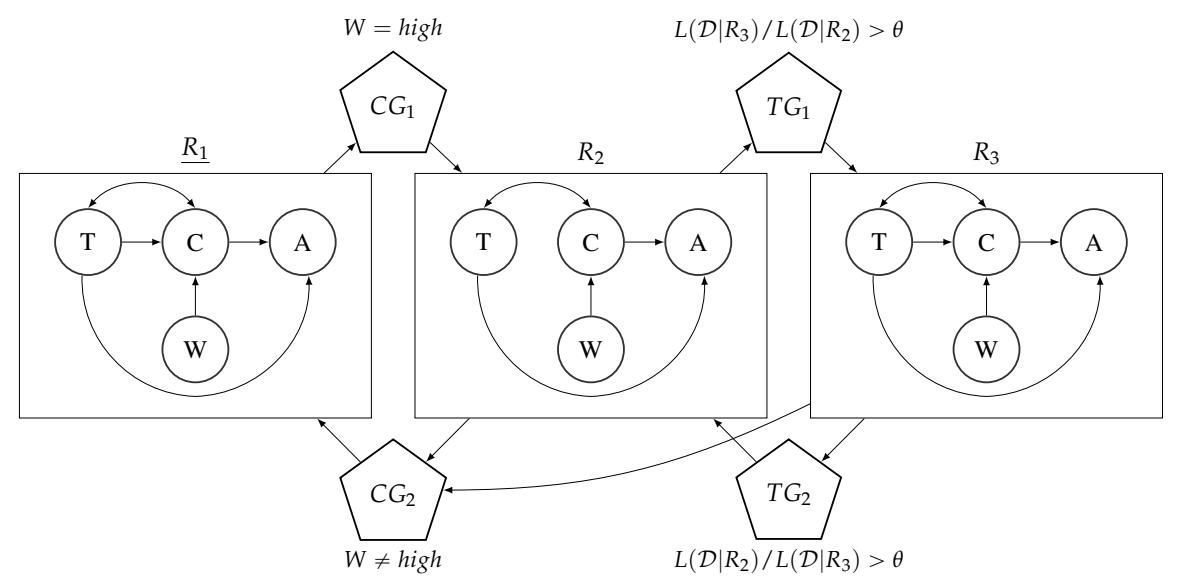

Figure 7.8: A gated model using ADMGs. The causal effect $p(A \mid$ do $(T))$ cannot be identified from observational data in $R_{1}$. However, exploiting certain CSIs the effect is identifiable in the regime model $R_{2}$.

context be identified from observational data, in particular $p(A \mid$ do $(T))=$ $p(A \mid T)$ which can be estimated from observational data. Thus the investigator can collect observational data in this regime, and use do-calculus to calculate $p(A \mid \operatorname{do}(T))$. If the individual no longer maintains a high level of physical activity, then the gated model expresses that such effects no longer can be calculated from observational data. However, the gated model also expresses that the effect of high physical activity is not stable, and that under certain circumstances (due to unmodelled variables, hence the use of threshold gates), the edge $T \rightarrow C$ might be recovered even when $W=$ high. The investigator must therefore be careful, as it may be the case that $R_{3}$ is more appropriate than $R_{2}$, thus the effect would again not be identifiable from observational data. Therefore, as the investigator collects data in the context of $W=$ high it is necessary to assess which of $R_{2}$ and $R_{3}$ is most appropriate.

\subsection{Summary}

The graph centric approach of $d o$-calculus is a powerful machinery for causal inference, allowing us to discern which causal effects can be identified from observational data and which cannot. The approach does however entail a type of worst-case scenario; the graph structure is the source of information regarding which effects are identifiable. This allows the development of causal inference rules to take a non-parametric approach, but does ignore any extra knowledge that may exist regarding the system under observation. In 
this chapter we have discussed how we can exploit knowledge about CSIs among the variables in the system, in such a way that we can identify more causal effects than would be possible using a single graph approach.

The examples that we have discussed touches upon certain phenomena that many practitioners may encounter when dealing with real-world systems, for instance uncertainty in the outcome of interventions and unstable effects. We have taken the approach of explaining such phenomena in terms of CSIs involving both modelled and unmodelled variables. We are however not claiming that CSIs are the true nature of such of phenomena, but rather that it is an operational way of modelling and dealing with such phenomena. The benefit of doing so is that we need not throw out existing frameworks for causal inference, e.g. do-calculus, but can rather keep and extend their usefulness. Furthermore, we need not break the invariance assumption often necessary in causal inference, i.e. if we somehow change the mechanism that determines the value for variable $X$, then this should not change the mechanism that determines the value for another variable $Y$. A gated model could still be used if we wished to break this assumption, we could for instance introduce a gate that had the condition that an intervention must be in place, i.e. conditioning on $W=$ high does not fulfil the condition, yet do $(W=h i g h)$ does. In such cases we may have a very difficult problem when it comes to causal inference, as mechanisms would no longer be modular and transferrable between observation and intervention, yet the model could at least be expressed. 



\section{Chapter 8}

\section{Conclusions and future work}

In this final chapter we shall recapitulate the line of research upon which this thesis is written. By doing so we shall highlight some of the questions that have emerged that may create a foundation for future research.

\subsection{Algorithmic trading}

We have investigated the use of GBNs (and HMM-GBNs) as alpha models in algorithmic trading systems. From the results of the experiments in Sections 3.3 and 3.5 , it is clear that our proposed learning algorithms were able to learn GBNs that in most cases were successful in maintaining similar or better reward than the BaH strategy, but perhaps more importantly our GBNs consistently lowered risks towards invested capital. As mentioned earlier, we would like our reward from investments to be accessible, i.e. we would like a monotonic growth in value. A passive approach such as BaH may imply long periods of drawdown, during which the reward of the investment is not available. We also found that using different BNs in our GBNs resulted in higher Sharpe ratios than using the same BN in all phases.

The learning algorithms presented in Chapter 3 both rely on choices made prior to learning, e.g. choosing which features to include and the structure among these features. Since our learnt GBNs did not improve upon the $\mathrm{BaH}$ strategy with respect to the Sharpe ratio for all stocks and indices considered, we would like to investigate if by avoiding such choices and including them as part of the learning itself we can improve the GBNs performance. We imagine that a learning algorithm with feature selection could choose among several technical analysis indicators to include in the different phases, as well as the parameterisation of these and the structure among them. Also, it would be interesting to combine the learning algorithms we proposed for detecting 
regimes in Chapters 4 and 5 so that we do not have to fix the phase or regime structure of the GBNs before learning.

\subsection{Modelling regimes in data}

The second part of this thesis concerns the identification and prediction of regimes in data. We have defined such regimes as steady states among the random variables, specifically concerning the associational relationships among them. The aim of the approach we took in Chapter 4 was to identify the regime transition structure and use this knowledge to create a GBN that could later be used to detect the current regime in newly collected data. We find this approach very useful for two reasons: we can show which regimes transition into one another, which in and of itself may be useful information, and secondly we can detect when regime changes happen, allowing us to use the most appropriate model. We have shown in Section 4.5 that this approach is useful for baseball managers and coaches to understand how their players' performance change over time, and perhaps allow them to make changes that enforce such changes. In Section 4.6 we used the same approach to detect volatility regimes in financial markets, from which the results clearly show that the learning algorithm is able to capture such regimes following the historical narrative of the time.

In Chapter 5 we began a departure from GBNs to investigate if we could learn the most appropriate $\mathrm{BN}$ in an online fashion, and we found that we could keep the performance of our learnt models constant compared to a single model learnt using all data. The learning algorithm that we proposed does not limit us to learning only BNs, thus we are interested in seeing others adopt the same approach for other (non)graphical models.

In Appendix $B$ and $C$ we ran our synthetic experiments using HMMs and MULTI-HMMs, to contrast our results in Chapter 4. We found that their performance was not satisfactory. Nevertheless, since HMMs are well adopted and used in many applications we wished to offer an extension of the traditional HMM with features inspired by our GBN model. In Chapter 6 we found that our GBN-HMMs were in fact more appropriate than the traditional HMMs (in terms of likelihood) when the data at hand included both regimes and variables predicting regime changes.

In our minds, one of the questions that remains unanswered is how we explain the changes among regimes. That is, we see that relationships change (i.e. edges are reversed, added and removed), yet we cannot offer an explanation to exactly why these changes happen. One of the strongest arguments for 
using probabilistic graphical models is that they allow us to convey complex relationships in a compact manner, however we face a problem that when these complex relationships change it is not easy to explain why. Chapters 4 and 5 deal with associational relationships among random variables, making no claim to be causal, which also may make the narrative more difficult. A very interesting line of research that we suggest is to look at explaining these changes systematically by using the concept of CSI, which we began investigating in Chapter 7. It could be possible to introduce new latent variables in the regime models identified which define CSIs that would explain why the specific changes occur.

\subsection{Causal scenarios and gated models}

Our departure to causality in Chapter 7 is a recent one. While the focus throughout this thesis has been on BNs, it is natural to investigate replacing the BNs with any type of model connected with gates. Thus we offered the more general concept of gated models, and specifically showed how they can be used to express CSIs using both BNs and ADMGs. The causal scenarios that we described in Chapter 7 clearly cannot be captured using a single graph, thus already at a descriptive level we can see the benefit of using gated models. However, in an effort to strengthen the argument we showed how certain causal effects that are not identifiable in some contexts may be so in others, and how gated models can capture this extra knowledge. We have yet to investigate gated models for causal inference further, thus we leave for future work the development of learning algorithms and formal definition of criteria for causal effect identification. 



\section{Bibliography}

[1] M. A. King, The end of alchemy: money, banking, and the future of the global economy. W. W. Norton \& Company, 2016.

[2] J. Pearl, Probabilistic reasoning in intelligent systems: networks of plausible inference. Morgan Kaufman Publishers, 1988.

[3] M. Bendtsen, "Regimes in baseball players' career data," Data Mining and Knowledge Discovery, 2017, accepted.

[4] M. Bendtsen and J. M. Peña, "Modelling regimes with Bayesian network mixtures," in Proceedings of the Thirtieth Annual Workshop of the Swedish Artificial Intelligence Society, pp. 20-29, 2017.

[5] J. M. Peña and M. Bendtsen, "Causal effect identification in acyclic directed mixed graphs and gated models," International Journal of Approximate Reasoning, 2017, accepted.

[6] M. Bendtsen, "Regime aware learning," in Proceedings of the Eighth International Conference on Probabilistic Graphical Models, pp. 112, 2016.

[7] M. Bendtsen and J. M. Peña, "Gated Bayesian networks for algorithmic trading," International Journal of Approximate Reasoning, vol. 69, no. 1, pp. 58-80, 2016.

[8] M. Bendtsen, "Bayesian optimisation of gated Bayesian networks for algorithmic trading," in Proceedings of the Twelfth Annual Bayesian Modeling Applications Workshop, pp. 2-11, 2015.

[9] M. Bendtsen and J. M. Peña, "Learning gated Bayesian networks for algorithmic trading," in Proceedings of the Seventh European Workshop on Probabilistic Graphical Models, pp. 49-64, 2014. 
[10] M. Bendtsen and J. M. Peña, "Gated Bayesian networks," in Proceedings of the Twelfth Scandinavian Conference on Artificial Intelligence, pp. 35-44, 2013.

[11] J. Pearl, Causality: models, reasoning and inference. Cambridge University Press, 2000.

[12] F. V. Jensen and T. D. Nielsen, Bayesian networks and decision graphs. Springer, 2007.

[13] K. B. Korb and A. E. Nicholson, Bayesian artificial intelligence. Taylor and Francis Group, 2011.

[14] D. Heckerman, "A tutorial on learning with Bayesian networks," Tech. Rep. MSR-TR-95-06, Microsoft Research, 1995.

[15] C. Yuan and B. Malone, "Learning optimal Bayesian networks: a shortest path perspective," Journal of Artificial Intelligence Research, vol. 48, no. 1, pp. 23-65, 2013.

[16] D. Sonntag, J. M. Peña, A. Hyttinen, and M. Järvisalo, "Learning optimal chain graphs with answer set programming," in Proceedings of the Thirty-First Conference on Uncertainty in Artificial Intelligence, pp. 822-831, 2015.

[17] J. Gama, I. Žliobaitè, A. Bifet, M. Pechenizkiy, and A. Bouchachia, "A survey on concept drift adaptation," ACM Computing Surveys, vol. 46, no. 4, pp. 1-37, 2014.

[18] C. Bielza and P. Larrañaga, "Discrete Bayesian network classifiers: a survey," ACM Computing Surveys, vol. 47, no. 3, pp. 1-43, 2014.

[19] F. V. Jensen and M. Vomlelová, "Unconstrained influence diagrams," in Proceedings of the Eighteenth Annual Conference on Uncertainty in Artificial Intelligence, pp. 234-241, 2002.

[20] F. V. Jensen, T. D. Nielsen, and P. P. Shenoy, "Sequential influence diagrams: a unified asymmetry framework," International Journal of Approximate Reasoning, vol. 42, no. 1-2, pp. 101-118, 2006.

[21] C. Boutilier, N. Friedman, M. Goldszmidt, and D. Koller, "Contextspecific independence in Bayesian networks," in Proceedings of the Twelfth Annual Conference on Uncertainty in Artificial Intelligence, pp. 115-123, 1996. 
[22] H. Nyman, J. Pensar, T. Koski, and J. Corander, "Stratified graphical models - context specific independence in graphical models," Bayesian Analysis, vol. 9, no. 4, pp. 883-908, 2014.

[23] J. E. Smith, S. Holtzman, and J. E. Matheson, "Structuring conditional relationships in influence diagrams," Operations Research, vol. 41, no. 2, pp. 280-297, 1993.

[24] D. Poole and N. L. Zhang, "Exploiting contextual independence in probabilistic inference," Journal of Artificial Intelligence Research, vol. 18, no. 1, pp. 263-313, 2003.

[25] A. Zagorecki and M. Druzdzel, "An empirical study of probability elicitation under noisy-OR assumption," in Proceedings of the Seventeenth International Florida Artificial Intelligence Research Society Conference, pp. 880-885, 2004.

[26] J. Q. Smith and P. E. Anderson, "Conditional independence and chain event graphs," Artificial Intelligence, vol. 172, no. 1, pp. 42-68, 2008.

[27] L. M. Barclay, R. A. Collazo, J. Q. Smith, P. A. Thwaites, and A. E. Nicholson, "The dynamic chain event graph," Electronic Journal of Statistics, vol. 9, no. 2, pp. 2130-2169, 2015.

[28] Y. Xiang and V. Lesser, "Justifying multiply sectioned Bayesian networks," in Proceedings of the Fourth International Conference on Multi-Agent Systems, pp. 349-356, 2000.

[29] Y. Xiang, "Multiply sectioned Bayesian networks and junction forests for large knowledge-based systems," Computational Intelligence, vol. 9, no. 2, pp. 171-220, 1993.

[30] S. Langevin, M. Valtorta, and M. Bloemeke, "Agent-encapsulated Bayesian networks and the rumor problem," in Proceedings of the Nineth International Conference on Autonomous Agents and Multiagent Systems, pp. 1553-1554, 2010.

[31] D. Koller and A. Pfeffer, "Object-oriented Bayesian networks," in Proceedings of the Thirteenth Annual Conference on Uncertainty in Artificial Intelligence, pp. 302-313, 1997.

[32] P. Treleaven, M. Galas, and V. Lalchand, "Algorithmic trading review," Communications of the ACM, vol. 56, no. 1, pp. 76-85, 2013. 
[33] G. Nuti, M. Mirghaemi, P. Treleaven, and C. Yingsaeree, "Algorithmic trading," Computer, vol. 44, no. 1, pp. 61-69, 2011.

[34] R. K. Narang, Inside the black box. John Wiley \& Sons, Inc., 2013.

[35] H. Markowitz, "Portfolio selection," The Journal of Finance, vol. 7, no. 1, pp. 77-91, 1952.

[36] R. Rebonato and A. Denev, Portfolio management under stress: a Bayesian-net approach to coherent asset allocation. Cambridge University Press, 2013.

[37] B. Li and C. H. Hoi, "Online portfolio selection: a survey," ACM Computing Surveys, vol. 46, no. 3, pp. 1-36, 2014.

[38] T. M. Cover, "Universal portfolios," Mathematical Finance, vol. 1, no. 1, pp. 1-29, 1991.

[39] D. P. Helmbold, R. E. Schapire, Y. Singer, and M. K. Warmuth, "Online portfolio selection using multiplicative updates," Mathematical Finance, vol. 8, no. 4, pp. 325-347, 1998.

[40] R. Pardo, The evaluation and optimization of trading strategies. John Wiley \& Sons, Inc., 2008.

[41] E. P. Chan, Quantitative trading. John Wiley \& Sons, Inc., 2009.

[42] W. F. Sharpe, "Likely gains from market timing," Financial Analysts Journal, vol. 31, no. 1, pp. 60-69, 1975.

[43] M. Webb-Somerset, "Shameful secrets of 'active' fund managers." Financial Times, 2014.

[44] R. Wigglesworth and S. Foley, "Active asset managers knocked by shift to passive strategies.” Financial Times, 2016.

[45] J. J. Murphy, Technical analysis of the financial markets. New York Institute of Finance, 1999.

[46] R. D. Edwards and J. Magee, Technical analysis of stock trends. Martino Publishing, 1957.

[47] S. A. Nelson, The ABC of stock speculation. Reprinted in 2012 by Forgotten Books, First published in 1903. 
[48] W. P. Hamilton, The stock market barometer. Reprinted in 2005 by Cosimo Classics, First published in 1932.

[49] R. Rhea, The Dow theory. Reprinted in 1994 by Frasier Publishing Co., First published in 1932.

[50] R. N. Elliot, The wave principle. Reprinted in 2012 by Snowball Publishing, First published in 1938.

[51] G. L. Morris, Candlestick charting explained: timeless techniques for trading stocks and futures. McGraw-Hill, 2006.

[52] W. J. J. Wilder, New concepts in technical trading systems. Hunter Publishing, 1978.

[53] "Journal of Technical Analysis." www.mta.org/development/journalof-technical-analysis/.

[54] “IFTA Journal.” www.ifta.org/publications/journal/.

[55] L. J. Tashman, "Out-of-sample tests of forecasting accuracy: an analysis and review," International Journal of Forecasting, vol. 11, no. 1, pp. 437-450, 2000.

[56] E. Brochu, V. M. Cora, and N. de Freitas, "A tutorial on Bayesian optimization of expensive cost functions, with application to active user modeling and hierarchical reinforcement learning," Tech. Rep. UBC TR-2009-023 and arXiv:1012.2599, 2009.

[57] C. E. Rasmussen and C. K. I. Williams, Gaussian processes for machine learning. MIT Press, 2006.

[58] Wikipedia, "Short (finance) - Wikipedia, the free encyclopedia," 2017. [Online; accessed 24-April-2017].

[59] M. J. Pring, Technical analysis explained. McGraw-Hill, 2002.

[60] M. Scheffer, S. Carpenter, J. A. Foley, C. Folke, and B. Walkerk, "Catastrophic shifts in ecosystems," Nature, vol. 413, no. 6856, pp. 591-596, 2001.

[61] S. Wooldridge, T. Done, R. Berkelmans, R. Jones, and P. Marshall, "Precursors for resilience in coral communities in a warming climate: a belief network approach," Marine Ecology-Progress Series, vol. 295, no. 1, pp. 157-169, 2005. 
[62] T. Andersen, J. Carstensen, E. Hernandez-Garcia, and C. M. Duarte, "Ecological thresholds and regime shifts: approaches to identification," Trends in Ecology \& Evolution, vol. 24, no. 1, pp. 49-57, 2009.

[63] S. M. Goldfeld and R. E. Quandt, "A Markov model for switching regressions," Journal of Econometrics, vol. 1, no. 1, pp. 3-16, 1973.

[64] J. D. Hamilton, "A new approach to the economic analysis of nonstationary time series and the business cycle," Econometrica, vol. 57, no. 2, pp. 357-384, 1989.

[65] G. R. Harris, "Markov chain Monte Carlo estimation of regime switching vector autoregressions," ASTIN bulletin, vol. 29, no. 1, pp. 47-79, 1999.

[66] J. Y. Campbell, A. W. Lo, and C. A. MacKinlay, The econometrics of financial markets. Princeton University Press, 1997.

[67] R. M. May, S. A. Levin, and G. Sugihara, "Complex systems: ecology for bankers,” Nature, vol. 451, no. 7181, pp. 893-895, 2008.

[68] M. Laurent and N. Kellershohn, "Multistability: a major means of differentiation and evolution in biological systems," Trends in biochemical sciences, vol. 24, no. 11, pp. 418-422, 1999.

[69] M. Pal, A. K. Pal, S. Ghosh, and I. Bose, "Early signatures of regime shifts in gene expression dynamics," Physical Biology, vol. 10, no. 3, p. $036010,2013$.

[70] A. Gelman, J. B. Carlin, H. S. Stern, and D. B. Rubin, Bayesian data analysis. Taylor \& Francis, 2003.

[71] S. Chib and E. Greenberg, "Understanding the metropolis-hastings algorithm," The American Statistician, vol. 49, no. 4, pp. 327-335, 1995.

[72] A. O. Munagi, "Set partitions with successions and separations," International Journal of Mathematics and Mathematical Sciences, vol. 2005, no. 3, pp. 451-463, 2005.

[73] W. Buntine, "Theory refinement on Bayesian networks," in Proceedings of the Seventh Conference on Uncertainty in Artificial Intelligence, pp. 52-60, 1991. 
[74] W. Lam and F. Bacchus, "Using new data to refine a Bayesian network," in Proceedings of the Tenth international Conference on Uncertainty in Artificial Intelligence, pp. 383-390, 1994.

[75] N. Friedman and M. Goldszmidt, "Sequential update of Bayesian network structure," in Proceedings of the Thirteenth Conference on Uncertainty in Artificial Intelligence, pp. 165-174, 1997.

[76] W. Lam, "Bayesian network refinement via machine learning approach," IEEE Transactions on Pattern Analysis and Machine Intelligence, vol. 20, no. 3, pp. 240-251, 1998.

[77] S. H. Nielsen and T. D. Nielsen, "Adapting Bayes network structures to non-stationary domains," International Journal of Approximate Reasoning, vol. 49, no. 2, pp. 379-397, 2008.

[78] J. W. Robinson and A. J. Hartemink, "Learning non-stationary dynamic Bayesian networks," Journal of Machine Learning Research, vol. 11, no. 1, pp. 3647-3680, 2010.

[79] Z. Ghahramani and G. E. Hinton, "Variational learning for switching state-space models," Neural Computation, vol. 12, no. 4, pp. 831-864, 2000 .

[80] F. Guo, S. Hanneke, W. Fu, and E. P. Xing, "Recovering temporally rewiring networks: a model-based approach," in Proceedings of the Twenty-Fourth International Conference on Machine Learning, pp. 321-328, 2007.

[81] X. Xuan and K. Murphy, "Modeling changing dependency structure in multivariate time series," in Proceedings of the Twenty-Fourth International Conference on Machine Learning, pp. 1055-1062, 2007.

[82] C. Chow and C. Liu, "Approximating discrete probability distributions with dependence trees," IEEE Transactions on Information Theory, vol. 14, no. 3, pp. 462-467, 1968.

[83] N. Silver, The signal and the noise. Penguin, 2012.

[84] M. Lewis, Moneyball. W.W. Norton \& Company, 2003.

[85] B. McTaggart, "Analyze this: Astros' Mejdal takes on unique role." www.mlb.com, 2012. 
[86] J. Langosch, "FBI concludes investigation on Astros breach." www.mlb.com, 2015.

[87] “Baseball prospectus." www.baseballprospectus.com.

[88] "Bill James online." www.billjamesonline.com.

[89] "Baseball think factory." www.baseballthinkfactory.org.

[90] “Steamer." www.steamerprojections.com.

[91] “Marcel." www.tangotiger.net/marcel.

[92] J. Albert, "Beyond runs expectancy," Journal of Sports Analytics, vol. 1, no. 1, pp. 3-18, 2015.

[93] C. L. Morey and M. A. Cohen, "Bias in the $\log 5$ estimation of outcome of batter/pitcher matchups, and an alternative," Journal of Sports Analytics, vol. 1, no. 1, pp. 65-76, 2015.

[94] J. Heumann, "An improvement to the baseball statistic "pythagorean wins"," Journal of Sports Analytics, vol. 2, no. 2, pp. 49-59, 2016.

[95] "Retrosheet." www.retrosheet.org.

[96] Financial Crisis Inquiry Commission, The financial crisis inquiry report: final report of the national commission on the causes of the financial and economic crisis in the United States. U.S. Government Printing Office, 2011.

[97] M. D. Flood, J. C. Liechty, and T. Piontek, "Systemwide commonalities in market liquidity," Tech. Rep. 15-11, OFR working paper series, 2015.

[98] T. F. Geithner, Stress test. Broadway Books, 2014.

[99] N. Friedman and I. Nachman, "Gaussian process networks," in Proceedings of the Sixteenth Conference on Uncertainty in Artificial Intelligence, pp. 211-219, 2000.

[100] K. P. Murphy, Machine learning: a probabilistic perspective. The MIT press, 2012. 
[101] S. Bengio and Y. Bengio, "An EM algorithm for asynchronous input/output hidden Markov models," in Proceedings of the International Conference On Neural Information Processing, pp. 328-334, 1996.

[102] Y. Li, "Hidden Markov models with states depending on observations," Pattern Recognition Letters, vol. 26, no. 7, pp. 977-984, 2005.

[103] J. A. Bilmes, "Dynamic Bayesian multinets," in Proceedings of the Sixteenth Conference on Uncertainty in Artificial Intelligence, pp. 3845, 2000.

[104] M. L. P. Bueno, A. Hommersom, P. J. F. Lucas, S. Verwer, and A. Linard, "Learning complex uncertain states changes via asymmetric hidden Markov models: an industrial case," in Proceedings of the Eighth International Conference on Probabilistic Graphical Models, pp. 50-61, 2016.

[105] C. M. Bishop, Pattern recognition and machine learning. Springer, 2013.

[106] N. Friedman, "The Bayesian structural EM algorithm," in Proceedings of the Fourteenth Conference on Uncertainty in Artificial Intelligence, pp. 129-138, 1998.

[107] G. Melançon, I. Dutour, and M. Bousquet-Mélou, "Random generation of directed acyclic graphs," Electronic Notes in Discrete Mathematics, vol. 10, no. 1, pp. 202-207, 2001.

[108] J. S. Ide and F. G. Cozman, "Random generation of Bayesian networks," in Brazilian Symposium on Artificial Intelligence, pp. 366376, 2002. 



\section{Appendix A \\ Pseudocode for the regime detection learning algorithm}

In this appendix we shall present pseudocode for the learning algorithm introduced and discussed in Chapter 4 . As we did in Section 4.2, we will divide the algorithm into three distinct parts: identifying regime changes, learning the transition structure, and creating a GBN.

\section{A.1 Identifying regime changes in the data set}

The pseudocode for the initial identification of regime transitions can be found in Figure A.1. The function IDENTIFY is called using a data set $\mathcal{D}$, the maximum number of transitions $k$, and the number of MCMC iterations as input. The algorithm begins by initialising the current parameters. To distinguish between current and proposed parameters, we use a superscript $c$ for the current parameters, and superscript $p$ for the proposed parameters.

On line 9 the MCMC iterations begin. Proposed parameters are sampled according to their proposal distributions that are described in detail in Section 4.2.1. Two sets of nonzero $\delta$ s are calculated, one for the current parameters $\Gamma^{c}$ and one for the proposed parameters $\Gamma^{p}$. On line 18 , the data set supplied to the algorithm is split according to the nonzero $\delta$ s (again, this is done for both the current and the proposed parameters). For each resulting subset of $\mathcal{D}$ a BN is learnt, resulting in a set of BNs for the current parameters, and a set of BNs for the proposed parameters.

On lines 23 through 26 we calculate the likelihood of the data given the current $\mathrm{BNs}$ and the proposed BNs, as well as the posterior probability of the current and proposed parameters. Note that the calculations are described in more detail in Equations 4.1 and 4.5 . 
1: function $\operatorname{IDENTIFY}(\mathcal{D}, k$, iterations $)$

2: $\quad n \leftarrow$ number of data points in $\mathcal{D}$

3: $\quad \beta_{1}^{c}=\frac{n}{k+1}, \beta_{2}^{c}=\frac{2 n}{k+1}, \ldots, \beta_{k}^{c}=\frac{k n}{k+1}$

4: $\quad I_{1}^{c}=1, \ldots, I_{k}^{c}=1$

5: $\quad \delta_{1}^{c}=\beta_{1}^{c} I_{1}^{c}, \ldots, \delta_{k}^{c}=\beta_{k}^{c} I_{k}^{c}$

6: $\quad$ iteration $=0$

7: $\quad \mathbf{I} \leftarrow\{\}$

8: $\Delta \leftarrow\{\}$

9: repeat

10: $\quad$ iteration $=$ iteration +1

11: $\delta_{1}^{p}=\beta_{1}^{p} I_{1}^{p}, \ldots, \delta_{k}^{p}=\beta_{k}^{p} I_{k}^{p}$

$$
r=\frac{p\left(\beta_{1}^{p}, \ldots, \beta_{k}^{p}, I_{1}^{p}, \ldots, I_{k}^{p} \mid \mathcal{D}\right) / J^{p}}{p\left(\beta_{1}^{c}, \ldots, \beta_{k^{\prime}}^{c} I_{1}^{c}, \ldots, I_{k}^{c} \mid \mathcal{D}\right) / J^{c}}
$$$$
u=U(0,1)
$$

if $\mathrm{r}>\mathrm{u}$ then

if iteration $>$ iterations $/ 2$ then

$$
\begin{aligned}
& \beta_{1}^{c}=\beta_{1}^{p}, \ldots, \beta_{k}^{c}=\beta_{k}^{p} \\
& I_{1}^{c}=I_{1}^{p}, \ldots, I_{k}^{c}=I_{k}^{p} \\
& \delta_{1}^{c}=\beta_{1}^{c} I_{1}^{c}, \ldots, \delta_{k}^{c}=\beta_{k}^{c} I_{k}^{c}
\end{aligned}
$$

end if

$$
\text { end if }
$$

$$
\begin{gathered}
\mathbf{I}=\mathbf{I} \cup\left\{I_{1}^{c}, \ldots, I_{k}^{c}\right\} \\
\Delta=\Delta \cup\left\{\delta_{1}^{c}, \ldots, \delta_{k}^{c}\right\}
\end{gathered}
$$

$$
\text { until iteration }==\text { iterations }
$$

return nonzero $\delta$ s.

end function

iteration $=$ iteration +1
$\beta_{1}^{p}, \ldots, \beta_{k}^{p} \leftarrow$ propose new $\beta$ s according to Equations 4.6 through 4.10

$I_{1}^{p}=\operatorname{Bernoulli}(0.5), \ldots, I_{k}^{p}=\operatorname{Bernoulli}(0.5) \quad \triangleright$ Propose new indicator values

$$
\begin{array}{lr}
\Gamma^{c}=\left\{\delta_{i} \in\left\{\delta_{1}^{c}, \ldots, \delta_{k}^{c}\right\}: \delta_{i} \neq 0\right\} & \triangleright \text { Select current nonzero } \delta s \\
\Gamma^{p}=\left\{\delta_{i} \in\left\{\delta_{1}^{p}, \ldots, \delta_{k}^{p}\right\}: \delta_{i} \neq 0\right\} & \triangleright \text { Select proposed nonzero } \delta s \\
\mathbf{D}^{c} \leftarrow \text { split } \mathcal{D} \text { into subsets according to } \Gamma^{c} & \\
\mathbf{B N}^{c}=\left\{D_{i}^{c} \in \mathbf{D}^{c}: \text { Learn a BN using } D_{i}^{c}\right\} & \triangleright \text { Learn a BN for each subset } \\
\mathbf{D}^{p} \leftarrow \text { split } \mathcal{D} \text { into subsets according to } \Gamma^{p} & \\
\mathbf{B N}^{p}=\left\{D_{i}^{p} \in \mathbf{D}^{p}: \text { Learn a BN using } D_{i}^{p}\right\} & \triangleright \text { Learn a BN for each subset }
\end{array}
$$

$\triangleright$ Space out $\beta$ s evenly $\triangleright$ Initially set all splits to 1

$\triangleright$ Container for posterior samples $\triangleright$ Container for posterior samples

$$
\begin{aligned}
& p\left(\mathcal{D} \mid \Gamma^{c}\right)=p\left(D_{1}^{c} \mid B N_{1}^{c}\right) \ldots p\left(D_{k^{\prime}}^{c} \mid B N_{k^{\prime}}^{c}\right) \quad \triangleright k^{\prime} \text { is the number of elements in } \Gamma^{c} \\
& p\left(\beta_{1}^{c}, \ldots, \beta_{k}^{c}, I_{1}^{c}, \ldots, I_{k}^{c} \mid \mathcal{D}\right)=p\left(\mathcal{D} \mid \Gamma^{c}\right) U\left(\beta_{1}^{c} ; 0, \beta_{2}^{c}\right) \ldots U\left(\beta_{k^{c}}^{c} \beta_{k-1}^{c}, n+1\right) p\left(I_{1}^{c}\right) \ldots p\left(I_{k}^{c}\right) \\
& p\left(\mathcal{D} \mid \Gamma^{p}\right)=p\left(D_{1}^{p} \mid B N_{1}^{p}\right) \ldots p\left(D_{k^{\prime}}^{p} \mid B N_{k^{\prime}}^{p}\right) \quad \square k^{\prime} \text { is the number of elements in } \Gamma^{p} \\
& p\left(\beta_{1}^{p}, \ldots, \beta_{k^{\prime}}^{p}, I_{1}^{p}, \ldots, I_{k}^{p} \mid \mathcal{D}\right)=p\left(\mathcal{D} \mid \Gamma^{p}\right) U\left(\beta_{1}^{p} ; 0, \beta_{2}^{p}\right) \ldots U\left(\beta_{k}^{p} ; \beta_{k-1}^{p}, n+1\right) p\left(I_{1}^{p}\right) \ldots p\left(I_{k}^{p}\right)
\end{aligned}
$$

$J^{p}=p\left(\beta_{1}^{p}, \ldots, \beta_{k}^{p} \mid \beta_{1}^{c}, \ldots, \beta_{k}^{c}\right) p\left(I_{1}^{p}, \ldots, I_{k}^{p} \mid I_{1}^{c}, \ldots, I_{k}^{c}\right) \quad \triangleright$ Probability going from $c$ to $p$ $J^{c}=p\left(\beta_{1}^{c}, \ldots, \beta_{k}^{c} \mid \beta_{1}^{p}, \ldots, \beta_{k}^{p}\right) p\left(I_{1}^{c}, \ldots, I_{k}^{c} \mid I_{1}^{p}, \ldots, I_{k}^{p}\right) \quad \triangleright$ Probability going from $p$ to $c$

nonzero $\delta s=\left\{i \in 1, \ldots, k: \operatorname{Mean}_{\Delta}\left(\delta_{k}\right)\right.$ if $\left.\operatorname{Mean}_{\mathbf{I}}\left(I_{k}\right)>0.5\right\}$

Figure A.1: Pseudocode for identification of nonzero $\delta s$.

The rest of the algorithm decides if the current parameters should be replaced with the proposed parameters. First calculate the probability of moving from the current parameters to the proposed (and vice versa). Then calculate the acceptance ratio $r$ and accept the proposed parameters with this 
probability (if $r>1$ then always accept). If the proposed parameters are accepted, then we set the current parameters to the proposed parameters.

Since we use a burn-in period to throw away samples at the beginning of the MCMC iterations, on line 39 we only store posterior samples if the current iteration is more than half the total iterations (i.e. throw away first half of samples).

Once all MCMC iterations are complete, we identify which $\delta$ s are nonzero on line 45. Given the posterior samples $\mathbf{I}$, we calculate the marginal mean for each indicator $I$, and if the mean is greater than 0.5 , then the corresponding $\delta$ is considered nonzero. The value for the $\delta$ parameter is the marginal mean given the posterior samples $\Delta$.

Finally, we return the nonzero $\delta$ s identified in the data set.

\section{A.2 Merging subsets}

The pseudocode for the transition structure identification part of the overall learning algorithm is presented in Figure A.2. The function MERGE takes as input a data set $\mathcal{D}$ and the nonzero $\delta$ s identified by the IDENTIFY function described in Section 4.2.1 and Section A.1

The first three operations first split the supplied data set into subsets, according to the supplied nonzero $\delta \mathrm{s}$. Then two mappings are created that together define a regime transition structure. First, $\mathbf{R}$ is a mapping between regime labels, e.g. $R_{1}$ and $R_{2}$, to a subset of $\mathbf{D}$. That is, it defines which subsets of $\mathcal{D}$ belongs to which regimes. The second mapping, $\mathbf{C}$, defines the children of each regime, e.g. $C_{1}=\{2\}$ means that regime 2 is a child of regime 1 . If $C_{1}=\{2,3\}$ this would mean that both regime 2 and 3 are children of regime 1 . A regime transition structure can therefore be defined by a pair $\mathbf{R}$ and $\mathbf{C}$.

Since we wish to test all possible regime transition structures, we create a container $\mathcal{R C}$ to hold them, before we call the COLLAPSE function on line 7. Before discussing the COLLAPSE function, we note that the $M E R G E$ function ends by computing the marginal likelihood of the data $\mathcal{D}$ for each pair $\left\{\mathbf{R}_{k}, \mathbf{C}_{k}\right\}$ in $\mathcal{R C}$ and returns the pair (i.e. the transition structure) that has the highest marginal likelihood. Computing the marginal likelihood can be done using a simple restructuring of Equation 4.5 .

Inside the COLLAPSE function we create a nested loop to identify nonadjacent regimes. If two regimes are adjacent then we do not want to merge them (see the discussion in Section 4.2.2). Therefore, on line 16, we ignore cases where either regime $j$ is a child of regime $i$, or regime $i$ is a 


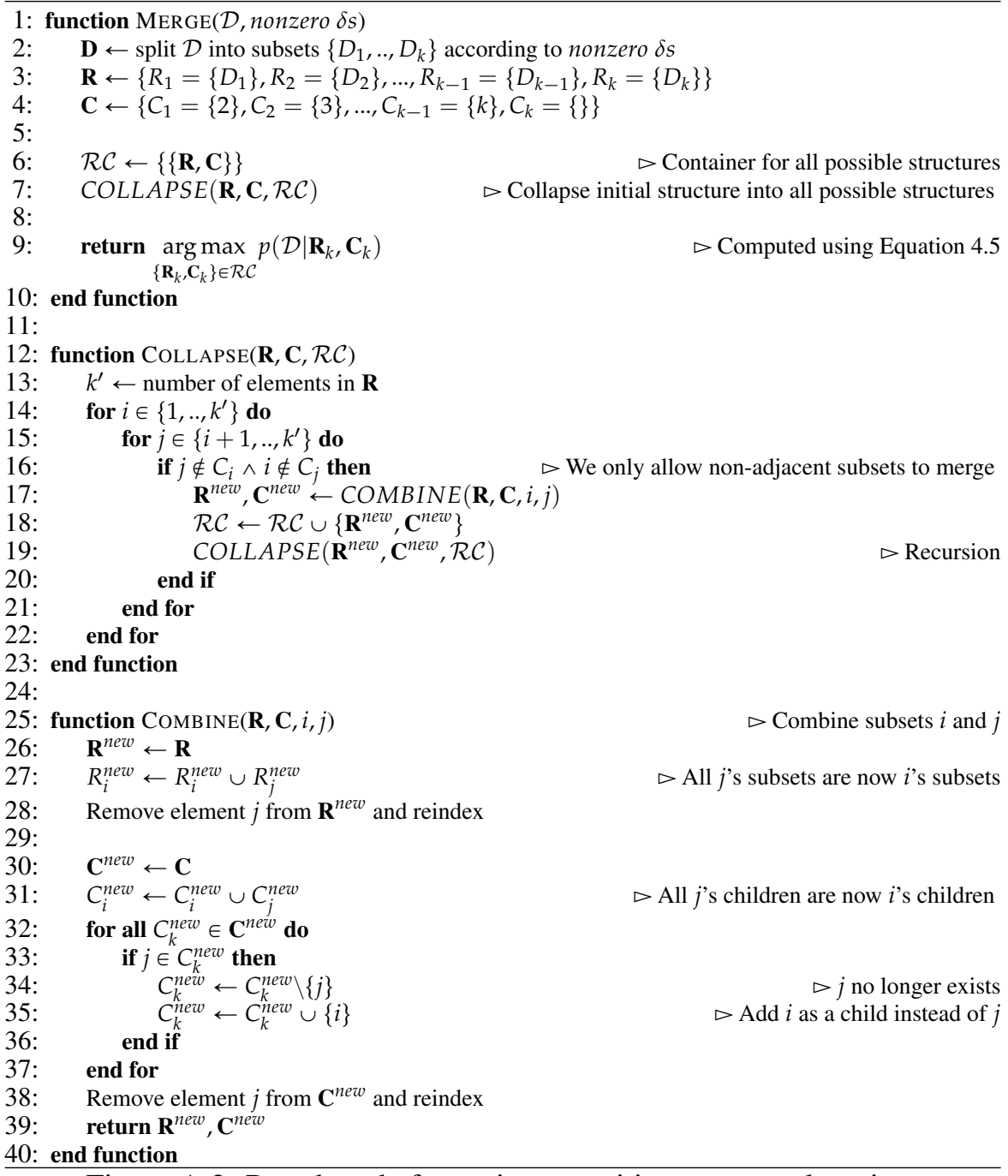

Figure A.2: Pseudocode for regime transition structure learning.

child of regime $j$. If two regimes are non-adjacent then we call the function COMBINE. This will result in a new regime transition structure (i.e. a new pair $\mathbf{R}^{\text {new }}, \mathbf{C}^{\text {new }}$ ), which we store in $\mathcal{R C}$ and use as input to COLLAPSE, thus creating a recursion. Once the recursion is complete, $\mathcal{R C}$ will contain all regime transition structures.

In COMBINE, the goal is to take a regime transition structure and create a new one by merging two non-adjacent regimes. The algorithm starts by copying $\mathbf{R}$ into $\mathbf{R}^{\text {new }}$ and then adding all data subsets that used to belong to regime $j$ to regime $i$ in $\mathbf{R}^{\text {new }}$ (thus we have merged the subsets). We then 
remove element $j$ from $\mathbf{R}^{\text {new }}$ and reindex the mapping (that is, any index that was higher than $j$ is reduced by one so as to avoid gaps in the regime labelling). For the mapping of child regimes, we must take a bit more caution. First, on line 31, children of regime $j$ are now children of regime $i$. However, all regimes that used to have $j$ as a child should now have $i$ as a child instead. The loop therefore goes through all child mappings and removes $j$ if it exists and adds $i$. Once the loop terminates, we remove element $j$ from $\mathbf{C}^{\text {new }}$ and reindex (again, to avoid gaps in the regime labels). Finally, we return the new regime transition structure.

\section{A.3 Constructing a gated Bayesian network}

The final step of the learning algorithm is to construct a GBN. In Figure A.3 we present the pseudocode for this part of the algorithm. As was the case in Section A.2, $\mathbf{R}$ maps each regime to subsets of $\mathcal{D}$. The algorithm begins by learning a $\mathrm{BN}$ for each regime, and connects these BNs using gates according to the child relationships contained in $\mathbf{C}$. Thereafter, initial values for the parameters of the gates $(\tau$ and $\theta)$ are chosen. The number of resamples, iterations and the exploration/exploitation coefficient $\eta$ is set.

To reduce the risk of overfitting the test data, on line 10 we resample $\mathcal{D}$ while preserving the regimes according to $\mathbf{R}$. This is described in more detail in Section 4.2.3, however the idea is that we take the subsets that belong to the first regime and resample these, placing them in the new sample such that the regime transition structure is intact, and then continue to the second regime, and so on. We then create our proxy random variable $g$ (that follows a GP) and containers to store parameters and function values (i.e. the accuracies).

On line 16 the main Bayesian optimisation loop begins. We calculate the average accuracy of the GBN parameterised with $\tau$ and $\theta$ over the resamples. We store the parameters and the accuracy, and then identify the next pair $\tau$ and $\theta$ by maximising the UCB (upper confidence bound criterion discussed in Section 3.4.2). Once a predefined number of iterations of Bayesian optimisation has completed, we parameterise the GBN with the parameters that gave the maximum accuracy, and return the parameterised GBN (thus ending the learning algorithm).

In the ACCURACY function we calculate the accuracy of a pair of parameters $\theta$ and $\tau$ over all resamples. The steps taken are those described in Section 4.2.3. Essentially, we treat the resample as a stream of data, increasing its size with each iteration, and process it in the GBN. We compare which $\mathrm{BN}$ is active in the GBN to the true regime according to $\mathbf{R}$, and give a score 


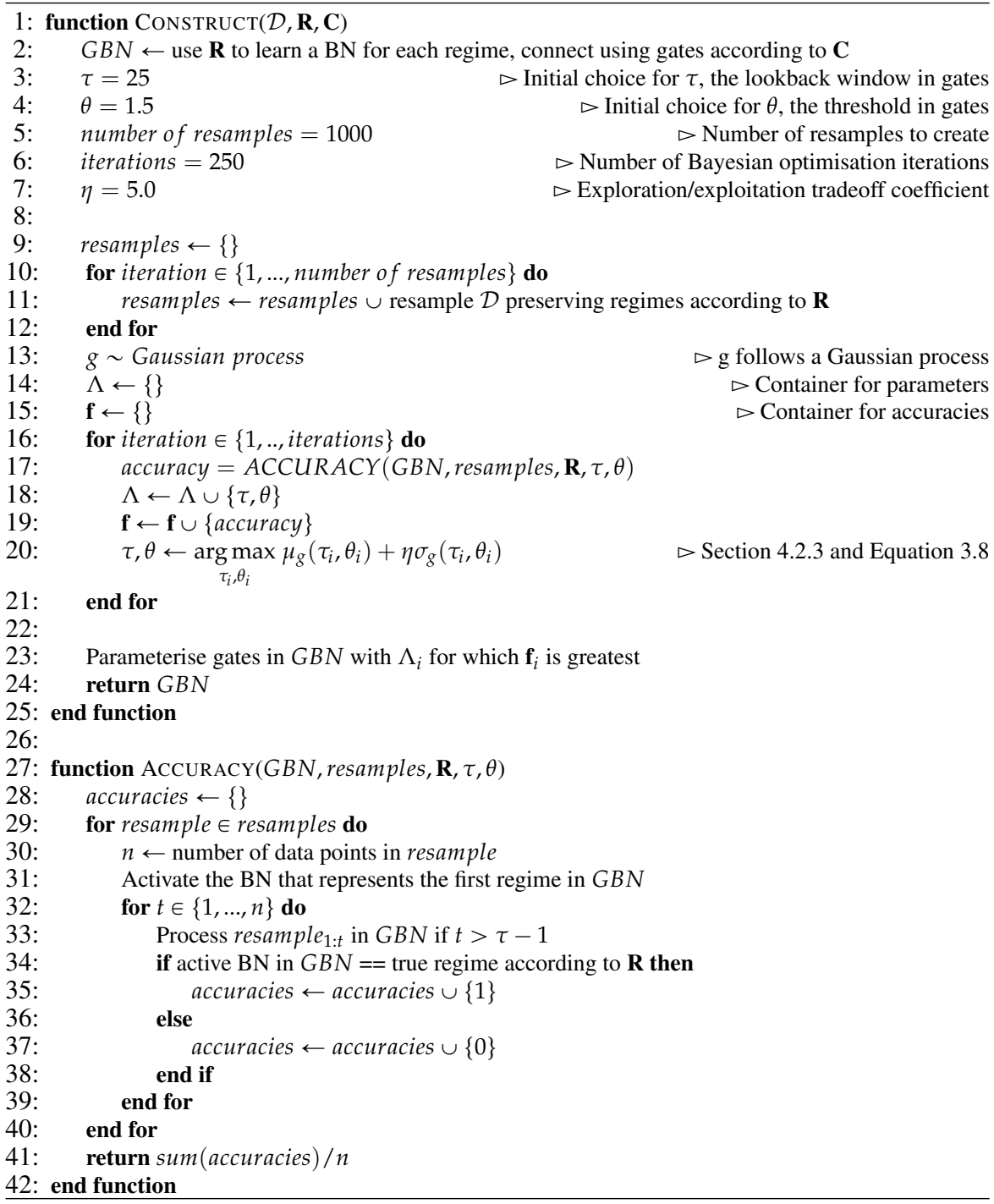

Figure A.3: Pseudocode for construction of a GBN.

of one if they match. To clarify, if data point with index 250 belongs to a subset of $\mathcal{D}$ that in turn belongs to regime $R_{3}$ (which we can read off from $\mathbf{R})$, then if the $\mathrm{BN}$ that represents $R_{3}$ is active after the stream that ends with index 250 has been processed, then we say that the GBN is correct, all other cases are incorrect. Note that in order to calculate the ratios inside the gates we must have at least $\tau$ data points, therefore no processing of data is done until we reach this number. 


\section{Appendix B \\ Detecting regimes using hidden Markov models - experiments on synthetic data}

In Section 4.4 we discussed a set of experiments where regimes were identified in synthetic data sets. In this appendix we shall consider the same data, however this time using HMMs to identify the regime changes. We shall use two variants of the HMM:

- The traditional HMM, henceforth only referred to as HMM, which utilises a hidden variable to represent the state of the world and observable variables that are independent of each other given the hidden state.

- A variation on the traditional HMM where there is structure among the observable variables, such that for each state of the hidden variable an independence model is learnt among the observable variables.

The second variant is discussed in detail in Chapter 6 , and following the convention of that chapter we shall refer to it as MULTI-HMM (referencing the likeness with the Bayesian multinet approach). We shall not delve deeper into the details of how the parameters of these models are estimated, nor how the structure of the independence models are learnt, as both aspects are covered extensively in Chapter 6 and Appendix D

We recall that the aim is to show that, given a data set for a system that exhibits regimes, we can learn a model that captures the system's regimes and transition structure. 


\section{B.1 Methodology}

In Section 4.4 we considered data sets with zero to four splits, thus the system that generated the data had at minimum one regime and at maximum five. We included the case with zero splits to show that the GBNs that we learnt were able to detect the number of regimes in the data, even when there was only one. However we gave the models that we learnt in the current experiments a strong advantage over the GBNs: we fixed the number of regimes to the correct number of regimes prior to learning, and therefore the case with zero splits is irrelevant in the current setting and will not be reported.

We will continue to use the notation used in Section 4.4, where each of the data sets is a data set for a system, distinguishing between them using the notation $\mathcal{S}_{\text {split,hypothesis }}$, e.g. $\mathcal{S}_{3,1}$ is the first hypothesis with three splits and $\mathcal{S}_{4,5}$ is the fifth hypothesis with four splits. Please see Table 4.1 for all systems considered (except for $\mathcal{S}_{0,1}$ as previously discussed).

We estimated the parameters, and learnt the structure over the observable variables when applicable, for all of the 23 training samples. Again, please find details for the procedure for doing so in Chapter 6 and Appendix $D$. We then computed the posterior distribution over the hidden states for each data point in the data set using all the data, i.e. $p\left(H_{t} \mid \mathbf{o}_{1: T}\right)$. We considered the state which had the maximum posterior probability to be the regime identified by the model.

In Section 4.4 we reported the identified splits in the data and the distance from these splits to the true splits in the training data. However, as will become evident, this approach is not appropriate for the current experiments. Instead, we will report a plot for each model and system under consideration which shows the true regimes in the data, and the identified regimes by the two models.

\section{B.2 Results and discussion}

In Figure B.1 we present the results using the standard HMM. The figure should be interpreted as follows. The top left graph represents the experiments on system $S_{1,1}$. The x-axis represents data points and the y-axis represents the regime. The thick black line represents the true regime for each data point, i.e. data point with index 200 belongs to $R_{1}$, and data point with index 500 belongs to $R_{2}$. The thinner grey line represents the state with the maximum posterior probability according to the HMM. As is evident from the top left graph, the HMM cannot distinguish between the two regimes very well, but rather oscillates violently between $R_{1}$ and $R_{2}$. Also, the true regime 
transition structure is $R_{1} \rightarrow R_{2}$, however the HMM assigns a nonzero probability to the event that the system might go from $R_{2}$ to $R_{1}$, thus the regime transition structure that the HMM identifies is $R_{1} \leftrightarrows R_{2}$, which is incorrect.

At a glance, we can tell that the HMM does not perform very well with respect to identifying the correct regime and transition structure. It does well on system $S_{3,5}$, but for all others there is oscillation during at least one of the regimes.

While we gave the HMMs an advantage by inputting the correct number of regimes before learning the parameters (an advantage not given to our GBNs in our main experiments in Section 4.4, we must also point out that the odds are somewhat stacked against the HMMs due to two fundamental features of HMMs. First, a HMM does not learn a new independence model for each hidden state, thus actually capturing the differences among the regimes must be done in the parameters of the marginal and conditional distributions. Second, the HMM is a mixture model and it is therefore supposed to mix all regimes, weighted by the probability of each regime. The second feature is a distinguishing feature of HMMs, as the state is always hidden. However, the first feature we can change, allowing for a different independence model for each hidden state. The results for running the same experiments, but this time using the MULTI-HMM, are presented in Figure B.2.

From Figure B.2 we can see that the MULTI-HMM does better on systems $S_{1,1}, S_{2,1}$ and $S_{4,14}$, but that in general the results are similar as for the traditional HMM. Again, these models are mixture models, and they cannot discriminate among the distinct independence models that are present in the data. 

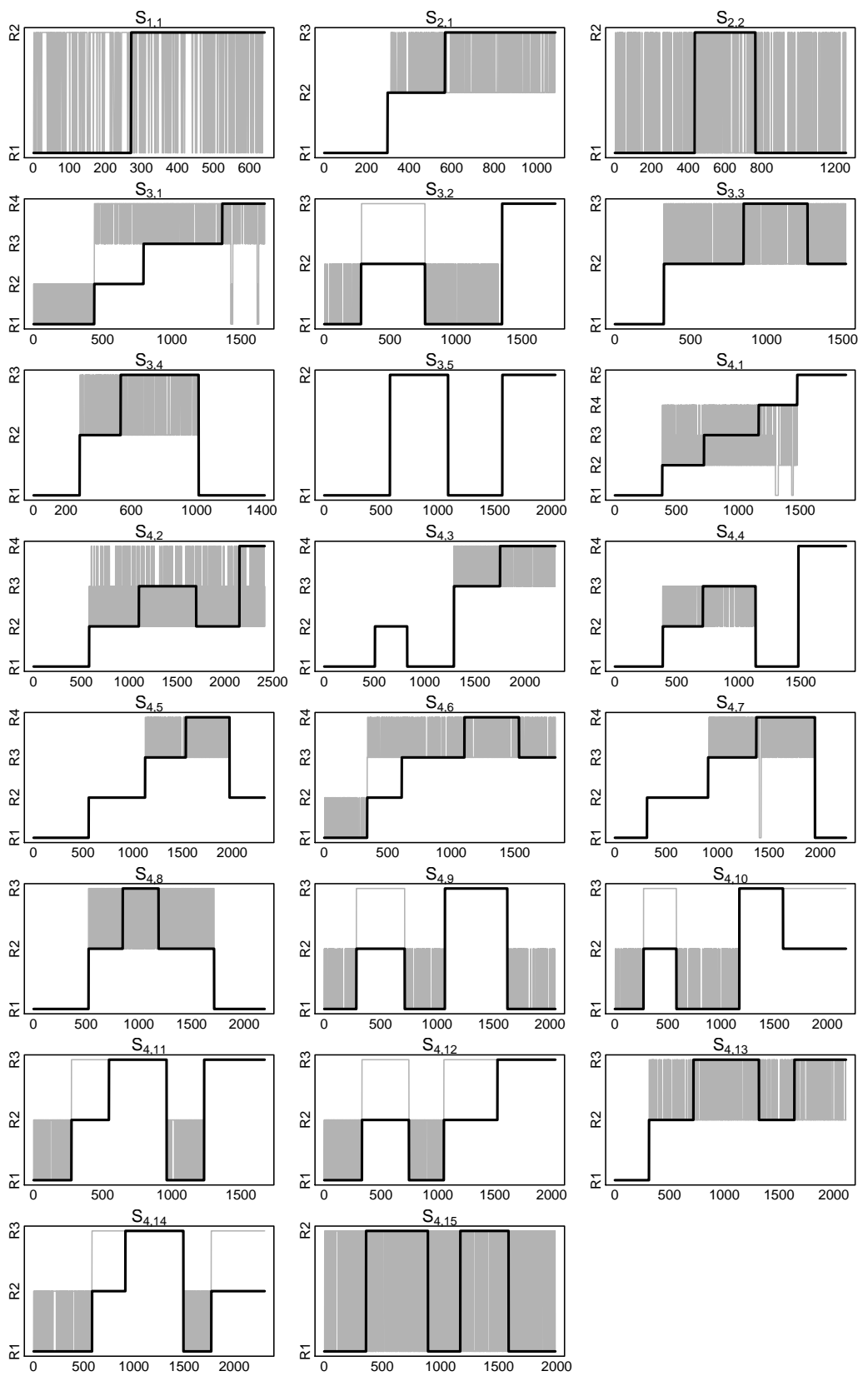

Figure B.1: True regimes (black line) versus the maximum probability state given a trained HMM (grey line). The $\mathrm{x}$-axis represents data points and the $\mathrm{y}$-axis the regime. 

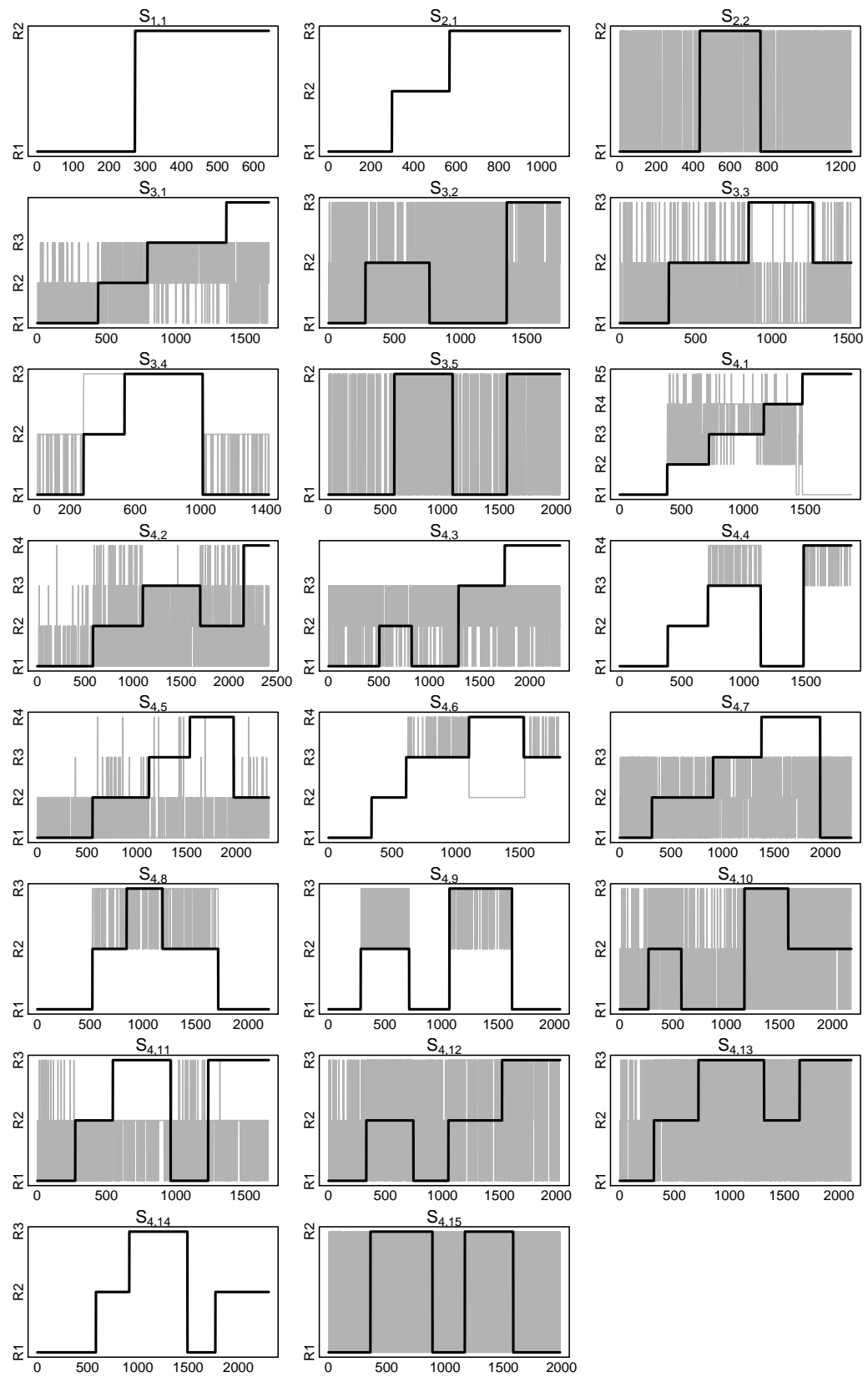

Figure B.2: True regimes (black line) versus the maximum probability state given a trained MULTI-HMM (grey line). The x-axis represents data points and the $y$-axis the regime. 



\section{Appendix C \\ Detecting regimes using hidden Markov models - experiments on synthetic baseball data}

In Section 4.5 .2 we introduced an experiment where regimes were identified in synthetic baseball data. As was the case in Appendix B, we shall in this appendix use HMMs to identify the regimes rather than GBNs. The difference between the experiments described here and those in Appendix $B$ is an increase in the number of random variables, and that some of the variables can take an increased number of states (described in Section 4.5.1). As before, we shall use two variants of the HMM:

- The traditional HMM, henceforth only referred to as HMM, which utilises a hidden variable to represent the state of the world and observable variables that are independent of each other given the hidden state.

- A variation on the traditional HMM where there is structure among the observable variables, such that for each state of the hidden variable an independence model is learnt among the observable variables. This variation is discussed in detail in Chapter 6 , and following the convention of that chapter we shall refer to it as MULTI-HMM.

We recall that the aim is to show that, given a data set for a system that exhibits regimes, we can learn a model that captures the system's regimes and transition structure. 


\section{C.1 Methodology}

In Section 4.5.2 we considered data sets generated from three different transition structures (depicted in Figure 4.7), which contained up to five different regimes (depicted in Figure 4.6. As can be seen in Figure 4.7 we included one case of non-reoccuring regimes and two cases where regimes reoccur.

We estimated the parameters, and learnt the structure over the observable variables when applicable, using the training samples for the three structures. We gave the HMMs a strong advantage over the GBNs that we learnt: we fixed the number of regimes to the correct number of regimes prior to learning. We then computed the posterior distribution over the hidden states for each data point in the data set using all the data, i.e. $p\left(H_{t} \mid \mathbf{o}_{1: T}\right)$. We considered the state which had the maximum posterior probability to be the regime identified by the model. We will report a plot for each model and system under consideration which shows the true regimes in the data, and the identified regimes by the two models.

\section{C.2 Results and discussion}

In Figure C.1 we present the results using the standard HMM. The top plot represents the experiments on Structure 1 (see Figure 4.7). The x-axis represents data points and the y-axis represents the regime. The thick black line represents the true regime for each data point, and the thinner grey line represents the state with the maximum posterior probability according to the HMM. As is evident from the top graph, the HMM cannot distinguish between regimes $R_{3}$ and $R_{4}$, but rather oscillates violently between the two. Also, the true regime transition structure is $R_{1} \rightarrow R_{2} \rightarrow R_{3} \rightarrow R_{4} \rightarrow R_{5}$, however the HMM shows that the system might go from $R_{4}$ to $R_{3}$, thus the regime transition structure that the HMM identifies is not the one that generated the data.

A similar pattern emerges when we look at the second and third plot in Figure C.1 (representing Structure 2 and Structure 3). The HMM cannot distinguish between $R_{1}$ and $R_{2}$ for Structure 2 (which we can see in the second plot), and does very poorly for Structure 3 , where it keeps oscillating between regimes throughout the entire data set.

The MULTI-HMM does particularly well for Structure 3, as can be seen in the last plot of Figure C.2 (although we still have to take into consideration that the HMMs were a priori given the number of regimes in the data). However, for Structure 1 and Structure 2 the results are not as promising, borderline disastrous for Structure 2. 

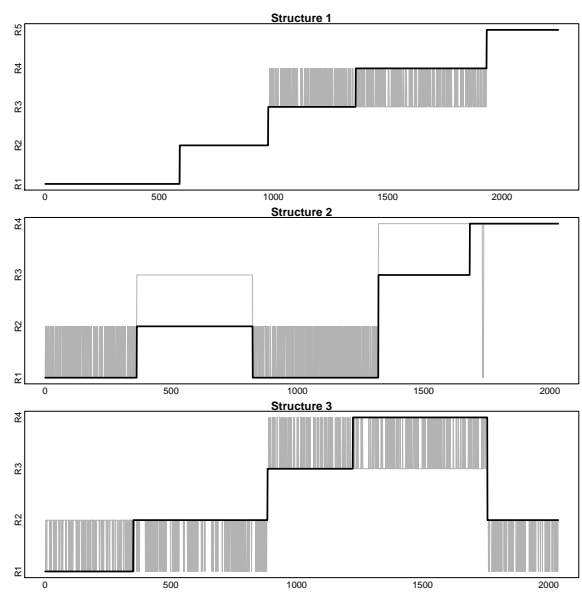

Figure C.1: True regimes (black line) versus the maximum probability state given a trained HMM (grey line). The $\mathrm{x}$-axis represents data points and the $y$-axis the regime.
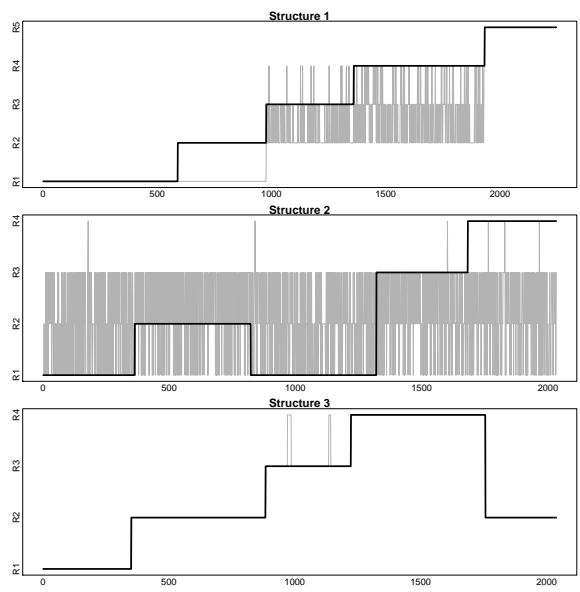

Figure C.2: True regimes (black line) versus the maximum probability state given a trained MULTI-HMM (grey line). The x-axis represents data points and the $y$-axis the regime. 



\section{Appendix D \\ Parameter estimation and inference in Bayesian network mixtures}

\section{D.1 Parameter estimation}

Since we cannot observe the hidden variables $H_{1: T}$, we cannot solve the parameter estimation problem by simply counting events from a data set. Instead we must apply some method that can give us an approximate solution. The canonical way of parameter estimation in HMM is to use EM, and therefore we shall also adopt this technique for our GBN-HMMs.

As before, let $\mathbf{0}_{1: T}$ represent a sequence of observations over the variables $\mathbf{O}_{1: T}$ and let $h_{1: T}=\left\{h_{1}, h_{2}, \ldots, h_{T}\right\}$ represent a sequence of states. Let $\mathcal{H}$ represent the set of all state sequences $h_{1: T}$. The current parameters for our model are denoted $\Theta^{\prime}$, and we seek parameters $\Theta$ that maximises the expected log-likelihood of the observed data. This expectation is maximised when $Q\left(\Theta, \Theta^{\prime}\right)=\sum_{h_{1: T} \in \mathcal{H}} p\left(\mathbf{o}_{1: T}, h_{1: T} \mid \Theta^{\prime}\right) \log p\left(\mathbf{o}_{1: T}, h_{1: T} \mid \Theta\right)$ is maximised.

We can substitute $p\left(\mathbf{o}_{1: T}, h_{1: T} \mid \Theta\right)$ in the $Q$ function with our factorisation of the GBN-HMM, which gives us the expanded $Q$ function in Equation D.1 
From this expansion we can see that the terms do not interact, thus we can maximise each term separately.

$$
\begin{aligned}
& Q\left(\Theta, \Theta^{\prime}\right)= \\
& \sum_{h_{1: T} \in \mathcal{H}} p\left(\mathbf{o}_{1: T}, h_{1: T} \mid \Theta^{\prime}\right) \log p\left(\mathbf{o}_{1: T}, h_{1: T} \mid \Theta\right)= \\
& \sum_{h_{1: T} \in \mathcal{H}} p\left(\mathbf{o}_{1: T}, h_{1: T} \mid \Theta^{\prime}\right) \log \pi_{h_{1}} \prod_{i=1}^{M} b_{h_{1}}^{i}\left(\mathbf{o}_{1}\right) \times \\
& \prod_{t=2}^{T} a_{h_{t-1}, h_{t}, z_{t-1}} \prod_{t=2}^{T} \prod_{i=1}^{M} b_{h_{t}}^{i}\left(\mathbf{o}_{t}\right)= \\
& \sum_{h_{1: T} \in \mathcal{H}} p\left(\mathbf{o}_{1: T}, h_{1: T} \mid \Theta^{\prime}\right) \log \pi_{h_{1}}+ \\
& \sum_{h_{1: T} \in \mathcal{H}} p\left(\mathbf{o}_{1: T}, h_{1: T} \mid \Theta^{\prime}\right) \sum_{t=2}^{T} \log a_{h_{t-1}, h_{t}, z_{t-1}}+ \\
& \sum_{h_{1: T} \in \mathcal{H}} p\left(\mathbf{o}_{1: T}, h_{1: T} \mid \Theta^{\prime}\right) \sum_{t=1}^{T} \sum_{i=1}^{M} \log b_{h_{t}}^{i}\left(\mathbf{o}_{t}\right)
\end{aligned}
$$

\section{D.1.1 Initial state distribution}

The first term in Equation D.1 can be seen as marginalising out all hidden state variables except the first. To show this we can look at a small example were $\mathcal{H}=\{\{1,1\},\{1,2\},\{2,1\},\{2,2\}\}$. If we expand the sum over these sequences we get Equation D.2.

$$
\begin{aligned}
& \sum_{h_{1: 2} \in \mathcal{H}} p\left(\mathbf{o}_{1: 2}, h_{1: 2} \mid \Theta^{\prime}\right) \log \pi_{h_{1}}= \\
& p\left(\mathbf{o}_{1: 2}, h_{1}=1, h_{2}=1 \mid \Theta^{\prime}\right) \log \pi_{1}+ \\
& p\left(\mathbf{o}_{1: 2}, h_{1}=1, h_{2}=2 \mid \Theta^{\prime}\right) \log \pi_{1}+ \\
& p\left(\mathbf{o}_{1: 2}, h_{1}=2, h_{2}=1 \mid \Theta^{\prime}\right) \log \pi_{2}+ \\
& p\left(\mathbf{o}_{1: 2}, h_{1}=2, h_{2}=2 \mid \Theta^{\prime}\right) \log \pi_{2}= \\
& p\left(\mathbf{o}_{1: 2}, h_{1}=1 \mid \Theta^{\prime}\right) \log \pi_{1}+p\left(\mathbf{o}_{1: 2}, h_{1}=2 \mid \Theta^{\prime}\right) \log \pi_{2}= \\
& \sum_{i=1}^{2} p\left(\mathbf{o}_{1: 2}, h_{1}=i \mid \Theta^{\prime}\right) \log \pi_{i}
\end{aligned}
$$


Therefore, we can avoid summing over all possible sequences of hidden states and rewrite the first term of Equation D.1 in the form of Equation D.3.

$$
\sum_{h_{1: T} \in \mathcal{H}} p\left(\mathbf{o}_{1: T}, h_{1: T} \mid \Theta^{\prime}\right) \log \pi_{h_{1}}=\sum_{i=1}^{N} p\left(\mathbf{o}_{1: T}, h_{1}=i \mid \Theta^{\prime}\right) \log \pi_{i}
$$

We wish to find the $\pi_{i}$ that maximises this expression, under the constraint that $\sum_{i=1}^{N} \pi_{i}=1$. In Equation D.4 we therefore create a new function $f$ with the addition of the Lagrange multiplier $\lambda$, using the aforementioned constraint.

$$
f=\sum_{i=1}^{N} p\left(\mathbf{o}_{1: T}, h_{1}=i \mid \Theta^{\prime}\right) \log \pi_{i}-\lambda\left(\sum_{i=1}^{N} \pi_{i}-1\right)
$$

The derivative of $f$ with respect to $\pi_{i}$ is given in Equation D.5.

$$
\begin{aligned}
\frac{\partial f}{\partial \pi_{i}}= & \frac{\partial}{\partial \pi_{i}}\left(\sum_{i=1}^{N} p\left(\mathbf{o}_{1: T}, h_{1}=i \mid \Theta^{\prime}\right) \log \pi_{i}-\lambda\left(\sum_{i=1}^{N} \pi_{i}-1\right)\right)= \\
& \frac{\partial}{\partial \pi_{i}}\left(\sum_{i=1}^{N} p\left(\mathbf{o}_{1: T}, h_{1}=i \mid \Theta^{\prime}\right) \log \pi_{i}-\sum_{i=1}^{N} \lambda \pi_{i}+\lambda\right)= \\
& \frac{\partial}{\partial \pi_{i}}\left(\sum_{i=1}^{N}\left[p\left(\mathbf{o}_{1: T}, h_{1}=i \mid \Theta^{\prime}\right) \log \pi_{i}-\lambda \pi_{i}\right]\right)+\frac{\partial}{\partial \pi_{i}} \lambda
\end{aligned}
$$

From Equation D.5 we can identify an expression for each partial derivative by realising that for, e.g. $\pi_{1}$, only the part of the sum concerning $i=1$ will be nonzero in the partial derivative, thus we can remove all of the summands where $i \neq 1$. The partial derivatives are given in Equation D.6.

$$
\begin{aligned}
& \frac{\partial}{\partial \pi_{1}}\left(p\left(\mathbf{o}_{1: T}, h_{1}=1 \mid \Theta^{\prime}\right) \log \pi_{1}-\lambda \pi_{1}\right)= \\
& \frac{1}{\pi_{1}} p\left(\mathbf{o}_{1: T}, h_{1}=1 \mid \Theta^{\prime}\right)-\lambda \\
& \vdots \\
& \frac{\partial}{\partial \pi_{N}}\left(p\left(\mathbf{o}_{1: T}, h_{1}=N \mid \Theta^{\prime}\right) \log \pi_{N}-\lambda \pi_{N}\right)= \\
& \frac{1}{\pi_{N}} p\left(\mathbf{o}_{1: T}, h_{1}=N \mid \Theta^{\prime}\right)-\lambda
\end{aligned}
$$


Any stationary point of the Lagrangian function is a stationary point of the original function, subject to the constraints. Therefore each partial derivative must be zero, thus from the last row of Equation D.6 we can conclude that for $1 \leqslant i \leqslant N$ we must have $\frac{1}{\pi_{i}} p\left(\mathbf{o}_{1: T}, h_{1}=i \mid \Theta^{\prime}\right)-\lambda=0$. This implies that $p\left(\mathbf{o}_{1: T}, h_{1}=i \mid \Theta^{\prime}\right) / \lambda=\pi_{i}$, and since $\sum_{i=1}^{N} \pi_{i}=1$, we have that $\lambda=\sum_{i=1}^{N} p\left(\mathbf{o}_{1: T}, h_{1}=i \mid \Theta^{\prime}\right)=p\left(\mathbf{o}_{1: T} \mid \Theta^{\prime}\right)$. Therefore, the choice of $\pi_{i}$ that maximises the $Q$ function is given by Equation D.7.

$$
\pi_{i}=\frac{p\left(\mathbf{o}_{1: T}, h_{1}=i \mid \Theta^{\prime}\right)}{p\left(\mathbf{o}_{1: T} \mid \Theta^{\prime}\right)}
$$

\section{D.1.2 State transition distribution}

The second term of Equation D.1 can, in a similar way as the first term, be considered a marginalisation but for each time $t$ rather than just $t=1$. To show this we can expand our example from before, having $\mathcal{H}=\{\{1,1,1\},\{1,1,2\}, \ldots,\{2,2,2\}\}$, gives us Equation D.8. 


$$
\begin{aligned}
& \sum_{h_{1: 3} \in \mathcal{H}} p\left(\mathbf{o}_{1: 3}, h_{1: 3} \mid \Theta^{\prime}\right) \sum_{t=2}^{T} \log a_{h_{t-1}} h_{t} z_{t-1}= \\
& p\left(\mathbf{o}_{1: 3}, h_{1}=1, h_{2}=1, h_{3}=1 \mid \Theta^{\prime}\right)\left(\log a_{11 z_{1}}+\log a_{11 z_{2}}\right)+ \\
& p\left(\mathbf{o}_{1: 3}, h_{1}=1, h_{2}=1, h_{3}=2 \mid \Theta^{\prime}\right)\left(\log a_{11 z_{1}}+\log a_{12 z_{2}}\right)+ \\
& p\left(\mathbf{o}_{1: 3}, h_{1}=1, h_{2}=2, h_{3}=1 \mid \Theta^{\prime}\right)\left(\log a_{12 z_{1}}+\log a_{21 z_{2}}\right)+ \\
& p\left(\mathbf{o}_{1: 3}, h_{1}=1, h_{2}=2, h_{3}=2 \mid \Theta^{\prime}\right)\left(\log a_{12 z_{1}}+\log a_{22 z_{2}}\right)+ \\
& p\left(\mathbf{o}_{1: 3}, h_{1}=2, h_{2}=1, h_{3}=1 \mid \Theta^{\prime}\right)\left(\log a_{21 z_{1}}+\log a_{11 z_{2}}\right)+ \\
& p\left(\mathbf{o}_{1: 3}, h_{1}=2, h_{2}=1, h_{3}=2 \mid \Theta^{\prime}\right)\left(\log a_{21 z_{1}}+\log a_{12 z_{2}}\right)+ \\
& p\left(\mathbf{o}_{1: 3}, h_{1}=2, h_{2}=2, h_{3}=1 \mid \Theta^{\prime}\right)\left(\log a_{22 z_{1}}+\log a_{21 z_{2}}\right)+ \\
& p\left(\mathbf{o}_{1: 3}, h_{1}=2, h_{2}=2, h_{3}=2 \mid \Theta^{\prime}\right)\left(\log a_{22 z_{1}}+\log a_{22 z_{2}}\right)= \\
& \log a_{11 z_{1}}\left(p\left(\mathbf{o}_{1: 3}, h_{1}=1, h_{2}=1, h_{3}=1 \mid \Theta^{\prime}\right)+\right. \\
& \left.p\left(\mathbf{o}_{1: 3}, h_{1}=1, h_{2}=1, h_{3}=2 \mid \Theta^{\prime}\right)\right)+ \\
& \log a_{11 z_{2}}\left(p\left(\mathbf{o}_{1: 3}, h_{1}=1, h_{2}=1, h_{3}=1 \mid \Theta^{\prime}\right)+\right. \\
& \left.p\left(\mathbf{o}_{1: 3}, h_{1}=2, h_{2}=1, h_{3}=1 \mid \Theta^{\prime}\right)\right)+ \\
& \vdots \\
& \log a_{22 z_{2}}\left(p\left(\mathbf{o}_{1: 3}, h_{1}=1, h_{2}=2, h_{3}=2 \mid \Theta^{\prime}\right)+\right. \\
& \left.p\left(\mathbf{o}_{1: 3}, h_{1}=2, h_{2}=2, h_{3}=2 \mid \Theta^{\prime}\right)\right)= \\
& \log a_{11 z_{1}} p\left(\mathbf{o}_{1: 3}, h_{1}=1, h_{2}=1 \mid \Theta^{\prime}\right)+ \\
& \log a_{11 z_{2}} p\left(\mathbf{o}_{1: 3}, h_{2}=1, h_{3}=1 \mid \Theta^{\prime}\right)+\cdots \\
& \ldots+\log a_{22 z_{2}} p\left(\mathbf{o}_{1: 3}, h_{2}=2, h_{3}=2 \mid \Theta^{\prime}\right)= \\
& \sum_{i=1}^{2} \sum_{j=1}^{2} \sum_{t=2}^{3} p\left(\mathbf{o}_{1: 3}, h_{t-1}=i, h_{t}=j \mid \Theta^{\prime}\right) \log a_{i j z_{t-1}}
\end{aligned}
$$

Following this example we can again avoid summing over all possible sequences of hidden states, and rewrite the second term of Equation D.1 in the form of Equation D.9.

$$
\begin{aligned}
& \sum_{h_{1: T} \in \mathcal{H}} p\left(\mathbf{o}_{1: T}, h_{1: T} \mid \Theta^{\prime}\right) \sum_{t=2}^{T} \log a_{h_{t-1} h_{t} z_{t-1}}= \\
& \sum_{i=1}^{N} \sum_{j=1}^{N} \sum_{t=2}^{T} p\left(\mathbf{o}_{1: T}, h_{t-1}=i, h_{t}=j \mid \Theta^{\prime}\right) \log a_{i j z_{t-1}}
\end{aligned}
$$


For each $i$ in $(1 \leqslant i \leqslant N)$ and $k$ in $\left(1 \leqslant k \leqslant S^{Z}\right)$ where $S^{Z}$ represents the number of states of $Z$, it must be the case that $\sum_{j=1}^{N} a_{i j k}=1$. We incorporate this constraint using Lagrange multipliers, and create the function $f$ in Equation D.10

$$
\begin{aligned}
& f=\sum_{i=1}^{N} \sum_{j=1}^{N} \sum_{t=2}^{T} p\left(\mathbf{o}_{1: T}, h_{t-1}=i, h_{t}=j \mid \Theta^{\prime}\right) \log a_{i j z_{t-1}}- \\
& \sum_{i=1}^{N} \sum_{k=1}^{S^{Z}} \lambda_{i k}\left(\sum_{j=1}^{N} a_{i j k}-1\right)
\end{aligned}
$$

The derivative of $f$ with respect to $a_{i j k}$ is given in Equation D.11

$$
\begin{aligned}
& \frac{\partial f}{\partial a_{i j k}}=\frac{\partial}{\partial a_{i j k}}\left(\sum_{i=1}^{N} \sum_{j=1}^{N} \sum_{t=2}^{T} p\left(\mathbf{o}_{1: T}, h_{t-1}=i, h_{t}=j \mid \Theta^{\prime}\right) \times\right. \\
& \left.\log a_{i j z_{t-1}}-\sum_{i=1}^{N} \sum_{k=1}^{S^{Z}} \lambda_{i k}\left(\sum_{j=1}^{N} a_{i j k}-1\right)\right)= \\
& \frac{\partial}{\partial a_{i j k}}\left(\sum_{i=1}^{N} \sum_{j=1}^{N} \sum_{t=2}^{T} p\left(\mathbf{o}_{1: T}, h_{t-1}=i, h_{t}=j \mid \Theta^{\prime}\right) \times\right. \\
& \left.\log a_{i j z_{t-1}}-\sum_{i=1}^{N} \sum_{k=1}^{S^{Z}} \sum_{j=1}^{N} \lambda_{i k} a_{i j k}+\sum_{i=1}^{N} \sum_{k=1}^{S^{Z}} \lambda_{i k}\right)= \\
& \frac{\partial}{\partial a_{i j k}}\left(\sum_{i=1}^{N} \sum_{j=1}^{N} \sum_{t=2}^{T} p\left(\mathbf{o}_{1: T}, h_{t-1}=i, h_{t}=j \mid \Theta^{\prime}\right) \times\right. \\
& \left.\log a_{i j z_{t-1}}-\sum_{i=1}^{N} \sum_{k=1}^{S^{Z}} \sum_{j=1}^{N} \lambda_{i k} a_{i j k}\right)+\frac{\partial}{\partial a_{i j k}}\left(\sum_{i=1}^{N} \sum_{k=1}^{S^{Z}} \lambda_{i k}\right.
\end{aligned}
$$


We can then write each partial derivative on its own by considering each combination of $i, j$ and $k$ separately, as shown in Equation D.12.

$$
\begin{gathered}
\frac{\partial}{\partial a_{111}}\left(\sum_{t=2}^{T} p\left(\mathbf{o}_{1: T}, h_{t-1}=1, h_{t}=1 \mid \Theta^{\prime}\right) \times\right. \\
\left.\log a_{11 z_{t-1}}-\lambda_{11} a_{111}\right) \\
\vdots \\
\frac{\partial}{\partial a_{N N S^{Z}}}\left(\sum_{t=2}^{T} p\left(\mathbf{o}_{1: T}, h_{t-1}=N, h_{t}=N \mid \Theta^{\prime}\right) \times\right. \\
\left.\log a_{N N z_{t-1}}-\lambda_{N N} a_{N N S^{Z}}\right)
\end{gathered}
$$

Expanding the sum in the partial derivative for $a_{111}$ gives us the summands in Equation D.13.

$$
\begin{aligned}
& \frac{\partial}{\partial a_{111}}\left(\sum_{t=2}^{T} p\left(\mathbf{o}_{1: T}, h_{t-1}=1, h_{t}=1 \mid \Theta^{\prime}\right) \log a_{11 z_{t-1}}-\lambda_{11} a_{111}\right)= \\
& \frac{\partial}{\partial a_{111}} p\left(\mathbf{o}_{1: T}, h_{1}=1, h_{2}=1 \mid \Theta^{\prime}\right) \log a_{11 z_{1}}+ \\
& \frac{\partial}{\partial a_{111}} p\left(\mathbf{o}_{1: T}, h_{1}=1, h_{2}=1 \mid \Theta^{\prime}\right) \log a_{11 z_{2}}+ \\
& \vdots \\
& \frac{\partial}{\partial a_{111}} p\left(\mathbf{o}_{1: T}, h_{T-1}=1, h_{T}=1 \mid \Theta^{\prime}\right) \log a_{11 z_{T-1}}- \\
& \frac{\partial}{\partial a_{111}} \lambda_{11} a_{111}
\end{aligned}
$$


From Equation D.13 we can see that for $t$ where $z_{t-1} \neq 1$, the term will be 0 after derivation with respect to $a_{111}$. Therefore, if we let $\delta\left(z_{t-1}=1\right)$ be one when $z_{t-1}=1$ and zero otherwise, we get Equation D.14.

$$
\begin{aligned}
& \frac{\partial}{\partial a_{111}}\left(\sum_{t=2}^{T} p\left(\mathbf{o}_{1: T}, h_{t-1}=1, h_{t}=1 \mid \Theta^{\prime}\right) \log a_{11 z_{t-1}}-\lambda_{11} a_{111}\right)= \\
& \frac{\partial}{\partial a_{111}}\left(\sum_{t=2}^{T} p\left(\mathbf{o}_{1: T}, h_{t-1}=1, h_{t}=1 \mid \Theta^{\prime}\right) \log a_{11 z_{t-1}}\right)- \\
& \frac{\partial}{\partial a_{111}} \lambda_{11} a_{111}= \\
& \frac{1}{a_{111}} \sum_{t=2}^{T} p\left(\mathbf{o}_{1: T}, h_{t-1}=1, h_{t}=1 \mid \Theta^{\prime}\right) \delta\left(z_{t-1}=1\right)-\lambda_{11}
\end{aligned}
$$

We therefore have that $a_{111}=\frac{\sum_{t=2}^{T} p\left(\mathbf{o}_{1: T}, h_{t-1}=1, h_{t}=1 \mid \Theta^{\prime}\right) \delta\left(z_{t-1}=1\right)}{\lambda_{11}}$, and since $\sum_{j=1}^{N} a_{1 j 1}=1$ we get $\lambda_{11}=\sum_{j=1}^{N} \sum_{t=2}^{T} p\left(\mathbf{o}_{1: T}, h_{t-1}=1, h_{t}=\right.$ $\left.j \mid \Theta^{\prime}\right) \delta\left(z_{t-1}=1\right)=\sum_{t=2}^{T} p\left(\mathbf{o}_{1: T}, h_{t-1}=1 \mid \Theta^{\prime}\right) \delta\left(z_{t-1}=1\right)$. By generalising what we have done for $a_{111}$ to any $a_{i j k}$, we find that the $a_{i j k}$ that maximises the $Q$ function is given by Equation D.15.

$$
a_{i j k}=\frac{\sum_{t=2}^{T} p\left(\mathbf{o}_{1: T}, h_{t-1}=i, h_{t}=j \mid \Theta^{\prime}\right) \delta\left(z_{t-1}=k\right)}{\sum_{t=2}^{T} p\left(\mathbf{o}_{1: T}, h_{t-1}=i \mid \Theta^{\prime}\right) \delta\left(z_{t-1}=k\right)}
$$

\section{D.1.3 Observational model distribution}

From the third term in Equation D.1 we can see that it is possible to treat each observed variable separately. Therefore, we shall consider the case where $i=1$, from which the rest of the observational variables follow. As was the case with the initial state distribution and transition state distribution, we start by avoiding the summation over every possible hidden 
state sequence via marginalisation. We revisit the example with $\mathcal{H}=$ $\{\{1,1\},\{1,2\},\{2,1\},\{2,2\}\}$, and expand the summations in Equation D.16.

$$
\begin{aligned}
& \sum_{h_{1: 2} \in \mathcal{H}} p\left(\mathbf{o}_{1: 2}, h_{1: 2} \mid \Theta^{\prime}\right) \sum_{t=1}^{2} \log b_{h_{t}}^{1}\left(\mathbf{o}_{t}\right)= \\
& p\left(\mathbf{o}_{1: 2}, h_{1}=1, h_{2}=1 \mid \Theta^{\prime}\right)\left(\log b_{1}^{1}\left(\mathbf{o}_{1}\right)+\log b_{1}^{1}\left(\mathbf{o}_{2}\right)\right)+ \\
& p\left(\mathbf{o}_{1: 2}, h_{1}=1, h_{2}=2 \mid \Theta^{\prime}\right)\left(\log b_{1}^{1}\left(\mathbf{o}_{1}\right)+\log b_{2}^{1}\left(\mathbf{o}_{2}\right)\right)+ \\
& p\left(\mathbf{o}_{1: 2}, h_{1}=2, h_{2}=1 \mid \Theta^{\prime}\right)\left(\log b_{2}^{1}\left(\mathbf{o}_{1}\right)+\log b_{1}^{1}\left(\mathbf{o}_{2}\right)\right)+ \\
& p\left(\mathbf{o}_{1: 2}, h_{1}=2, h_{2}=2 \mid \Theta^{\prime}\right)\left(\log b_{2}^{1}\left(\mathbf{o}_{1}\right)+\log b_{2}^{1}\left(\mathbf{o}_{2}\right)\right)= \\
& \log b_{1}^{1}\left(\mathbf{o}_{1}\right) \times \\
& \left(p\left(\mathbf{o}_{1: 2}, h_{1}=1, h_{2}=1 \mid \Theta^{\prime}\right)+p\left(\mathbf{o}_{1: 2}, h_{1}=1, h_{2}=2 \mid \Theta^{\prime}\right)\right)+ \\
& \log b_{1}^{1}\left(\mathbf{o}_{2}\right) \times \\
& \left(p\left(\mathbf{o}_{1: 2}, h_{1}=1, h_{2}=1 \mid \Theta^{\prime}\right)+p\left(\mathbf{o}_{1: 2}, h_{1}=2, h_{2}=1 \mid \Theta^{\prime}\right)\right)+ \\
& \log b_{2}^{1}\left(\mathbf{o}_{1}\right) \times \\
& \left(p\left(\mathbf{o}_{1: 2}, h_{1}=2, h_{2}=1 \mid \Theta^{\prime}\right)+p\left(\mathbf{o}_{1: 2}, h_{1}=2, h_{2}=2 \mid \Theta^{\prime}\right)\right)+ \\
& \log b_{2}^{1}\left(\mathbf{o}_{2}\right) \times \\
& \left(p\left(\mathbf{o}_{1: 2}, h_{1}=1, h_{2}=2 \mid \Theta^{\prime}\right)+p\left(\mathbf{o}_{1: 2}, h_{1}=2, h_{2}=2 \mid \Theta^{\prime}\right)\right)= \\
& \log b_{1}^{1}\left(\mathbf{o}_{1}\right) p\left(\mathbf{o}_{1: 2}, h_{1}=1 \mid \Theta^{\prime}\right)+\log b_{1}^{1}\left(\mathbf{o}_{2}\right) p\left(\mathbf{o}_{1: 2}, h_{2}=1 \mid \Theta^{\prime}\right)+ \\
& \log b_{2}^{1}\left(\mathbf{o}_{1}\right) p\left(\mathbf{o}_{1: 2}, h_{1}=2 \mid \Theta^{\prime}\right)+\log b_{2}^{1}\left(\mathbf{o}_{2}\right) p\left(\mathbf{o}_{1: 2}, h_{2}=2 \mid \Theta^{\prime}\right)= \\
& \sum_{j=1}^{2} \sum_{t=1}^{2} p\left(\mathbf{o}_{1: 2}, h_{t}=j \mid \Theta^{\prime}\right) \log b_{j}^{1}\left(\mathbf{o}_{t}\right)
\end{aligned}
$$

Thus, we again avoid summing over every possible hidden state sequence, and therefore for observed variable $i=1$ we have Equation D.17.

$$
\begin{aligned}
& \sum_{h_{1: T} \in \mathcal{H}} \sum_{t=1}^{T} p\left(\mathbf{o}_{1: T}, h_{1: T} \mid \Theta^{\prime}\right) \log b_{h_{t}}^{1}\left(\mathbf{o}_{t}\right)= \\
& \sum_{j=1}^{N} \sum_{t=1}^{T} p\left(\mathbf{o}_{1: T}, h_{t}=j \mid \Theta^{\prime}\right) \log b_{j}^{1}\left(\mathbf{o}_{t}\right)
\end{aligned}
$$

The observed variable under consideration has $S^{1}$ states, which we will index by $l$. For each hidden state $j \in[1, N]$ and parent configuration $k \in\left[1, K^{j}\right]$ of the observed variable (where $K^{j}$ is the number of parent configurations for the observed variable under hidden state $j$ ), we know that summing 
over all $S^{1}$ states will result in unity. If we let $b_{j k l}^{1}$ represent the probability of state $l$ given hidden state $j$ and parent configuration $k$, then we have the constraints that $\sum_{l=1}^{S^{1}} b_{j k l}^{1}=1$.

As before, in Equation D.18 we create a new function $f$ by introducing Lagrange multipliers using the aforementioned constraints.

$$
\begin{aligned}
& f=\sum_{j=1}^{N} \sum_{t=1}^{T} p\left(\mathbf{o}_{1: T}, h_{t}=j \mid \Theta^{\prime}\right) \log b_{j}^{1}\left(\mathbf{o}_{t}\right)- \\
& \sum_{j=1}^{N} \sum_{k=1}^{K^{j}} \lambda_{j k}\left(\sum_{l=1}^{S^{1}} b_{j k l}^{1}-1\right)
\end{aligned}
$$

The derivative of $f$ is then given by Equation D.19.

$$
\begin{aligned}
& \frac{\partial f}{\partial b_{j k l}^{1}}= \\
& \frac{\partial}{\partial b_{j k l}^{1}}\left(\sum_{j=1}^{N} \sum_{t=1}^{T} p\left(\mathbf{o}_{1: T}, h_{t}=j \mid \Theta^{\prime}\right) \log b_{j}^{1}\left(\mathbf{o}_{t}\right)-\right. \\
& \sum_{j=1}^{N} \sum_{k=1}^{K^{j}} \lambda_{j k}\left(\sum_{l=1}^{S^{1}} b_{j k l}^{1}-1\right)= \\
& \frac{\partial}{\partial b_{j k l}^{1}}\left(\sum_{j=1}^{N} \sum_{t=1}^{T} p\left(\mathbf{o}_{1: T}, h_{t}=j \mid \Theta^{\prime}\right) \log b_{j}^{1}\left(\mathbf{o}_{t}\right)-\right. \\
& \sum_{j=1}^{N} \sum_{k=1}^{K^{j}} \sum_{l=1}^{S^{1}} \lambda_{j k} b_{j k l}^{1}+\sum_{j=1}^{N} \sum_{k=1}^{K^{j}} \lambda_{j k}= \\
& \frac{\partial}{\partial b_{j k l}^{1}}\left(\sum_{j=1}^{N} \sum_{t=1}^{T} p\left(\mathbf{o}_{1: T}, h_{t}=j \mid \Theta^{\prime}\right) \log b_{j}^{1}\left(\mathbf{o}_{t}\right)-\right. \\
& \sum_{j=1}^{N} \sum_{k=1}^{K^{j}} \sum_{l=1}^{S^{1}} \lambda_{j k} b_{j k l}^{1}+\frac{\partial}{\partial b_{j k k}^{1}}\left(\sum_{j=1}^{N} \sum_{k=1}^{K^{j}} \lambda_{j k}\right.
\end{aligned}
$$


From Equation D.19 we can extract each partial derivative on its own in Equation D.20.

$$
\begin{aligned}
& \frac{\partial}{\partial b_{111}^{1}}\left(\sum_{t=1}^{T} p\left(\mathbf{o}_{1: T}, h_{t}=1 \mid \Theta^{\prime}\right) \log b_{1}^{1}\left(\mathbf{o}_{t}\right)-\lambda_{11} b_{111}^{1}\right) \\
& \vdots \\
& \frac{\partial}{\partial b_{N K^{N} S^{1}}^{1}}\left(\sum_{t=1}^{T} p\left(\mathbf{o}_{1: T}, h_{t}=N \mid \Theta^{\prime}\right) \log b_{N}^{1}\left(\mathbf{o}_{t}\right)-\lambda_{N K^{N}} b_{N K^{N} S^{1}}^{1}\right)
\end{aligned}
$$

We shall continue in Equation D.21 by expanding the summation within the partial derivative with respect to $\partial b_{111}^{1}$, from which the rest will follow.

$$
\begin{aligned}
& \frac{\partial}{\partial b_{111}^{1}}\left(\sum_{t=1}^{T} p\left(\mathbf{o}_{1: T}, h_{t}=1 \mid \Theta^{\prime}\right) \log b_{1}^{1}\left(\mathbf{o}_{t}\right)-\lambda_{11} b_{111}^{1}\right)= \\
& \frac{\partial}{\partial b_{111}^{1}} p\left(\mathbf{o}_{1: T}, h_{1}=1 \mid \Theta^{\prime}\right) \log b_{1}^{1}\left(\mathbf{o}_{1}\right)+ \\
& \frac{\partial}{\partial b_{111}^{1}} p\left(\mathbf{o}_{1: T}, h_{2}=1 \mid \Theta^{\prime}\right) \log b_{1}^{1}\left(\mathbf{o}_{2}\right)+ \\
& \vdots \\
& \frac{\partial}{\partial b_{111}^{1}} p\left(\mathbf{o}_{1: T}, h_{T}=1 \mid \Theta^{\prime}\right) \log b_{1}^{1}\left(\mathbf{o}_{T}\right)- \\
& \frac{\partial}{\partial b_{111}^{1}} \lambda_{11} b_{111}^{1}
\end{aligned}
$$

By observing that each term $b_{1}^{1}\left(\mathbf{o}_{t}\right)$ in Equation D.21 identifies the parameter $b_{j k l}^{1}$ that is consistent with $\mathbf{o}_{t}$, then it follows that only terms $p\left(\mathbf{o}_{1: T}, h_{t}=\right.$ $\left.1 \mid \Theta^{\prime}\right) \log b_{1}^{1}\left(\mathbf{o}_{t}\right)$ in which the parameter identified is $b_{111}^{1}$ contribute to the sum once the partial derivative is taken. If we let $\delta\left(\mathbf{o}_{t}, b_{111}^{1}\right)$ be one when $b_{111}^{1}$ is identified, and zero otherwise, we get Equation D.22

$$
\begin{aligned}
& \frac{\partial}{\partial b_{111}^{1}}\left(\sum_{t=1}^{T} p\left(\mathbf{o}_{1: T}, h_{t}=1 \mid \Theta^{\prime}\right) \log b_{1}^{1}\left(\mathbf{o}_{t}\right)-\lambda_{11} b_{111}^{1}\right)= \\
& \frac{1}{b_{111}^{1}} \sum_{t=1}^{T} p\left(\mathbf{o}_{1: T}, h_{t}=1 \mid \Theta^{\prime}\right) \delta\left(\mathbf{o}_{t}, b_{111}^{1}\right)-\lambda_{11}
\end{aligned}
$$


The condition for stationary points is given by setting each partial derivative to zero, thus continuing the example we have $b_{111}^{1}=$ $\frac{\sum_{t=1}^{T} p\left(\mathbf{o}_{1: T}, h_{t}=1 \mid \Theta^{\prime}\right) \delta\left(\mathbf{o}_{t}, b_{111}^{1}\right)}{\lambda_{11}}$. Summing over each state $S^{1}$ gives $\sum_{l=1}^{S} b_{11 l}^{1}=$ $\frac{\sum_{l=1}^{S} \sum_{t=1}^{T} p\left(\mathbf{o}_{1: T}, h_{t}=1 \mid \Theta^{\prime}\right) \delta\left(\mathbf{o}_{t}, b_{11 l}^{1}\right)}{\lambda_{11}}=1$. From here we can form an expression for $\lambda_{11}$ and solve it according to Equation D.23

$$
\begin{aligned}
& \lambda_{11}=\sum_{l=1}^{S^{1}} \sum_{t=1}^{T} p\left(\mathbf{o}_{1: T}, h_{t}=1 \mid \Theta^{\prime}\right) \delta\left(\mathbf{o}_{t}, b_{11 l}^{1}\right)= \\
& \sum_{t=1}^{T} p\left(\mathbf{o}_{1: T}, h_{t}=1 \mid \Theta^{\prime}\right) \delta\left(\mathbf{o}_{t}, b_{111}^{1}\right)+\ldots+ \\
& \sum_{t=1}^{T} p\left(\mathbf{o}_{1: T}, h_{t}=1 \mid \Theta^{\prime}\right) \delta\left(\mathbf{o}_{t}, b_{11 S^{1}}^{1}\right)= \\
& \sum_{t=1}^{T} p\left(\mathbf{o}_{1: T}, h_{t}=1 \mid \Theta^{\prime}\right) \delta\left(\mathbf{o}_{t}, b_{11}^{1}\right)
\end{aligned}
$$

In the last row of Equation D.23 we let $\delta\left(\mathbf{o}_{t}, b_{j k}^{i}\right)$ be one only when $\mathbf{o}_{t}$ is consistent with parent configuration $k$ for variable $i$ under hidden state $j$, else it will be zero. It follows from the fact that we are summing over all $S^{1}$ states that the observed variable takes, and since for each configuration of its parents it must take on a state, that we are essentially counting the number of times we are observing the parent state.

We therefore find that the value for parameter $b_{111}^{1}$ that maximises the $Q$ function is given by Equation D.24 and in general for each observable variable and its respective parameters we have Equation D.25.

$$
\begin{gathered}
b_{111}^{1}=\frac{\sum_{t=1}^{T} p\left(\mathbf{o}_{1: T}, h_{t}=1 \mid \Theta^{\prime}\right) \delta\left(\mathbf{o}_{t}, b_{111}^{1}\right)}{\sum_{t=1}^{T} p\left(\mathbf{o}_{1: T}, h_{t}=1 \mid \Theta^{\prime}\right) \delta\left(\mathbf{o}_{t}, b_{11}^{1}\right)} \\
b_{j k l}^{i}=\frac{\sum_{t=1}^{T} p\left(\mathbf{o}_{1: T}, h_{t}=j \mid \Theta^{\prime}\right) \delta\left(\mathbf{o}_{t}, b_{j k l}^{i}\right)}{\sum_{t=1}^{T} p\left(\mathbf{o}_{1: T}, h_{t}=j \mid \Theta^{\prime}\right) \delta\left(\mathbf{o}_{t}, b_{j k}^{i}\right)}
\end{gathered}
$$




\section{D.2 Inference}

The first desired quantity that we shall consider will be $p\left(O_{1: T}, H_{t} \mid \Theta^{\prime}\right)$, which we shall refer to as $\gamma\left(H_{T}\right)$ and expand according to Equation D.26.

$$
\begin{aligned}
\gamma\left(H_{t}\right)= & p\left(O_{1: T}, H_{t} \mid \Theta^{\prime}\right)=p\left(O_{1: t}, O_{t+1: T}, H_{t} \mid \Theta^{\prime}\right)= \\
& p\left(O_{t+1: T} \mid O_{1: t}{ }^{O_{t}}{ }^{\prime} H_{t}, \Theta^{\prime}\right) p\left(O_{1: t}, H_{t} \mid \Theta^{\prime}\right)= \\
& p\left(O_{t+1: T} \mid O_{t}, H_{t}, \Theta^{\prime}\right) p\left(O_{1: t}, H_{t} \mid \Theta^{\prime}\right)
\end{aligned}
$$

In the second row of Equation D.26 we notice that we cannot cancel out all the observations in the first factor, as we have not conditioned on $H_{t+1}$ and due to the $Z$ variable we cannot assume independence.

The final two factors of Equation D.26 can efficiently be computed. The joint probability of the state of $H_{t}$ and the observations $O_{1: t}$ is given by Equation D.27, and the conditional probability of the observations $O_{t+1: T}$ given the state of $H_{t}$ and the observation $O_{t}$ is given by Equation D.28.

$$
\begin{aligned}
& \alpha\left(H_{t}\right)=p\left(H_{t}, O_{1: t} \mid \Theta^{\prime}\right)=\sum_{H_{t-1}} p\left(H_{t}, H_{t-1}, O_{1: t-1}, O_{t} \mid \Theta^{\prime}\right)= \\
& \sum_{H_{t-1}} p\left(O_{t} \mid H_{t}, H_{t-1}, O_{1: t-1}, \Theta^{\prime}\right) p\left(H_{t}, H_{t-1}, O_{1: t-1} \mid \Theta^{\prime}\right)= \\
& \sum_{H_{t-1}} p\left(O_{t} \mid H_{t}, \Theta^{\prime}\right) p\left(H_{t} \mid H_{t-1}, O_{1: t-1} O_{t-1}, \Theta^{\prime}\right) \times \\
& p\left(H_{t-1}, O_{1: t-1} \mid \Theta^{\prime}\right)= \\
& p\left(O_{t} \mid H_{t}, \Theta^{\prime}\right) \sum_{H_{t-1}} p\left(H_{t} \mid H_{t-1}, O_{t-1}, \Theta^{\prime}\right) \alpha\left(H_{t-1}\right)= \\
& \prod_{i=1}^{M} b_{H_{t}}^{i}\left(O_{t}\right) \sum_{H_{t-1}} a_{H_{t-1} H_{t} Z_{t-1}} \alpha\left(H_{t-1}\right)
\end{aligned}
$$




$$
\begin{aligned}
& \beta\left(H_{t}\right)=p\left(O_{t+1: T} \mid H_{t}, O_{t}, \Theta^{\prime}\right)= \\
& \sum_{H_{t+1}} p\left(O_{t+1}, O_{t+2: T}, H_{t+1} \mid H_{t}, O_{t}, \Theta^{\prime}\right)= \\
& \sum_{H_{t+1}} p\left(O_{t+2: T} \mid H_{t+1}, O_{t+1}, H_{t}, \varnothing_{t}, \Theta^{\prime}\right) \times \\
& p\left(O_{t+1}, H_{t+1} \mid H_{t}, O_{t}, \Theta^{\prime}\right)= \\
& \sum_{H_{t+1}} p\left(O_{t+2: T} \mid H_{t+1}, O_{t+1}, \Theta^{\prime}\right) p\left(O_{t+1} \mid H_{t+1}, H_{t}, \varnothing_{t}, \Theta^{\prime}\right) \times \\
& p\left(H_{t+1} \mid H_{t}, O_{t}, \Theta^{\prime}\right)= \\
& \sum_{H_{t+1}} \beta\left(H_{t+1}\right) p\left(O_{t+1} \mid H_{t+1}, \Theta^{\prime}\right) p\left(H_{t+1} \mid H_{t}, O_{t}, \Theta^{\prime}\right)= \\
& \sum_{H_{t+1}} \beta\left(H_{t+1}\right) \prod_{i=1}^{M} b_{H_{t+1}}^{i}\left(O_{t+1}\right) a_{H_{t} H_{t+1} Z_{t}}
\end{aligned}
$$

Using this new notation, we can write $\gamma\left(H_{t}\right)=\alpha\left(H_{t}\right) \beta\left(H_{t}\right)$. Noticing that $\sum_{i=1}^{N} \gamma\left(H_{t}=i\right)=p\left(O_{1: T} \mid \Theta^{\prime}\right)$, we can readily compute Equation D.7 and Equation D.25 since both $\alpha$ and $\beta$ are expressed in known quantities (under the parameters $\Theta^{\prime}$ ).

The second quantity that we need to be able to compute, in order to compute Equation D.15, is $p\left(O_{1: T}, H_{t-1}, H_{t} \mid \Theta^{\prime}\right)$, which we shall refer to as $\xi\left(H_{t-1}, H_{t}\right)$. We expand this quantity in Equation D.29, and express it in terms of known quantities.

$$
\begin{aligned}
& \xi\left(H_{t-1}, H_{t}\right)=p\left(O_{1: T}, H_{t-1}, H_{t} \mid \Theta^{\prime}\right)= \\
& p\left(O_{1: t-1}, O_{t}, O_{t+1: T}, H_{t-1}, H_{t} \mid \Theta^{\prime}\right)= \\
& p\left(O_{t+1: T} \mid \Theta_{1: t-1}, O_{t}, H_{t-1}, H_{t}, \Theta^{\prime}\right) p\left(O_{1: t-1}, O_{t}, H_{t-1}, H_{t} \mid \Theta^{\prime}\right)= \\
& p\left(O_{t+1: T} \mid O_{t}, H_{t}, \Theta^{\prime}\right) p\left(O_{t} \mid \Theta_{1: t-1}, H_{t-1}, H_{t}, \Theta^{\prime}\right) \times \\
& p\left(O_{1: t-1}, H_{t-1}, H_{t} \mid \Theta^{\prime}\right)= \\
& p\left(O_{t+1: T} \mid O_{t}, H_{t}, \Theta^{\prime}\right) p\left(O_{t} \mid H_{t}, \Theta^{\prime}\right) \times \\
& p\left(H_{t} \mid O_{1: t-1} O_{t-1}, H_{t-1}, \Theta^{\prime}\right) p\left(O_{1: t-1}, H_{t-1} \mid \Theta^{\prime}\right)= \\
& p\left(O_{t+1: T} \mid O_{t}, H_{t}, \Theta^{\prime}\right) p\left(O_{t} \mid H_{t}, \Theta^{\prime}\right) p\left(H_{t} \mid O_{t-1}, H_{t-1}, \Theta^{\prime}\right) \times \\
& p\left(O_{1: t-1}, H_{t-1} \mid \Theta^{\prime}\right)= \\
& \beta\left(H_{t}\right) \prod_{i=1}^{M} b_{H_{t}}^{i}\left(O_{t}\right) a_{H_{t-1} H_{t} Z_{t-1}} \alpha\left(H_{t-1}\right)
\end{aligned}
$$


D.2. Inference 

Department of Computer and Information Science

Linköpings universitet

\section{Dissertations}

\section{Linköping Studies in Science and Technology \\ Linköping Studies in Arts and Science \\ Linköping Studies in Statistics \\ Linköping Studies in Information Science}

Linköping Studies in Science and Technology

No 14 Anders Haraldsson: A Program Manipulation System Based on Partial Evaluation, 1977, ISBN 917372-144-1.

No 17 Bengt Magnhagen: Probability Based Verification of Time Margins in Digital Designs, 1977, ISBN 91-7372157-3.

No 18 Mats Cedwall: Semantisk analys av processbeskrivningar i naturligt språk, 1977, ISBN 91-7372168-9.

No 22 Jaak Urmi: A Machine Independent LISP Compiler and its Implications for Ideal Hardware, 1978, ISBN 91-7372-188-3.

No 33 Tore Risch: Compilation of Multiple File Queries in a Meta-Database System 1978, ISBN 91- 7372-232-4.

No 51 Erland Jungert: Synthesizing Database Structures from a User Oriented Data Model, 1980, ISBN 917372-387-8.

No 54 Sture Hägglund: Contributions to the Development of Methods and Tools for Interactive Design of Applications Softw are, 1980, ISBN 91-7372-404-1.

No 55 Pär Emanuelson: Performance Enhancement in a Well-Structured Pattern Matcher through Partial Evaluation, 1980, ISBN 91-7372-403-3.

No 58 Bengt Johnsson, Bertil Andersson: The HumanComputer Interface in Commercial Systems, 1981, ISBN 91-7372-414-9.

No 69 H. Jan Komorowski: A Specification of an Abstract Prolog Machine and its Application to Partial Evaluation, 1981, ISBN 91-7372-479-3.

No 71 René Reboh: Knowledge Engineering Techniques and Tools for Expert Systems, 1981, ISBN 91-7372489-0.

No 77 Östen Oskarsson: Mechanisms of Modifiability in large Softw are Systems, 1982, ISBN 91- 7372-527-7.

No 94 Hans Lunell: Code Generator Writing Systems, 1983 ISBN 91-7372-652-4.

No 97 Andrzej Lingas: Advances in Minimum Weight Triangulation, 1983, ISBN 91-7372-660-5.

No 109 Peter Fritzson: Tow ards a Distributed Programming Environment based on Incremental Compilation, 1984, ISBN 91-7372-801-2.

No 111 Erik Tengvald: The Design of Expert Planning Systems. An Experimental Operations Planning System for Turning, 1984, ISBN 91-7372- 805-5.

No 155 Christos Levcopoulos: Heuristics for Minimum Decompositions of Polygons, 1987, ISBN 91-7870133-3.

No 165 James W. Goodwin: A Theory and System for NonMonotonic Reasoning, 1987, ISBN 91-7870-183-X.

No 170 Zebo Peng: A Formal Methodology for Automated Synthesis of VLSI Systems, 1987, ISBN 91-7870-225-9.

No 174 Johan Fagerström: A Paradigm and System for Design of Distributed Systems, 1988, ISBN 91-7870301-8.

No 192 Dimiter Driankov: Tow ards a Many Valued Logic of Quantified Belief, 1988, ISBN 91-7870-374-3.
No 213 Lin Padgham: Non-Monotonic Inheritance for an Object Oriented Knowledge Base, 1989, ISBN 917870-485-5.

No 214 Tony Larsson: A Formal Hardware Description and Verification Method, 1989, ISBN 91-7870-517-7.

No 221 Michael Reinfrank: Fundamentals and Logical Foundations of Truth Maintenance, 1989, ISBN 917870-546-0.

No 239 Jonas Löwgren: Knowledge-Based Design Support and Discourse Management in User Interface Management Systems, 1991, ISBN 91-7870-720-X.

No 244 Henrik Eriksson: Meta-Tool Support for Know ledge Acquisition, 1991, ISBN 91-7870-746-3.

No 252 Peter Eklund: An Epistemic Approach to Interactive Design in Multiple Inheritance Hierarchies, 1991, ISBN 91-7870-784-6.

No 258 Patrick Doherty: NML3 - A Non-Monotonic Formalism with Explicit Defaults, 1991, ISBN 917870-816-8

No 260 Nahid Shahmehri: Generalized Algorithmic Debugging, 1991, ISBN 91-7870-828-1.

No 264 Nils Dahlbäck: Representation of DiscourseCognitive and Computational Aspects, 1992, ISBN 91-7870-850-8.

No 265 Ulf Nilsson: Abstract Interpretations and Abstract Machines: Contributions to a Methodology for the Implementation of Logic Programs, 1992, ISBN 917870-858-3.

No 270 Ralph Rönnquist: Theory and Practice of Tensebound Object References, 1992, ISBN 91-7870-873-7.

No 273 Björn Fjellborg: Pipeline Extraction for VLSI Data Path Synthesis, 1992, ISBN 91-7870-880-X.

No 276 Staffan Bonnier: A Formal Basis for Horn Clause Logic with External Polymorphic Functions, 1992, ISBN 91-7870-896-6.

No 277 Kristian Sandahl: Developing Knowledge Management Systems with an Active Expert Methodology, 1992, ISBN 91-7870-897-4.

No 281 Christer Bäckström: Computational Complexity of Reasoning about Plans, 1992, ISBN 91-7870-979-2.

No 292 Mats Wirén: Studies in Incremental Natural Language Analysis, 1992, ISBN 91-7871-027-8.

No 297 Mariam Kamkar: Interprocedural Dynamic Slicing with Applications to Debugging and Testing, 1993, ISBN 91-7871-065-0.

No 302 Tingting Zhang: A Study in Diagnosis Using Classification and Defaults, 1993, ISBN 91-7871-078-2

No 312 Arne Jönsson: Dialogue Management for Natural Language Interfaces - An Empirical Approach, 1993, ISBN 91-7871-110-X

No 338 Simin Nadjm-Tehrani: Reactive Systems in Physical Environments: Compositional Modelling and Framework for Verification, 1994, ISBN 91-7871-237-8.

No 371 Bengt Savén: Business Models for Decision Support and Learning. A Study of Discrete-Event Manufacturing Simulation at Asea/ ABB 1968-1993, 1995, ISBN 91-7871-494-X. 
No 375 Ulf Söderman: Conceptual Modelling of Mode Switching Physical Systems, 1995, ISBN 91-7871-5164.

No 383 Andreas Kågedal: Exploiting Groundness in Logic Programs, 1995, ISBN 91-7871-538-5.

No 396 George Fodor: Ontological Control, Description, Identification and Recovery from Problematic Control Situations, 1995, ISBN 91-7871-603-9.

No 413 Mikael Pettersson: Compiling Natural Semantics, 1995, ISBN 91-7871-641-1.

No 414 Xinli Gu: RT Level Testability Improvement by Testability Analysis and Transformations, 1996, ISBN 91-7871-654-3.

No 416 Hua Shu: Distributed Default Reasoning, 1996, ISBN 91-7871-665-9.

No 429 Jaime Villegas: Simulation Supported Industrial Training from an Organisational Learning Perspective - Development and Evaluation of the SSIT Method, 1996, ISBN 91-7871-700-0.

No 431 Peter Jonsson: Studies in Action Planning: Algorithms and Complexity, 1996, ISBN 91-7871-7043 .

No 437 Johan Boye: Directional Types in Logic Programming, 1996, ISBN 91-7871-725-6.

No 439 Cecilia Sjöberg: Activities, Voices and Arenas: Participatory Design in Practice, 1996, ISBN 91-7871728-0.

No 448 Patrick Lambrix: Part-Whole Reasoning in Description Logics, 1996, ISBN 91-7871-820-1.

No 452 Kjell Orsborn: On Extensible and Object-Relational Database Technology for Finite Element Analysis Applications, 1996, ISBN 91-7871-827-9.

No 459 Olof Johansson: Development Environments for Complex Product Models, 1996, ISBN 91-7871-855-4.

No 461 Lena Strömbäck: User-Defined Constructions in Unification-Based Formalisms, 1997, ISBN 91-7871857-0.

No 462 Lars Degerstedt: Tabulation-based Logic Programming: A Multi-Level View of Query Answering, 1996, ISBN 91-7871-858-9.

No 475 Fredrik Nilsson: Strategi och ekonomisk styrning En studie av hur ekonomiska styrsystem utformas och används efter företagsförvärv, 1997, ISBN 917871-914-3.

No 480 Mikael Lindvall: An Empirical Study of Requirements-Driven Impact Analysis in Object-Oriented Softw are Evolution, 1997, ISBN 91-7871-927-5.

No 485 Göran Forslund: Opinion-Based Systems: The Cooperative Perspective on Knowledge-Based Decision Support, 1997, ISBN 91-7871-938-0.

No 494 Martin Sköld: Active Database Management Systems for Monitoring and Control, 1997, ISBN 917219-002-7.

No 495 Hans Olsén: Automatic Verification of Petri Nets in a CLP framew ork, 1997, ISBN 91-7219-011-6.

No 498 Thomas Drakengren: Algorithms and Complexity for Temporal and Spatial Formalisms, 1997, ISBN 91 7219-019-1.

No 502 Jakob Axelsson: Analysis and Synthesis of Heterogeneous Real-Time Systems, 1997, ISBN 91-7219-035-3.

No 503 Johan Ringström: Compiler Generation for DataParallel Programming Languages from Two-Level Semantics Specifications, 1997, ISBN 91-7219-045-0.

No 512 Anna Moberg: Närhet och distans - Studier av kommunikationsmönster i satellitkontor och flexibla kontor, 1997, ISBN 91-7219-119-8.
No 520 Mikael Ronström: Design and Modelling of a Parallel Data Server for Telecom Applications, 1998, ISBN 91-7219-169-4.

No 522 Niclas Ohlsson: Tow ards Effective Fault Prevention - An Empirical Study in Software Engineering, 1998, ISBN 91-7219-176-7.

No 526 Joachim Karlsson: A Systematic Approach for Prioritizing Software Requirements, 1998, ISBN 91 7219-184-8.

No 530 Henrik Nilsson: Declarative Debugging for Lazy Functional Languages, 1998, ISBN 91-7219-197-x.

No 555 Jonas Hallberg: Timing Issues in High-Level Synthesis, 1998, ISBN 91-7219-369-7.

No 561 Ling Lin: Management of 1-D Sequence Data - From Discrete to Continuous, 1999, ISBN 91-7219-402-2.

No 563 Eva L Ragnemalm: Student Modelling based on Collaborative Dialogue with a Learning Companion, 1999, ISBN 91-7219-412-X

No 567 Jörgen Lindström: Does Distance matter? On geographical dispersion in organisations, 1999, ISBN 917219-439-1.

No 582 Vanja Josifovski: Design, Implementation and Evaluation of a Distributed Mediator System for Data Integration, 1999, ISBN 91-7219-482-0.

No 589 Rita Kovordányi: Modeling and Simulating Inhibitory Mechanisms in Mental Image Reinterpretation - Towards Cooperative HumanComputer Creativity, 1999, ISBN 91-7219-506-1.

No 592 Mikael Ericsson: Supporting the Use of Design Knowledge - An Assessment of Commenting Agents, 1999, ISBN 91-7219-532-0.

No 593 Lars Karlsson: Actions, Interactions and Narratives, 1999, ISBN 91-7219-534-7.

No 594 C. G. Mikael Johansson: Social and Organizational Aspects of Requirements Engineering Methods - A practice-oriented approach, 1999, ISBN 91-7219-541$\mathrm{X}$

No 595 Jörgen Hansson: Value-Driven Multi-Class Overload Management in Real-Time Database Systems, 1999, ISBN 91-7219-542-8.

No 596 Niklas Hallberg: Incorporating User Values in the Design of Information Systems and Services in the Public Sector: A Methods Approach, 1999, ISBN 91 7219-543-6.

No 597 Vivian Vimarlund: An Economic Perspective on the Analysis of Impacts of Information Technology: From Case Studies in Health-Care towards General Models and Theories, 1999, ISBN 91-7219-544-4.

No 598 Johan Jenvald: Methods and Tools in ComputerSupported Taskforce Training, 1999, ISBN 91-7219 $547-9$

No 607 Magnus Merkel: Understanding and enhancing translation by parallel text processing, 1999, ISBN 917219-614-9.

No 611 Silvia Coradeschi: Anchoring symbols to sensory data, 1999, ISBN 91-7219-623-8.

No 613 Man Lin: Analysis and Synthesis of Reactive Systems: A Generic Layered Architecture Perspective, 1999, ISBN 91-7219-630-0.

No 618 Jimmy Tjäder: Systemimplementering i praktiken En studie av logiker i fyra projekt, 1999, ISBN 917219-657-2

No 627 Vadim Engelson: Tools for Design, Interactive Simulation, and Visualization of Object-Oriented Models in Scientific Computing, 2000, ISBN 91-7219709-9. 
No 637 Esa Falkenroth: Database Technology for Control and Simulation, 2000, ISBN 91-7219-766-8.

No 639 Per-Arne Persson: Bringing Power and Knowledge Together: Information Systems Design for Autonomy and Control in Command Work, 2000, ISBN 91-7219 796-X.

No 660 Erik Larsson: An Integrated System-Level Design for Testability Methodology, 2000, ISBN 91-7219-890-7.

No 688 Marcus Bjäreland: Model-based Execution Monitoring, 2001, ISBN 91-7373-016-5.

No 689 Joakim Gustafsson: Extending Temporal Action Logic, 2001, ISBN 91-7373-017-3.

No 720 Carl-Johan Petri: Organizational Information Provision - Managing Mandatory and Discretionary Use of Information Technology, 2001, ISBN-91-7373-1269.

No 724 Paul Scerri: Designing Agents for Systems with Adjustable Autonomy, 2001, ISBN 9173732079.

No 725 Tim Heyer: Semantic Inspection of Software Artifacts: From Theory to Practice, 2001, ISBN 91 73732087.

No 726 Pär Carlshamre: A Usability Perspective on Requirements Engineering - From Methodology to Product Development, 2001, ISBN 9173732125.

No 732 Juha Takkinen: From Information Management to Task Management in Electronic Mail, 2002, ISBN 91 73732583.

No 745 Johan Åberg: Live Help Systems: An Approach to Intelligent Help for Web Information Systems, 2002, ISBN 91-7373-311-3.

No 746 Rego Granlund: Monitoring Distributed Teamwork Training, 2002, ISBN 91-7373-312-1.

No 757 Henrik André-Jönsson: Indexing Strategies for Time Series Data, 2002, ISBN 917373-346-6.

No 747 Anneli Hagdahl: Development of IT-supported Interorganisational Collaboration - A Case Study in the Swedish Public Sector, 2002, ISBN 91-7373-314-8.

No 749 Sofie Pilemalm: Information Technology for NonProfit Organisations - Extended Participatory Design of an Information System for Trade Union Shop Stew ard s, 2002, ISBN 91-7373-318-0.

No 765 Stefan Holmlid: Adapting users: Towards a theory of use quality, 2002, ISBN 91-7373-397-0.

No 771 Magnus Morin: Multimedia Representations of Distributed Tactical Operations, 2002, ISBN 91-7373-4217.

No 772 Pawel Pietrzak: A Type-Based Framew ork for Locating Errors in Constraint Logic Programs, 2002, ISBN 91-7373-422-5.

No 758 Erik Berglund: Library Communication Among Programmers Worldwide, 2002, ISBN 91-7373-349-0.

No 774 Choong-ho Yi: Modelling Object-Oriented Dynamic Systems Using a Logic-Based Framew ork, 2002, ISBN 91-7373-424-1.

No 779 Mathias Broxvall: A Study in the Computational Complexity of Temporal Reasoning, 2002, ISBN 917373-440-3.

No 793 Asmus Pandikow: A Generic Principle for Enabling Interoperability of Structured and Object-Oriented Analysis and Design Tools, 2002, ISBN 91-7373-479-9.

No 785 Lars Hult: Publika Informationstjänster. En studie av den Internetbaserade encyklopedins bruksegenskaper, 2003, ISBN 91-7373-461-6.

No 800 Lars Taxén: A Framework for the Coordination of Complex Systems' Development, 2003, ISBN 917373-604-X
No 808 Klas Gäre: Tre perspektiv på förväntningar och förändringar i samband med införande av informationssystem, 2003, ISBN 91-7373-618-X.

No 821 Mikael Kindborg: Concurrent Comics programming of social agents by children, 2003 , ISBN 91-7373-651-1.

No 823 Christina Ölvingson: On Development of Information Systems with GIS Functionality in Public Health Informatics: A Requirements Engineering Approach, 2003, ISBN 91-7373-656-2.

No 828 Tobias Ritzau: Memory Efficient Hard Real-Time Garbage Collection, 2003, ISBN 91-7373-666-X.

No 833 Paul Pop: Analysis and Synthesis of Communication-Intensive Heterogeneous Real-Time System s, 2003, ISBN 91-7373-683-X.

No 852 Johan Moe: Observing the Dynamic Behaviour of Large Distributed Systems to Improve Development and Testing - An Empirical Study in Software Engineering, 2003, ISBN 91-7373-779-8.

No 867 Erik Herzog: An Approach to Systems Engineering Tool Data Representation and Exchange, 2004, ISBN 91-7373-929-4.

No 872 Aseel Berglund: Augmenting the Remote Control: Studies in Complex Information Navigation for Digital TV, 2004, ISBN 91-7373-940-5.

No 869 Jo Skåmedal: Telecommuting's Implications on Travel and Travel Patterns, 2004, ISBN 91-7373-935-9.

No 870 Linda Askenäs: The Roles of IT - Studies of Organising when Implementing and Using Enterprise Systems, 2004, ISBN 91-7373-936-7.

No 874 Annika Flycht-Eriksson: Design and Use of Ontologies in Information-Providing Dialogue Systems, 2004, ISBN 91-7373-947-2.

No 873 Peter Bunus: Debugging Techniques for EquationBased Languages, 2004, ISBN 91-7373-941-3.

No 876 Jonas Mellin: Resource-Predictable and Efficien Monitoring of Events, 2004, ISBN 91-7373-956-1.

No 883 Magnus Bång: Computing at the Speed of Paper: Ubiquitous Computing Environments for Healthcare Professionals, 2004, ISBN 91-7373-971-5

No 882 Robert Eklund: Disfluency in Swedish humanhuman and human-machine travel booking dialogues, 2004, ISBN 91-7373-966-9.

No 887 Anders Lindström: English and other Foreign Linguistic Elements in Spoken Swedish. Studies of Productive Processes and their Modelling using Finite-State Tools, 2004, ISBN 91-7373-981-2.

No 889 Zhiping Wang: Capacity-Constrained Production-inventory systems - Modelling and Analysis in both a traditional and an e-business context, 2004, ISBN 9185295-08-6.

No 893 Pernilla Qvarfordt: Eyes on Multimodal Interaction, 2004, ISBN 91-85295-30-2.

No 910 Magnus Kald: In the Borderland between Strategy and Management Control - Theoretical Framework and Empirical Evidence, 2004, ISBN 91-85295-82-5

No 918 Jonas Lundberg: Shaping Electronic News: Genre Perspectives on Interaction Design, 2004, ISBN 9185297-14-3.

No 900 Mattias Arvola: Shades of use: The dynamics of interaction design for sociable use, 2004, ISBN 9185295-42-6.

No 920 Luis Alejandro Cortés: Verification and Scheduling Techniques for Real-Time Embedded Systems, 2004, ISBN 91-85297-21-6.

No 929 Diana Szentivanyi: Performance Studies of FaultTolerant Midd lew are, 2005, ISBN 91-85297-58-5. 
No 933 Mikael Cäker: Management Accounting as Constructing and Opposing Customer Focus: Three Case Studies on Management Accounting and Customer Relations, 2005, ISBN 91-85297-64-X.

No 937 Jonas Kvarnström: TALplanner and Other Extensions to Temporal Action Logic, 2005, ISBN 9185297-75-5.

No 938 Bourhane Kadmiry: Fuzzy Gain-Scheduled Visual Servoing for Unmanned Helicopter, 2005, ISBN 9185297-76-3.

No 945 Gert Jervan: Hybrid Built-In Self-Test and Test Generation Techniques for Digital Systems, 2005, ISBN : 91-85297-97-6.

No 946 Anders Arpteg: Intelligent Semi-Structured Information Extraction, 2005, ISBN 91-85297-98-4.

No 947 Ola Angelsmark: Constructing Algorithms for Constraint Satisfaction and Related Problems - Methods and Applications, 2005, ISBN 91-85297-99-2.

No 963 Calin Curescu: Utility-based Optimisation of Resource Allocation for Wireless Networks, 2005 ISBN 91-85457-07-8.

No 972 Björn Johansson: Joint Control in Dynamic Situations, 2005, ISBN 91-85457-31-0.

No 974 Dan Lawesson: An Approach to Diagnosability Analysis for Interacting Finite State Systems, 2005, ISBN 91-85457-39-6.

No 979 Claudiu Duma: Security and Trust Mechanisms for Groups in Distributed Services, 2005, ISBN 91-8545754-X.

No 983 Sorin Manolache: Analysis and Optimisation of Real-Time Systems with Stochastic Behaviour, 2005, ISBN 91-85457-60-4.

No 986 Yuxiao Zhao: Standards-Based Application Integration for Business-to-Business Communications, 2005, ISBN 91-85457-66-3.

No 1004 Patrik Haslum: Admissible Heuristics for Automated Planning, 2006, ISBN 91-85497-28-2.

No 1005 Aleksandra Tešanovic: Developing Reusable and Reconfigurable Real-Time Software using Aspects and Components, 2006, ISBN 91-85497-29-0.

No 1008 David Dinka: Role, Identity and Work: Extending the design and development agenda, 2006, ISBN 91$85497-42-8$.

No 1009 Iakov Nakhimovski: Contributions to the Modeling and Simulation of Mechanical Systems with Detailed Contact Analysis, 2006, ISBN 91-85497-43-X.

No 1013 Wilhelm Dahllöf: Exact Algorithms for Exact Satisfiability Problems, 2006, ISBN 91-85523-97-6.

No 1016 Levon Saldamli: PDEModelica - A High-Level Language for Modeling with Partial Differential Equations, 2006, ISBN 91-85523-84-4

No 1017 Daniel Karlsson: Verification of Component-based Embedded System Designs, 2006, ISBN 91-85523-79-8

No 1018 Ioan Chisalita: Communication and Networking Techniques for Traffic Safety Systems, 2006, ISBN 9185523-77-1

No 1019 Tarja Susi: The Puzzle of Social Activity - The Significance of Tools in Cognition and Cooperation, 2006, ISBN 91-85523-71-2.

No 1021 Andrzej Bednarski: Integrated Optimal Code Generation for Digital Signal Processors, 2006, ISBN 9185523-69-0.

No 1022 Peter Aronsson: Automatic Parallelization of Equation-Based Simulation Programs, 2006, ISBN 9185523-68-2.
No 1030 Robert Nilsson: A Mutation-based Framework for Automated Testing of Timeliness, 2006, ISBN 9185523-35-6.

No 1034 Jon Edvardsson: Techniques for Automatic Generation of Tests from Programs and Specifications, 2006, ISBN 91-85523-31-3.

No 1035 Vaida Jakoniene: Integration of Biological Data, 2006, ISBN 91-85523-28-3.

No 1045 Genevieve Gorrell: Generalized Hebbian Algorithms for Dimensionality Reduction in Natural Language Processing, 2006, ISBN 91-85643-88-2.

No 1051 Yu-Hsing Huang: Having a New Pair of Glasses Applying Systemic Accident Models on Road Safety, 2006, ISBN 91-85643-64-5.

No 1054 Åsa Hedenskog: Perceive those things which cannot be seen - A Cognitive Systems Engineering perspective on requirements management, 2006, ISBN 91-85643-57-2.

No 1061 Cécile Åberg: An Evaluation Platform for Semantic Web Technology, 2007, ISBN 91-85643-31-9.

No 1073 Mats Grindal: Handling Combinatorial Explosion in Software Testing, 2007, ISBN 978-91-85715-74-9.

No 1075 Almut Herzog: Usable Security Policies for Runtime Environments, 2007, ISBN 978-91-85715-65-7.

No 1079 Magnus Wahlström: Algorithms, measures, and upper bounds for Satisfiability and related problems, 2007, ISBN 978-91-85715-55-8.

No 1083 Jesper Andersson: Dynamic Software Architectures, 2007, ISBN 978-91-85715-46-6.

No 1086 Ulf Johansson: Obtaining Accurate and Comprehensible Data Mining Models - An Evolutionary Approach, 2007, ISBN 978-91-85715-34-3.

No 1089 Traian Pop: Analysis and Optimisation of Distributed Embedded Systems with Heterogeneous Scheduling Policies, 2007, ISBN 978-91-85715-27-5.

No 1091 Gustav Nordh: Complexity Dichotomies for CSPrelated Problems, 2007, ISBN 978-91-85715-20-6.

No 1106 Per Ola Kristensson: Discrete and Continuous Shape Writing for Text Entry and Control, 2007, ISBN 97891-85831-77-7.

No 1110 He Tan: Aligning Biomedical Ontologies, 2007, ISBN 978-91-85831-56-2.

No 1112 Jessica Lindblom: Minding the body - Interacting socially through embodied action, 2007, ISBN 978-9185831-48-7.

No 1113 Pontus Wärnestål: Dialogue Behavior Managemen in Conversational Recommender Systems, 2007, ISBN 978-91-85831-47-0.

No 1120 Thomas Gustafsson: Management of Real-Time Data Consistency and Transient Overloads in Embedded Systems, 2007, ISBN 978-91-85831-33-3.

No 1127 Alexandru Andrei: Energy Efficient and Predictable Design of Real-time Embedded Systems, 2007, ISBN 978-91-85831-06-7.

No 1139 Per Wikberg: Eliciting Knowledge from Experts in Modeling of Complex Systems: Managing Variation and Interactions, 2007, ISBN 978-91-85895-66-3.

No 1143 Mehdi Amirijoo: QoS Control of Real-Time Data Services under Uncertain Workload, 2007, ISBN 97891-85895-49-6.

No 1150 Sanny Syberfeldt: Optimistic Replication with Forward Conflict Resolution in Distributed Real-Time Databases, 2007, ISBN 978-91-85895-27-4.

No 1155 Beatrice Alenljung: Envisioning a Future Decision Support System for Requirements Engineering - A Holistic and Human-centred Perspective, 2008, ISBN 978-91-85895-11-3. 
No 1156 Artur Wilk: Types for XML with Application to Xcerpt, 2008, ISBN 978-91-85895-08-3.

No 1183 Adrian Pop: Integrated Model-Driven Development Environments for Equation-Based Object-Oriented Languages, 2008, ISBN 978-91-7393-895-2.

No 1185 Jörgen Skågeby: Gifting Technologies Ethnographic Studies of End-users and Social Media Sharing, 2008, ISBN 978-91-7393-892-1.

No 1187 Imad-Eldin Ali Abugessaisa: Analytical tools and information-sharing methods supporting road safety organizations, 2008, ISBN 978-91-7393-887-7.

No 1204 H. Joe Steinhauer: A Representation Scheme for Description and Reconstruction of Object Configurations Based on Qualitative Relations, 2008, ISBN 978-91-7393-823-5.

No 1222 Anders Larsson: Test Optimization for Core-based System-on-Chip, 2008, ISBN 978-91-7393-768-9.

No 1238 Andreas Borg: Processes and Models for Capacity Requirements in Telecommunication Systems, 2009. ISBN 978-91-7393-700-9.

No 1240 Fredrik Heintz: DyKnow: A Stream-Based Knowledge Processing Middleware Framework, 2009, ISBN 978-91-7393-696-5.

No 1241 Birgitta Lindström: Testability of Dynamic RealTime Systems, 2009, ISBN 978-91-7393-695-8.

No 1244 Eva Blomqvist: Semi-automatic Ontology Construction based on Patterns, 2009, ISBN 978-91-7393-683-5.

No 1249 Rogier Woltjer: Functional Modeling of Constraint Management in Aviation Safety and Command and Control, 2009, ISBN 978-91-7393-659-0.

No 1260 Gianpaolo Conte: Vision-Based Localization and Guidance for Unmanned Aerial Vehicles, 2009, ISBN 978-91-7393-603-3.

No 1262 AnnMarie Ericsson: Enabling Tool Support for Formal Analysis of ECA Rules, 2009, ISBN 978-91-7393598-2.

No 1266 Jiri Trnka: Exploring Tactical Command and Control: A Role-Playing Simulation Approach, 2009 , ISBN 978-91-7393-571-5.

No 1268 Bahlol Rahimi: Supporting Collaborative Work through ICT - How End-users Think of and Adopt Integrated Health Information Systems, 2009, ISBN 978-91-7393-550-0.

No 1274 Fredrik Kuivinen: Algorithms and Hardness Results for Some Valued CSPs, 2009, ISBN 978-91-7393-525-8.

No 1281 Gunnar Mathiason: Virtual Full Replication for Scalable Distributed Real-Time Databases, 2009 ISBN 978-91-7393-503-6.

No 1290 Viacheslav Izosimov: Scheduling and Optimization of Fault-Tolerant Distributed Embedded Systems, 2009, ISBN 978-91-7393-482-4.

No 1294 Johan Thapper: Aspects of a Constraint Optimisation Problem, 2010, ISBN 978-91-7393-464-0.

No 1306 Susanna Nilsson: Augmentation in the Wild: User Centered Development and Evaluation of Augmented Reality Applications, 2010, ISBN 978-91 7393-416-9.

No 1313 Christer Thörn: On the Quality of Feature Models, 2010, ISBN 978-91-7393-394-0.

No 1321 Zhiyuan He: Temperature Aware and DefectProbability Driven Test Scheduling for System-onChip, 2010, ISBN 978-91-7393-378-0.

No 1333 David Broman: Meta-Languages and Semantics for Equation-Based Modeling and Simulation, 2010, ISBN 978-91-7393-335-3.

No 1337 Alexander Siemers: Contributions to Modelling and Visualisation of Multibody Systems Simulations with
Detailed Contact Analysis, 2010, ISBN 978-91-7393317-9.

No 1354 Mikael Asplund: Disconnected Discoveries: Availability Studies in Partitioned Networks, 2010, ISBN 978-91-7393-278-3.

No 1359 Jana Rambusch: Mind Games Extended Understanding Gameplay as Situated Activity, 2010, ISBN 978-91-7393-252-3.

No 1373 Sonia Sangari: Head Movement Correlates to Focus Assignment in Swedish,2011,ISBN 978-91-7393-154-0.

No 1374 Jan-Erik Källhammer: Using False Alarms when Developing Automotive Active Safety Systems, 2011, ISBN 978-91-7393-153-3.

No 1375 Mattias Eriksson: Integrated Code Generation, 2011, ISBN 978-91-7393-147-2

No 1381 Ola Leifler: Affordances and Constraints of Intelligent Decision Support for Military Command and Control - Three Case Studies of Support Systems, 2011, ISBN 978-91-7393-133-5.

No 1386 Soheil Samii: Quality-Driven Synthesis and Optimization of Embedded Control Systems, 2011, ISBN 978-91-7393-102-1.

No 1419 Erik Kuiper: Geographic Routing in Intermittentlyconnected Mobile Ad Hoc Networks: Algorithms and Performance Models, 2012, ISBN 978-91-7519981-8.

No 1451 Sara Stymne: Text Harmonization Strategies for Phrase-Based Statistical Machine Translation, 2012 ISBN 978-91-7519-887-3.

No 1455 Alberto Montebelli: Modeling the Role of Energy Management in Embodied Cognition, 2012, ISBN 978-91-7519-882-8.

No 1465 Mohammad Saifullah: Biologically-Based Interactive Neural Network Models for Visual Attention and Object Recognition, 2012, ISBN 978-91-7519-838-5.

No 1490 Tomas Bengtsson: Testing and Logic Optimization Techniques for Systems on Chip, 2012, ISBN 978-917519-742-5.

No 1481 David Byers: Improving Software Security by Preventing Known Vulnerabilities, 2012, ISBN 97891-7519-784-5

No 1496 Tommy Färnqvist: Exploiting Structure in CSP related Problems, 2013, ISBN 978-91-7519-711-1.

No 1503 John Wilander: Contributions to Specification Implementation, and Execution of Secure Software, 2013, ISBN 978-91-7519-681-7.

No 1506 Magnus Ingmarsson: Creating and Enabling the Useful Service Discovery Experience, 2013, ISBN 978 91-7519-662-6.

No 1547 Wladimir Schamai: Model-Based Verification of Dynamic System Behavior against Requirements: Method, Language, and Tool, 2013, ISBN 978-917519-505-6.

No 1551 Henrik Svensson: Simulations, 2013, ISBN 978-917519-491-2

No 1559 Sergiu Rafiliu: Stability of Adaptive Distributed Real-Time Systems with Dynamic Resource Management, 2013, ISBN 978-91-7519-471-4.

No 1581 Usman Dastgeer: Performance-aware Componen Composition for GPU-based Systems, 2014, ISBN 978-91-7519-383-0.

No 1602 Cai Li: Reinforcement Learning of Locomotion based on Central Pattern Generators, 2014, ISBN 978-917519-313-7.

No 1652 Roland Samlaus: An Integrated Developmen Environment with Enhanced Domain-Specific 
Interactive Model Validation, 2015, ISBN 978-917519-090-7.

No 1663 Hannes Uppman: On Some Combinatorial Optimization Problems: Algorithms and Complexity, 2015, ISBN 978-91-7519-072-3.

No 1664 Martin Sjölund: Tools and Methods for Analysis, Debugging, and Performance Improvement of Equation-Based Models, 2015, ISBN 978-91-7519-071-6.

No 1666 Kristian Stavåker: Contributions to Simulation of Modelica Models on Data-Parallel Multi-Core Architectures, 2015, ISBN 978-91-7519-068-6.

No 1680 Adrian Lifa: Hardware/ Software Codesign of Embedded Systems with Reconfigurable and Heterogeneous Platforms, 2015, ISBN 978-91-7519-040 2.

No 1685 Bogdan Tanasa: Timing Analysis of Distributed Embedded Systems with Stochastic Workload and Reliability Constraints, 2015, ISBN 978-91-7519-022-8.

No 1691 Håkan Warnquist: Troubleshooting Trucks Automated Planning and Diagnosis, 2015, ISBN 978 91-7685-993-3

No 1702 Nima Aghaee: Thermal Issues in Testing of Advanced Systems on Chip, 2015, ISBN 978-91-7685949-0.

No 1715 Maria Vasilevskaya: Security in Embedded Systems: A Model-Based Approach with Risk Metrics, 2015 ISBN 978-91-7685-917-9.

No 1729 Ke Jiang: Security-Driven Design of Real-Time Embedded System, 2016, ISBN 978-91-7685-884-4.

No 1733 Victor Lagerkvist: Strong Partial Clones and the Complexity of Constraint Satisfaction Problems: Limitations and Applications, 2016, ISBN 978-91-7685856-1

No 1734 Chandan Roy: An Informed System Development Approach to Tropical Cyclone Track and Intensity Forecasting, 2016, ISBN 978-91-7685-854-7.

No 1746 Amir Aminifar: Analysis, Design, and Optimization of Embedded Control Systems, 2016, ISBN 978-917685-826-4.

No 1747 Ekhiotz Vergara: Energy Modelling and Fairness for Efficient Mobile Communication, 2016, ISBN 978-91-7685-822-6.

No 1748 Dag Sonntag: Chain Graphs - Interpretations, Expressiveness and Learning Algorithms, 2016, ISBN 978-91-7685-818-9.

No 1768 Anna Vapen: Web Authentication using ThirdParties in Untrusted Environments, 2016, ISBN 978-91-7685-753-3

No 1778 Magnus Jandinger: On a Need to Know Basis: A Conceptual and Methodological Framew ork for Modelling and Analysis of Information Demand in an Enterprise Context, 2016, ISBN 978-91-7685-713-7.

No 1798 Rahul Hiran: Collaborative Network Security: Targeting Wide-area Routing and Edgenetw ork Attacks, 2016, ISBN 978-91-7685-662-8.

No 1813 Nicolas Melot: Algorithms and Framework for Energy Efficient Parallel Stream Computing on Many-Core Architectures, 2016, ISBN 978-91-7685623-9.

No 1823 Amy Rankin: Making Sense of Adaptations: Resilience in High-Risk Work, 2017, ISBN 978-917685-596-6.
No 1831 Lisa Malmberg: Building Design Capability in the Public Sector: Expanding the Horizons of Development, 2017, ISBN 978-91-7685-585-0.

No 1851 Marcus Bendtsen: Gated Bayesian Networks, 2017, ISBN 978-91-7685-525-6.

No 1854 Meysam Aghighi: Computational Complexity of some Optimization Problems in Planning, 2017, ISBN 978-91-7685-519-5.

Linköping Studies in Arts and Science

No 504 Ing-Marie Jonsson: Social and Emotional Characteristics of Speech-based In-Vehicle Information Systems: Impact on Attitude and Driving Behaviour, 2009, ISBN 978-91-7393-478-7.

No 586 Fabian Segelström: Stakeholder Engagement for Service Design: How service designers identify and communicate insights, 2013, ISBN 978-91-7519-554-4.

No 618 Johan Blomkvist: Representing Future Situations of Service: Prototyping in Service Design, 2014, ISBN 978-91-7519-343-4.

No 620 Marcus Mast: Human-Robot Interaction for SemiAutonomous Assistive Robots, 2014, ISBN 978-917519-319-9.

No 677 Peter Berggren: Assessing Shared Strategic Understanding, 2016, ISBN 978-91-7685-786-1.

No 695 Mattias Forsblad: Distributed cognition in home environments: The prospective memory and cognitive practices of older adults, 2016, ISBN 97891-7685-686-4.

\section{Linköping Studies in Statistics}

No 9 Davood Shahsavani: Computer Experiments Designed to Explore and Approximate Complex Deterministic Models, 2008, ISBN 978-91-7393-976-8.

No 10 Karl Wahlin: Roadmap for Trend Detection and Assessment of Data Quality, 2008, ISBN 978-91-7393792-4.

No 11 Oleg Sysoev: Monotonic regression for large multivariate datasets, 2010, ISBN 978-91-7393-412-1.

No 13 Agné Burauskaite-Harju: Characterizing Temporal Change and Inter-Site Correlations in Daily and Subdaily Precipitation Extremes, 2011, ISBN 978-91-7393110-6.

\section{Linköping Studies in Information Science}

No 1 Karin Axelsson: Metodisk systemstrukturering- at skapa samstämmighet mellan informationssystemarkitektur och verksamhet, 1998. ISBN-9172-19-296-8.

No 2 Stefan Cronholm: Metodverktyg och användbarhet en studie av datorstödd metodbaserad systemutveckling, 1998, ISBN-9172-19-299-2.

No 3 Anders Avdic: Användare och utvecklare - om anveckling med kalkylprogram, 1999. ISBN-91-7219606-8.

No 4 Owen Eriksson: Kommunikationskvalitet hos informationssystem och affärsprocesser, 2000, ISBN 917219-811-7.

No 5 Mikael Lind: Från system till process - kriterier för processbestämning vid verksamhetsanalys, 2001, ISBN 91-7373-067-X.

No 6 Ulf Melin: Koordination och informationssystem i företag och nätverk, 2002, ISBN 91-7373-278-8.

No 7 Pär J. Ågerfalk: Information Systems Actability - Understanding Information Technology as a Tool for Business Action and Communication, 2003, ISBN 917373-628-7. 
No 8 Ulf Seigerroth: Att förstå och förändra systemutvecklingsverksamheter - en taxonomi för metautveckling, 2003, ISBN91-7373-736-4.

No 9 Karin Hedström: Spår av datoriseringens värden Effekter av IT i äldreomsorg, 2004, ISBN 91-7373-9634.

No 10 Ewa Braf: Knowledge Demanded for Action Studies on Knowledge Mediation in Organisations, 2004, ISBN 91-85295-47-7.

No 11 Fredrik Karlsson: Method Configuration method and computerized tool support, 2005, ISBN 91-8529748-8.

No 12 Malin Nordström: Styrbar systemförvaltning - Att organisera systemförvaltningsverksamhet med hjälp av effektiva förvaltningsobjekt, 2005, ISBN 91-8529760-7.

No 13 Stefan Holgersson: Yrke: POLIS - Yrkeskunskap, motivation, IT-system och andra förutsättningar för polisarbete, 2005, ISBN 91-85299-43-X.

No 14 Benneth Christiansson, Marie-Therese Christiansson: Mötet mellan process och komponent - mot ett ramverk för en verksamhetsnära kravspecifikation vid anskaffning av komponentbaserade informationssystem, 2006, ISBN 91-8564322-X. 